\title{
NATURAL ZEOLITE REMOVAL CAPACITY OF HEAVY METALLIC IONS
}

\author{
by \\ Amanda Lidia Alaica-Ciosek \\ B.Eng. (Hons.) (2010), M.A.Sc. (2012) \\ Ryerson University

\begin{abstract}
A Dissertation
presented to Ryerson University

in partial fulfillment of the

requirements for the degree of

Doctor of Philosophy

in the Program of

Civil Engineering
\end{abstract}

Toronto, Ontario, Canada, 2018

(C) Amanda Lidia Alaica-Ciosek, 2018 


\section{AUTHOR'S DECLARATION FOR ELECTRONIC SUBMISSION OF A DISSERTATION}

I hereby declare that I am the sole author of this Dissertation. This is a true copy of the Dissertation, including any required final revisions, as accepted by my examiners.

I authorize Ryerson University to lend this Dissertation to other institutions or individuals for the purpose of scholarly research.

I further authorize Ryerson University to reproduce this Dissertation by photocopying or by other means, in total or in part, at the request of other institutions or individuals for the purpose of scholarly research.

I understand that my Dissertation may be made electronically available to the public. 


\title{
NATURAL ZEOLITE REMOVAL CAPACITY OF HEAVY METALLIC IONS
}

\author{
Amanda Lidia Alaica-Ciosek \\ Doctor of Philosophy \\ Civil Engineering, Ryerson University, 2018
}

\begin{abstract}
Our ecosystem is at risk by many anthropogenic activities, which include the release of industrial wastewater effluents laden with toxic heavy metals. There is a long history and a continued demand for proper evaluation and predication of water quality and management, in order to protect surrounding water resources and all living species. Undeniably, these pollutants (heavy metallic ions; HMIs) are a detrimental threat, and must be removed by advanced treatment technology prior to discharge. One such strategy would be by the process of sorption (adsorption/ion-exchange), which has advanced among researchers. Zeolites in particular have attracted researchers' interests, being a naturally abundant, cost-effective mineral, with high cation exchange capacity and selectivity of certain metals. They are considered as a strong candidate for the removal of HMIs, and hold the potential for regeneration, recovery and reuse in pertinent industrial applications.

This study investigates the sorption process by natural zeolite (clinoptilolite) of HMIs that are commonly found in industrial wastewater effluent, namely lead $\left(\mathrm{Pb}^{2+}\right)$, copper $\left(\mathrm{Cu}^{2+}\right)$, iron $\left(\mathrm{Fe}^{3+}\right)$, nickel $\left(\mathrm{Ni}^{2+}\right)$ and zinc $\left(\mathrm{Zn}^{2+}\right)$. The HMIs are combined in acidic, synthetic simple-solute solutions of various (single-, dual-, triple-, multi-) component systems, in a controlled environment for improved quantification and identification of the important trends; in order to address existing limitations in multi-component system research. The analytical methodology of ICP-AES was employed for all quantitative detection and analyses.

The project consists of four phases in the analysis of: (1) the effects of preliminary parameters and operative conditions (particle size, sorbent-to-sorbate dosage, influent concentration, contact time, set-temperature, and heat pre-treatment), (2) HMIs component system combinations and selectivity order, (3) kinetic modelling trends, and (4) the design of a packed, fixed-bed, dual-column sorption treatment system prototype.
\end{abstract}


Under the testing conditions, this study demonstrates a strong correlation with the pseudosecond-order kinetic model in batch-mode analysis, as well as a relationship among the empty bed contact time, breakthrough capacity, and usage rate in continuous-mode investigations. A key sorption trend among the HMIs selected is well-established in all four phases as $\mathrm{Pb}^{2+}>>\mathrm{Fe}^{3+}>\mathrm{Cu}^{2+}>\mathrm{Zn}^{2+}>>\mathrm{Ni}^{2+}$; providing significant validation of this experimental design. The system prototype is a platform for the advancement of intelligent process controls. It is envisaged that this research will provide essential information to the industrial wastewater treatment industry for the design and implementation of innovative zeolite-based sorption technology.

Keywords: Natural Zeolite; Clinoptilolite; Heavy Metallic Ions; Sorption Capacity; Adsorption; Ion-Exchange; Removal Efficiency; Operation Parameters; Selectivity; Kinetic Modelling; Packed Fixed-Bed Columns; ICP-AES; Automated Design; Intelligent Process Controls Platform. 


\section{ACKNOWLEDGEMENTS}

First and foremost, I would like to express my deepest gratitude to my direct supervisor Dr. Grace K. Luk. I sincerely appreciate all of her care and guidance throughout my academic career; it was an honour to be her student.

I would like to thank my supervisory committee members of the Department of Civil Engineering at Ryerson University; to Dr. James Li, my co-supervisor who provided significant support to the completion of my degree, for which I am truly grateful; to Dr. Ahmed Shaker and Dr. Darko Joksimovic for their thoughtful insight. Thank you to Dr. Lynda H. McCarthy of the Department of Chemistry and Biology at Ryerson University for her expertise and significant assessment of my research. Finally, thank you to Dr. Ronald L. Droste of the Department of Civil Engineering at the University of Ottawa, for his time and consideration, expertise and critical review of my research as the external examiner. I would also like to extend my gratitude to the numerous anonymous reviewers who provided helpful suggestions to improve the manuscripts presented in the Dissertation document.

Thank you to Ms. Valerie Phelan (Radiation/Chemical/Biosafety Officer) and Mr. Eric Ambroise (Lab Safety Officer) of the Ryerson Integrated Risk Management (IRM) for all their assistance as I established the safety and waste management protocol of the research project. Thank you to Mr. Dan Mathers, the supervisor of the Analytical Lab for Environmental Science Research and Training (ANALEST) at the Department of Chemistry of the University of Toronto, for ad-hoc training of the analytical technology and kind support throughout my experimental analyses.

Finally, I would like to express my heartfelt appreciation to Mr. Robin Luong, the Environmental Technician of the Department of Civil Engineering at Ryerson University; who was beyond technical support throughout my academic career. He has taught me the fundamentals of quality research skills and the importance of perseverance, which I will take with me in my professional career and in life.

To my family, both near and far, thank you for all of your love throughout this incredible journey; I love you and cherish you always. 


\section{DEDICATION}

TO MY PARENTS - LIDIJA + ZELJKO ALAICA.

Ја вас волим.

“THE ONLY WAY OUT IS THROUGH.” - R. FROST. 


\section{PREFACE}

This Dissertation is submitted for the degree of Doctor of Philosophy at Ryerson University. The research described herein was conducted under the direct supervision of Professor Dr. Grace K. Luk of the Department of Civil Engineering, Ryerson University, between September 2012 and August 2017.

This Dissertation is based on a four-phase research project, and is submitted as a manuscript-style document, composed of eight chapters and six appendices. Chapter 1 is the introduction and critical review of the experimental design of the research topic. Chapter 2 defines the study objectives and scope, document structure as well as the statement of authorship. Chapter 3 presents the analytical procedures and quality control measures implemented; which are consolidated for cohesion in the document. Chapters 4, 5, 6, and 7 are the manuscripts that discuss the significant findings of each phase of the research project. Chapter 8 provides the major conclusions and recommendations for future implementation of the technology in engineering practice. The appendices provide the details of all the experimental procedures and schedule (A), analytical technology operating procedure (B), as well as technical conference papers $(\mathrm{C}, \mathrm{D}, \mathrm{E})$. Appendix $\mathrm{F}$ highlights a cover featured journal publication that investigates the capacity of natural zeolite to remove nutrients from wastewater; a former research endeavour investigated by the Ph.D. Candidate.

This research is to the best of knowledge original, expect where acknowledgements and references are made to previous work. Neither this, nor any substantially similar Dissertation has been or is being submitted for any other degree, diploma or other qualification at any other university. This Dissertation document contains less than 60,000 words, within 200 pages. 


\section{TABLE OF CONTENTS}

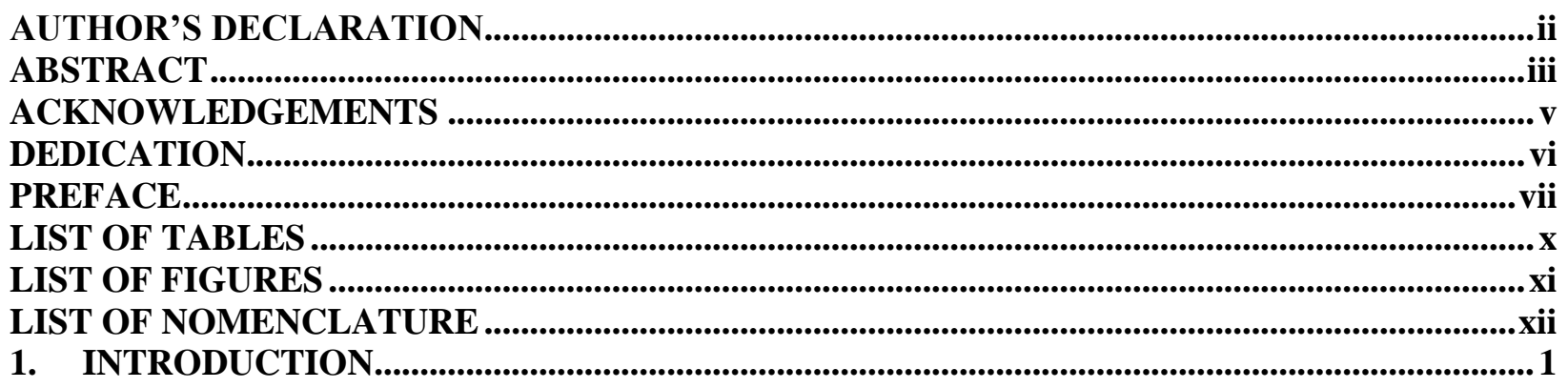

1.1. Industrial Wastewater Effluents -

Characteristics, Impacts, and Treatment Methods ......................................................... 1

1.2. Selection of Sorbent Material - Natural Zeolite Mineral Characteristics ................................... 7

1.2.1. Chemical Composition and Structure ......................................................................

1.2.2. Sorption - Adsorption and Ion-Exchange Capacity …................................................... 12

1.3. Selection of Experimental Influent Stock ........................................................................... 17

1.4. Techniques and Challenges of Current Treatment Technology Research ................................ 22

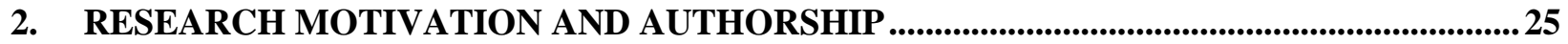

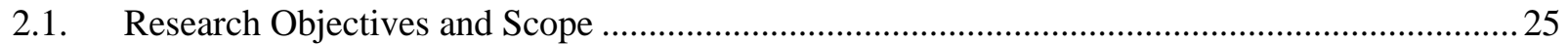

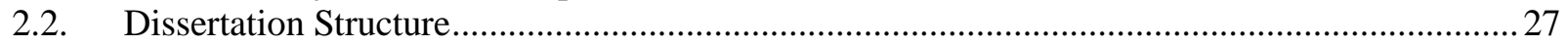

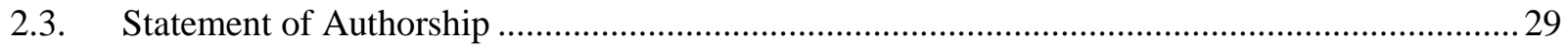

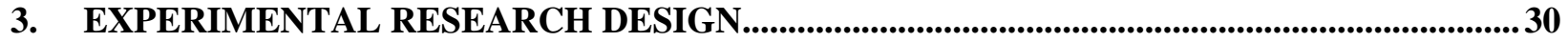

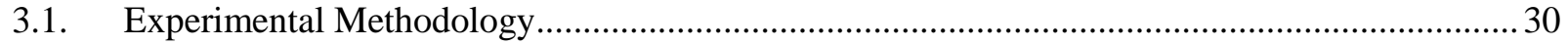

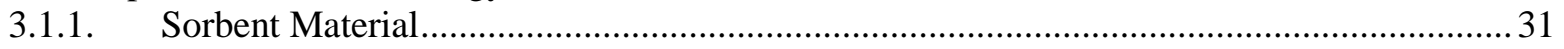

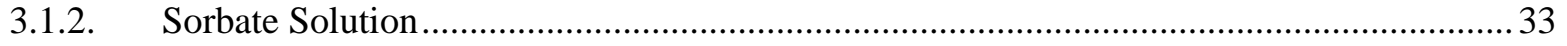

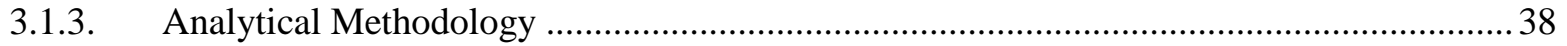

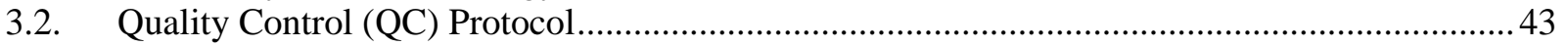

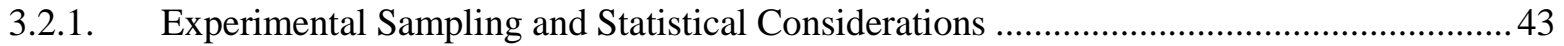

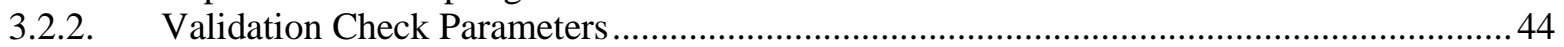

3.2.3. Influent Concentration Detection Trends ....................................................................... 45

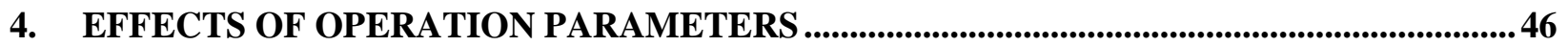

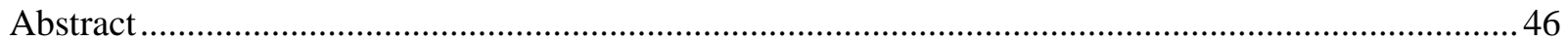

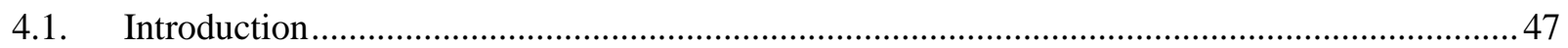

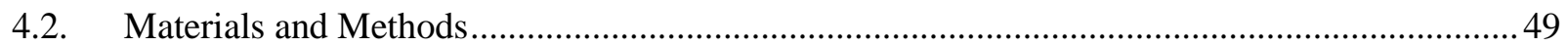

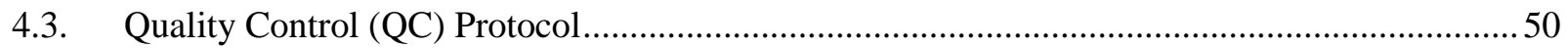

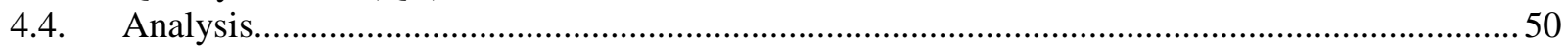

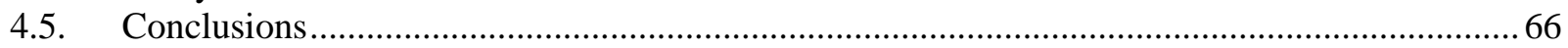

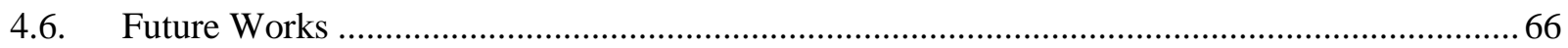

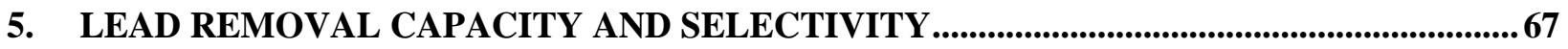

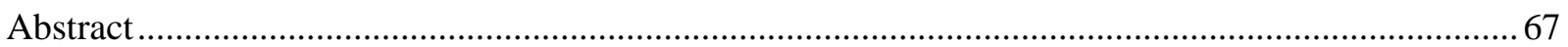

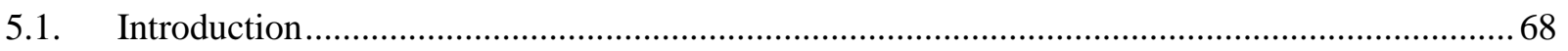

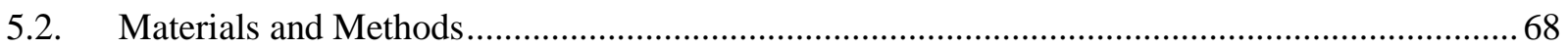

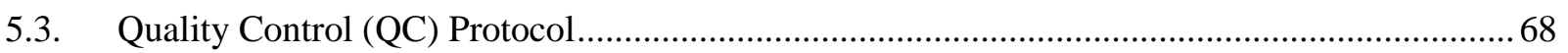

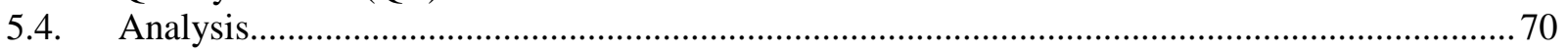

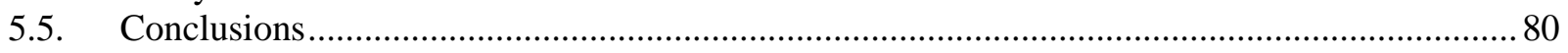

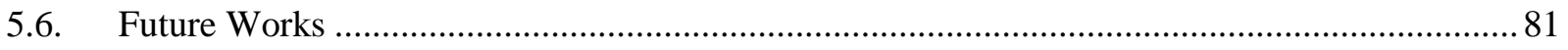




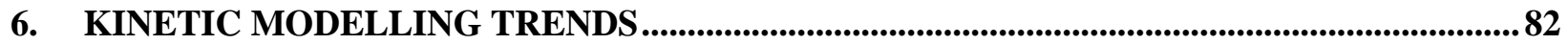

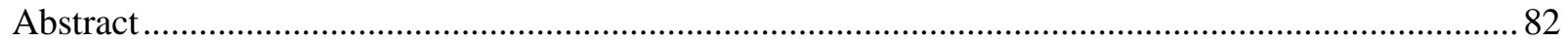

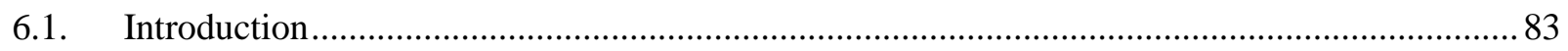

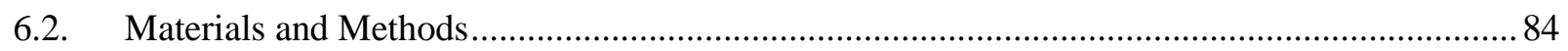

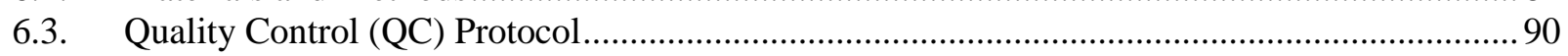

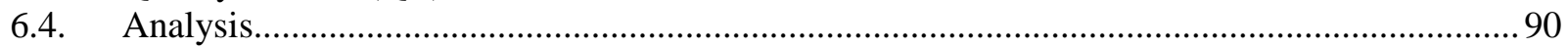

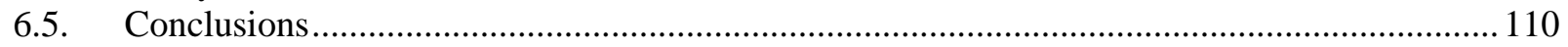

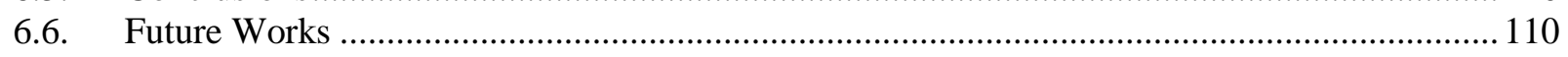

7. DUAL-COLUMN SORPTION SYSTEM PERFORMANCE ..................................................111

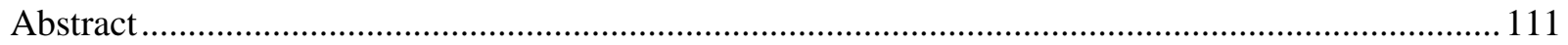

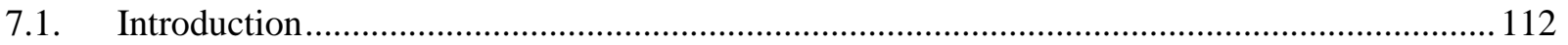

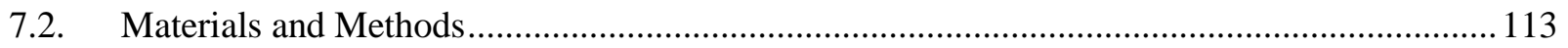

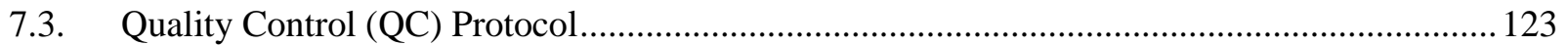

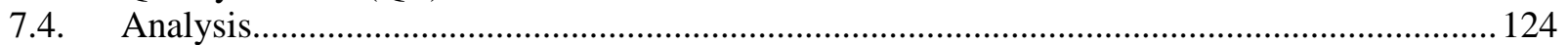

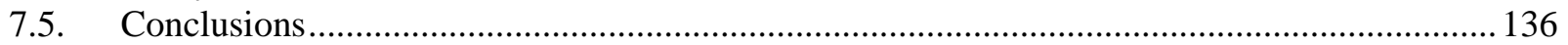

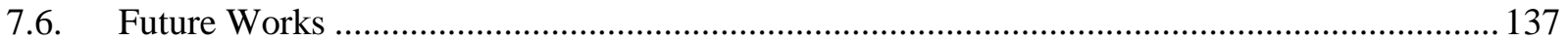

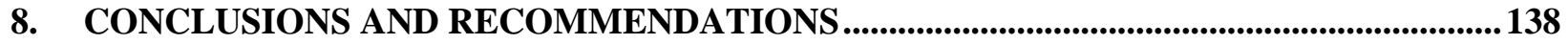

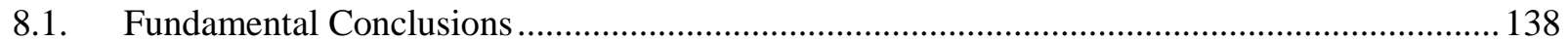

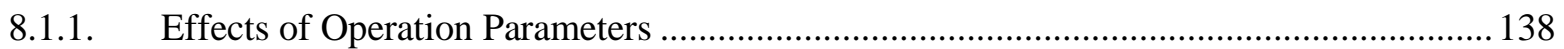

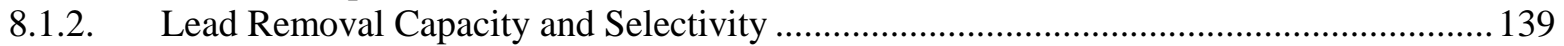

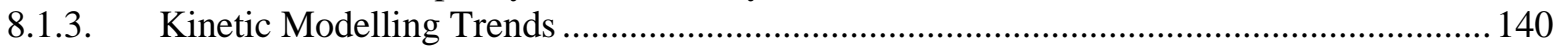

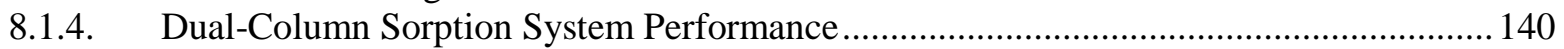

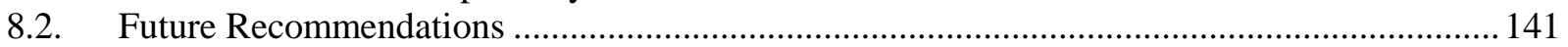

APPENDIX A. Experimental Methodology Procedures .......................................................................... 148

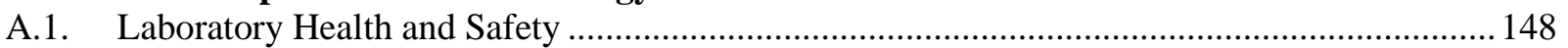

A.2. Materials and Equipment - Inventory and Specifications .............................................. 150

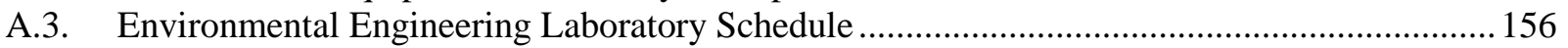

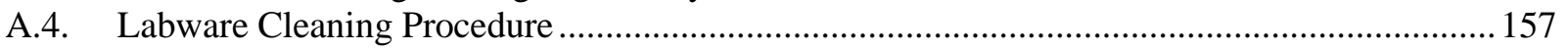

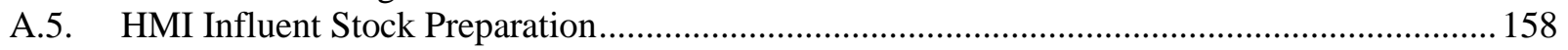

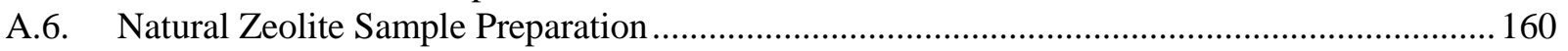

A.7. Hydrothermal Pre-Treatment Preparation .......................................................................... 163

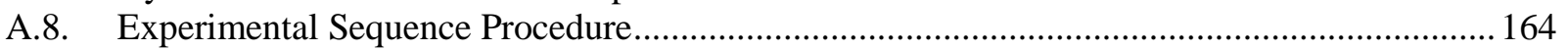

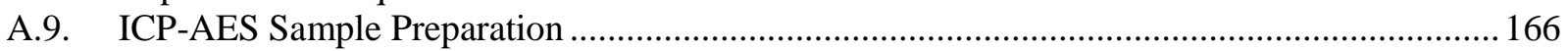

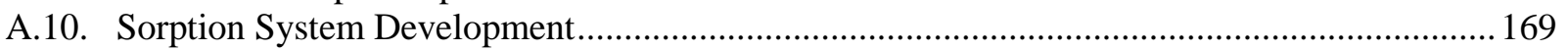

APPENDIX B. ICP-AES Standard Operating Procedure .............................................................................170

APPENDIX C. WEAO2016 Technical Symposium .............................................................................................177

APPENDIX D. WEAO2017 Technical Symposium ..........................................................................178

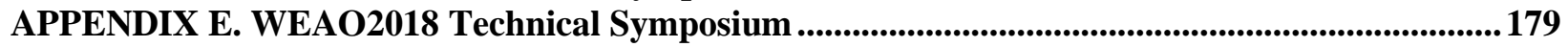

APPENDIX F. Water Environment Research Journal Publication.................................................180

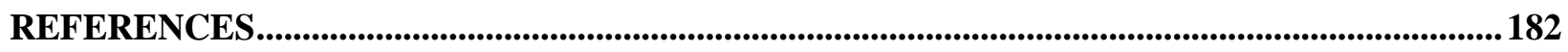




\section{LIST OF TABLES}

Table 1.1 Water Standards - Maximum Contaminant Level of Heavy Metals (mg/L) ..........................5

Table 1.2 Existing Trends in Influent Stock, Zeolite Material and Heavy Metals of Interest................... 18

Table 1.3 Metal Concentrations of Various Industrial Wastewater Effluents (mg/L) ............................2 21

Table 2.1 Dissertation Chapters - Research Project Phases and Focus ..................................................26

Table 3.1 Established Experimental Factors throughout Analysis .............................................................30

Table 3.2 Preliminary Distribution of $+14-40$ Zeolite Supply ............................................................. 32

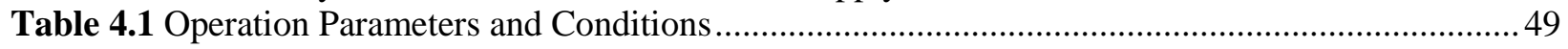

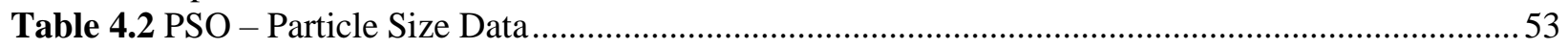

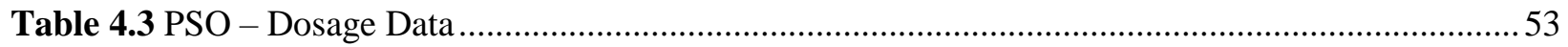

Table 4.4 The HMI Removal Variation by Influent Concentration ...................................................55

Table 4.5 The HMI Removal Variation by Contact Time at $22^{\circ} \mathrm{C}$ Set-Temperature ................................56

Table 4.6 The HMI Removal Variation by Set-Temperature at 180 Contact Minutes .............................57

Table 4.7 HMI Uptake (meq/g) by Heat Pre-Treatment Level .............................................................61

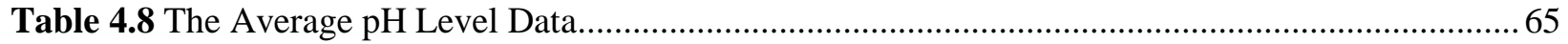

Table 5.1 Calibration Standard 50 QC Check of Various Component Systems ......................................69

Table 5.2 Sample Preparation with $\mathrm{pH}$ Level Verification ................................................................... 71

Table 5.3 ICP-AES HMI Results of Various Component Systems ...................................................... 72

Table 5.4 The HMI Removal (meq/L) in the Various Component Systems Relative to the Lead Ion ......76

Table 5.5 The HMI Uptake in the Various Component Systems by Zeolite........................................... 77

Table 5.6 Selectivity Ratios (SR) of the Various Component Systems Relative to Lead Uptake ............. 77

Table 6.1 ICP-AES HMI Results of the Single-Component Systems....................................................92

Table 6.2 ICP-AES HMI Results of the Dual-Component Systems .....................................................92

Table 6.3 ICP-AES HMI Results of the Triple- and Multi-Component Systems ...................................93

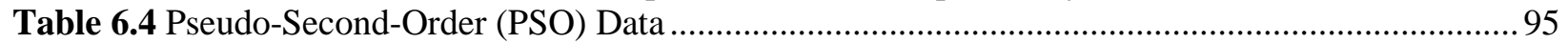

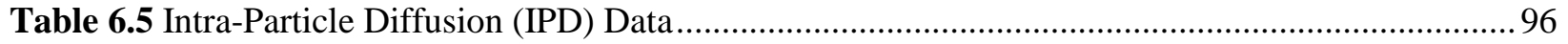

Table 6.6 Pseudo-Second-Order (PSO) and Elovich Data Comparison ................................................98

Table 6.7 Experimental Total and Theoretical Equilibrium HMI Uptake System Observations.............. 103

Table 6.8 The Sorption Trends of Heavy Metallic Ions on Various Sorbent Materials .......................... 104

Table 6.9 Rate Expressions for Reactant A Concentration in a Batch-Mode Reactor System ................ 105

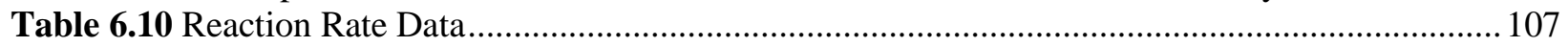

Table 7.1 ICP-AES Generated Multi-Component Stock Concentration............................................... 123

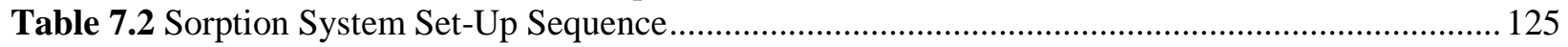

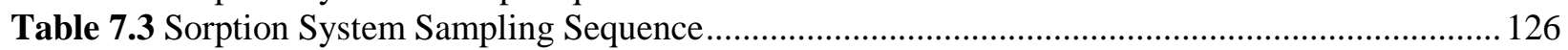

Table 7.4 The pH Levels of Selected Sorption Column Samples ....................................................... 128

Table 7.5 The HMI Concentration (meq/L) and Percent Removal (\%R) in the Sorption Columns ........ 131

Table 7.6 System Breakthrough Point Performance ............................................................................. 134

Table A.1 Inventory of Consumable Materials and Related Equipment............................................ 150

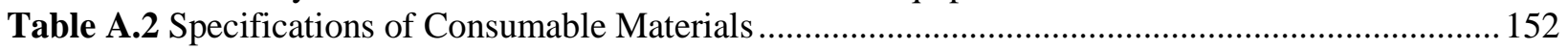

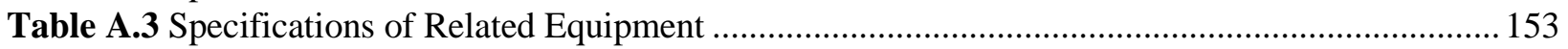

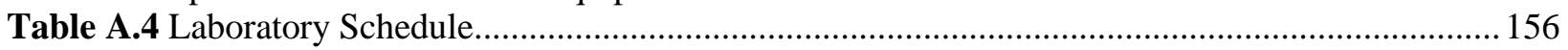

Table A.5 Influent Concentration Computation ................................................................................. 158

Table A.6 Particle Size Breakdown of Natural Zeolite Sorbent Supply ............................................. 161

Table A.7 Orbital Shaker Sample Bottle Contact Sequence ........................................................... 165

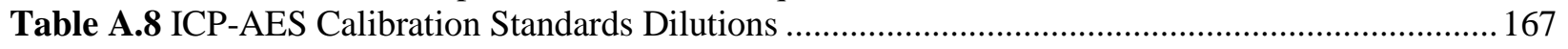

Table A.9 Number of 50\% Dilutions (DIL\#) for each Component System Sample ............................... 167 


\section{LIST OF FIGURES}

Figure 1.1 Relationship of the Clinoptilolite Framework and a Trapped Cation.................................... 10

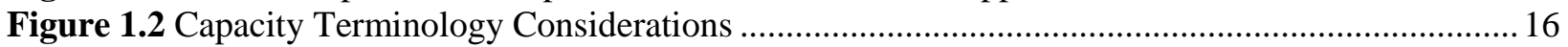

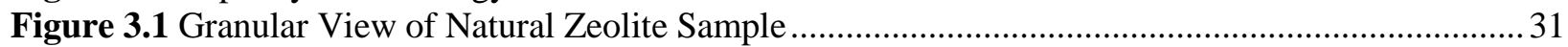

Figure 4.1 HMI Uptake based on Particle Size Parameter...............................................................51

Figure 4.2 HMI Percent Removal based on Dosage Parameter ........................................................51

Figure 4.3 Total HMI Uptake based on Contact Time Parameter ....................................................57

Figure 4.4 Total HMI Uptake based on Set-Temperature Parameter....................................................58

Figure 4.5 Percentage of Non-Heated Level Total HMI Uptake ....................................................62

Figure 4.6 Heat Pre-Treatment Variation of Total HMI Uptake over Time .......................................... 63

Figure 4.7 SEM Images of Natural Zeolite Exposure to Heat-Pre-treatment ...........................................64

Figure 5.1 SEM Images of Natural Zeolite Exposure to Multi-Component Solution Over Time ............. 73

Figure 5.2 EDS Spectrum of Zeolite Sample Exposure to Multi-Component Solution ...........................74

Figure 5.3 Percent Removal of Lead in the Various Component Systems ............................................ 75

Figure 5.4 Percent Removal of HMIs in the Multi-Component System.................................................76

Figure 5.5 Total HMI Uptake in the Various Component Systems over Time........................................ 78

Figure 6.1 Total HMI Percent Removal versus Contact Time..............................................................93

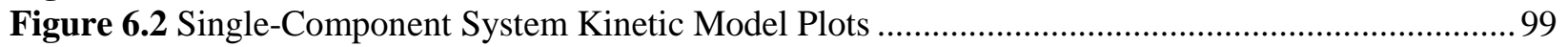

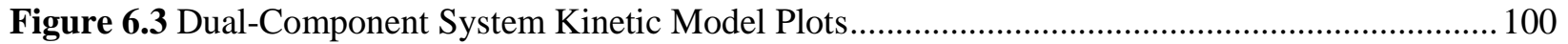

Figure 6.4 Triple- and Multi-Component System Kinetic Model Plots.............................................. 101

Figure 6.5 Multi-component System - Uptake Over Time alongside Kinetic Models........................... 102

Figure 6.6 Single-Component System Reaction Rate Decay Profiles .................................................. 108

Figure 7.1 Schematic of Automated Sorption System Prototype Flow Path Layout .............................. 117

Figure 7.2 Image of Automated Sorption System Prototype Design .................................................... 122

Figure 7.3 The Multi-Component System Breakthrough Curve …....................................................... 132

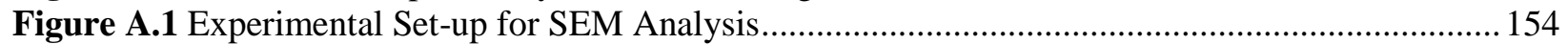

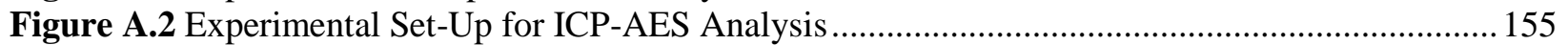

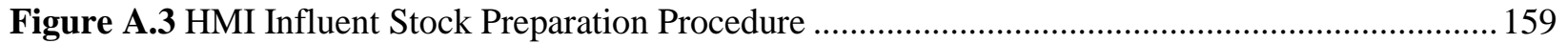

Figure A.4 Natural Zeolite Sample Preparation - Cleaning Cycle Procedure ....................................... 162

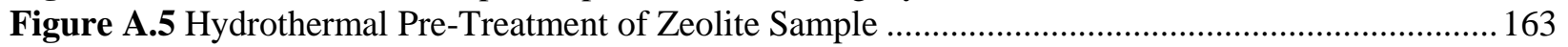

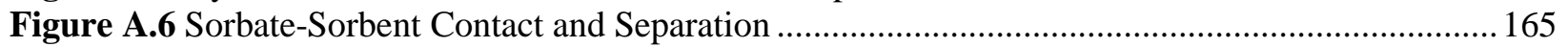

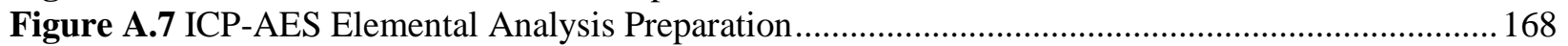

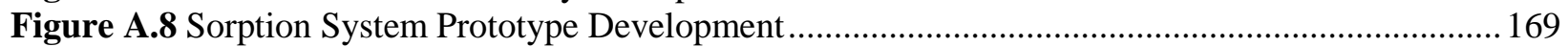

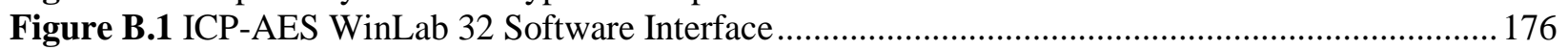

Figure F.1 Water Environment Research Journal Publication........................................................... 181 


\section{LIST OF NOMENCLATURE}

\section{LIST OF ABBREVIATIONS}

\begin{tabular}{|c|c|c|c|}
\hline \multirow{2}{*}{\multicolumn{2}{|c|}{$\begin{array}{l}\text { HMI } \\
\text { ICP-AES }\end{array}$}} & \multicolumn{2}{|c|}{ Heavy Metallic Ion } \\
\hline & & \multicolumn{2}{|c|}{ Inductively Coupled Plasma - Atomic Emission Spectroscopy } \\
\hline \multicolumn{2}{|c|}{ SEM/EDS } & \multicolumn{2}{|c|}{ Scanning Electron Microscopy/Energy Dispersive X-Ray Spectroscopy } \\
\hline \multicolumn{2}{|c|}{ GLWQA } & \multicolumn{2}{|c|}{ Great Lakes Water Quality Agreement } \\
\hline \multicolumn{2}{|c|}{ GAC } & \multicolumn{2}{|c|}{ Granulated Activated Carbon } \\
\hline \multicolumn{2}{|c|}{ Si/Al } & \multicolumn{2}{|c|}{ Silica/Aluminium Ratio } \\
\hline \multicolumn{2}{|l|}{ CEC } & \multicolumn{2}{|c|}{ Cation Exchange Capacity } \\
\hline \multicolumn{2}{|l|}{ FBR } & \multicolumn{2}{|c|}{ Fixed-Bed Reactor } \\
\hline \multicolumn{2}{|l|}{$\mathbf{P F}$} & \multicolumn{2}{|c|}{ Plug Flow } \\
\hline \multicolumn{2}{|l|}{ SBC } & \multicolumn{2}{|c|}{ Slurry Bubble Column } \\
\hline \multicolumn{2}{|c|}{$\mathrm{HNO}_{3}$} & \multicolumn{2}{|c|}{ Concentrated Nitric Acid } \\
\hline $\mathbf{P b}^{2+}$ & {$[\mathbf{P}]$} & Lead ion & Single-Component Lead System \\
\hline $\mathrm{Cu}^{2+}$ & [C] & Copper ion & Single-Component Copper System \\
\hline $\mathbf{F e}^{3+}$ & {$[\mathbf{F}]$} & Iron ion & Single-Component Iron System \\
\hline $\mathrm{Ni}^{2+}$ & [N] & Nickel ion & Single-Component Nickel System \\
\hline $\mathrm{Zn}^{2+}$ & [Z] & Zinc ion & Single-Component Zinc System \\
\hline \multicolumn{2}{|c|}{ [D-xy] } & \multicolumn{2}{|c|}{ Dual-Component System (xy) } \\
\hline \multicolumn{2}{|c|}{ [T] } & \multicolumn{2}{|c|}{ Triple-Component System } \\
\hline \multicolumn{2}{|c|}{ [M] } & \multicolumn{2}{|c|}{ Multi-Component System } \\
\hline \multicolumn{2}{|c|}{ ANALEST } & \multicolumn{2}{|c|}{ Analytical Lab for Environmental Science Research and Training } \\
\hline SOP & & Standard $\mathrm{O}_{1}$ & rating Procedure \\
\hline QDL & & Quantifiabl & Detection Limits \\
\hline$\%-R_{1}$ & & \%-Relative & tandard Deviation \\
\hline $\mathbf{C C}$ & & Correlation & oefficient \\
\hline SR & & Selectivity & atio \\
\hline PFO & & Pseudo-Firs & Order Model \\
\hline PSO & & Pseudo-Sec & id-Order Model \\
\hline IPD & & Intra-Partic & Diffusion Model \\
\hline RDS & & Rate-Deterr & ning Step \\
\hline $\mathbf{B P}$ & & Breakthrou & Point (Breakpoint) \\
\hline EP & & Exhaustion & oint \\
\hline MTZ & & Mass Trans & r Zone \\
\hline C1 & & Column 1 & \\
\hline $\mathbf{C 2}$ & & Column 2 & \\
\hline SC1 & & Sampling C & amber 1 \\
\hline $\mathrm{SC2}$ & & Sampling C & amber 2 \\
\hline MOL & & Sampling N & de (I, II, III) \\
\hline
\end{tabular}




\section{LIST OF SYMBOLS}

\begin{tabular}{|c|c|c|}
\hline$\% \mathbf{R}$ & Percent removal & $(\%)$ \\
\hline$d_{p}$ & Particle size & $(\mathrm{mm})$ \\
\hline $\mathbf{q}_{\mathrm{t}}$ & Sorption capacity of HMI at time $t$ & (meq/g) \\
\hline $\mathrm{C}_{0}$ & Influent HMI concentration in solution & (meq/L) \\
\hline $\mathrm{C}_{\mathrm{t}}$ & Effluent HMI concentration at time $\mathrm{t}$ & (meq/L) \\
\hline $\mathbf{t}$ & Contact time & $(\min )$ \\
\hline $\mathbf{V}$ & Batch-mode sample volume & $(\mathrm{L})$ \\
\hline $\mathbf{M}$ & Zeolite mass & $(\mathrm{g})$ \\
\hline $\mathbf{q}_{180}$ & Sorption capacity at 180 -contact time & (meq/g) \\
\hline$q_{e}$ & Sorption capacity at equilibrium & (meq/g) \\
\hline $\mathbf{k}_{1}$ & PFO rate constant & $\left(\min ^{-1}\right)$ \\
\hline h & initial sorption rate & $(\mathrm{meq} / \mathrm{g} \cdot \mathrm{min})$ \\
\hline $\mathbf{k}_{2}$ & PSO rate constant & $(\mathrm{g} / \mathrm{meq} \cdot \mathrm{min})$ \\
\hline$\beta$ & Elovich constant & $(\mathrm{g} / \mathrm{meq})$ \\
\hline$\alpha$ & Initial sorption rate & $(\mathrm{meq} / \mathrm{g} \cdot \min )$ \\
\hline $\mathbf{k}_{\mathbf{P}}$ & IPD rate constant & $\left(\mathrm{meq} / \mathrm{g} \cdot \mathrm{min}^{1 / 2}\right)$ \\
\hline $\mathbf{C}$ & IPD constant & $(\mathrm{meq} / \mathrm{g})$ \\
\hline $\mathbf{r}_{\mathbf{A}}$ & Reaction rate of reactant & $(\mathrm{meq} / \mathrm{L} \cdot \min )$ \\
\hline$[\mathbf{A}]_{0}$ & Initial reactant concentration & $(\mathrm{meq} / \mathrm{L})$ \\
\hline$[\mathrm{A}]_{\mathrm{t}}$ & Reactant concentration at time $\mathrm{t}$ & (meq/L) \\
\hline $\mathbf{k}_{\mathrm{n}=1}$ & First-Order reaction rate constant & $\left(\min ^{-1}\right)$ \\
\hline $\mathbf{k}_{\mathrm{n}=2}$ & Second-Order reaction rate constant & $(\mathrm{L} / \mathrm{meq} \cdot \min )$ \\
\hline$\eta$ & Column efficiency (degree of saturation) & --- \\
\hline $\mathrm{C}_{\mathrm{BP}}$ & Breakthrough capacity of the bed & (meq/g) \\
\hline $\mathrm{C}_{\mathrm{EP}}$ & Maximum capacity at exhaustion of the bed & (meq/g) \\
\hline $\mathbf{V}_{\mathrm{BP}}$ & Effluent volume collected up to BP & (L) \\
\hline $\mathbf{V}_{\mathrm{EP}}$ & Effluent volume at which the EP is reached in the zeolite bed & $(\mathrm{L})$ \\
\hline$\rho$ & Packing density of the bed & $\left(\mathrm{g} / \mathrm{cm}^{3}\right)$ \\
\hline $\mathbf{H}$ & Bed depth & $(\mathrm{cm})$ \\
\hline $\mathbf{A}$ & Bed cross-sectional area & $\left(\mathrm{cm}^{2}\right)$ \\
\hline $\mathbf{m}_{\mathbf{Z}}$ & Zeolite mass in the bed & (g) \\
\hline$\eta_{\mathrm{BP}}$ & Total amount of HMI ions removed up to BP & (meq) \\
\hline$\eta_{\mathrm{EP}}$ & Total amount of HMI ions removed up to EP & (meq) \\
\hline EBCT & Empty Bed Contact Time & $(\min )$ \\
\hline $\mathbf{v}$ & Feed solution velocity & $(\mathrm{cm} / \mathrm{min})$ \\
\hline d & Column internal diameter & $(\mathrm{cm})$ \\
\hline $\mathbf{Q}$ & Volumetric flow rate & $(\mathrm{mL} / \mathrm{min})$ \\
\hline $\mathbf{v}_{\mathbf{U}}$ & Usage rate & $(\mathrm{g} / \mathrm{L})$ \\
\hline $\mathbf{k}_{\mathbf{T}}$ & Coefficient of permeability & $(\mathrm{cm} / \mathrm{s})$ \\
\hline $\mathbf{V}_{\mathrm{C}}$ & Quantity of water that has discharged and collected from the column & $\left(\mathrm{cm}^{3}\right)$ \\
\hline $\mathbf{L}$ & Column height & $(\mathrm{cm})$ \\
\hline $\mathbf{A}_{\mathbf{c}}$ & Column cross-sectional area & $\left(\mathrm{cm}^{2}\right)$ \\
\hline $\mathbf{H}_{\mathbf{C}}$ & $\begin{array}{l}\text { Constant head of water on the column or the vertical distance } \\
\text { between the feed head level and the column overflow level }\end{array}$ & $(\mathrm{cm})$ \\
\hline $\mathbf{T}$ & Time required to collect $\mathrm{V}_{\mathrm{C}}$ & (seconds) \\
\hline
\end{tabular}




\section{INTRODUCTION}

\subsection{Industrial Wastewater Effluents - Characteristics, Impacts, and Treatment Methods}

Water has been damaged by various anthropogenic sources over the past several decades, and such sources include but are not limited to municipal wastewater treatment plants, agricultural cultivation and fertilization activities, as well as mining and industrial wastewater effluents (Asubiojo and Ajelabi, 2009; Barakat, 2011; Fu and Wang, 2011; Tchounwou et al., 2012; Lata et al., 2015). Surface water, ground water, as well as municipal and industrial wastewater contain many different types of pollutants, such as organics and inorganics (i.e., anionic and cationic ions (heavy metallic ions; HMIs)), causing toxic effects on our ecosystem (Wang and Peng, 2010). Natural water systems are comprised of chemical and physical processes that affect both the distribution and circulation of pollutants. The hydrologic cycle has climatic and seasonal variations, which influences geo-chemical processes and may cause dissolution and chemical reactions with solids, liquids and gases. Anthropogenic interferences associated with industrial processing effluents may cause slight variations in these natural waters, directly causing an influx of pollutants, such as HMIs, which adsorb onto particulates and deposit onto sediments affect the overall balance of metals in the aquatic system (Yabe and de Oliveira, 2003).

Heavy metals are an environmental priority pollutant as they deteriorate water quality, and are a detrimental threat to our ecosystem (Alvarez-Ayuso et al., 2003; Inglezakis and Poulopoulos, 2006, p. 18; Fu and Wang, 2011). Because of their high solubility in the aquatic environment, heavy metals can impact living organisms (microorganisms, fish, and plants) (Kocasoy and Sahin, 2007; Barakat, 2011). Heavy metals are non-biodegradable pollutants which persist and remain intact in the environment (Asubiojo and Ajelabi, 2009) and once entered and transferred through the food chain (Kocasoy and Sahin, 2007), they trigger various diseases and disorders (Erdem et al., 2004; Akcil and Koldas, 2006; Sprynskyy et al., 2006; Inglezakis and Poulopoulos, 2006, p. 18; Acheampong et al., 2010; Barakat, 2011). The effects of heavy metals in wastewater effluents not only impact the environment, but are detrimental to humans also. The impact can be toxic (acute, chronic or sub-chronic), neurotoxic, carcinogenic, 
mutagenic or teratogenic (Acheampong et al., 2009; Akpor et al., 2014). Lead is known as a designated substance, with carcinogenic consequences that affect every organ and system in the human body (OHSA, 2014; Martin and Griswold, 2009; Tchounwou et al., 2012). In addition to lead, nickel and zinc that are dissolved in water are acutely toxic at various levels, and cause numerous health complications; such as cardiovascular and nervous system function conflicts that result in death (Acheampong et al., 2009).

Being part of a very complex and dynamic system, there are many anthropogenic sources and sinks of heavy metals, which can be transported in their dissolved form in water or part of suspended sediments, volatilized to the atmosphere or stored in sediments (Curenature, 2018). The most common source of pollution is through the discharge of wastewater as a by-product of industrial processing. The increased demand for the production of commodities produced by various industries has triggered an accumulation of pollutants (i.e., heavy metals) (Barakat, 2011; $\mathrm{Fu}$ and Wang, 2011; Farouq and Yousef, 2015). Large quantities of wastewaters, residues, and sludge as hazardous wastes are generated, which require extensive waste management (Barakat, 2011). These pollutants put the entire ecosystem at risk, and therefore, it is imperative to remove them prior to their discharge into waterways (Asubiojo and Ajelabi, 2009; Barakat, 2011). Industrial wastewater streams laden with heavy metals include but are not limited to (Barakat, 2011; Fu and Wang, 2011; Tchounwou et al., 2012; Akpor et al., 2014):

- mining operations;

- metal surface treatment processing (i.e., electroplating, electroless depositions, conversioncoating, anodizing-cleaning, milling, and etching);

- inorganic pigment (tanneries, dye) manufacturing;

- petroleum refining;

- batteries and printed circuit board (PCB) manufacturing;

- paper and pesticides processing, as well as;

- fertilizer and wood processing. 
The Canadian mining sector has invested heavily in exploration and development and is among the global leaders in mineral extraction and metal production/processing. As reported by the Ontario Mining and Exploration Directory (2015), there are 32 metal processing (i.e., gold, base metal, iron, platinum group) mines and 43 mines in total in this province alone. In particular, the towns of Timmins and Sudbury ('The Nickel Belt') are associated with industrial wastewater effluents and heavy metal processing pollutants. At just $300 \mathrm{~km}$ apart and in proximity to our great lakes, these industrial sites pose great concern to our revered fresh water source and prompts the discussion of the importance of treatment technology development to remove heavy metals, in order to meet stringent water/wastewater regulations (Kocasoy and Sahin, 2007).

The Great Lakes Water Quality Agreement (GLWQA), which originated in 1972, reflects the continued concern of the future direction and quality of the Great Lakes ecosystem (Rasmussen, 1979). As stated by the Canada-United States Collaboration for Great Lakes Water Quality, the purpose of the agreement is to address current and future challenges in source control, and "...to restore and maintain the chemical, physical, and biological integrity of the Waters of the Great Lakes". The GLWQA has evolved over the years with various amendments $(1978,1983,1987,2012)$ to address the problem of toxic substances in the lakes and the pollution from assorted land use activities, through the development and implementation of stricter water quality goals (Rasmussen, 1979), (water/wastewater) contingency planning and management. In particular, the 1978 GLWQA amendment initiated the concern of heavy metals. According to the International Joint Commission of the United States and Canada for the 1978 GLWQA amendment, the necessity for industry-academia research by means of 'development, interpretation and demonstration of advanced scientific knowledge for the resolution of issues' is stressed. Metals of persistent toxic concern were emphasized, with total concentrations in an unfiltered water sample to not exceed the thresholds to protect aquatic life and raw water for public supply; such concentrations include 10-25, 5, 25, 30, 50, 0.2, and $50 \mathrm{mg} / \mathrm{L}$ for lead, copper, nickel, zinc, arsenic, cadmium, chromium, respectively. As discussed by Rasmussen (1979), the agreement highlights the need for pollution prevention (P2), which would lead to less heavy metal discharge to control at the source of an industrial process. The agreement also introduces the requirements that joint source control research programs be cost-effective as a priority for our Great Lakes. 
Industrial wastewater effluents are treated to meet federal and provincial thresholds by neutralizing free acidity, as well as removing suspended solids and metals (i.e., lead, copper, nickel, zinc, arsenic, cadmium). Provincial requirements are stricter in certain instances, while scientific fact and societal pressure continue to demand further reductions of acceptable metal concentrations for regulation (Dinardo et al., 1991). Based on the MOECC Water Management Policies, Guidelines and Provincial Water Quality Objectives (1994) and the Provincial Water Quality Objectives (PWQO) (MOECC, 2016), the receiving-water effluent requirements for point source discharges to surface waterbodies have been established. Primary emphasis is on the treated effluent discharge limits from pollution point sources, such as industrial wastewater. Treatment technology-based effluent requirements are contained in a range of provincial and federal acts, regulations, guidelines and policies. For industrial point-source discharges, a concentration-based approach was incorporated into provincial effluent requirements on the basis of experience with municipal sewage treatment systems; and revised guidelines were developed for specific industrial sectors, based on the state of treatment technology for those sectors. Before any discharger-specific effluent requirements are specified, the impact of the treatment technology-based effluent on the receiving water body must be reviewed (MOECC, 2016). Water quality considerations take precedence when contaminant discharges exceed the assimilative capacity of the receiving waters, even if the discharged loadings are within the treatment technology-based effluent requirements based on the guidelines, regulations or policies. Receiving-water based effluent requirements also take precedence when ambient levels of toxic contaminants are above acceptable levels (MOECC, 2016). As outlined in Table 1.1, the heavy metal contamination of various anthropogenic influenced wastewater streams are subject to numerous national and international standards. 
Table 1.1 Water Standards - Maximum Contaminant Level of Heavy Metals (mg/L)

\begin{tabular}{|c|c|c|c|c|c|}
\hline Element & $\begin{array}{l}\text { Water Quality } \\
\text { Standards }\end{array}$ & $\begin{array}{c}\text { Drinking } \\
\text { Water }^{\mathrm{x}}\end{array}$ & $\begin{array}{c}\text { UN FAO } \\
\text { Irrigation }\end{array}$ & $\begin{array}{c}\text { US EPA } \\
\text { Standard } \\
\end{array}$ & $\begin{array}{l}\text { Canadian Mining } \\
\text { Effluent Limits }\end{array}$ \\
\hline $\mathrm{pH}$ & $6.5-8.5$ & --- & & & \\
\hline Lead & 0.0025 & 0.015 & --- & $0.015^{\mathrm{c}}$ & 0.20 \\
\hline Copper & 0.009 & 1.3 & 0.20 & $1.3^{\mathrm{c}}$ & 0.30 \\
\hline Iron & --- & --- & 5 & $0.3^{\mathrm{b}}$ & --- \\
\hline Nickel & 0.052 & 0.1 & 0.20 & $0.1^{\mathrm{a}}$ & 0.5 \\
\hline Zinc & 0.117 & $\begin{array}{ll}-- \\
\end{array}$ & 2 & $5^{\mathrm{b}}$ & 0.50 \\
\hline Arsenic & 0.148 & 0.05 & 0.10 & $0.05^{\mathrm{a}}$ & 0.50 \\
\hline Cadmium & 0.0021 & 0.005 & 0.01 & $0.01^{\mathrm{a}}$ & \\
\hline Chromium & 0.027 & 0.1 & 0.10 & $0.10^{\mathrm{a}}$ & \\
\hline \multicolumn{6}{|c|}{$\mathrm{X}_{(\text {Benjamin, } 2010, \text { p.4); }}{ }^{\mathrm{Y}}$ (Rice et al., 2012); ${ }^{\mathrm{Z}}$ (CMJ, 2014) } \\
\hline
\end{tabular}

Innovative technology research and development for the reduction of water/wastewater toxicity has expanded, in order to meet evolving treatment standards caused by the various anthropogenic sources (Lata et al., 2015). Conventional and emerging (Dinardo et al., 1991) treatment technologies considered in the industry include chemical precipitation, ion-exchange, ion-exchange and solvent extraction, ion-exchange and precipitation, adsorption, bio-sorption, coagulation/flocculation, flotation, membrane filtration, ultrafiltration and electro-chemical remediation (Peric et al., 2004; Mohan and Chander, 2006; Han et al., 2006; Barakat, 2011; Fu and Wang, 2011; Margeta et al., 2013). Chemical (lime/limestone; hydroxide, sulphide) precipitation is most widely used (Dinardo et al., 1991; Barakat, 2011), as it is a relatively simple and inexpensive process; HMIs form insoluble precipitates, separated by sedimentation or filtration, where the treated water is discharged or reused. However, it is not a very economical process due to slow metal precipitation, poor settling, the aggregation of metal precipitates, and the long-term environmental impacts of managing and processing of residual chemicals and sludge (Barakat, 2011; Fu and Wang, 2011). 
Various treatment technologies for the removal of heavy metals from industrial wastewater have focused on physico-chemical removal processes (Barakat, 2011). Among these, sorption (adsorption/ion-exchange) (Helfferich, 1962; Inglezakis and Poulopoulos, 2006) is considered as a promising treatment method (Motsi et al., 2011; Wang et al., 2009). These findings are also supported by Margeta et al. (2013), which highlight the removal efficiency of metal ions by natural zeolite from various sources of wastewater, and shows that sorption is superior compared to standard physical/chemical techniques. Industry has developed an interest for exploring sorption as a wastewater treatment method (Wang et al., 2009; Motsi et al., 2011), based on demonstrated heavy metal removal efficiency, industrial viability (ease of implementation), costeffectiveness (Anari-Anaraki and Nezamzadeh-Ejhieh, 2015; Borandegi and NezamzadehEjhieh, 2015; Xu et al., 2013) (the use of naturally abundant materials) and environmental sustainability (regenerative potential and sludge-free operation) (Lata et al., 2015).

Ion-exchange is considered a very successful removal process for the removal of heavy metals from industrial wastewater effluent, associated with high treatment capacity, high removal efficiency and fast kinetics (Barakat, 2011; Fu and Wang, 2011). Ion-exchange has demonstrated both technical and economic feasibility of metal recovery from acid mine drainage, and is also a significant method for handling wastewaters in the metal finishing industry (Dinardo et al., 1991). Adsorption is also an effective and economic method, with design and operation flexibility, producing high-quality treated effluent. As a reversible process, this leads to the potential to regenerate the sorbent material (Fu and Wang, 2011). There are various advantages associated with physico-chemical treatment of inorganic effluents, which include being a relatively rapid process, with ease of operation and control, accommodates variability in input loads and flow (seasonal flows and complex discharge), as well as requiring lower space and installation costs. Drawbacks of this treatment method include higher operational and sludge disposal handling expenditures, which can be offset with the use of low-cost adsorbent materials (Barakat, 2011). 


\subsection{Selection of Sorbent Material - Natural Zeolite Mineral Characteristics}

Governmental policies and initiatives, such as the GLQWA, have impelled the continued interest by researchers to find cost-effective adsorbent material alternatives to replace conventionally considered and costly granulated activated carbon (GAC). Existing literature in the investigation of various alternatives for the uptake of heavy metals from industrial wastewater include natural minerals such as zeolites and clay minerals (i.e., bentonite, kaolin), biomaterials (i.e., biomass, moss, peat, sawdust), as well as agricultural wastes and industrial byproducts (i.e., fly ash) (Babel and Kurniawan, 2003; Gunay et al., 2007; Wang and Peng, 2010; Fu and Wang, 2011). Among these different sorbent materials, zeolites appear as one of the most promising to perform metal purification processes (Alvarez-Ayuso et al., 2003). Zeolites are a low-cost, globally abundant natural mineral (Kocasoy and Sahin, 2007); considered relatively inexpensive (\$0.03-0.12/kg-USD) compared to industrial grade GAC (\$20-22/kg-USD); depending on the quality of the materials (Babel and Kurniawan, 2003).

Natural zeolites have garnered particular research interest due to their co-existing molecular sieve action, ion-exchange and catalytic properties (Wang et al., 2009; Tsitsishvili, 1988; Nezamzadeh-Ejhieh and Shirzadi, 2014), and are applied worldwide in various industries, which include agriculture, soil remediation, and energy (Wang, 2010), as well as the wastewater treatment industry. Wang and Peng (2010) reported that the global consumption of zeolite has grown from 3.98Mt in 2009 to reach 5.5Mt in 2010, and is on the rise. Among the 40 known types of natural zeolites, clinoptilolite is well-documented and relatively abundant, found in large deposits all over the world (Wingenfelder et al., 2005; Wang and Peng, 2010), and is extensively used on a global scale (Wang and Peng, 2010; Xu et al., 2013) in various sorption treatment studies of industrial effluent. Over the past decade, extensive research of natural zeolites has resulted in a substantial number of patents. As reported by Margeta et al. (2013), over 410 patents and well over 2,000 scientific research efforts have materialized for that of clinoptilolite alone. Due to its global abundance and cost-effectiveness, mineral zeolite has attracted a high level of interest in its use in future water treatment technology development by industrial wastewater processing sectors. 


\subsubsection{Chemical Composition and Structure}

Zeolite is a hydro-aluminosilicate (Tsitsishvili, 1988) crystalline and is a member of the tecto-silicates group, similar to quartz and feldspar minerals (Mumpton, 1977). With a 'honeycomb-like structure' (Jha and Sigh, 2016), it is composed of a three-dimensional framework built of primary building units (tetrahydra) of silicon-oxygen and aluminum-oxygen, which encompass complex rings that create an open homogenous microporous structure of voids and channels (Mumpton, 1977; Tsitsishvili, 1988). The structure of zeolite contains cations $\left(\mathrm{Na}^{+}\right.$, $\mathrm{Ca}^{2+}, \mathrm{Mg}^{2+}, \mathrm{K}^{+}$) (co-ions), and is represented by the general empirical formula shown in Equation 1.1 (Mumpton, 1977; Wang, 2010):

$$
\mathrm{M}_{\mathrm{x} / \mathrm{n}}\left(\mathrm{Al}_{\mathrm{x}} \mathrm{Si}_{\mathrm{y}} \mathrm{O}_{2(\mathrm{x}+\mathrm{y})}\right) \cdot \mathrm{pH}_{2} \mathrm{O}
$$

where $\mathrm{M}$ is any alkali or alkaline earth cation ( $\mathrm{Na}, \mathrm{K}, \mathrm{Li}$ and/or $\mathrm{Ca}, \mathrm{Mg}, \mathrm{Ba}, \mathrm{Sr}$ ), $\mathrm{n}$ is the cation charge, and the constant ratios of $y / x$ and $\mathrm{p} / \mathrm{x}$ ranging from 1-6 and 1-4, respectively. Exchangeable cations are represented as the ions in the first set of parentheses within the unit-cell formula; the ions in the second set of parentheses is $(x+y)$, which refers to the oxygen content; which make up the tetrahedral framework. The ratio of the base to alumina is always equal to the value of $y$. As a true tectosilicate, oxygen is connected to either a silicon or an aluminum ion with a ratio of ( $\mathrm{Al}+\mathrm{Si}): \mathrm{O}$ of 1:2. The presence of silicon ions is greater than the tetrahedral aluminum ions, with the $\mathrm{SiO}_{2}$ : $\mathrm{Al}_{2} \mathrm{O}_{3}$ ratio greater than 2:1 (Mumpton, 1977). Quadrivalent silicon is replaced by trivalent aluminum, creating a positive charge deficiency to become negatively charged. A balance among charges is achieved through the addition of mono- and di-valent cations (Mumpton, 1977). The cations (or co-ions) which leave the zeolite's framework are replaced by an equivalent amount of counter-ions (HMIs). In order to meet its electro-neutrality requirement, when the counter-ion moves out into solution, the zeolite is left with an electric surplus charge to be compensated by taking up another counter-ion; the total counter-ion content (in equivalents) remains constant, regardless of ionic composition (Helfferich, 1962, p. 250).

Significant properties of zeolite, which vary depending on the source and type, include: physical (bulk density, specific gravity, CEC, SSA, void volume, pore radius), chemical (LOI, $\mathrm{Si} / \mathrm{Ai}$ ratio), adsorption/ion-exchange ( $\mathrm{Si} / \mathrm{Al}$ ratio, selectivity), mineralogical and morphological (XRD, SEM/EDS), thermal (temperature resistance, stability, conductivity, heat capacity), acidic 
stability (electronegativity equalization), crystal structure (chain, sheet, framework), framework structure (Si/Al ratio; CEC, channel dimensions, void volume, specific gravity) and surface structure (hydrophobic, hydrophilic) (Jha and Sigh, 2016). The conjugated pairs in cation exchanged zeolites are Lewis acids (extra framework protons cations) and Lewis bases (framework oxygens), where the alkali exchanged zeolites are designated as basic zeolites. The Lewis acidity and Lewis basicity in cation exchanged zeolites are affected by various factors, including the $\mathrm{Si} / \mathrm{Al}$ ratio, bond angles and lengths, the location of $\mathrm{Al}$, the crystallographic sitting of oxygen, as well as the electronegativity of the framework (Huang et al., 1995); the electronegativity referring to the tendency of an atom to attract electrons (Benjamin, 2010, p. 227). The Si/Al ratio influences the amount and distribution of the Si-O-Al groups within the crystalline structure. The greater the ratio, the more Si-O-Si than Si-O-Al linkages, which change the geometrical structure (bond angles and lengths around acid sites) of the zeolite. This influences the degree of $\mathrm{H}^{+}$adsorption selectivity and electrostatic potential and binding interaction strength of cations (i.e., HMI) on negative charge sites in the crystalline framework (Jha and Sigh, 2016). The Si/Al ratio in clinoptilolite is relatively high (4.25-5.25) (Breck,1974; Ouki and Kavanagh, 1997), which corresponds to a low volumetric capacity and relatively weak ionic field within the structure; such that the electrostatic interactions are not as significant as the hydration free energy. Therefore, the metals with the highest free energy of hydration (i.e., lead; $-357.8 \mathrm{kcal} / \mathrm{g} \cdot \mathrm{ion}$ ) (Inglezakis et al., 2003) should prefer to remain in dissolved form. The selectivity order is influenced by both physico-chemical and stereo-chemical factors (i.e., hydrated radii, cation hydration enthalpy, and micropore space requirements). The rejection of water molecules is related to the hydration enthalpies of the cations, which may explain the high selectivity of lead (Inglezakis et al., 2005). The analysis of natural zeolites for the treatment of mixed metal-contaminated effluents by Ouki and Kavanagh (1997) showed that based on the hydration energies, the selectivity series for the metals considered should be $\mathrm{Pb}>\mathrm{Cd}>\mathrm{Co}>\mathrm{Zn}>$ $\mathrm{Cu}$; with the greater selectivity of lead in keeping of the research presented in the Dissertation document.

There is a wide variety of zeolites, which depend on the environment from which they are derived. Their behaviour is dependent upon various factors, including the framework structure, cation species' nature (i.e., size, shape, and charge), the external electrolyte shape and solution ionic charge, cation species and solution concentration, as well as temperature (Mumpton, 1977; 
Wang, 2010). The clinoptilolite zeolite mineral type is related to the heulandite category, as part of Group $7\left(\mathrm{~T}_{10} \mathrm{O}_{20}\right.$ units), with greater potassium and slightly greater silica contents. Like heulandite, its structural organization are sheets that are connected by bonds, of which are comparatively separated in a monoclinic crystal structure; contains $39 \%$ void volume, as well as an approximate specific gravity and ion-exchange capacity of 2.16 and $2.54 \mathrm{meq} / \mathrm{g}$, respectively (Mumpton, 1977). Its framework structure consists of three channels. Channels A and B are 12membered $(0.75 \times 0.31 \mathrm{~nm})$ and 8 -membered $(0.46 \times 0.361 \mathrm{~nm})$ tedrahedral rings respectively, are parallel to each other. The third channel $\mathrm{C}$ is an 8 -membered $(0.47 \times 0.28 \mathrm{~nm})$ tedrahedral ring that intersects channels A and B (Çakıcğlu-Ozkan and Ülkü, 2008). Figure 1.1 provides a view of the clinoptilolite structure, and its relationship with a counter-ion (i.e., HMI) to its framework.

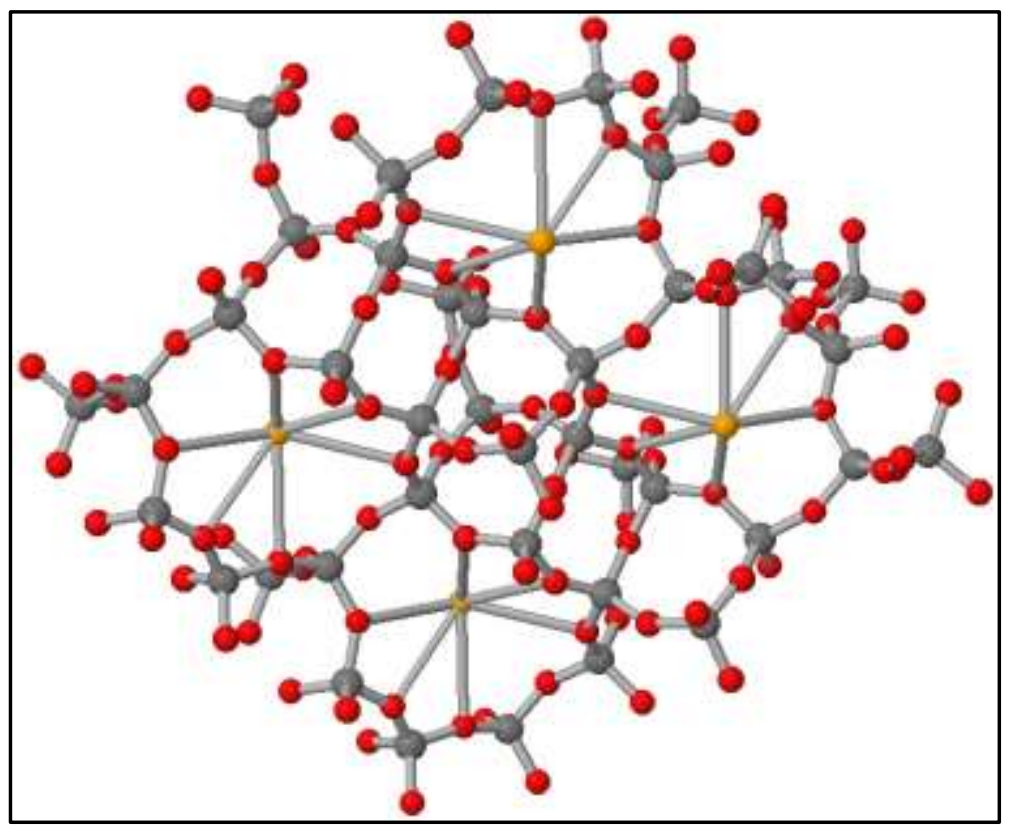

Figure 1.1 Relationship of the Clinoptilolite Framework and a Trapped Cation (adapted from University of Liverpool, 2017)

The structure of zeolite is divided into three components (Mumpton, 1977; Wang, 2010): (1) alumino-silicate framework, (2) interconnected void spaces in a framework containing exchangeable cations (co-ions), and (3) zeolitic water (water molecules as an occluded phase) present at 10-20\% (Margeta et al., 2013) of the dehydrated phase of the natural zeolites' structure. In addition, zeolite is comprised of various phases: (1) zeolite and the external sorbate solution, (2) molten salt and zeolite at equilibrium, and (3) the 'solvent vapour' (water), a thermodynamic reference point with profound effect on the reaction (i.e., attainment rate and exchange position at equilibrium). There are both thermodynamic and kinetic aspects to ion- 
exchange, being a stoichiometric process between the phases to maintain electro-neutrality. The chemical reaction involves cations initially present in separate phases moving between two phases until equilibrium within each phase is achieved (Bekkum et al., 1991). Available exchange sites have been referred to as 'two-way traffic in a one-way street' (Helfferich, 1962, p. 186). The amount of solvent that the crystalline is able to uptake depends on the internal volume, defined by the lattice channels' width and intra-crystalline voids, and on the volume occupied by counter-ions found in solution.

The co-ions within the framework significantly influence the sorption and thermal properties of the zeolite (Çakıc̆̆lu-Ozkan and Ülkü, 2008; Yörükoğulları et al., 2010). Three water types are recognized in zeolite: (1) water with crystal-water-like bonds, (2) water bound to the lattice by $\mathrm{OH}-$ bonds, and (3) 'typical'/zeolitic water (able to freely move into and out of the lattice without disruption (Van Reeuwijk, 1974). Water molecules occupy the large central cavities and entry channels (Ovsyuk and Goryaœnov, 2006; Çakıcğlu-Ozkan and Ülkü, 2008), which form hydration spheres around the exchangeable cations. The crystal structure of zeolite possesses a considerable void space within its simple, polyhedral building blocks and within larger frameworks formed by several polyhedral blocks. The pore sizes differ, ranging from 3-10 $\dot{\mathrm{A}}$ (Breck, 1974; Jha and Sigh, 2016), depending on the structure, channel and cavity systems, as well as effective entry opening (Mumpton, 1977). A considerable amount of water is given off continuously and reversibly by heating from room temperature to approximately $350^{\circ} \mathrm{C}$. When water is removed, cations fall back into positions on the inner surface of channels and central cavities of the zeolite structure. Thermal/Dehydration treatment of zeolite is an endothermic process (Mumpton, 1977), which significantly impacts the mineralogical properties and specific sieve action requirements of the mineral; sorptive properties of the mineral depending greatly on its cell dimensions. Activation of 'stable' species (i.e., clinoptilolite) is often accompanied by discernible lattice deformations and phase transformations. The leading mechanism of the dehydration process refers to the reaction enthalpy; the sum of the energies required for the subreactions involved (i.e., volatilization of water (breaking bonds with cations), diffusion of water through the channels, re-arrangement of lattice), and depends on the temperature and water vapour pressure of the process (Van Reeuwijk, 1974). Dehydrated zeolite typically achieves void volumes of up to $50 \%$ (Mumpton, 1977). Given that water is selectively adsorbed by natural zeolite, it slows the sorption rate of the other molecules (Yörükoğulları et al., 2010). 
In addition to global abundance, existing literature has shown that natural zeolites are resilient towards chemical, biological, mechanical or thermal modifications (Margeta et al., 2013). Most importantly, natural zeolites have a relatively high cation exchange capacity (CEC) (depending on its Si/Al ratio) (Nezamzadeh-Ejhieh and Shirzadi, 2014) and good selectivity (Inglezakis et al., 2002; Gunay et al., 2007), which are strong attributes for the removal of heavy metals (Ersoy and Celik, 2002).

\subsubsection{Sorption - Adsorption and Ion-Exchange Capacity}

Natural zeolites exhibit high adsorption of heavy metals in aqueous solutions, due to their combined adsorption and ion-exchange properties (Wang and Peng, 2010). They are able to neutralize acidic wastewater, as well as be regenerated and reused (Kocasoy and Sahin, 2007); which make them a favourable material for industrial wastewater loaded with heavy metals (Babel and Kurniawan, 2003). The term sorption refers to every type of capture of a substance from the external surface of solids, liquids or mesomorphs as well as from the internal surfaces of porous solids or liquids. It is a reversible phenomenon that depends on factors such as the molecular size, swelling pressure, sieve action, solution concentration, and temperature (Helfferich, 1962) and is classified as (Inglezakis and Poulopoulos, 2006, p. 31):

- Physical (Physiosorption) - no exchange of electrons occurs but rather intermolecular attractions between favourable energy sites and is independent of the electronic properties of the molecules involved;

- Chemical (Chemisorption) - exchange of electrons between specific surface sites and solute molecules, forming a chemical bond; stronger and more stable than physiosorption; and

- Electrostatic (Ion-Exchange) - attractions between ions and charged functional groups.

Adsorption, in particular, is referred to as the separation process where specific components of one phase of a fluid transfers onto the surface of a solid adsorbent. Adsorptive molecules transport through macropores to the mesopores and finally enter the micropores, where the micropores establish a majority of the internal surface and represent most of the total pore volume (Inglezakis and Poulopoulos, 2006, p. 33). This process involves molecules from a liquid phase that bind in a condensed layer onto a solid surface. The quantity adsorbed depends on temperature, pressure and composition of the solution (Masel, 1996). Zeolites possess unique adsorption properties, characterized as a synthetic molecular sieve. Large central cavities and 
access channels are filled with water molecules to form hydration spheres around the exchangeable cations at normal conditions. Conditioning is achieved when water is removed by heating the material to $350-400^{\circ} \mathrm{C}$ for a few hours to overnight. The molecules with effective cross-sectional diameters small enough to pass through the channels are promptly adsorbed in both the dehydrated channels and cavities. Molecules that are too large to pass through the channels are excluded, demonstrating its molecular sieving property (Mumpton, 1977).

Ion-exchange capacity of zeolite is defined by degree of substitution of aluminum for silicon in the framework structure; the exchange of ions between a liquid and solid phase. The greater the substitution, the greater charge deficiency, such that a higher number of alkali or alkaline earth cations become required for electrical neutrality (Mumpton, 1977). Pure ion-exchange occurs at equimolar concentration, such that the ratio of the $\mathrm{HMI}(\mathrm{s})$ bound to the sorbent material and released should be equal to unity (Mohan and Chander, 2006). Ion-exchangers, such as zeolites, are able to uptake charged ions from the solution and release an equivalent amount of other ions into the solution. This ability is attributed to the charged framework in specific locations in the solid structure or in functional groups. This charge is balanced by the counterions that move in the framework and are replaced by other ions of equal charge (Inglezakis and Poulopoulos, 2006, p. 34). A major attribute of zeolites is their ability to exchange ions with external medium through an iso-morphous approach. This is expressed by the following Equation 1.2 (Wang, 2010).

$$
\mathrm{z}_{\mathrm{B}} \mathrm{A}_{\mathrm{A}}^{+}+\mathrm{z}_{\mathrm{A}} \mathrm{BL}_{\mathrm{Z}_{\mathrm{B}}} \rightleftharpoons \mathrm{z}_{\mathrm{A}} \mathrm{B}^{\mathrm{Z}_{\mathrm{B}}^{+}}+\mathrm{z}_{\mathrm{B}} \mathrm{AL}_{\mathrm{Z}_{\mathrm{A}}}
$$

where $\mathrm{z}_{\mathrm{A}}$ and $\mathrm{z}_{\mathrm{B}}$ are the valences of respective cations, and $\mathrm{L}$ is the proportion of the zeolite framework holding unit negative charge. The solid phase (exchanger) is charged and balanced by the ions of an opposing charge, known as counter-ions. The exchanger containing ions $\mathrm{A}$ is immersed in the liquid phase (solution) containing ions B. Diffusion takes place due to the considerable concentration imbalance between the exchanger and solution; ions $\mathrm{A}$ diffuses out from and $\mathrm{B}$ diffuses into the exchanger. Equilibrium is established once the ions stabilize the existing concentration variation. Most importantly, it is the active charge of the solid phase that is directly related to the ion-exchange capacity of the solid phase in the system. The fact that the structure of natural zeolite contains exchangeable cations $\left(\mathrm{Na}^{+}, \mathrm{Ca}^{2+}, \mathrm{Mg}^{2+}, \mathrm{K}^{+}\right)$(co-ions) which are relatively innocuous to the ecosystem promotes its 
potential in tertiary treatment of industrial wastewater (Vukojevic Medvidovic et al., 2006; Gaikwad et al., 2011).

The removal of HMIs is attributed to both adsorption (on the surface of the sorbents' micropores) and ion-exchange (through the sorbents' framework pores and channels) mechanisms (Curkovic et al., 1997). Both are a mass transfer process from a fluid to a solid phase, and the total charge sorbed and desorbed is explicitly the same as required by the electroneutrality principle (Helfferich, 1962; Inglezakis and Poulopoulos, 2006). It is important to note that ion-exchange is very similar to sorption; however, it is a stoichiometric process where for every ion removed, another ion is replaced in the release into solution (Inglezakis and Poulopoulos, 2006, p. 34). For practical applications, it is generally accepted to collect adsorption and ion-exchange as sorption for a unified treatment process (Helfferich, 1962; Inglezakis and Poulopoulos, 2006).

Inglezakis (2005) reviews theoretical considerations and experimental methods, and distinguishes several types of capacity with respect to zeolite systems. The research demonstrates the need for unified terminology and standardized methodology in regards to zeolite capacity studies, and enforces that further contributions must be theoretically investigated and experimentally proven. The complexity in addressing 'real exchange capacity' when determining the concentration of exchangeable cations present in the sorbent is discussed. Inglezakis (2005) concludes by bringing the attention to metals, where they are not only exchanged but also adsorbed and precipitated onto the exchanger's surface. This presents a greater challenge when addressing zeolite capacity, such that it becomes the sum of both the active ion-exchange and adsorption sites. Inglezakis (2005) highlights the capacity terms defined by IUPAC as follows:

- Theoretical (Specific) - number of ionogenic groups per specified amount ion-exchanger;

- Apparent/Effective - number of exchangeable counter-ions per specified amount of material;

- Practical (Specific) - total amount ions taken up per gram ion-exchanger under specific (given) conditions; may be lower than the theoretical amount if conditions prevent full exchange;

- Useful - when equilibrium is unattainable, and;

- Breakthrough - utilized in column operations. 
The theoretical exchange capacity refers to the maximum exchange level, and is expressed by the amount of exchangeable cations in a specific, fixed quantity of the mineral; which can be derived from its elemental composition (Oter and Akcay, 2007). Specifically to zeolites, there are no separate ionogenic groups. The tetrahedral lattice of $\mathrm{SiO}_{4}$ and $\mathrm{AlO}_{4}$ are common with their oxygen atom, providing a trivalent aluminum negative electric charge. This charge is balanced by alkali and alkaline earth cations and do not occupy fixed positions, but rather are free to move in the lattice framework such that the counter-ions are replaced by other cations. Consequently, the negative charge of zeolite is not localized but rather uniformly distributed and constant, known as the 'framework charge' (Inglezakis, 2005). Also expressed by Oter and Akcay (2007), the negative charges are compensated for by incorporating the cations (co-ions), which are not part of the actual tetrahedral framework, but rather exist in the internal channels. As the crystalline lattice absorbs the solution into its channels, one cation may be exchanged for another without influencing the mineral structure or electro-neutrality.

The apparent/effective capacity (including useful and breakthrough capacities; Inglezakis, 2005) is of more practical importance, as it refers to the amount of exchangeable cations of zeolite contained in a specific amount of the material (Oter and Akcay, 2007), which balance the 'framework charge' (Inglezakis, 2005). This capacity is dependent upon the experimental conditions, which include: (1) liquid-volume-to-solid-mass ratio (dosage), (2) batch systems (agitation rate and contact time), (3) column systems (volumetric flow rate and breakpoint concentration), and (4) temperature and normality (ionic concentrations) (Inglezakis, 2005). This points to the unique nature of natural zeolites and thereby generates a necessity to redefine the term 'capacity'. As such, Inglezakis (2005) proposes the following capacity terminology shown in the Figure 1.2. Based on the proposed terminology, the analysis showed the following capacity series: $T E C \geq R E C \geq M E L \geq O C \geq E C, B C$. The research conducted has applied particular experimental conditions, contributing to the overall unified terminology predicament of the practical capacity category. 


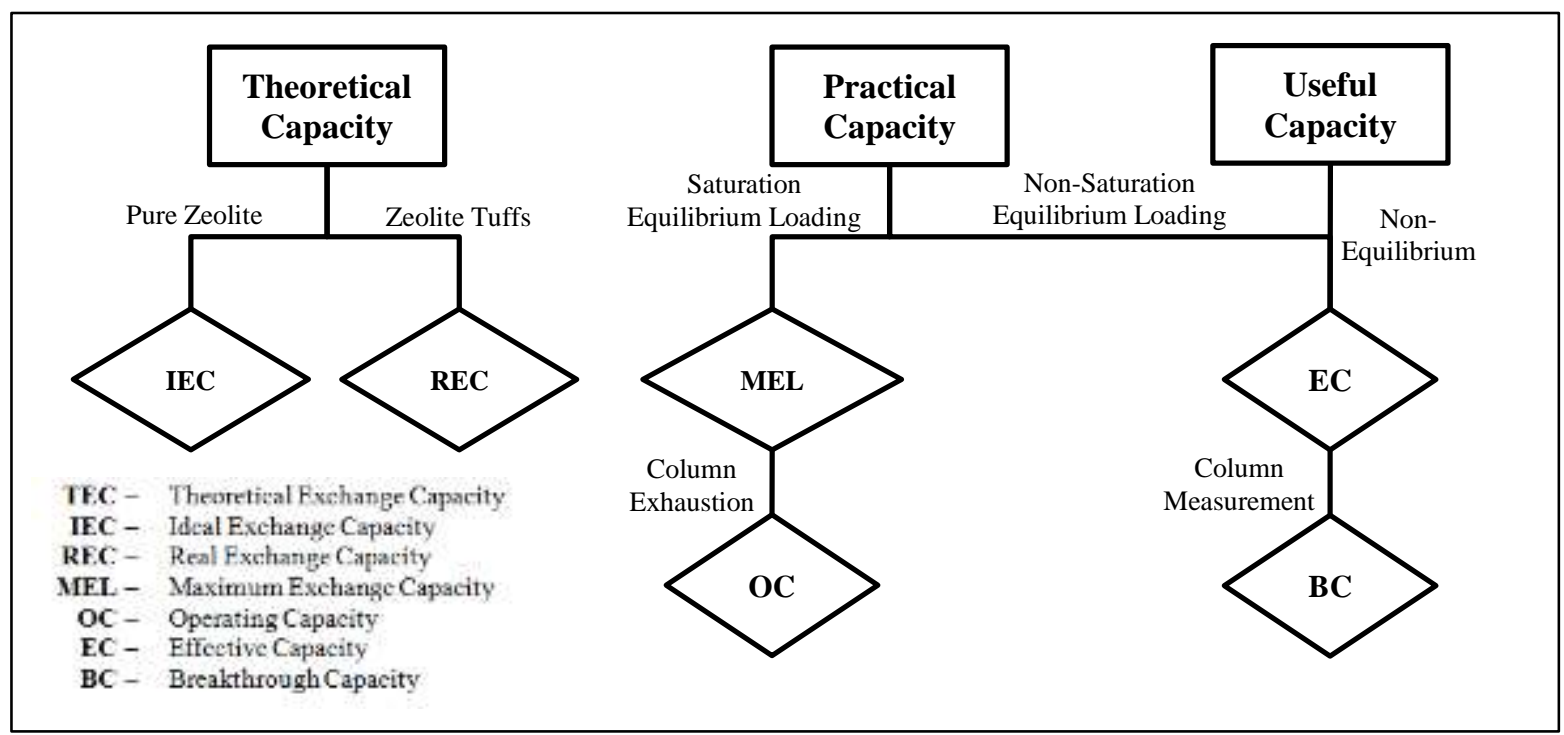

Figure 1.2 Capacity Terminology Considerations (adapted from Inglezakis, 2005)

The challenge to define 'real exchange capacity' is due to associated impurities and subsequent experimental errors. The inconsistency of experimental procedures and detection methods among researchers generates considerably inadequate comparability for a given system. Consequently, the overall underlying message to the research stresses the importance of a proper experimental procedure and the further development of unified terminology. The research presented in the Dissertation should be recognized as a 'proof-of-concept', as it contributes to the collection of data to further advance the understanding of these terminologies. 


\subsection{Selection of Experimental Influent Stock}

The influent stock is of great importance to the experimental program in this research project. Existing literature targeting heavy metal removal introduce the discussion with an emphasis on the importance of the various industrial wastewater effluent streams previously described, which evidently all contain diverse constituents in solution. Previous studies have investigated the treatment capacity of zeolites with synthetic simple solute solutions or simulated acidic mine waters that are prepared by combining and dissolving various heavy metal analytical grade salts, with anions such as chloride, nitrate or sulphate, with either tap water or distilled water. However, research in the remediation of actual industrial wastewater is still very limited (Xu et al., 2013).

Table 1.2 provides a summary of several relevant literatures that showcase the various types of influent stock investigated in the removal of HMIs. In review, there is a distinct variety in the source and type of sorbent material used, experimental configurations, analytical methodology as well as the targeted heavy metals of interest. Although diverse in the design of their respective experimental programs, these literatures have concluded a comparatively consistent HMI removal trend. The review by Babel and Kurniawan (2003) reports a trend of $\mathrm{Pb}>\mathrm{Cd}>\mathrm{Cu}>\mathrm{Co}$ $>\mathrm{Cr}>\mathrm{Zn}>\mathrm{Ni}>\mathrm{Hg}$, while Wingenfelder et al. (2005) states $\mathrm{Pb}>\mathrm{Cu}, \mathrm{Zn}>\mathrm{Cd}, \mathrm{Sr}>\mathrm{Co}$ and $\mathrm{Pb}>$ $\mathrm{Cd}>\mathrm{Cs}>\mathrm{Cu}>\mathrm{Co}>\mathrm{Cr}>\mathrm{Zn}>\mathrm{Ni}>\mathrm{Hg}$; additional trends summarized in the Table 1.2. This consistency of trends contributes to the persistent great interest by the scientific community, and importance of the methodical combination of the selected heavy metals investigated by the study presented in the Dissertation document. 
Table 1.2 Existing Trends in Influent Stock, Zeolite Material and Heavy Metals of Interest

\begin{tabular}{|c|c|c|c|c|c|}
\hline $\begin{array}{l}\text { Influent Stock } \\
\text { Solution }\end{array}$ & Zeolite & \multicolumn{2}{|c|}{ HMI } & Removal Trend & Reference \\
\hline \multirow{15}{*}{$\begin{array}{l}\text { Synthetic } \\
\text { Simple Solute }\end{array}$} & $\begin{array}{c}\text { American } \\
\text { 85-95\% Clinoptilolite }\end{array}$ & $\begin{array}{l}\text { Lead } \\
\text { Iron } \\
\text { Copper }\end{array}$ & $\begin{array}{l}\text { Zinc } \\
\text { Nickel }\end{array}$ & $\begin{array}{c}\mathrm{Pb} \gg \mathrm{Fe}>\mathrm{Cu} \\
>\mathrm{Zn}>>\mathrm{Ni}\end{array}$ & This Study \\
\hline & $\begin{array}{c}\text { American Zeolite } \\
\text { (Clinoptilolite and Chabazite) }\end{array}$ & $\begin{array}{l}\text { Lead } \\
\text { Copper } \\
\text { Zinc } \\
\text { Nickel }\end{array}$ & $\begin{array}{l}\text { Cadmium } \\
\text { Chromium } \\
\text { Cobalt }\end{array}$ & $\begin{array}{l}\mathrm{Pb}>\mathrm{Cu}>\mathrm{Cd}>\mathrm{Zn} \\
\quad>\mathrm{Cr}>\mathrm{Co}>\mathrm{Ni}\end{array}$ & Ouki and Kavannagh (1999) \\
\hline & $\begin{array}{c}\text { Mexican } \\
\text { 70\% Clinoptilolite }\end{array}$ & $\begin{array}{l}\text { Lead } \\
\text { Cadmium }\end{array}$ & $\begin{array}{l}\text { Chromium } \\
\text { Phenol }\end{array}$ & $\mathrm{Pb}>\mathrm{Cd}>\mathrm{Cr}$ & Vaca-Mier et al. (2001) \\
\hline & $\begin{array}{c}\text { Brazilian } \\
\text { Zeolite (Scolecite) }\end{array}$ & $\begin{array}{l}\text { Copper } \\
\text { Zinc } \\
\text { Lead }\end{array}$ & $\begin{array}{l}\text { Nickel } \\
\text { Cobalt } \\
\text { Cadmium }\end{array}$ & $\begin{array}{c}\mathrm{Cu}>\mathrm{Zn}>\mathrm{Pb} \\
>\mathrm{Ni}>\mathrm{Co}>\mathrm{Cd}\end{array}$ & Bosso and Enzweiler (2002) \\
\hline & $\begin{array}{c}\text { Greek Zeolite } \\
\text { (Natural Clinoptilolite) }\end{array}$ & $\begin{array}{l}\text { Copper } \\
\text { Cadmium } \\
\text { Chromium }\end{array}$ & $\begin{array}{l}\text { Nickel } \\
\text { Zinc }\end{array}$ & $\begin{array}{c}\mathrm{Cu}>\mathrm{Cr}>\mathrm{Zn} \\
>\mathrm{Cd}>\mathrm{Ni}\end{array}$ & Alvarez-Ayuso et al. (2003) \\
\hline & $\begin{array}{c}\text { Greek } \\
\text { Clinoptilolite }\end{array}$ & $\begin{array}{l}\text { Lead } \\
\text { Copper }\end{array}$ & $\begin{array}{l}\text { Iron } \\
\text { Chromium }\end{array}$ & $\mathrm{Pb}>\mathrm{Cr}>\mathrm{Fe}>\mathrm{Cu}$ & $\begin{array}{c}\text { Inglezakis et al. } \\
(2002,2003,2004)\end{array}$ \\
\hline & $\begin{array}{l}\text { Turkish } \\
\text { 70\% } \\
\text { Clinoptilolite }\end{array}$ & $\begin{array}{l}\text { Cobalt } \\
\text { Copper }\end{array}$ & $\begin{array}{l}\text { Zinc } \\
\text { Manganese }\end{array}$ & $\mathrm{Co}>\mathrm{Cu}>\mathrm{Zn}>\mathrm{Mn}$ & $\begin{array}{c}\text { Erdem et al. (2004) } \\
\text { Wang and Peng (2010) }\end{array}$ \\
\hline & $\begin{array}{c}\text { Ukrainian } \\
75 \% \text { Clinoptilolite } \\
\end{array}$ & $\begin{array}{l}\text { Lead } \\
\text { Copper }\end{array}$ & $\begin{array}{l}\text { Nickel } \\
\text { Cadmium }\end{array}$ & $\mathrm{Pb}>\mathrm{Cu}>\mathrm{Cd}>\mathrm{Ni}$ & Spryskyy et al. (2006) \\
\hline & $\begin{array}{c}\text { Sardinian } \\
\text { 40-70\% Clinoptilolite }\end{array}$ & $\begin{array}{l}\text { Lead } \\
\text { Copper }\end{array}$ & $\begin{array}{l}\text { Cadmium } \\
\text { Zinc }\end{array}$ & $\mathrm{Pb}>\mathrm{Cu}>\mathrm{Cd} \cong \mathrm{Zn}$ & Cincotti et al. (2006) \\
\hline & Lignite & $\begin{array}{l}\text { Iron } \\
\text { Manganese } \\
\text { Zinc } \\
\end{array}$ & & $\mathrm{Fe}>\mathrm{Zn}>\mathrm{Mn}$ & Mohan and Chander (2006) \\
\hline & $\begin{array}{c}\text { Turkish } \\
\text { Clinoptilolite }\end{array}$ & Lead & & --- & Gunay et al. (2007) \\
\hline & $\begin{array}{c}\text { Turkish (Aegean) } \\
\text { Clinoptilolite } \\
\end{array}$ & $\begin{array}{l}\text { Copper } \\
\text { Iron } \\
\text { Zinc } \\
\end{array}$ & & $\mathrm{Cu}>\mathrm{Fe}>\mathrm{Zn}$ & Kocasoy and Sahin (2007) \\
\hline & $\begin{array}{c}\text { Turkish Zeolite } \\
\text { (Clinoptilolite and Heulandite) }\end{array}$ & $\begin{array}{l}\text { Lead } \\
\text { Zinc }\end{array}$ & $\begin{array}{l}\text { Copper } \\
\text { Nickel }\end{array}$ & $\mathrm{Pb}>\mathrm{Zn}>\mathrm{Cu}>\mathrm{Ni}$ & $\begin{array}{l}\text { Oter and Akcay (2007) } \\
\text { Wang and Peng (2010) }\end{array}$ \\
\hline & Turkish Zeolite & Copper & & $\begin{array}{c}-- \\
-1\end{array}$ & Aydin and Saygili (2009) \\
\hline & $\begin{array}{c}\text { Serbian } \\
\text { Clinoptilolite }\end{array}$ & $\begin{array}{l}\text { Lead } \\
\text { Zinc }\end{array}$ & & $\mathrm{Pb}>\mathrm{Zn}$ & $\begin{array}{c}\text { Nuic, Peric, Trgo, } \\
\text { Vukojevic Medvidovic } \\
(2006-2016)\end{array}$ \\
\hline $\begin{array}{c}\text { Simulated } \\
\text { Acidic Mine Water }\end{array}$ & $\begin{array}{c}\text { Slovakian } \\
\text { Zeolite } \\
\end{array}$ & $\begin{array}{l}\text { Lead } \\
\text { Cadmium }\end{array}$ & $\begin{array}{l}\text { Zinc } \\
\text { Iron }\end{array}$ & $\mathrm{Pb}>\mathrm{Fe}>\mathrm{Zn}>\mathrm{Cd}$ & Wingenfelder et al. (2005) \\
\hline Acidic Mine Water & $\begin{array}{c}\text { Turkish } \\
\text { Clinoptilolite }\end{array}$ & $\begin{array}{l}\text { Iron } \\
\text { Zinc }\end{array}$ & $\begin{array}{l}\text { Copper } \\
\text { Manganese }\end{array}$ & $\mathrm{Fe}>\mathrm{Zn}>\mathrm{Cu}>\mathrm{Mn}$ & Motsi et al. (2009) \\
\hline & $\begin{array}{l}\text { Turkish (Aegean) } \\
\text { Clinoptilolite }\end{array}$ & $\begin{array}{l}\text { Lead } \\
\text { Copper } \\
\text { Iron } \\
\end{array}$ & $\begin{array}{l}\text { Nickel } \\
\text { Zinc }\end{array}$ & $\mathrm{Cu}>\mathrm{Zn}>\mathrm{Fe}$ & Kocasoy and Sahin (2007) \\
\hline Actual Industrial & Synthetic Zeolite & $\begin{array}{l}\text { Lead } \\
\text { Copper } \\
\text { Iron } \\
\text { Zinc }\end{array}$ & $\begin{array}{l}\text { Nickel } \\
\text { Arsenic } \\
\text { Chromium }\end{array}$ & $\begin{array}{c}\mathrm{Fe}>\mathrm{As}>\mathrm{Pb}>\mathrm{Zn}> \\
\mathrm{Cu}>\mathrm{Ni}>\mathrm{Cr}\end{array}$ & Rios et al. (2008) \\
\hline Wastewater & Fly Ash Zeolite & $\begin{array}{l}\text { Lead } \\
\text { Copper } \\
\text { Iron } \\
\text { Nickel } \\
\text { Zinc } \\
\end{array}$ & $\begin{array}{l}\text { Barium } \\
\text { Manganese } \\
\text { Strontium } \\
\text { Cadmium } \\
\text { Chromium } \\
\end{array}$ & $\begin{array}{c}\mathrm{Pb}>\mathrm{Cd}>\mathrm{Zn}>\mathrm{Cu} \\
>\mathrm{Fe}>\mathrm{Ni}>\mathrm{Ba}\end{array}$ & Prasad and Mortimer (2011) \\
\hline & $\begin{array}{c}\text { American } \\
\text { Clinoptilolite }\end{array}$ & $\begin{array}{l}\text { Zinc } \\
\text { Copper } \\
\text { Iron }\end{array}$ & $\begin{array}{l}\text { Aluminum } \\
\text { Manganese }\end{array}$ & $\begin{aligned} & \mathrm{Fe}>\mathrm{Al}>\mathrm{Cu} \\
> & \mathrm{Zn}>\mathrm{Mg}>\mathrm{Mn}\end{aligned}$ & $\begin{array}{c}\text { Cui et al. (2006) } \\
\text { Xu et al. (2010, 2012-2014) }\end{array}$ \\
\hline
\end{tabular}


The sorption of HMIs in multi-component systems is considered intricate due to solutesolute competition and solute-surface interactions (Wilson, 1994), and the complexity in the interpretation of these systems are influenced by the $\mathrm{pH}$, HMI ionic radii, electronegativity, as well as active site availability on the sorbent material itself (Mohan and Chander, 2006). In addition, the existence of HMIs in industrial wastewater effluents is site-specific, and the concentrations differ and fluctuate extensively (Wilson, 1994). This is demonstrated in Table 1.3, which provides a summary of diverse industrial wastewater effluents, and evidently vast variations of metal concentrations. Helfferich (1962, p. 201) points out that for multi-component systems, the exchange rate may vary for the various counter-ions of HMIs in solution, with the possibility that the concentrations of certain species in either the sorbent or solution may fluctuate prior to attaining its balanced state. This reiterates that real-life fluctuations of HMIs in industrial wastewater effluents are widespread.

Confounding factors, such as the sorption phenomena, interactions along the migration pathway, or non-seasonal features, have an overriding impact on many industrial wastewater stream characteristics (Wilson, 1994). The Canada-Wide Survey of Acid Mine Drainage (Wilson, 1994) is based on a census of 30 mines from data acquisition of 75 stream records located across Canada. Various mine types, such as copper-zinc and nickel-copper, and waste from different sources that include mine water, tailings pond water, seepage/runoff/streams, tailings effluent, and treatment/collection systems, are reported. Based on metal processing mines at the time of this survey, the metals commonly found in acid mine drainage streams include lead, copper, iron, nickel, zinc, arsenic, cadmium, cobalt, aluminum, and manganese. This survey also reports that copper concentrations tend to be highest in the spring and lowest in the fall (both moderate trends); iron (weak trend) and lead (strong trend) concentrations tend to be highest in the winter; zinc concentrations tend to be highest in the spring (weak trend) and lowest in the summer and fall (strong trend). A moderate trend towards the occurrence of the highest concentration of sulphate occurs in the winter, and a strong trend towards the occurrence of the lowest concentration in the spring (Wilson, 1994). In addition to HMIs, other constituents such as the variations in minerals, micro-organisms, and (weather and seasonal) temperature all influence the quality and quantity of industrial waste streams (Motsi, 2010). A survey by Dinardo et al. (1991) selected acidic mineral effluents in Canada that contain high metal concentrations of copper, lead, zinc, nickel, aluminum, total iron and sulphate. The Canadian 
Minister of Justice Metal Mining Effluent Regulations outline the authorized limits of deleterious substances (Schedule 4) (CMJ, 2014), for arsenic, cyanide, lead, copper, lead, nickel, and zinc. This regulation also highlights the need for effluent water quality monitoring based on the concentrations of aluminum, cadmium, iron, mercury, selenium, ammonia, and nitrate. Lead in particular is considered an acute toxic priority (OHSA, 2014). Due to a greater presence in Ontario mines presented by Wilson (1994), the strict limitations required by the Canadian Government (CMJ, 2014), and most importantly, the metals of persistent toxic concern as emphasized by the Great Lakes Water Quality Agreement 1978 amendment, this research study focuses on the presence of lead $\left(\mathrm{Pb}^{2+}\right)$, copper $\left(\mathrm{Cu}^{2+}\right)$, iron $\left(\mathrm{Fe}^{3+}\right)$, nickel $\left(\mathrm{Ni}^{2+}\right)$ and $z$ inc $\left(\mathrm{Zn}^{2+}\right)$ ions that are commonly found in industrial wastewater effluents.

A majority of previous research on sorption capacity of zeolite has investigated synthetic simple solute solutions spiked in single-component systems, and has demonstrated greater removal compared to investigating actual industrial wastewater effluents (Motsi et al., 2009; Xu et al., 2013). However, there is still a disparity in research that limits the understanding of sorption capacity by zeolite for heavy metals and the associated mechanisms when in various multi-component systems (Inglezakis et al., 2003; 2004). Additional research is needed to address this research gap on HMIs in their simultaneous sorption, and to quantify the uptake interference by zeolite of these HMIs in combination (Borandegi and Nezamzadeh-Ejhieh, 2015); in order to utilize the mineral's potential in tertiary industrial treatment processes (Vaca-Mier, 2001) for technology development.

A well-referenced review by Wang and Peng (2010) highlights the recent developments of natural zeolites as adsorbents in water and wastewater treatment, where the influent characteristics (i.e., synthetic, simulated or actual wastewater) vary substantially. With this in mind, it is important to note that the mining industry reference pertaining to the four chapter manuscripts embodied in the Dissertation document does not preclude the fact that the findings are also transferable to other industries and applications. The synthetic simple HMI solute permitted the analysis in a controlled environment for improved quantification, and identification of the important trends established by the dual-column system prototype; which was the fundamental goal and major contribution of this research project. 
Table 1.3 Metal Concentrations of Various Industrial Wastewater Effluents (mg/L)

\begin{tabular}{|c|c|c|c|c|c|c|c|c|c|}
\hline \multirow[t]{2}{*}{ Metal } & \multirow[t]{2}{*}{$\begin{array}{l}\text { Batter Factory } \\
\text { Waste Sample }^{\text {a }}\end{array}$} & \multicolumn{2}{|c|}{$\begin{array}{c}\text { Electrolytic Copper } \\
\text { Industrial Wastewater } \\
\text { Tank Sample } \\
\end{array}$} & \multirow{2}{*}{$\begin{array}{l}\text { British Columbia } \\
\text { Highway 97C } \\
\text { Acid Rock } \\
\text { Drainage }\end{array}$} & \multirow[t]{2}{*}{$\begin{array}{c}\text { Typical } \\
\text { Gold Mine } \\
\text { Wastewater }^{d}\end{array}$} & \multirow[t]{2}{*}{$\begin{array}{c}\text { Wheal } \\
\text { Jane Mine }\end{array}$} & \multirow[t]{2}{*}{$\begin{array}{l}\text { Electroplating } \\
\text { Rinse Waters }\end{array}$} & \multirow[t]{2}{*}{$\begin{array}{c}\text { Pretreatment } \\
\text { Requirement for } \\
\text { Discharge to POTW }\end{array}$} & \multirow{2}{*}{$\begin{array}{c}\text { Copper } \\
\text { Production } \\
\text { Plant } \\
\text { Scrubber }^{\text {g }}\end{array}$} \\
\hline & & Equalization & Neutralization & & & & & & \\
\hline $\mathrm{pH}$ & $3.8-5.8$ & --- & --- & --- & 7.4 & --- & $5-12$ & $5.5-12$ & 1.81 \\
\hline $\mathrm{Pb}$ & $4.0-13$ & 2.4 & 3.8 & --- & 0.140 & --- & 0.7 & 2 & --- \\
\hline $\mathrm{Cu}$ & $<0.0033-0.38$ & 404 & 1806 & 0.17 & 5.063 & 12 & 3 & 3 & 0.65 \\
\hline $\mathrm{Fe}$ & $0.02-20$ & 42.5 & 206 & 0.82 & 0.114 & 200 & --- & $\begin{array}{ll}-- \\
\end{array}$ & --- \\
\hline $\mathrm{Zn}$ & $0.6-17$ & 49.5 & 307 & 101.2 & 0.042 & 85 & 2 & 5 & 18.4 \\
\hline $\mathrm{Cd}$ & $0.02-0.12$ & --- & --- & --- & --- & 1 & 0.5 & 0.5 & 0.73 \\
\hline As & --- & $\begin{array}{ll}-- \\
\end{array}$ & 1.9 & --- & 7.35 & 9 & --- & 1 & --- \\
\hline $\mathrm{Cr}$ & $<0.0044-0.08$ & --- & --- & --- & --- & --- & 3 & 2.75 & --- \\
\hline $\mathrm{Co}$ & --- & --- & 1.26 & --- & --- & --- & --- & --- & --- \\
\hline $\mathrm{Mn}$ & 0.04-0.6 & --- & 1.3 & 10.7 & --- & 15 & --- & --- & 0.08 \\
\hline $\mathrm{Si}$ & --- & --- & --- & 44.2 & --- & --- & --- & --- & --- \\
\hline $\mathrm{Ca}$ & $83-255$ & 620 & 610 & 204 & --- & --- & --- & --- & 71.7 \\
\hline $\mathrm{Na}$ & --- & 210 & 565 & 181 & --- & --- & --- & --- & 9.2 \\
\hline $\mathrm{K}$ & --- & 3.1 & 6.3 & --- & --- & --- & --- & --- & 1.4 \\
\hline $\mathrm{Mg}$ & $15-26$ & --- & --- & 52.3 & --- & --- & --- & --- & 22.2 \\
\hline $\mathrm{Sb}$ & --- & 1.8 & 4.8 & --- & --- & --- & --- & --- & 0.25 \\
\hline $\mathrm{Sn}$ & --- & --- & 6.6 & --- & --- & --- & --- & --- & --- \\
\hline $\mathrm{Se}$ & --- & --- & --- & --- & --- & --- & --- & --- & 1.27 \\
\hline $\mathrm{B}$ & --- & --- & --- & --- & --- & --- & --- & --- & 0.12 \\
\hline
\end{tabular}

${ }^{\mathrm{a}}$ (Yabe and de Oliveira, 2003); ${ }^{\mathrm{b}}$ (Kocasoy and Sahin, 2007); ${ }^{\mathrm{c}}$ (Cui et al., 2006);

d (Acheampong et al., 2009); ${ }^{\text {e }}$ (Motsi et al., 2009); ${ }^{\mathrm{f}}$ (Benjamin, 2010, p.4); ${ }^{\mathrm{g}}$ (Beyazit, 2013) 


\subsection{Techniques and Challenges of Current Treatment Technology Research}

As emphasized previously, heavy metals must be removed by advanced treatment methods prior to discharge (Stylianou et al., 2007a; Stylianou et al., 2007b; Asubiojo and Ajelabi, 2009; Barakat, 2011; Xu et al., 2012; Nuic et al., 2013; Markovic et al., 2015). The advancement of treatment processes requires the development and optimization of new operations that incorporate low-cost raw materials with favourable removal efficiency (Markovic et al., 2015), with an overall simple, resilient and cost-effective strategy (Vaca-Mier et al., 2001; Margeta et al., 2013). However, the feasibility of migration control becomes a challenge due to the site-tosite variability and complex industrial wastewater effluents (Akcil and Koldas, 2006); in addition to the strict limitations imposed by wastewater discharge water regulations (Bektas and Kara, 2004) of local water recipients (Markovic et al., 2015). This is a reoccurring proclamation by all industry that must address effluents which contain heavy metals.

Innovative treatment technologies are a challenge for all related industries, which include but are not limited to high associated economic costs and pollutant specific methods. Consequently, the conversion of inorganic ion-exchangers into hybrid fibrous or nanoscale ionexchangers is considered to be the latest development of the water treatment industry. These materials are gaining attention, as they demonstrate a high efficiency and rate of sorption with short diffusion path towards environmental pollutants. Among metal-containing nanoparticles, carbonaceous materials and dendrimers, zeolites are considered as one of the most progressive functional and nano-sized materials of the millennium. The prospects of this natural mineral are promising, and its unique position is attributed to its sorption properties particularly through their surface treatment. Nanoscale science and engineering developments are providing extraordinary opportunities to develop more cost effective and environmentally acceptable water purification processes (Chmielewská et al., 2008).

Columns are a convenient approach for industrial-scale treatment applications (Vukojevic Medvidovic et al., 2013), and are frequently implemented in sorption studies such as in Inglezakis et al. (2004), Mohan and Chander (2006), Peric et al. (2009), Aydin and Saygili (2009), and Nuic et al. (2016). The continuous-mode process demonstrates an efficiency in treating large effluent volumes (Nuic et al., 2016), which requires less investment and operational costs, and is more economically feasible than in a batch-mode configuration (Nuic et 
al., 2016) (i.e., single-stirred tanks, series of mixed vessels) (Cui et al., 2006). The general classification of columns or 'multi-phase reactors' consist of three major categories: (1) trickle bed reactors, (2) fluidized bed reactors, and (3) bubble column reactors (Kantarci et al., 2005). The overall performance and practicality of a reactor is influenced by the sorbent material (zeolite), as well as the efficiency of the sorption process (equilibrium and kinetics); all of which depends on the unsteady state process of the space and time distribution relationship to the mass transfer mechanisms between phases (liquid, solid, gas) (Nuic et al., 2016).

As presented in the packed-bed reactor theory by Nuic et al. (2016), the trickle bed reactor is such that the sorbent material particles are fixed and the concentration gradient is constant (the feed solution with the same concentration enters the column continuously); it is also known as a fixed-bed reactor (FBR). The overall sorption process rate is controlled by mass transfer, and is influenced by the concentration profile over time. The transport into and out of the reactor volume are due to the same mechanisms, which include dispersion (a function of turbulence; random fluctuations in the movement of fluid transport of dissolved and suspended matter) and diffusion (random motion of molecules) (Droste, 1997, p. 244). In a packed bed, the sustained axial flow encompasses diffusion-convection interactions; there is no sustained radial flow and therefore radial molecular diffusion and convective dispersion may be considered independent random motions (Gunn, 1987).The mass transfer process depends fundamentally on: (1) convection (flow of solution through the zeolite layer, a pathway for the binding of HMIs onto the zeolite particle surface), and (2) axial dispersion (flow of solution by-passing around the zeolite particle in the column direction). At the point of inflection, the controlling mechanism changes from convection (through the liquid phase) to dispersion (through the zeolite particle itself). As highlighted by Cui et al. (2006), fixed-bed reactors are at an advantage by providing little axial dispersion, operating very similar to a plug flow (PF) hydraulic regime. In a PF regime, the water flows through the reactor in a series of plugs. There is no transfer of contents between plugs; consistent with vertical and lateral mixing (excluding longitudinal) (Droste, 1997, p. 245). A steady state flow situation exists, and there is no mixing between adjacent planes in the reactor (Droste, 1997, p. 258).

A bubble column reactor is essentially a vessel with a gas distributor at the base, such that the gas is sparged into either a liquid or liquid-solid suspension; known as a slurry bubble 
column (SBC) in the presence of a solid phase (zeolite) (Kantarci et al., 2005). The solid phase particles are relatively small in size and are suspended by very vigorous relative motion influenced by the superficial gas velocity (Cui et al., 2006). This reactor category demonstrates excellent heat and (external and internal) mass transfer characteristics (Kantarci et al., 2005; Cui et al., 2006). In comparison to FBR, Cui et al. (2006) demonstrates that a SBC provides a shorter residence time (faster uptake) required for sorption, as well as an ease of sorbent replacement to the column for regeneration.

The process effectiveness of industrial wastewater treatment by zeolite depends on the quality of the clinoptilolite and the initial composition of the effluent (Xu et al., 2014). The critical review of literature has demonstrated the application of numerous natural zeolite deposit sources in the removal technology development of industrial wastewater contaminants such as HMIs; including the United States (Ciosek and Luk, 2017), Mexico (Vaca-Mier, 2001), Serbia (Nuic, Peric, Trgo, Vukojevic Medvidovic, 2006-2016), and Greece (Inglezakis et al., 20012010). Margeta et al. (2013) summarizes the chemical composition of natural zeolites from different deposits, expressed as mass fraction of oxide components; which highlight the complex mineralogical composition depending on the country of origin. Once again, the summary of influent stock and zeolite diversity (clinoptilolite source, content and mineralogical properties/quality) highlights the consistency among HMI removal capacity and selectivity trends; which lends to an additional challenge to the unity of terminology (i.e., capacity), the advancement of source control treatment technology development being a worthwhile pursuit.

With technical feasibility in mind in terms of operation and maintenance, the treatment method of sorption may be considered expensive compared to current treatment methods. However, the revenue generated from the recovered metals may offset the associated costs and offers significant environmental benefits due to the recovered, reusable form of the metals of interest (Dinardo et al., 1991). In addition, to date, many research efforts have employed zeolite in the various multi-phase reactor configurations described above. This prompted the motivation to explore a new treatment system configuration that would provide a different perspective on how to remove the heavy metals, with the potential for future optimization in the removalregeneration-recovery process. 


\section{RESEARCH MOTIVATION AND AUTHORSHIP}

\subsection{Research Objectives and Scope}

To date, significant industrial interests have established numerous research groups for the investigation of the natural mineral zeolite as a sorbent material in treatment technology process development; such groups include but are not limited to Inglezakis et al. (2001-2010), Motsi (2009-2010), Nuic et al. (2013-2016), Peric et al. (2004, 2009), Vukojevic Medvidovic et al. (2006-2013), and Xu et al. (2010, 2012-2014). Based on the trends of the critical review presented in Chapter 1, the following research gaps define the problem statement that is addressed by this research endeavour:

1. The investigation of the zeolite mineral in its natural state, without modification or pretreatment, as a more industry-viable, environmentally-conscious material alternative in the development of sorption treatment technology;

2. The greater understanding of the removal capacity by zeolite for heavy metals and the associated mechanisms and uptake interferences when in various HMI multi-component systems, and;

3. The design of a FBR treatment system to incorporate automation and adaptable modules.

The fundamental goal of this research project is to investigate the zeolite mineral and the sorption process of HMIs that are commonly found in industrial wastewater effluent, with the development of a sorption system prototype as the fundamental contribution. The scope of this research involves the mineral in its natural state and indicates lead $\left(\mathrm{Pb}^{2+}\right)$, copper $\left(\mathrm{Cu}^{2+}\right)$, iron $\left(\mathrm{Fe}^{3+}\right)$, nickel $\left(\mathrm{Ni}^{2+}\right)$ and zinc $\left(\mathrm{Zn}^{2+}\right)$ as the HMIs of interest, to be combined in various (single, dual-, triple-, multi-) component systems. The specific objectives of the study are outlined in Figure 2.1, detailing the four journal manuscripts that pertain to the phases of the research project. 
Table 2.1 Dissertation Chapters - Research Project Phases and Focus

\section{DISSERTATION CHAPTER}

FOCUS

To demonstrate the effects of operation parameters (particle size, sorbent-to-sorbate dosage, influent concentration, contact time, set-temperature, heat pre-treatment) on the sorption process.

To investigate the systematic increase of HMIs in various (single, dual, triple, multi-) component system combinations.

To demonstrate the effects on zeolite selectivity (order) of lead $\left(\mathrm{Pb}^{2+}\right)$ uptake.

To investigate the associated mechanisms and rate of the sorption process in various component system combinations.

To demonstrate the representation of kinetic models; reaction-type and diffusion-type on the uptake of HMIs by zeolite.

To design and construct a packed, fixed-bed, dualcolumn sorption system containing natural zeolite.

7 An Innovative Dual-Column System for Heavy Metallic Ion Sorption by Natural Zeolite
To investigate the performance of the sorption system.

To demonstrate the system treatability (i.e., empty bed contact time, breakthrough curve, usage rate).

The major contributions of this research to the scientific community include:

1. A methodical experimental design to evaluate removal trends in various HMI component system combinations, furthering the understanding of the effects on the sorption capacity of zeolite in its natural state;

2. The kinetic analysis of the sorption rate of the HMIs of interest, that are methodically combined in various component systems;

3. Consistent removal selectivity trends in batch-mode, with the trend validation in continuousmode configuration and supporting literature;

4. The innovative design of a dual-column sorption system prototype, with proven on-trend removal performance, and;

5. Exclusive automated, variable-flow prototype configuration with a custom sampling technique potential; a platform for the further advancement of intelligent process controls. 
The removal of heavy metals and other industrial waste pollutants is regarded as a very complex process. Nevertheless, there is still a great need for continued advances in treatment technology to achieve industry remediation goals and to meet government regulations. The

fundamental contributions of 'proof-of-concept' and the thought-provoking development of technology design in the research endeavour presented in the Dissertation document is certainly a worthwhile pursuit.

\subsection{Dissertation Structure}

This Dissertation is based on a four-phase research project, and is submitted as a manuscriptstyle document, composed of eight chapters and six appendices. A summary of each chapter is presented as follows:

\section{Chapter 1}

A critical review of previous research efforts in the treatment of industrial wastewater of heavy metals with natural zeolite is presented in the introductory chapter, in order to expose existing research gaps and to outline the experimental program.

\section{Chapter 2}

This chapter defines the objectives and scope of the research project, as well as proclaim the major contributions to the scientific community. The document structure as well as the statement of authorship are also provided.

\section{Chapter 3}

This chapter outlines the fundamental aspects to the experimental methodology and quality control protocol that have been implemented in the research; which present the consolidated 'Materials and Methods' for cohesion in the document.

The following four chapters are the manuscripts that discuss the significant findings of each phase of the research project. 


\section{Chapter 4}

The effects of preliminary parameters and operative conditions (particle size, sorbent-to-sorbate dosage, influent concentration, contact time, set-temperature, and heat pre-treatment) are demonstrated.

\section{Chapter 5}

The selectivity of natural zeolite for lead $\left(\mathrm{Pb}^{2+}\right)$ ion in the presence of competitive ions is evaluated.

\section{Chapter 6}

The modelling of removal kinetics using 'reaction-type' and 'diffusion-type' models is discussed.

\section{Chapter 7}

The design and removal performance of a packed, fixed-bed, dual-column sorption system prototype is investigated.

\section{Chapter 8}

This chapter presents a summary of the fundamental conclusions of the research project, and the recommendations for further study in the engineering practice are discussed.

\section{Appendices}

The appendices provide the details of all the experimental procedures and schedule (A), analytical technology operating procedure (B), as well as technical conference papers $(\mathrm{C}, \mathrm{D}, \mathrm{E})$. Appendix F highlights a cover featured journal publication that investigates the capacity of natural zeolite to remove nutrients from wastewater; a former research endeavour investigated by the Ph.D. Candidate. 


\subsection{Statement of Authorship}

Amanda L. Alaica-Ciosek conceived and designed all experiments, with feedback from Dr. Grace K. Luk. Alaica-Ciosek designed and constructed the sorption system prototype, performed all experiments and analytical simulations, as well as analyzed all the data. The journal manuscripts were written by Alaica-Ciosek with input from Luk. The following chapter specifications provide the journal manuscripts and publication details pertaining to this Dissertation document.

\section{Chapter 4}

Effects of Operation Parameters on Heavy Metallic Ion Removal from Mine Waste by Natural Zeolite International Journal of Environmental Pollution and Remediation

Published: 07 February 2018.

doi:10.11159/ijepr.2018.002.

\section{Chapter 5}

Lead Removal from Mine Tailings with Multiple Metallic Ions

International Journal of Water and Wastewater Treatment

Published: 19 January 2017.

doi:10.16966/2381-5299.134.

\section{Chapter 6}

Kinetic Modelling of the Removal of Multiple Heavy Metallic Ions from Mine Waste by Natural Zeolite Sorption

Water - Special Issue - Treatment of Wastewater and Drinking Water through Advanced Technologies

Journal Citation Reports ${ }^{\circledR}$ (Clarivate Analytics) Impact Factor: 1.832 (5-Year: 2.056)

CiteScore (Scopus): 2.05; Rankings: 34/88 (Q2) in 'Water Resources' category.

Published: 01 July 2017.

doi:10.3390/w9070482.

\section{Chapter 7}

An Innovative Dual-Column System for Heavy Metallic Ion Sorption by Natural Zeolite Applied Sciences - Special Issue - Wastewater Treatment and Reuse Technologies

Journal Citation Reports ${ }^{\circledR}$ (Clarivate Analytics) Impact Factor: 1.679 (5-Year: 1.913)

Rankings: 150/275 (Q3) in "Materials Science, Multidisciplinary", 91/166 (Q3) in "Chemistry, Multidisciplinary", and 75/147 (Q3) in "Physics, Applied" categories.

Published: 05 August 2017.

doi:10.3390/app7080795. 


\section{EXPERIMENTAL RESEARCH DESIGN}

The methodology presented in this chapter is the consolidation of the "Materials and Methods' subsections pertaining to the four journal manuscripts presented in Chapters 4 to 7; as identified in the Preface and Chapter 2, in accordance with of the manuscript-style structure of the Dissertation document.

\subsection{Experimental Methodology}

Table 3.1 outlines the fundamental experimental factors (i.e., analytical method, sample size, contact period, cleaning cycle, etc.) that were kept constant throughout the experimental work.

Table 3.1 Established Experimental Factors throughout Analysis

\begin{tabular}{|c|c|c|}
\hline Factor & Description & Reference \\
\hline $\begin{array}{l}\text { Elemental } \\
\text { Analysis }\end{array}$ & $\begin{array}{c}\text { Perkin Elmer } \\
\text { Optima } 7300 \text { ICP-AES } \\
\text { Triplicate Combination }\end{array}$ & $\begin{array}{l}\text { University of Toronto } \\
\text { ANALEST Centre }\end{array}$ \\
\hline $\begin{array}{c}\text { Sorbent } \\
\text { Cleaning Cycle }\end{array}$ & $\begin{array}{l}\text { Sieve Debris - DDW Rinse } \\
\text { Moisture - Dry } 24 \mathrm{hr} \text { at } 80^{\circ} \mathrm{C}\end{array}$ & Inglezakis et al. (2001) \\
\hline Filtration & Syringe via 0.45 um Filter & \\
\hline $\begin{array}{l}\text { Acidification } \\
\text { Preservation }\end{array}$ & $\mathrm{pH}$ Level to 2 via $\mathrm{HNO}_{3}$ acid & Rice et al. (2012) \\
\hline $\begin{array}{l}\text { Agitation } \\
\text { Method* }\end{array}$ & $\begin{array}{c}\text { Thermo-Fischer Scientific } \\
\text { MaxQ } 4450 \text { Orbital Shaker } \\
\text { Triple Eccentric Drive Platform }-400 \mathrm{rpm}\end{array}$ & $\begin{array}{l}\text { Ryerson University } \\
\text { Environmental } \\
\text { Engineering Lab }\end{array}$ \\
\hline $\begin{array}{c}\text { Contact } \\
\text { Time (min)* }\end{array}$ & $\langle 5,10,15,30,45,60,90,120,180\rangle$ & $\begin{array}{c}\text { Motsi et al. (2009) } \\
\text { Sprynskyy et al. (2006) }\end{array}$ \\
\hline $\begin{array}{c}\text { Sample } \\
\text { Volume }(\mathrm{mL}) *\end{array}$ & 100 & $\begin{array}{c}\text { Ouki and Kavannagh (1999) } \\
\text { Motsi et al. (2009) }\end{array}$ \\
\hline
\end{tabular}

The various experimental procedures are provided in Appendix A. The following subsections elaborate on the zeolite mineral particle size and cleaning cycle, influent stock preparations, as well as elemental analysis selection and sorption capacity calculations. 


\subsubsection{Sorbent Material}

This study employed a natural zeolite mineral sample composed primarily of $85-95 \%$ clinoptilolite (CAS No. 12173-10-3) with traces of opaline silica, and is sourced from a deposit located in Preston, Idaho (Bear River Zeolites, 2012; 2017). The natural zeolite sample specifications are provided in Table A.2. Typical elemental analysis indicated the presence of various elements, including sodium, calcium, magnesium, and potassium, as well as lead, copper, iron, and zinc. No significant concentrations of toxic trace elements were present in its composition, nor are trace metal elements water soluble. The low-clay content unique to this sample ensured good hydraulic conductivity, low dust content, and a harder and more resistant structure (Bear River Zeolites, 2012; 2017). Figure 3.1 is a view of the natural zeolite mineral sample employed in this research, as presented in the Dissertation document.
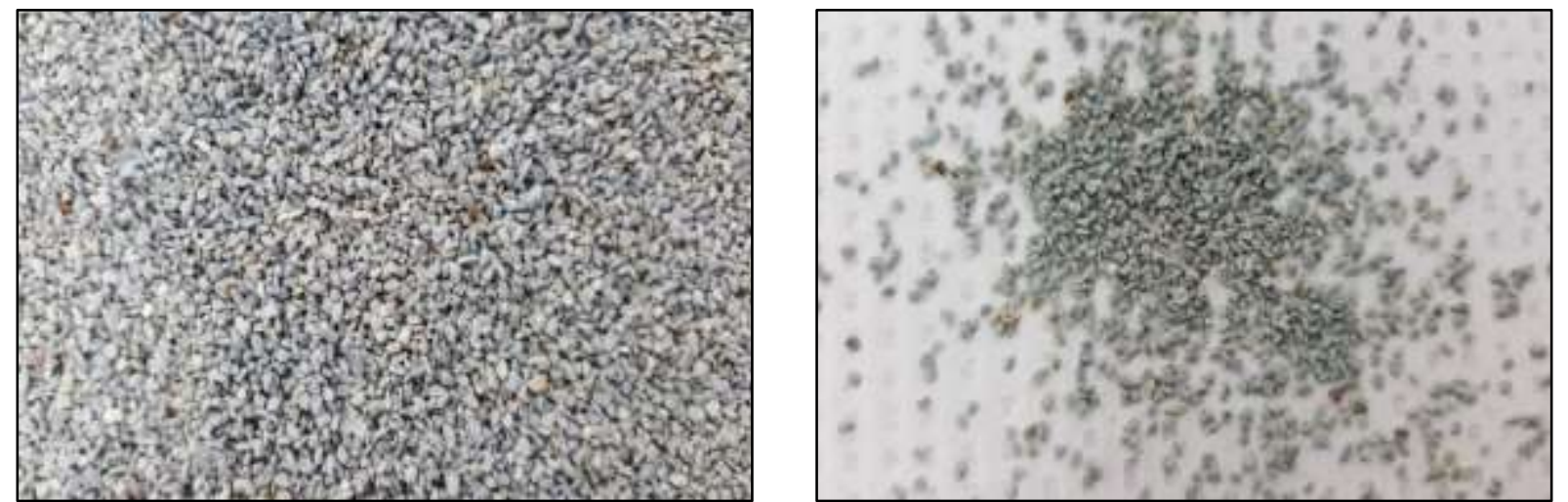

Figure 3.1 Granular View of Natural Zeolite Sample

Preliminary analysis of the raw zeolite mineral supply involved a particle size range within the standard mesh fractions of $-8+40,-14+40$, and $-30+60$; discussed with greater detail in Appendix A.6. The particle size range from $1.41 \mathrm{~mm}$ (pass No. 14) to $0.420 \mathrm{~mm}$ (retain No. 40) is divided into sizes $A\left(d_{p, A}\right)(1.190-1.410 \mathrm{~mm}), B\left(d_{p, B}\right)(0.707-0.841 \mathrm{~mm}), C\left(d_{p, C}\right)(0.420$ $0.595 \mathrm{~mm})$ and $\mathrm{D}\left(\mathrm{d}_{\mathrm{p}, \mathrm{D}}\right)(0.841-1.19 \mathrm{~mm})$; with standard mesh sieves and a mechanical shaker. Overall, these four divisions were selected to provide a distinct variance, based on the approximate distribution of the $+14-40$ material source, as displayed in Table 3.2. 
Table 3.2 Preliminary Distribution of $+14-40$ Zeolite Supply

\begin{tabular}{|c|c|c|c|c|}
\hline & \multicolumn{2}{|c|}{ Test Sample Size (g) } & \multicolumn{2}{|c|}{1006.60} \\
\hline & \multicolumn{2}{|c|}{ Sieve Gradations } & \multicolumn{2}{|c|}{$\begin{array}{c}\text { Sample } \\
\text { Distribution }\end{array}$} \\
\hline & & & (g) & $(\%)$ \\
\hline & & \#14 Retain & 76.5 & 7.60 \\
\hline $\mathbf{A}$ & \#14 Pass & \#16 Retain & 200 & 19.9 \\
\hline \multirow{2}{*}{ D } & \#16 Pass & \#18 Retain & 181 & 18.0 \\
\hline & \#18 Pass & \#20 Retain & 151 & 15.0 \\
\hline \multirow[t]{2}{*}{ B } & \#20 Pass & \#25 Retain & 119 & 11.8 \\
\hline & \#25 Pass & \#30 Retain & 94.0 & 9.34 \\
\hline \multirow{2}{*}{$\mathbf{C}$} & \#30 Pass & \#35 Retain & 68.1 & 6.77 \\
\hline & \#35 Pass & \#40 Retain & 48.3 & 4.80 \\
\hline \multicolumn{3}{|c|}{ \#40 Pass (PAN) } & 57.9 & 5.75 \\
\hline \multicolumn{3}{|c|}{ SUM } & 996.0 & 98.93 \\
\hline \multicolumn{3}{|r|}{ LOST } & 10.8 & 1.07 \\
\hline
\end{tabular}

The particle size operation parameter is investigated in Chapter 4 of the Dissertation document. All subsequent phases of the research employed the $d_{p, D}$ size exclusively, with a geometric mean diameter of $1.00 \mathrm{~mm}$ (Mullin, 2001); which demonstrated the greatest percent yield within the +14-40 source and also, being a broader, coarser size range, is of interest to this study; being a more conservative, viable range. As suggested by the laboratory-scale packed bed system investigations by Inglezakis et al. (2001b), a zeolite fraction of $0.8 \mathrm{~mm}$ to $1 \mathrm{~mm}$ nominal diameter is recommended for the continuous-mode (columns) to ensure the full exploitation of the material but also to prevent considerable pressure drop during the analysis period.

Low-cost and readily available raw materials without any further economic investment (i.e., pre-treatment) are very useful for any industrial use (Markovic et al., 2015). Therefore, the zeolite mineral sample was applied in its natural state, without any chemical modifications, to minimize all associated costs and environmental impacts of this study. The sieved zeolite was exposed to a cleaning cycle, which involved rinsing in deionized distilled water to remove debris and dust, and drying at $80 \pm 3^{\circ} \mathrm{C}$ (Isotemp® Oven Model 630G; Fisher Scientific, USA) for 24 hours to remove any residual moisture (Inglezakis et al., 2001a). 


\subsubsection{Sorbate Solution}

\subsubsection{Heavy Metallic Ion Concentration}

Heavy metals are elements with atomic weights between 63.5 to 200.6, and a specific gravity greater than 5.0 (Inglezakis and Poulopoulos, 2006, p. 24; Fu and Wang, 2011; Tchounwou et al., 2012). They are classified as either: (1) toxic (i.e., $\mathrm{Pb}, \mathrm{Cu}, \mathrm{Ni}, \mathrm{Zn}, \mathrm{Cd}, \mathrm{Cr}, \mathrm{Co}$, As, $\mathrm{Hg}, \mathrm{Sn}$ ), (2) precious (i.e., $\mathrm{Pd}, \mathrm{Pt}, \mathrm{Ag}, \mathrm{Au}, \mathrm{Ru}$ ), or (3) radionuclides (i.e., U, Th, Ra, Am) (Acheampong et al., 2009). Total metals found in industrial wastewater effluents are composed of both dissolved and suspended fractions, defined as dissolved (unacidified sample passing a $0.45 \mu \mathrm{m}$ filter), suspended (unacidified sample retained by a $0.45 \mu \mathrm{m}$ filter), and total metals (unfiltered sample defined by digestion (dissolved and suspended) (Rice et al., 2012). This research focused on lead $\left(\mathrm{Pb}^{2+}\right)$, copper $\left(\mathrm{Cu}^{2+}\right)$, iron $\left(\mathrm{Fe}^{3+}\right)$, nickel $\left(\mathrm{Ni}^{2+}\right)$ and zinc $\left(\mathrm{Zn}^{2+}\right)$ metals in dissolved fraction, known in their ionic form as HMIs.

The simple synthetic HMI solutions were prepared from analytical grade nitrate salts in deionized distilled water, namely $\mathrm{Pb}\left(\mathrm{NO}_{3}\right)_{2}, \mathrm{Cu}\left(\mathrm{NO}_{3}\right)_{2} \cdot 3 \mathrm{H}_{2} \mathrm{O}, \mathrm{Fe}\left(\mathrm{NO}_{3}\right)_{3} \cdot 9 \mathrm{H}_{2} \mathrm{O}, \mathrm{Ni}\left(\mathrm{NO}_{3}\right)_{2} \cdot 6 \mathrm{H}_{2} \mathrm{O}$, and $\mathrm{Zn}\left(\mathrm{NO}_{3}\right)_{2} \cdot 6 \mathrm{H}_{2} \mathrm{O}$, respectively, and combined equally to maintain a total normality of $0.01 \mathrm{~N}$ (10 meq/L) (Inglezakis et al., 2002, 2003; Kocasoy and Sahin, 2007); charge equivalents (Benjamin, 2010, p. 13). The $\mathrm{NO}_{3}{ }^{-}$anions in the aqueous solution do not influence the ionexchange process, since they do not form any metal-anion complexes and do not hydrolyze in solution (Peric, 2004; Minceva et al., 2008). The corresponding HMI concentrations are approximately $1036 \mathrm{mg} / \mathrm{L}$ for $\mathrm{Pb}^{2+}, 318 \mathrm{mg} / \mathrm{L}$ for $\mathrm{Cu}^{2+}, 186 \mathrm{mg} / \mathrm{L}$ for $\mathrm{Fe}^{3+}$, for $293 \mathrm{mg} / \mathrm{L} \mathrm{Ni}^{2+}$, and $327 \mathrm{mg} / \mathrm{L}$ for $\mathrm{Zn}^{2+}$; discussed with greater detail in Appendix A.5 with supporting calculations in Table A.2. It is important to note that this study methodically increased the HMI combinations to develop a more comprehensive understanding of how the presence of multiple HMIs influences the sorption process, as follows:

- single-component system - 10 meq/L per metal, (lead $[\mathrm{P}]$, copper [C], iron [F], nickel [N], zinc [Z]);

- dual-component system [D] - $5.0 \mathrm{meq} / \mathrm{L}$ per metal (lead-copper [D-PC], lead-iron [D-PF], lead-nickel [D-PN], lead-zinc [D-PZ], copper-iron [D-CF], copper-nickel [D-CN], iron-zinc [D-FZ], nickel-zinc [D-NZ]);

- triple-component system [T] - 3.3 meq/L per metal (lead-copper-iron), and;

- multi-component system $[\mathrm{M}]-2.0 \mathrm{meq} / \mathrm{L}$ per metal (all five metals). 
To examine the effect of competitive HMIs in solution, the appropriate mass of its nitrate salt was dissolved to produce a competitive cation concentration of $0.01 \mathrm{~N}$; with the concentration ratio (CR) aimed to 1 . The $\mathrm{CR}$ refers to the ratio of competitive cation concentration to metal concentration, all in the eq/L scale. As ion-exchange is a stoichiometric process, the effect of cations should be investigated under equal normality $(\mathrm{CR}=1)$ for all metals and cations (Inglezakis et al., 2005). The removal efficiency order is indicative of how the adsorptive nature of zeolite for each ion varies among the different component systems (Inglezakis et al., 2003). Overall, this selectivity or preference of zeolite for one cation compared to another (at given equilibrium conditions and composition) (Bekkum et al., 1991) is stronger for the counter-ion of higher valence, increasing with dilution of solution and strongest with ion-exchange of high internal molality. The counter-ions' valences affect the 'electro-selectivity' of zeolite. The preference is also stronger for smaller solvated equivalent volumes, and forms stronger ionic bonds with fixed ionic groups (Helfferich, 1962). The cations present in the sorbent have valences that differ from those in solution. Consequently, as the dilution increases, the selectivity of the sorbent for the ion with a higher valence also increases. Accordingly, comparative analysis of various metal ions should be conducted at the same normality and temperature, in order to minimize the changes observed in isotherm configuration with dilution (Inglezakis et al., 2003).

As discussed in Chapter 1, the concentrations of HMIs in industrial wastewater effluents are complex and diverse. With that being said, the five HMIs of interest are combined to a total 10 meq/L aqueous solution concentration to maintain an efficient, conservative structure and controlled environment, in order to draw meaningful, quantifiable analysis to this research endeavour presented in the Dissertation document.

\subsubsection{2. pH Levels}

It is important to note that the $\mathrm{pH}$ range under which sorption takes place should be specified (Styianou et al., 2007b) and attentively monitored. The removal of multiple HMIs from aqueous solutions by the sorption of natural zeolite is a complex process consisting of predominately adsorption and ion-exchange. However, at a high initial $\mathrm{pH}$, this process could be accompanied by precipitation and the metal ion hydroxo-complexes formed can be sorbed on zeolite surface sites that encompass different sorption affinity (Peric, 2004). Research has demonstrated (Ouki and Kavannagh, 1999; Ersoy and Celik, 2002; Inglezakis et al., 2003) that 
the sorbate solution $\mathrm{pH}$ affects the uptake of metals, and this is particularly the case for the HMIs that have low preference by zeolite. The uptake mechanism shifts from ion-exchange/adsorption in the acidic region to adsorption/complexation and possible precipitation in the basic region (Minceva et al., 2008). Influential factors include the metal ion speciation and natural stability, as well as the electro-kinetic properties of zeolite in aqueous solutions. At a low $\mathrm{pH}$ level, the hydrogen cation $\left(\mathrm{H}^{+}\right)$is considered as a competitive ion to the HMI during the ion-exchange process (Inglezakis et al., 2003). Decationization (exchange of co-cations with $\mathrm{H}^{+}$ions) and dealumination of the zeolite structure may occur under acidic conditions (Xu et al., 2014). Evidently, the adsorption/ion-exchange process is preferred at higher $\mathrm{pH}$ levels, which should be less than the minimum $\mathrm{pH}$ of precipitation (Styianou et al., 2007a) of the respective metal ions (Mohan and Chander, 2006).

The process of adsorption from the sorbate to the surface of the zeolite mineral is followed by ion-exchange, then hydrolysis; which is detected by the $\mathrm{pH}$ level and electric conductivity of the sorbent-sorbate solution (Margeta et al., 2013). The $\mathrm{pH}$ of the effluent solution decreases, depending on the metal removed (Styianou et al., 2007b); as a function of metal concentration, likely due to two equilibria: (1) ion-exchange, and (2) water hydrolysis (Mohan and Chander, 2006). The two-way reversible relationship between the hydrolysis and hydration of metals is presented in Equation 3.1, which is affected by the acidity of the solution (Inglezakis et al., 2003). At a lower $\mathrm{pH}$, the reaction is shifted to the left and more highly charged metal complexes are formed (Inglezakis et al., 2003), with inorganic ligands such as $\mathrm{OH}^{-}$(Ouki and Kavannagh, 1999); which encourages the sorption processes.

$$
\left[\mathrm{M}\left(\mathrm{H}_{2} \mathrm{O}\right)\right]^{\mathrm{n}+}+\mathrm{H}_{2} \mathrm{O} \rightleftharpoons\left[\mathrm{M}\left(\mathrm{H}_{2} \mathrm{O}\right)_{\mathrm{x}-1}(\mathrm{OH})\right]^{(\mathrm{n}-1)+}+\mathrm{H}_{3} \mathrm{O}^{+}
$$

However, this condition is not favourable as the hydrogen cation is considered as a competitive ion to the targeted HMIs in solution during the sorption process, adversely affecting the overall uptake (Inglezakis et al., 2003). The degree of metal complex formation depends on the $\mathrm{pH}$ level as well as the ionic composition and HMI of interest. Accordingly, the metal speciation influences the removal efficiency of the sorbent and the preference for a given metal is affected by the metal complex characteristics (Inglezakis et al., 2003) that predominate at a given $\mathrm{pH}$ level (Ouki and Kavannagh, 1999). Since the hydrolyzed cations (co-ions) within in the voids and channels are connected by relatively weak electrostatic bonds to the aluminium-silicate 
lattice framework of the zeolite, these co-ions are mobile to exchange with the cations (counterions) from the sorbate. The relative size and charge of cations (co-ions, counter-ions), solution cation concentration and ionic strength, temperature and $\mathrm{pH}$ level, solvent properties as well as zeolite characteristics all influence the quantity of ions and inevitably the rate of exchange (Margeta et al., 2013).

The natural acidity (controlled only by the hydrolysis of metals and not by the addition of acid) of the respective metal solution and stability constants of metal complexes are significant (Inglezakis et al., 2003). The acidity effects on the uptake of $\mathrm{Pb}^{2+}, \mathrm{Cu}^{2+}, \mathrm{Fe}^{3+}, \mathrm{Cr}^{3+}$ investigated by Inglezakis et al. (2003) reports the natural acidities as 4, 3, and 2 for that of $\mathrm{Pb}^{2+}, \mathrm{Cu}^{2+}$, and $\mathrm{Fe}^{3+}$, respectively, and determined that the $\mathrm{Pb}^{2+}$ ion uptake in particular remained stable in a $\mathrm{pH}$ range of 2 to 4; which is an indication of the high preferential level by zeolite. The kinetic study conducted by Kocaba et al. (2007) investigated a pH range of 1 to 10 on the removal of $\mathrm{Cd}^{2+}$, $\mathrm{Cu}^{2+}$, and $\mathrm{Ni}^{2+}$. The sorption percentages were increased sharply after a $\mathrm{pH}$ of 4 for all HMIs. The sorption of $\mathrm{Ni}^{2+}$ remains almost constant up to a $\mathrm{pH}$ of 2, and increased sharply beyond this level. The uptake of $\mathrm{Ni}^{2+}$ reached $86.5 \%$ at a $\mathrm{pH}$ of 6 , to a maximum uptake of $91.9 \%$ at a $\mathrm{pH}$ of 10. The sorption of $\mathrm{Cu}^{2+}$ increased sharply after a $\mathrm{pH}$ of 4 to reach $75.4 \%$ uptake, then increased gradually to $88.9 \%$ at a pH of 6 then $97.6 \%$ at a pH 10. The $\mathrm{pH}$ of 6 was chosen by Kocaba et al. (2007) as the optimum studying $\mathrm{pH}$ to avoid precipitation of HMIs. Generally, it was observed that sorption of HMIs increased by increasing the $\mathrm{pH}$ value. Most of the HMIs tend to form precipites at $\mathrm{pH}$ higher than 6 , which limits this process greatly. Bektas and Kara (2004) reported that the removal of lead ions by clinoptilolite occurs by ion-exchange and physical adsorption when the $\mathrm{pH}$ level is below 6, and the precipitation of $\mathrm{Pb}^{2+}$ ions in the form of $\mathrm{Pb}(\mathrm{OH})_{2}$ increases above this $\mathrm{pH}$ level. Therefore, the sorption of HMIs by zeolite is challenging to quantify at a $\mathrm{pH}$ level greater than 6 , and the actual sorption could be masked by precipitation (Kocaba et al., 2007).

Natural zeolites are known to raise the $\mathrm{pH}$ in acidic aqueous solutions, due to: (1) the ionexchange of $\mathrm{H}^{+}$ions, (2) the binding of $\mathrm{H}^{+}$ions to the Lewis basic sites linked to the oxygen atoms in the zeolite framework, and (3) the $\mathrm{OH}^{-}$ions in solution deriving from hydrolysis of some species present in the zeolite (Stylianou et al., 2007a). The $\mathrm{pH}$ level of the aqueous solution controls the overall sorption process; adsorption of the HMI at the solid-water interfaces as well 
as the ion-exchange of cations within the zeolite structure. Stylianou et al. (2007b) points out that for all minerals, a decrease in the ion-exchange capacity of HMIs occurs for a pH range of 1 to 2. However, very low pH levels may positively influence the sorption process with the hydrolysis of the HMIs in solution (Stylianou et al., 2007a). Also, the $\mathrm{pH}$ level may influence the ionization degree of the sorbate (HMI solution) and the surface property of the sorbent (zeolite mineral) (Minceva et al., 2008). The structural stability of the sorbent should not be compromised; for once the $\mathrm{pH}$ reaches below 1 , the structure of clinoptilolite breaks down in a process termed 'dealumination'. However, zeolites exhibit good structural stability, even in acidic conditions (Motsi, 2010).

Research conducted by Ersoy and Celik (2002) investigated the zeta potential of clinoptilolite, and demonstrates its electro-kinetic properties (i.e., isoelectric point, potential determining point) in aqueous solutions, which are critical to the understanding of adsorption mechanisms of the solid-solution interface. The CEC of clinoptilolite is largely due to the imbalance of charges within the mineral framework. This imbalance is caused by the isomorphous substitutions of $\mathrm{Al}^{3+}$ for $\mathrm{Si}^{4+}$ in the tetrahedral lattice framework (Oter and Akcay, 2007), which enables some exchangeable co-cations, such as the hydrogen ion, to account for the surface charge. These exchangeable co-cations are able to enter into the channels to compensate the positive charge deficiencies (negative charge) of the mineral lattice. Consequently, the $\mathrm{pH}$ level of the system is predicted to affect the sorption behaviour. The potential determining ions were verified to be $\mathrm{H}^{+}$and $\mathrm{OH}^{-}$for the cations of interest. The isoelectric point refers to when the sum of all interactions (i.e., $\mathrm{H}^{+}$and $\mathrm{OH}^{-}$adsorption, dissolved lattice ions distribution, etc.) occurring at the mineral-water interface has a net zero charge. Ersoy and Celik (2002) demonstrated that at a $\mathrm{pH}$ level of 2 , the zeta potential of the clinoptilolite surface is approximately $-18 \mathrm{mV}$; showing a permanent net negative charge at very acidic conditions, which provides an advantage during electrostatic interactions of inorganic cationic contaminants in wastewater.

At lower pH levels, the HMI is more soluble which favours its removal (Xu et al., 2012). Evidently, the initial $\mathrm{pH}$ of the solution must be selected attentively; the aim is to avoid precipitation, for once precipitated, the ions of interest cannot be sorbed (Inglezakis et al.; 2003). Based on the Mine Environment Neutral Drainage (MEND) Program of Canada, typical 
wastewater $\mathrm{pH}$ levels range from 1.8 to 3.9 in the study of acidic mineral effluents and metals (Dinardo et al., 1991), and a range of 2 to 5 based on the census of the Canada-Wide Survey of Acid Mine Drainage Characteristics (Wilson, 1994). Yabe and de Oliveira (2003) reported a pH range of 3.8 to 5.8 from battery processing effluent, while electroplating rinse waters exhibit a range of 5 to 12 (Benjamin, 2010, p. 4). Evidently, depending on the industrial process, the pH of industrial wastewater effluents vary. Hence, this research project was conducted in a conservative manner, such that all pH levels documented were on the acidic end for a controlled experimental program in the analysis of the HMI sorption capacity trends.

The $\mathrm{pH}$ level of every sample (stock solution and sorbent-solution contact) was attentively measured for all operation parameters investigated, using the accumet Basic AB15 pH Meter (Fisher Scientific; CAT No. 13636 AB15). The measurements were conducted before and after contact, and before dilution (following 24 hour storage) preparation for calibration. The influent stock is acidified via commercially high-purity nitric $\left(\mathrm{HNO}_{3}\right)$ acid $\left(3 \mathrm{~mL} 1+1 \mathrm{HNO}_{3} / \mathrm{L}\right.$ sample $)$ (CAS No. 7697-37-2) to a pH of below 2 (Rice et al., 2012); to prevent precipitation of the metal ions (Wang et al., 2009; Inglezakis et al., 2003). Additional trials verified that the filtered and unfiltered HMI influent stock concentrations are the same, indicating both effective dilution practices and complete solubility. Therefore, the concentration of each HMI before and after sorption were exclusively in soluble form. All samples were stored at approximately $4^{\circ} \mathrm{C}$ to prevent volume change due to evaporation; which can be kept stable at ppm levels for up to 6 months (Rice at al., 2012); consistently prepared for all HMI component system combinations.

\subsubsection{Analytical Methodology}

The analytical detection method of the HMI concentration in samples prior to and after zeolite treatment is crucial to the success of the research performed in this project.

The selection of a preferred analytical method is based on solution chemistry (i.e., potential interferences, site-specific samples) and its efficiency (i.e., re-productivity, time and cost). This complexity is attributed to various factors, such as continuous reliability, solution recycling, routine monitoring, minimal by-products, operation of site-specific climatic conditions, solution chemistry, and discharge requirements (Kuyucak et al., 2013). The analytical method selected is determined by the relevance of the method to Canadian standards (Standard Methods for the Examinatoin of Water and Wastewater, $22^{\text {nd }}$ Edition; Rice et al., 2012), the ease and speed of 
detection, the material and equipment costs, and the expected presence of any interfering chemicals.

Analytical methods are categorized as colorimetric and instrumental, which include atomic absorption (AA) spectrometry (flame, electro-thermal, hydride, cold-vapour), flame photometry, inductively coupled plasma (ICP) (atomic emission, mass), and anodic stripping voltammetry (Rice et al., 2012). Among the various atomic spectrometry techniques (i.e., Flame AA, Graphite Furnace AA, ICP-MS), ICP Atomic Emission Spectroscopy (ICP-AES) allows the complete atomization of the elements in a given sample, which minimizes the potential for chemical interferences. It is considered as a true multi-element technique with exceptional sample throughput, and with a very wide range of analytical signal intensity (Perkin Elmer Inc., 2011).

Plasma emission spectroscopy offers many advantages, including lower vulnerability to chemical interference (high excitation temperatures), as well as strong emission spectra outcomes for most elements (which provides multi-elemental analysis with simultaneous recording). This method also provides detectable concentration ranges within several orders of magnitude. With the many emission lines that are associated with this source, this is an advantage for qualitative information but does pose a risk for spectral interferences during quantitative analysis (Skoog et al., 2007).

The plasma consists of an electrically conductive cation-electron based gaseous mixture. Argon gas is primarily used, where the ions absorb sufficient power from the external source to maintain extreme temperatures (of up to $10,000^{\circ} \mathrm{K}$ ), where further ionization indefinitely sustains the plasma state. The ICP is one of three primary types of high-temperature plasmas. Its external source is generated by a torch, which is comprised of three concentric quartz tubes where the argon gas flows. The argon gas consumption occurs at a rate between 5-20 L/min, which varies with the torch design. A water-cooled induction coil surrounds at the top of the largest tube, which is powered by a radio-frequency generator oscillating at 27.12 MHz (Skoog et al., 2007; Rice et al., 2012). The flowing argon is ionized by a spark from a Tesla coil, where the created ions and associated electrons interact with the variable magnetic field created by this coil. The ion-electron interaction causes a flow in the close annular paths, and their resistance cause ohmic heating of the plasma. The heat generated requires thermal isolation of the outer quartz cylinder, which is achieved by the argon flowing tangentially around the tube walls. The torch design is 
viewed by both radial and axial geometry. The sample is introduced to the ICP unit through a concentric glass nebulizer and spray chamber, in the form of an aerosol by the argon flowing at approximately $1 \mathrm{~L} / \mathrm{min}$ (Skoog et al., 2007; Rice et al., 2012).

ICP-AES is applicable to a wide array of elements, which vary in their corresponding detection limits and wavelength lines along the emission spectrum; and is faced with interferences in both the spectral (light emission from spectral sources affect net signal intensity) and non-spectral form (Skoog et al., 2007). Chemical interferences and matrix effects are minimal, but background emissions at low analyte concentrations become an issue when the argon ions significantly recombine with electrons. This issue is addressed by either applicable software auto-corrections or by the operator. Given that the ICP spectrum is abundant for many elements, spectral interferences may arise. Along with a strong spectral line database, current software provides the means to address these issues through wavelength and concentration calibration, spectral analysis, as well as deconvolution of overlapping lines (Skoog et al., 2007) (i.e., data reprocessing).

Based on the single- and multi-component systems of the HMIs selected for this research endeavour, ICP was implemented as the method for elemental analysis (Rice et al., 2012). Mr. Dan Mathers was contacted during experimental design development; who at the time of experimental development and analysis was the Supervisor of the Analytical Lab for Environmental Science Research and Training (ANALEST) (Department of Chemistry University of Toronto, Canada). The ANALEST centre offers the most current technology with their ICP-AES equipment (Optima 7300 DV, Part No. N0770796, Serial No. 077C8071802, Firmware Version 1.0.1.0079; Perkin Elmer Inc.; Waltham, MA, USA), providing rapid, simultaneous measurement of 5,000 emission lines and dual (axial and radial) views, with corresponding WinLab32 Software (Version 4.0.0.0305). The facility provides an ad-hoc training protocol that is instrument specific, which is required for potential users to access the equipment. Self-directed learning computer modules for the application of interest were completed in December 2014 by the Ph.D. Candidate, which was followed by hands-on equipment monitoring and training (3-hours) by ANALEST staff in January 2015. The Standard Operating Procedure (SOP) compiled for the ICP-AES and WinLab32 Software during this hands-on training is presented in Appendix B. 
The analyte primary wavelengths of each HMI element targeted are $327.393(\mathrm{Cu}), 238.204$ $(\mathrm{Fe}), 231.604(\mathrm{Ni}), 220.353(\mathrm{~Pb})$, and $206.200(\mathrm{Zn})$, respectively; with Quantifiable Detection Limits (QDL) corresponding to $0.05 \mathrm{ug} / \mathrm{mL}$ for $\mathrm{Cu}, \mathrm{Fe}, \mathrm{Ni}$, and $\mathrm{Zn}$, and $0.10 \mathrm{ug} / \mathrm{mL}$ for $\mathrm{Pb}$, respectively (per Dan Mathers, ANALEST, 2015). As a measure of electromagnetic radiation (light photons) emission, the light intensity signal is proportional to the specific element analyte wavelength concentration based on calibration. These wavelengths were selected on the basis that they have the strongest emission and provide the best detection limits.

Analysis was conducted with a plasma setting in radial view (to concentrations of greater than $1 \mathrm{mg} / \mathrm{L}$ ), auto sampling of 45 seconds normal time at a rate of $1.5 \mathrm{~mL} / \mathrm{min}$, and a processing setting of 3 to 5 points per peak with 2 point spectral corrections. The calibration curve was generated by applying a stock blank and a multi-element Quality Control Standard 4 with 1, 10, 50, 90, and $100 \mathrm{mg} / \mathrm{L}$ concentrations; as per Standard Methods Part 3000 (Rice et al., 2012). Calibration was conducted through 'linear calculated intercept', and verified 'through zero' that a majority of the trace ions detected are within acceptable QDL levels. Triplicate readings of the HMI aqueous solution and their mean concentrations in calibration units are generated in $\mathrm{mg} / \mathrm{L}$ by the corresponding WinLab32 Software. The sorbed amount of HMI was calculated from the difference between the starting concentration and its concentration in the $0.45 \mu \mathrm{m}$ syringefiltered samples' supernatant. 
The sorption capacity is a significant parameter in the batch-mode analyses and kinetic modelling process. Based on the zeolite mass to a $100 \mathrm{~mL}$ HMI solution volume, the sorption uptake is calculated by the mass balance expressed by Equation 3.2 (Inglezakis et al., 2003; Bektas and Kara, 2004; Erdem et al., 2004; Sprynskyy et al., 2006; Gunay et al., 2007; Motsi et al., 2009; Zolgharnein and Shahmoradi, 2010; Beyazit, 2013; Farouq and Yousef, 2015); applied to the capacity calculations of Chapters 4 to 6 :

$$
\mathrm{q}_{\mathrm{t}}=\frac{\mathrm{V} \times\left(\mathrm{C}_{\mathrm{O}}-\mathrm{C}_{\mathrm{t}}\right)}{\mathrm{M}}
$$

where $\mathrm{q}_{\mathrm{t}}$ (in meq/g) is the sorbed uptake of HMI at time $\mathrm{t}$ (in min), $\mathrm{C}_{\mathrm{O}}$ and $\mathrm{C}_{\mathrm{t}}$ are the HMI concentrations in solution (in meq/L) initially and after time $\mathrm{t}$, respectively, $\mathrm{V}$ is the solution volume (in L), and $\mathrm{M}$ is the zeolite mass (in $\mathrm{g}$ ).

Qualitative analysis of the natural zeolite was conducted to observe the surface morphology over time, by a high-resolution Scanning Electron Microscopy (SEM) technology (6380LV, JEOL, USA), equipped with Oxford energy dispersive X-ray spectroscopy (EDS) and electron backscatter diffraction (EBSD) capacity; accessed at the Department of Mechanical Engineering of Ryerson University. These images are evaluated in Chapter 4 and 5 of the Dissertation document. It is important to note that this study focused on the removal of HMIs from solution, of which the variations in concentrations were detected by the ICP-AES equipment as described in this chapter. For analysis of composition, surface properties and minerology, the use of timeof-flight-secondary-ion mass-spectroscopy (TOF-SIMS), X-Ray Fluorescence (XRF), and XRay Diffraction (XRD) are required. Future research on the optimization of the treatment system prototype should incorporate these additional qualitative and quantitative analytical techniques. 


\subsection{Quality Control (QC) Protocol}

Quality control is of the utmost importance to any and every aspect of research and development. This section to the Dissertation document will point out the substantial efforts made and significant clarifications.

\subsubsection{Experimental Sampling and Statistical Considerations}

The US EPA document (US EPA QA/G-5S, 2002) established a thorough guide in selecting a sampling design protocol. Sampling design (i.e., protocol, handling, quality assurance) is an essential aspect of data collection for the scientific development of decision-making. The key objective is to ensure that the data collected is sufficient to draw overall conclusions about the contamination level in the environment; accurately representing the population by the sample(s). Considerations include the appropriateness, accuracy, and quality (i.e., sample collection, handling methods, and laboratory analysis), effect of measurement error, as well as data representativeness to the study objectives.

There are two sampling design techniques: 1) non-probabilistic (judgmental), and 2) probabilistic. For the purposes of this research, the protocol reflects the latter. It consists of the following sampling forms: simple random, stratified, systematic and grid, ranked set, adaptive cluster, and composite. A 'Data Quality Objectives Process' is a systematic planning approach, with seven key steps:

1. State the Problem

2. Identify the Decision

3. Identify the Inputs
4. Define the Study Boundaries

5. Develop a Decision Rule

6. Specify Tolerable Decision Error Limits

7. Optimize Design for Data Acquirement

In order to determine the ideal sampling design, various factors must be taken into consideration:

1. Information Regarding the Process/Area of Concern

a. Secondary data (previous pilot studies)

b. Conceptual model (size/breadth and media of concern, variability sources, chemical/physical properties and distribution of contaminant)

2. Data Quality Information
a. Purpose of data collection
d. Statistical parameter of interest
b. Spatial/Temporal Boundaries
e. Tolerance for Potential Decision Errors
c. Preliminary variance estimates
f. Overall Precision Requirements

3. Constraints
a. Sampling/Analysis
b. Time/Schedule
c. Budget 
The number of sampling points must be established to ensure a strong (i.e., 95\%) confidence level of data correlation to represent the population with respect to the various experimental parameters and conditions to be analyzed. As such, the software spectrometer setting for each ICP-AES sample during every analytical sequence was selected to perform analytical combinations in triplicate. Ideally, it would be to extract three (3) sub-samples in triplicate. Due to budget limitations, the former methodology was applied as the most resource-effective design for the research project.

\subsubsection{Validation Check Parameters}

Triplicate readings and their mean concentrations in calibration units are generated in $\mathrm{mg} / \mathrm{L}$ by the ICP-AES software. During every ICP-AES analytical session, several quality control methods were applied, and evaluated to assess the calibration quality (Perkin Elmer Inc., 2010):

1. The triplicate concentrations of the median calibration standard $(50 \mathrm{mg} / \mathrm{L})$ (selected as an additional sample to act as a reference point of the data sample set) was within $5 \%$ of the known value;

2. The relative standard deviation $(\%-\mathrm{RSD})$ was well within the <3\% limit recommended; as well as the;

3. The correlation coefficient (CC) of each HMI analyte primary wavelength reported an average very close to unity.

These three parameters verified that the data are relatively accurate, highly reproducible, and the experimental replicates were reliable based on the calibration relationship established.

It is important to note that for purposes of cohesion, the validation of the QC protocol has been consolidated from each manuscript for each chapter study of the Dissertation document. 


\subsubsection{Influent Concentration Detection Trends}

Appendix A.5 outlines the procedure employed to prepare the HMI influent stock for all (single-, dual-, triple-, multi-) component system combinations. Based on the chemical and physical properties of the selected analytical grade nitrate salts (Table A.2), the conversion of the total influent concentration of $0.01 \mathrm{~N}(10 \mathrm{meq} / \mathrm{L})$ to $\mathrm{mg} / \mathrm{L}$ for each HMI was determined (Table A.5). These theoretical initial HMI concentration values are supported by various research efforts of current literature (Inglezakis et al., 2002; Inglezakis et al., 2003; Inglezakis et al., 2004; Stylianou et al., 2007a).

It is important to note that the experimentally determined HMI concentrations presented in $\mathrm{mg} / \mathrm{L}$ varied slightly from those theoretically expected. For all five HMIs combined in the major component systems (single-, dual-, triple-, multi-), the average \%-RSD of the experimental initial concentrations was approximately $2.45 \%$, which demonstrates laboratory consistency. Overall, the experimental initial concentrations were approximately $17.3 \%$ greater than that of the theoretically expected values. This discrepancy may be attributed to various factors, including the significantly small analytical grade nitrate salt mass (of less than $1 \mathrm{~g}$ ) required, the trace elements present in the concentrated nitric acid used for acidification, as well as the ICP-AES equipment settings (i.e., argon gas tank pressure, peristaltic pump maintenance, tubing, etc.), standards calibration and sample preparations. Interestingly, the multi-component $[\mathrm{M}]$ system combination concentrations had the lowest \%-RSD, and were closest to the theoretical influent concentrations; only one 50\% (DIL1) dilution for sample preparations is required (Appendix A.9). This trend points to the influence of dilution protocol. During every experimental laboratory session, the $\mathrm{pH}$ meter, analytical scale and pipettes $(0.1-1 \mathrm{~mL} ; 2-10 \mathrm{~mL})$ were calibrated on a standard, routine basis to minimize any associated impacts to the experimental data. Overall, consistency was demonstrated by all calculations conducted and results presented in the Dissertation document. 


\title{
4. EFFECTS OF OPERATION PARAMETERS \\ Effects of Operation Parameters on Heavy Metallic Ion \\ Removal from Mine Waste by Natural Zeolite \\ International Journal of Environmental Pollution and Remediation \\ 2018. 6(1). 10-24. doi:10.11159/ijepr.2018.002.
}

Fundamental components of this chapter were also presented as a paper at the WEAO2017 Technical Symposium ('Industrial Treatment A' Session); referenced in Appendix D of the Dissertation document.

\begin{abstract}
This study investigates the effects of particle size $(0.420-1.1410 \mathrm{~mm})$, dosage $(40,80 \mathrm{~g} / \mathrm{L})$, influent concentration (total $10 \mathrm{meq} / \mathrm{L}, 400 \mathrm{mg} / \mathrm{L}$ ), contact time (5-180, 270, $360 \mathrm{~min})$, settemperature $\left(20-32^{\circ} \mathrm{C}\right)$, and heat pre-treatment $\left(200,400,600^{\circ} \mathrm{C}\right)$ of natural zeolite on the removal efficiency of heavy metallic ions (HMIs); lead $\left(\mathrm{Pb}^{2+}\right)$, copper $\left(\mathrm{Cu}^{2+}\right)$, iron $\left(\mathrm{Fe}^{3+}\right)$, nickel $\left(\mathrm{Ni}^{2+}\right)$, and zinc $\left(\mathrm{Zn}^{2+}\right)$. The sorption process is performed in batch-mode with a $100 \mathrm{~mL}$ aqueous solution, acidified to a $\mathrm{pH}$ level of 2 with concentrated nitric $\left(\mathrm{HNO}_{3}\right)$ acid. For all experimental parameter conditions examined, the removal efficiency order follows: $\mathrm{Pb}^{2+}>>\mathrm{Fe}^{3+}>\mathrm{Cu}^{2+}>\mathrm{Zn}^{2+}>\mathrm{Ni}^{2+}$; the zeolite mineral exhibits the greatest preference towards the $\mathrm{Pb}^{2+}$ ion in all parameter trends. Overall, the removal efficiency is increased with decreasing particle size, as well as increasing dosage, contact time, and set-temperature. The operation is influenced by the studied parameters in the order of: influent concentration $>$ heat pre-treatment level $>$ dosage $>$ particle size $>$ contact time $>$ set-temperature.
\end{abstract}




\subsection{Introduction}

The industry holds great interest in the physico-chemical influential factors that dictate sorption efficiency of zeolite; which include particle size, initial concentration, $\mathrm{pH}$ level, and contact time. A smaller particle size of the sorbent material provides greater contact surface area, which improves the performance of the sorption process (Inglezakis et al., 2003; Acheampong and Meulepas, 2010); which may be attributed to diffusion as the rate-determining step (RDS) of the overall ion-exchange mechanism in the sorption process (Inglezakis et al., 2003). The effect of the dosage (solid-mass-to-solution-volume) on the uptake of heavy metal ions is wellestablished. An increase in dosage translates to an increase in the rate of uptake; although the amount sorbed per unit mass decreases, there is a higher availability of active sorption sites which sorb more HMIs from the solution (Motsi et al., 2009).

The initial concentration influences the removal efficiency due to the availability of functional groups on the specific surface to bind with the HMIs. This is primarily the case at higher concentrations, demonstrating a higher overall uptake given that the concentration difference is the driving force to overcome mass transfer resistance to metal ion transport between the solution and the sorbent surface (Acheampong and Meulepas, 2010). The pH influences the dissociation of the sorbent and solution chemistry, and affects surface charge of the sorbents and degree of ionization of different pollutants (Acheampong and Meulepas, 2010). This influence of acidity is particularly the case for HMIs that are in a rather low preference by zeolite; the initial $\mathrm{pH}$ must be attentively selected to ensure a balance among all ionic species. The goal is to avoid precipitation; for once precipitated, the ions of interest cannot be sorbed (Inglezakis et al., 2003).

The state of equilibrium is altered throughout the sorption process. Room temperature is preferred for analysis, although higher temperatures are assumed to enhance sorption with increased surface activities and solute kinetic energy (Motsi et al., 2009; Acheampong and Meulepas, 2010). Thermal treatment may enhance the sorption capacity, by removing the 'zeolitic water' present in the framework (Motsi et al., 2009); however, the dehydration of zeolite is an endothermic process, thereby causing 'activation' of the material (Mumpton et al., 1977) to a certain threshold, after which may lead to the structural collapse of the mineral (Motsi et al., 2009). 
The contact time is an important factor in the relationship of pollutants and sorbents. The rapid uptake of pollutants and equilibrium is established in a specific and limited period, which demonstrates efficiency of the sorbent for treatment. The mechanism study conducted by Sprynskyy et al. (2006) states that the sorption of HMIs by natural zeolite is a heterogeneous process with three distinct stages: (1) rapid uptake within the first 30 minutes of contact, (2) inversion due to desorption prevalence, and (3) slower increase in uptake. In the kinetic studies conducted by Motsi et al. (2009), the initial stage of rapid adsorption occurs within the first 40 contact minutes; when all of the adsorption sites are available for cations to interact, and when the concentration difference between the influent stock and sorbent-solution interface is very high. Inglezakis et al. (2002) tributes this period to ion-exchange in the micropores on the zeolite particles' surface. The predominance of desorption is most likely caused by slower diffusion of exchangeable cations within the internal zeolite crystalline structure, and consequently these preferred ions occupy the available exchange positions on the zeolite surface. During the third stage, the gradual deceleration of sorption in the micropores is caused by poor access as well as by more intensive sorption in comparison to the particles' surface. All of these factors are significant towards establishing the performance of any sorbent material (Acheampong and Meulepas, 2010).

In feasible treatments of industrial waste, it is essential to classify the degree of influence of each operational parameter on the overall system performance (Inglezakis et al., 2004). Therefore, the objective of this phase of the research project is to assess the sorption capacity of natural zeolite for the removal of the five HMIs selected, combined in various component systems. The operative conditions of particle size, zeolite dosage, influent concentration, contact time, set-temperature and heat pre-treatment level are investigated. This is of great importance, in order to harness the full potential of zeolite in tertiary treatment processes. 


\subsection{Materials and Methods}

Refer to Chapter 3 for the consolidated experimental methodology; sorbent material, sorbate solution, and analytical procedure. All analyses are conducted in batch-mode, creating the synthetic simple solute HMI solution in the various component system combinations at a 100-mL sorbate volume.

Table 4.1 summarizes the parameters investigated to determine their influence on the overall removal of the selected HMIs in this study. The heat pre-treatment was conducted by placing the cleaned zeolite into a pre-heated muffle furnace (NEY M-525 SII; Serial No. AKN 9403-108; $120 \mathrm{~V} ; 50 / 60 \mathrm{~Hz} ; 12.5 \mathrm{~A} ; 1500 \mathrm{~W}$; Barkmeyer Division, USA) at the three selected temperatures of $200^{\circ} \mathrm{C}, 400^{\circ} \mathrm{C}$, and $600^{\circ} \mathrm{C}$ (Motsi et al., 2009), for 1-hour; as outlined in the hydrothermal pre-treatment procedure in Appendix A.7.

Table 4.1 Operation Parameters and Conditions

\begin{tabular}{|c|c|}
\hline Parameter & Conditions \\
\hline \multirow{4}{*}{ Particle Size } & Single-Component Systems: $[\mathrm{P}],[\mathrm{C}],[\mathrm{F}],[\mathrm{N}],[\mathrm{Z}]$ \\
\hline & A $1.140-1.190 m m$ (pass No. 14 , retain No. 16 ) \\
\hline & В $0.707-0.841 \mathrm{~mm}$ (pass No. 20, retain No. 25$)$ \\
\hline & C $0.420-0.595 \mathrm{~mm}$ (pass No. 30 , retain No. 40$)$ \\
\hline \multirow{3}{*}{ Dosage } & Single-Component Systems: $[\mathrm{P}],[\mathrm{C}],[\mathrm{F}],[\mathrm{N}],[\mathrm{Z}]$ \\
\hline & Particle Size: D $(0.841-1.19 \mathrm{~mm})$ \\
\hline & Dosage: $4 \mathrm{~g} / 100 \mathrm{~mL}, 8 \mathrm{~g} / 100 \mathrm{~mL}$ \\
\hline \multirow{3}{*}{$\begin{array}{c}\text { Influent } \\
\text { Concentration }\end{array}$} & Systems: [P], [T], [M] \\
\hline & Particle Size: D \\
\hline & Concentrations: total $10 \mathrm{meq} / \mathrm{L}, 400 \mathrm{mg} / \mathrm{L}$ \\
\hline \multirow{3}{*}{ Contact Time } & Systems: [P], [D-PC], [D-PF], [T], [M] \\
\hline & Particle Size: D \\
\hline & Contact Time: $180,270,360$ minutes \\
\hline \multirow{4}{*}{ Set-Temperature } & Systems: $[\mathrm{T}],[\mathrm{M}]$ \\
\hline & Particle Size: D \\
\hline & Contact Time: 180 minutes \\
\hline & Set Temperature: $20^{\circ} \mathrm{C}, 24^{\circ} \mathrm{C}, 28^{\circ} \mathrm{C}, 32^{\circ} \mathrm{C}$ \\
\hline \multirow{3}{*}{$\begin{array}{c}\text { Heat } \\
\text { Pre-Treatment }\end{array}$} & Systems: $[\mathrm{P}],[\mathrm{T}],[\mathrm{M}]$ \\
\hline & Particle Size: D \\
\hline & Heat Pre-Treatment: $200^{\circ} \mathrm{C}, 400^{\circ} \mathrm{C}, 600^{\circ} \mathrm{C}$ \\
\hline
\end{tabular}




\subsection{Quality Control (QC) Protocol}

For all analytical sessions in this chapter, the triplicate concentration of the median standard $(50 \mathrm{mg} / \mathrm{L})$ detected an average of $51.37 \mathrm{mg} / \mathrm{L}$, and is within $5 \%$ of the known value; the percent relative standard deviation (\%-RSD) reported an average of $0.494 \%$, which is well within the $\leq 3 \%$ limit; the correlation coefficient of each HMI analyte primary wavelength generated an average of 0.9997 , which is very close to unity.

\subsection{Analysis}

\subsubsection{Particle Size and Dosage}

The particle size and dosage parameters are of paramount significance to this study, as well as to the industry that adopt sorption as a treatment method. Figure 4.1 displays the uptake of each HMI at 180 minutes of contact with zeolite. As expected, with a reduction in the particle size $\left(d_{p}\right)$ from $A$ to $C$, the uptake and percent removal increases. This trend is most prevalent for the HMI $\mathrm{Pb}^{2+}$, with a $45.6 \%$ decrease in concentration or a $15.2 \%$ increase in uptake from $d_{p, A}$ $(0.1872 \mathrm{meq} / \mathrm{g})$ to $\mathrm{d}_{\mathrm{p}, \mathrm{B}}(0.2157 \mathrm{meq} / \mathrm{g})$. However, this trend is not as prevalent from $\mathrm{d}_{\mathrm{p}, \mathrm{B}}$ to $\mathrm{d}_{\mathrm{p}, \mathrm{C}}$, with only a $3.98 \%$ in improved HMI uptake. This may be due to the greater particle size gradation range between $d_{p, A}$ and $d_{p, B}$ specifically, as well as a $40.5 \%$ decrease in nominal geometric mean diameter of $1.30 \mathrm{~mm}\left(\mathrm{~d}_{\mathrm{p}, \mathrm{A}}\right)$ to $0.77 \mathrm{~mm}\left(\mathrm{~d}_{\mathrm{p}, \mathrm{B}}\right)$. Based on the sieve distribution presented in Table 3.4 of Chapter 3, an average of $10 \%$ per mesh range was detected for particle sizes B and C. In order to eliminate skater/variability, and to maintain a controlled environment, the particle size selected to observe the other experimental parameters is selected between $A$ and $B$, denoted hereon in as size $D\left(d_{p, D}\right)$. Based on these initial observations in the removal trends by particle size, the $d_{p, D}$ was considered a more feasible and conservative range moving forward; with a nominal geometric mean diameter of $1.00 \mathrm{~mm}$ (Mullin, 2001). 


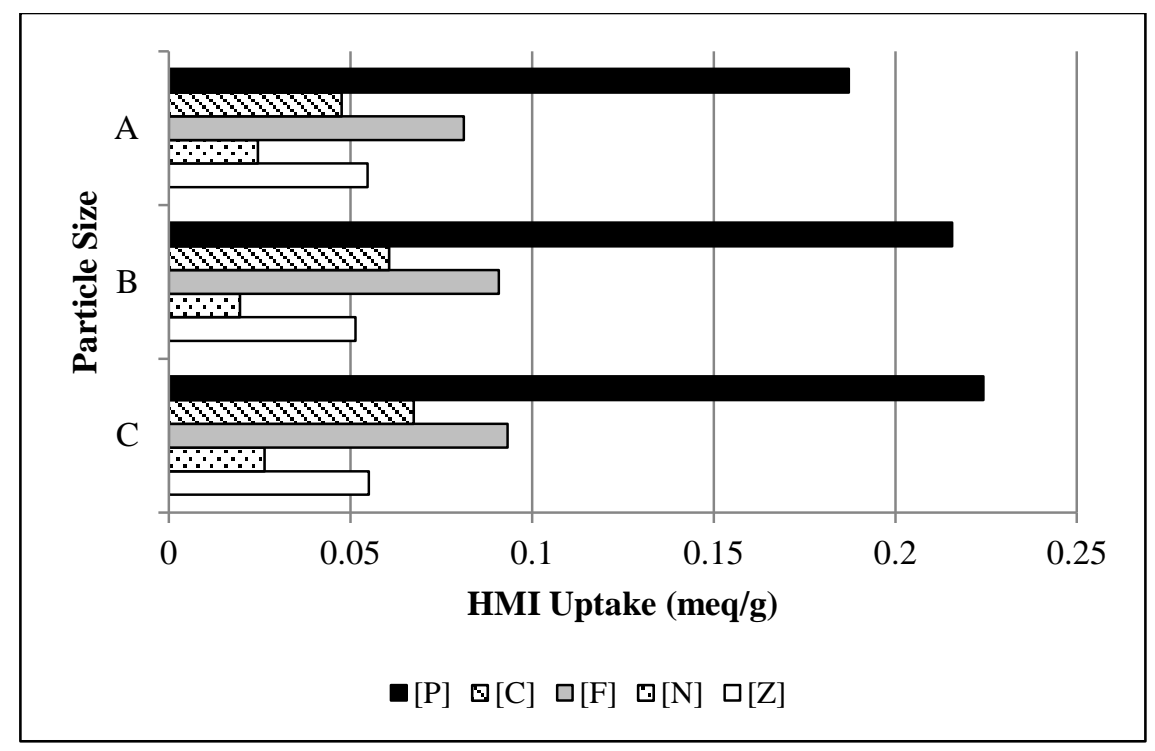

Figure 4.1 HMI Uptake based on Particle Size Parameter

Figure 4.2 displays the overall percent removal of each HMI (in single-component solutions) at 180 minutes of contact with natural zeolite by increasing the sorbent dosage from 4 $\mathrm{g}$ to $8 \mathrm{~g}$, for every $100 \mathrm{~mL}$ of sorbate volume. As illustrated, when the dosage increases (doubled), the percent removal increases substantially; which is attributed to higher site uptake availability (Oter and Akcay, 2007). The HMI effluent concentration at a contact time of 180 minutes is reduced for $\mathrm{Cu}^{2+}$ at $19.9 \%, \mathrm{Fe}^{3+}$ at $35.9 \%$, and significantly for $\mathrm{Pb}^{2+}$ at $82.4 \%$. Additionally, the overall removal efficiency of the selected $d_{p, D}$ falls within the range achieved of $\mathrm{d}_{\mathrm{p}, \mathrm{A}}$ and $\mathrm{d}_{\mathrm{p}, \mathrm{B}}$; demonstrating experimental continuity.

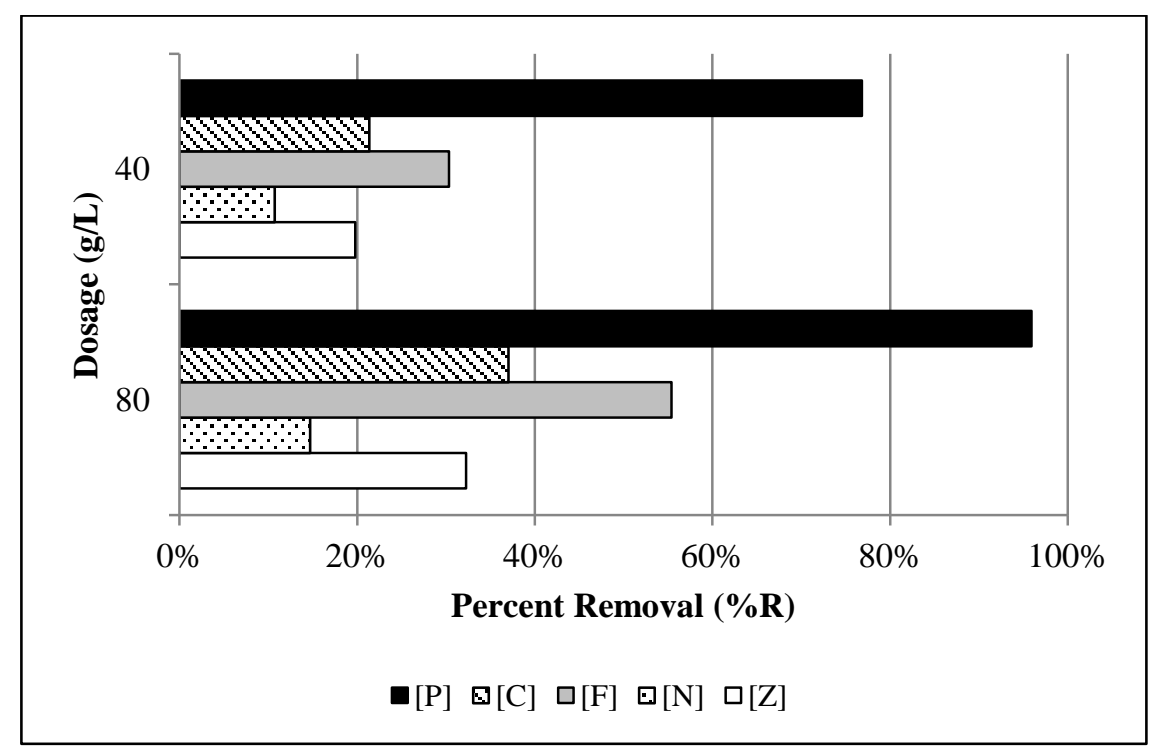

Figure 4.2 HMI Percent Removal based on Dosage Parameter 
Kinetic modelling is a powerful tool to assess the performance of sorbent materials and to better comprehend the fundamental mechanisms involved in the sorption process. The sorption rate depends on the amount of ions on the sorbent surface at time $t$ and what is sorbed when an equilibrium state is reached. The models are classified as either reaction-type or diffusion-type (film, intra-particle) (Oter and Akcay, 2007); both models have been thoroughly investigated and have demonstrated strong correlation (Bektas and Kara, 2004; Ho and Ofomaja, 2006; Oter and Akcay, 2007; Qiu et al., 2009).

The reaction-type known as the pseudo-second-order (PSO) kinetic model has welldemonstrated this rate process of various contaminants, including metal ions and organic substances in an aqueous state (Qiu et al., 2009; Jovanovic et al., 2012). This model implies that the RDS is by chemical adsorption (chemisorption). It is represented in Equation 4.1 and by applying the boundary conditions of $t=0 \rightarrow q_{t}=0$ and $t=t \rightarrow q_{t}=q_{t}$, its linearized form is presented in Equation 4.2 (Sprynskyy et al., 2006; Ho and Ofomaja, 2006; Qiu et al., 2009; Motsi et al., 2011):

$$
\begin{aligned}
\frac{d q_{t}}{d t} & =k_{2}\left(q_{e}-q_{t}\right)^{2} \\
\frac{t}{q_{t}} & =\frac{t}{q_{e}}+\frac{1}{k_{2} q_{e}^{2}}
\end{aligned}
$$

where $\mathrm{h}=\mathrm{k}_{2} \mathrm{q}_{\mathrm{e}}^{2}$ is the initial sorption rate (in meq/g-min) as $\mathrm{t}$ approaches zero (Ho and Ofomaja, 2006), and $\mathrm{k}_{2}$ is the PSO rate constant (in $\mathrm{g} / \mathrm{meq} \cdot \mathrm{min}$ ). These constants are determined by a plot of the linearized form of $t / q_{t}$ versus $t$ (Ho and Ofomaja, 2006; Qiu et al., 2009). The PSO rate constants and correlation coefficients are summarized in Table 4.2 and Table 4.3 for the particle size and dosage parameters, respectively. Based on the linearized form of Equation 4.2, the slope (m) and y-intercept (b) values are interpreted to determine the theoretical sorption at equilibrium $\left(\mathrm{q}_{\mathrm{e}}\right.$ in meq/g). The experimental sorption at 180 minutes $\left(\mathrm{q}_{180}\right.$ in meq/g) of contact is also presented. 
Table 4.2 PSO - Particle Size Data

\begin{tabular}{cccccc}
\hline \multicolumn{7}{c}{ Size A } \\
\hline System & $\mathbf{q}_{\mathbf{1 8 0}}$ & $\mathbf{C C}$ & $\mathbf{m}$ & $\mathbf{b}$ & $\mathbf{q}_{\mathbf{e}}$ \\
\hline$[\mathrm{P}]$ & 0.1872 & 0.9840 & 4.374 & 216.46 & 0.2286 \\
\hline$[\mathrm{C}]$ & 0.0476 & 0.8193 & 12.780 & 1445.40 & 0.0782 \\
\hline$[\mathrm{F}]$ & 0.0813 & 0.9741 & 11.002 & 372.35 & 0.0909 \\
\hline$[\mathrm{N}]$ & 0.0245 & 0.9141 & 31.057 & 969.75 & 0.0322 \\
\hline$[\mathrm{Z}]$ & 0.0548 & 0.7413 & 9.569 & 1420.10 & 0.1045 \\
\hline \multicolumn{5}{c}{ Size B } \\
\hline System & $\mathbf{q}_{\mathbf{1 8 0}}$ & $\mathbf{C C}$ & $\mathbf{m}$ & $\mathbf{b}$ & $\mathbf{q}_{\mathbf{e}}$ \\
\hline$[\mathrm{P}]$ & 0.2157 & 0.9970 & 3.856 & 147.15 & 0.2594 \\
\hline$[\mathrm{C}]$ & 0.0607 & 0.9866 & 13.611 & 626.99 & 0.0735 \\
\hline$[\mathrm{F}]$ & 0.0908 & 0.9934 & 10.190 & 202.53 & 0.0981 \\
\hline$[\mathrm{N}]$ & 0.0196 & 0.9872 & 47.121 & 298.41 & 0.0212 \\
\hline$[\mathrm{Z}]$ & 0.0514 & 0.9623 & 16.317 & 795.49 & 0.0613 \\
\hline \multicolumn{7}{c}{ Size C } \\
\hline System & $\mathbf{q}_{\mathbf{1 8 0}}$ & $\mathbf{C C}$ & $\mathbf{m}$ & $\mathbf{b}$ & $\mathbf{q} \mathbf{q}$ \\
\hline$[\mathrm{P}]$ & 0.2242 & 0.9964 & 3.783 & 110.79 & 0.2644 \\
\hline$[\mathrm{C}]$ & 0.0674 & 0.9700 & 13.942 & 373.99 & 0.0717 \\
\hline$[\mathrm{F}]$ & 0.0933 & 0.9976 & 10.067 & 155.73 & 0.0993 \\
\hline$[\mathrm{N}]$ & 0.0263 & 0.9942 & 37.924 & 274.89 & 0.0264 \\
\hline$[\mathrm{Z}]$ & 0.0550 & 0.9646 & 17.211 & 430.21 & 0.0581 \\
\hline
\end{tabular}

Table 4.3 PSO - Dosage Data

\begin{tabular}{cccccc}
\hline \multicolumn{5}{c}{ Dosage 40 } \\
\hline System & $\mathbf{q}_{\mathbf{1 8 0}}$ & $\mathbf{C C}$ & $\mathbf{m}$ & $\mathbf{b}$ & $\mathbf{q} \mathbf{e}$ \\
\hline$[\mathrm{P}]$ & 0.1919 & 0.9926 & 4.098 & 217.01 & 0.2440 \\
\hline$[\mathrm{C}]$ & 0.0533 & 0.9291 & 15.750 & 836.09 & 0.0635 \\
\hline$[\mathrm{F}]$ & 0.0757 & 0.9708 & 11.872 & 419.08 & 0.0842 \\
\hline$[\mathrm{N}]$ & 0.0268 & 0.9806 & 34.919 & 739.14 & 0.0286 \\
\hline$[\mathrm{Z}]$ & 0.0494 & 0.9147 & 15.237 & 1106.10 & 0.0656 \\
\hline \multicolumn{5}{c}{ Dosage 80 } \\
\hline System & $\mathbf{q}_{\mathbf{1 8 0}}$ & $\mathbf{C C}$ & $\mathbf{m}$ & $\mathbf{b}$ & $\mathbf{q}_{\mathbf{e}}$ \\
\hline$[\mathrm{P}]$ & 0.1198 & 0.9986 & 7.343 & 174.00 & 0.1362 \\
\hline$[\mathrm{C}]$ & 0.0463 & 0.9821 & 17.211 & 926.12 & 0.0581 \\
\hline$[\mathrm{F}]$ & 0.0691 & 0.9899 & 12.893 & 344.90 & 0.0776 \\
\hline$[\mathrm{N}]$ & 0.0184 & 0.9967 & 50.300 & 655.14 & 0.0199 \\
\hline$[\mathrm{Z}]$ & 0.0403 & 0.6369 & 13.507 & 1771.00 & 0.0740 \\
\hline & & & & &
\end{tabular}

As demonstrated by the coefficients (CC), a strong correlation is established for all HMIs for both parameters. For all HMIs on average, the particle size $\mathrm{q}_{180}$ reaches the theoretical $\mathrm{q}_{\mathrm{e}}$ uptake of $92.3 \%$ for $d_{p, A}, 86.9 \%$ for $d_{p, B}$, and $93.5 \%$ for $d_{p, C}$; the dosage $\mathrm{q}_{180}$ reaches the theoretical $\mathrm{q}_{\mathrm{e}}$ uptake on average of $84.3 \%$ for dosage $40 \mathrm{~g} / \mathrm{L}$ and $80.7 \%$ for dosage $80 \mathrm{~g} / \mathrm{L}$. The particle size 
uptake rate trends in Table $\mathbf{4 . 2}$ are systematically consistent; with the $\mathrm{q}_{\mathrm{e},[\mathrm{P}]}$ within $\mathbf{5 \%}$ of the theoretical maximum $0.25 \mathrm{meq} / \mathrm{g}$ threshold for total HMIs.

The dosage level is not directly proportional to the sorption removal efficiency. The removal efficiency of $\mathrm{Pb}^{2+}$ improves from 76.8 to $95.9 \%$; however, the $\mathrm{q}_{180}$ uptake has decreased from 0.1919 to $0.1198 \mathrm{meq} / \mathrm{g}$, and the theoretically anticipated $\mathrm{q}_{\mathrm{e}}$ uptake at equilibrium decreases from 0.2440 to $0.1362 \mathrm{meq} / \mathrm{g}$, comparing a dosage of 40 to $80 \mathrm{~g} / \mathrm{L}$, respectively. This may be attributed to the very rapid uptake of the first stage of sorption. The two HMIs preferred by zeolite in this study exhibit a faster initial sorption rate $(\mathrm{h})$; for $\mathrm{Pb}^{2+}$, the rate increases from 0.0046 to $0.0057 \mathrm{meq} / \mathrm{g} \cdot \mathrm{min}$ and for $\mathrm{Fe}^{3+}$, this rate increases from 0.0024 to $0.0029 \mathrm{meq} / \mathrm{g} \cdot \mathrm{min}$; comparing dosage 40 to $80 \mathrm{~g} / \mathrm{L}$, respectively. This finding in conjunction with the lower overall expected uptake at equilibrium demonstrates that the dosage $80(8 \mathrm{~g} / 100 \mathrm{~mL})$ has reached its threshold of available active sorption sites. A higher removal at a faster rate comes at a cost of consuming more zeolite material; with the dosage 40 (4 g/100 mL) considered more economically feasible (Beyazit, 2013).

In accordance with the fundamental principles of sorption (adsorption and ion-exchange), when intra-particle diffusion (IPD) as considered as the rate-determining step, the sorption rate is proportional to $\bar{D} / d_{p}^{2}$; where $\bar{D}$ is the diffusion coefficient of a specific HMI. Since the $d_{p}$ should not affect either the equilibrium state or the $\overline{\mathrm{D}}$, higher sorption rates should be observed for smaller particle sizes. However, smaller particle sizes may exhibit lower rates, due to lower effective $\bar{D}$ values, caused by structural problems or pore clogging (Inglezakis et al., 2004). It is important to note that the natural (as-received) zeolite mineral sample is put through a systematic cleaning cycle, thoroughly washing before use. Therefore, pore clogging is not expected to affect the diffusion coefficients which are considered to be constant regardless of particle size. Then, with intra-particle diffusion considered as the controlling step, the exchange rate should be increased by decreasing particle size (Inglezakis et al., 2004); as demonstrated. Based on the trends observed under the testing conditions, the ideal levels of these two parameters are selected moving forward in this study. 


\subsubsection{Influent Concentration}

In addition to maintaining a total $10 \mathrm{meq} / \mathrm{L}$ initial concentration, this component of the study is also conducted at $400 \mathrm{mg} / \mathrm{L}$ for each $\mathrm{HMI}$, based on the median range of conversion from $\mathrm{meq} / \mathrm{L}$ to $\mathrm{mg} / \mathrm{L}$ concentrations for a majority of the HMI investigated throughout this research endeavour; or single-lead [P], triple- $[\mathrm{T}]$, and multi- $[\mathrm{M}]$ component system combinations (Table 4.4).

Table 4.4 The HMI Removal Variation by Influent Concentration

\begin{tabular}{|c|c|c|c|c|c|c|}
\hline \multirow{3}{*}{ System } & \multirow{3}{*}{ HMI } & \multicolumn{3}{|c|}{$\begin{array}{c}\text { Total } \\
10 \mathrm{meq} / \mathrm{L}\end{array}$} & \multicolumn{2}{|c|}{$\begin{array}{l}400 \mathrm{mg} / \mathrm{L} \\
\text { per } \mathrm{HMI}\end{array}$} \\
\hline & & \multicolumn{2}{|c|}{$\mathrm{q}_{180}$} & \multirow{2}{*}{$\% \mathrm{R}$} & \multirow{2}{*}{$\begin{array}{l}\mathrm{q}_{180} \\
\mathrm{mg} / \mathrm{g}\end{array}$} & \multirow{2}{*}{$\% \mathrm{R}$} \\
\hline & & $\mathrm{mg} / \mathrm{g}$ & $\mathrm{meq} / \mathrm{g}$ & & & \\
\hline$[\mathrm{P}]$ & $\mathrm{Pb}^{2+}$ & 23.31 & 0.192 & 76.8 & 9.36 & 95.1 \\
\hline \multirow{4}{*}[\mathrm{T}]{} & $\mathrm{Pb}^{2+}$ & 9.01 & 0.075 & 90.2 & 7.35 & 79.9 \\
\hline & $\mathrm{Cu}^{2+}$ & 0.64 & 0.016 & 19.0 & 1.04 & 11.4 \\
\hline & $\mathrm{Fe}^{3+}$ & 0.85 & 0.041 & 49.5 & 1.73 & 18.3 \\
\hline & TOTAL & - & 0.132 & - & - & - \\
\hline \multirow{6}{*}[\mathrm{M}]{} & $\mathrm{Pb}^{2+}$ & 5.52 & 0.047 & 94.0 & 7.62 & 79.7 \\
\hline & $\mathrm{Cu}^{2+}$ & 0.41 & 0.011 & 21.9 & 0.75 & 8.42 \\
\hline & $\mathrm{Fe}^{3+}$ & 0.58 & 0.028 & 56.2 & 1.51 & 15.6 \\
\hline & $\mathrm{Ni}^{2+}$ & 0.15 & 0.005 & 9.10 & 0.18 & 1.84 \\
\hline & $\mathrm{Zn}^{2+}$ & 0.30 & 0.008 & 16.5 & 0.41 & 4.58 \\
\hline & TOTAL & - & 0.099 & - & - & - \\
\hline
\end{tabular}

The difference in the removal of each HMI investigated when the influent concentration is set to meq/L versus $\mathrm{mg} / \mathrm{L}$ is evident. The trends observed are consistent with the literature; the amount in $\mathrm{mg}$ of $\mathrm{Pb}^{2+}$ ions available for uptake by zeolite decreases, theoretically from 1036 $\mathrm{mg} / \mathrm{L}$ to $400 \mathrm{mg} / \mathrm{L}$ and the amount of the other four ions (in $\mathrm{mg}$ ) has increased with this conversion of influent concentration. Oter and Akcay (2007) demonstrated consistent findings, as the initial concentration increases, the amount of sorbed HMI increases, while the percent of sorbed HMI decreases for all ions.

Inglezakis et al. (2003) demonstrated that dilution leads to an increase in the volume of treated solution to breakthrough (5-10\% of the influent concentration) in continuous column configuration; the magnitude of which depends on the specific metal exchanged. This finding can be attributed to the increase of selectivity in the ion-exchange mechanism of sorption by dilution. The valences of the exchanging cations have a strong effect on ion-exchange at equilibrium, and consequently on the removal efficiency. This attribute is referred to as the "concentration-valency effect". It is theoretically recognized that when the exchanging ions are 
not of equal valence, the equilibrium is a function of the total concentration; at higher concentrations, this process prefers the uptake of the lower charged cations and subsequently excludes higher charged cations from the sorbent (Inglezakis et al., 2003). The cations present in the sorbent have valences that differ from those in solution. Consequently, as the dilution increases, the selectivity of the sorbent for the ion with a higher valence also increases. Accordingly, comparative analysis of various metal ions should be conducted at the same normality and temperature, in order to minimize the changes observed in isotherm configuration with dilution (Inglezakis et al., 2003).

\subsubsection{Contact Time and Set-Temperature}

With $[\mathrm{P}],[\mathrm{T}]$, and $[\mathrm{M}]$ component system combinations at total $10 \mathrm{meq} / \mathrm{L}$ influent concentration: (1) the contact time was extrapolated from 3 hours to 4.5 and 6 hours (Table 4.5), and (2) the set-temperature was evaluated to an adjusted range of 20 to $32^{\circ} \mathrm{C}$ at 180 contact minutes (Table 4.6).

Table 4.5 The HMI Removal Variation by Contact Time at $22^{\circ} \mathrm{C}$ Set-Temperature

\begin{tabular}{|c|c|c|c|c|c|c|c|}
\hline \multirow{3}{*}{ System } & \multirow{3}{*}{ HMI } & \multicolumn{6}{|c|}{ Contact Time (mins) } \\
\hline & & \multicolumn{2}{|c|}{180} & \multicolumn{2}{|c|}{270} & \multicolumn{2}{|c|}{360} \\
\hline & & $\mathrm{q}_{180}$ & $\% \mathrm{R}$ & $\mathrm{q}_{270}$ & $\% \mathrm{R}$ & $\mathrm{q}_{360}$ & $\% \mathrm{R}$ \\
\hline$[\mathrm{P}]$ & $\mathrm{Pb}^{2+}$ & 0.2106 & 84.4 & 0.2235 & 89.5 & 0.2299 & 92.1 \\
\hline \multirow{3}{*}[\mathrm{PC}]{} & $\mathrm{Pb}^{2+}$ & 0.116 & 92.9 & 0.119 & 95.3 & 0.120 & 96.5 \\
\hline & $\mathrm{Cu}^{2+}$ & 0.025 & 19.7 & 0.031 & 24.9 & 0.034 & 27.3 \\
\hline & TOTAL & 0.1405 & - & 0.1500 & - & 0.1546 & - \\
\hline \multirow{3}{*}[\mathrm{PF}]{} & $\mathrm{Pb}^{2+}$ & 0.109 & 87.1 & 0.114 & 91.7 & 0.118 & 94.3 \\
\hline & $\mathrm{Fe}^{3+}$ & 0.054 & 43.3 & 0.060 & 47.7 & 0.064 & 51.5 \\
\hline & TOTAL & 0.1629 & - & 0.1740 & - & 0.1820 & - \\
\hline \multirow{4}{*}[\mathrm{T}]{} & $\mathrm{Pb}^{2+}$ & 0.076 & 91.7 & 0.079 & 94.7 & 0.080 & 96.0 \\
\hline & $\mathrm{Cu}^{2+}$ & 0.018 & 21.4 & 0.022 & 26.8 & 0.025 & 29.8 \\
\hline & $\mathrm{Fe}^{3+}$ & 0.041 & 48.9 & 0.046 & 55.4 & 0.049 & 58.6 \\
\hline & TOTAL & 0.1347 & - & 0.1471 & - & 0.1534 & - \\
\hline \multirow{6}{*}[\mathrm{M}]{} & $\mathrm{Pb}^{2+}$ & 0.047 & 95.1 & 0.048 & 96.6 & 0.049 & 97.3 \\
\hline & $\mathrm{Cu}^{2+}$ & 0.013 & 26.4 & 0.015 & 30.0 & 0.016 & 32.9 \\
\hline & $\mathrm{Fe}^{3+}$ & 0.029 & 58.8 & 0.031 & 62.5 & 0.033 & 67.0 \\
\hline & $\mathrm{Ni}^{2+}$ & 0.005 & 9.60 & 0.005 & 9.81 & 0.005 & 10.0 \\
\hline & $\mathrm{Zn}^{2+}$ & 0.011 & 21.6 & 0.013 & 25.2 & 0.014 & 27.8 \\
\hline & TOTAL & 0.1055 & - & 0.1118 & - & 0.1172 & - \\
\hline
\end{tabular}


Table 4.6 The HMI Removal Variation by Set-Temperature at 180 Contact Minutes

\begin{tabular}{|c|c|c|c|c|c|c|c|c|c|}
\hline & \multirow{3}{*}{ HMI } & \multicolumn{8}{|c|}{ Set-Temperature $\left({ }^{\circ} \mathrm{C}\right)$} \\
\hline & & \multicolumn{2}{|c|}{20} & \multicolumn{2}{|c|}{24} & \multicolumn{2}{|c|}{28} & \multicolumn{2}{|c|}{32} \\
\hline & & $\mathrm{q}_{180}$ & $\% \mathrm{R}$ & $\mathrm{q}_{180}$ & $\% \mathrm{R}$ & $\mathrm{q}_{180}$ & $\% \mathrm{R}$ & $\mathrm{q}_{180}$ & $\% \mathrm{R}$ \\
\hline \multirow{4}{*}[\mathrm{T}]{} & $\mathrm{Pb}^{2+}$ & 0.075 & 90.5 & 0.076 & 91.8 & 0.076 & 91.8 & 0.077 & 92.8 \\
\hline & $\mathrm{Cu}^{2+}$ & 0.018 & 21.8 & 0.019 & 23.2 & 0.020 & 24.4 & 0.022 & 26.7 \\
\hline & $\mathrm{Fe}^{3+}$ & 0.041 & 49.6 & 0.041 & 49.0 & 0.043 & 51.8 & 0.045 & 53.7 \\
\hline & TOTAL & 0.1347 & - & 0.1366 & - & 0.1398 & - & 0.1442 & - \\
\hline \multirow{6}{*}[\mathrm{M}]{} & $\mathrm{Pb}^{2+}$ & 0.047 & 94.8 & 0.048 & 95.3 & 0.048 & 95.3 & 0.048 & 95.7 \\
\hline & $\mathrm{Cu}^{2+}$ & 0.013 & 25.4 & 0.014 & 27.6 & 0.014 & 28.0 & 0.015 & 30.0 \\
\hline & $\mathrm{Fe}^{3+}$ & 0.030 & 60.0 & 0.030 & 60.0 & 0.031 & 61.6 & 0.031 & 62.8 \\
\hline & $\mathrm{Ni}^{2+}$ & 0.006 & 12.7 & 0.007 & 13.4 & 0.007 & 13.4 & 0.007 & 13.3 \\
\hline & $\mathrm{Zn}^{2+}$ & 0.011 & 22.0 & 0.012 & 24.9 & 0.013 & 25.1 & 0.014 & 27.3 \\
\hline & TOTAL & 0.1074 & - & 0.1106 & - & 0.1117 & - & 0.1144 & - \\
\hline
\end{tabular}

When the initial concentration data of Table 4.4 is compared to the contact time comparison in Table 4.5 at 180 contact minutes, the removal efficiency is similarly on trend. Only a 5.94\% average percent difference in the uptake of total HMIs of $[\mathrm{P}],[\mathrm{T}]$, and $[\mathrm{M}]$ is detected. When this same comparison is made with $20^{\circ} \mathrm{C}$ uptake of $[\mathrm{T}]$ and $[\mathrm{M}]$ data of Table 4.6 (a temperature below the controlled $22^{\circ} \mathrm{C}$ ), only a $4.85 \%$ average percent difference in the uptake of total HMIs is detected; these observations demonstrate continuity and repeatability of the experimental procedure. To visualize the influence of both operating parameters, Figure 4.3 and Figure 4.4 display the total HMI uptake (meq/g) with respect to extrapolated contact time and settemperature, respectively.

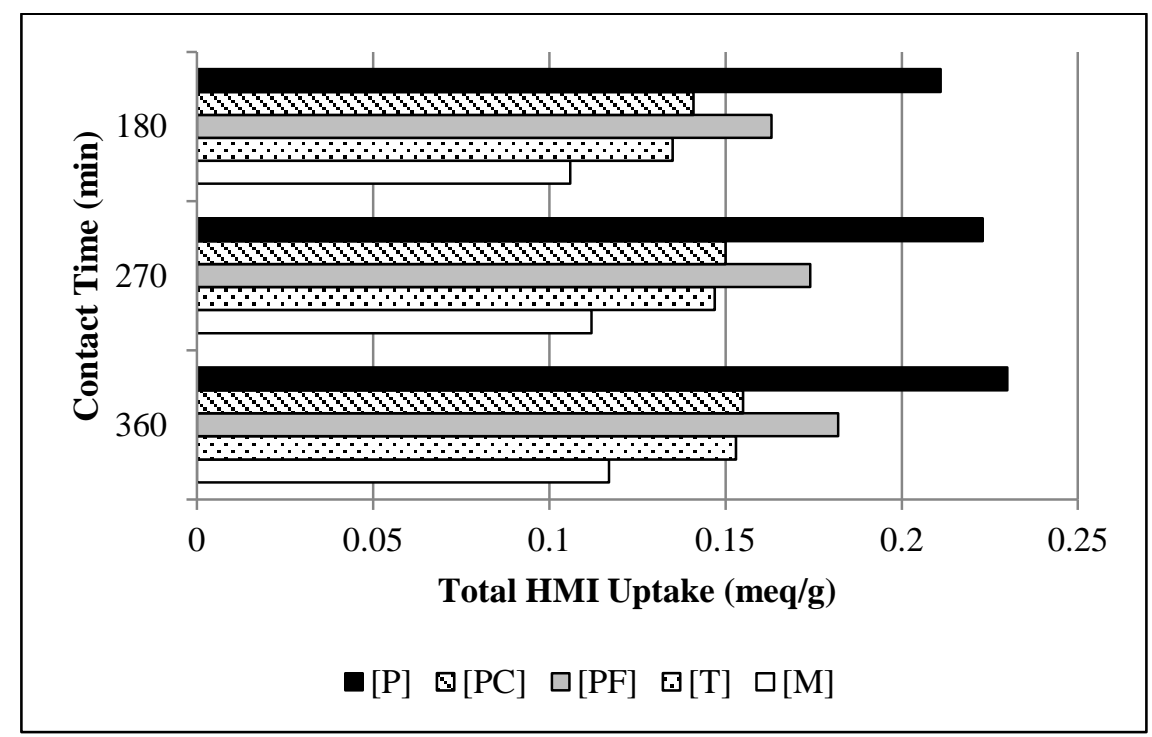

Figure 4.3 Total HMI Uptake based on Contact Time Parameter 


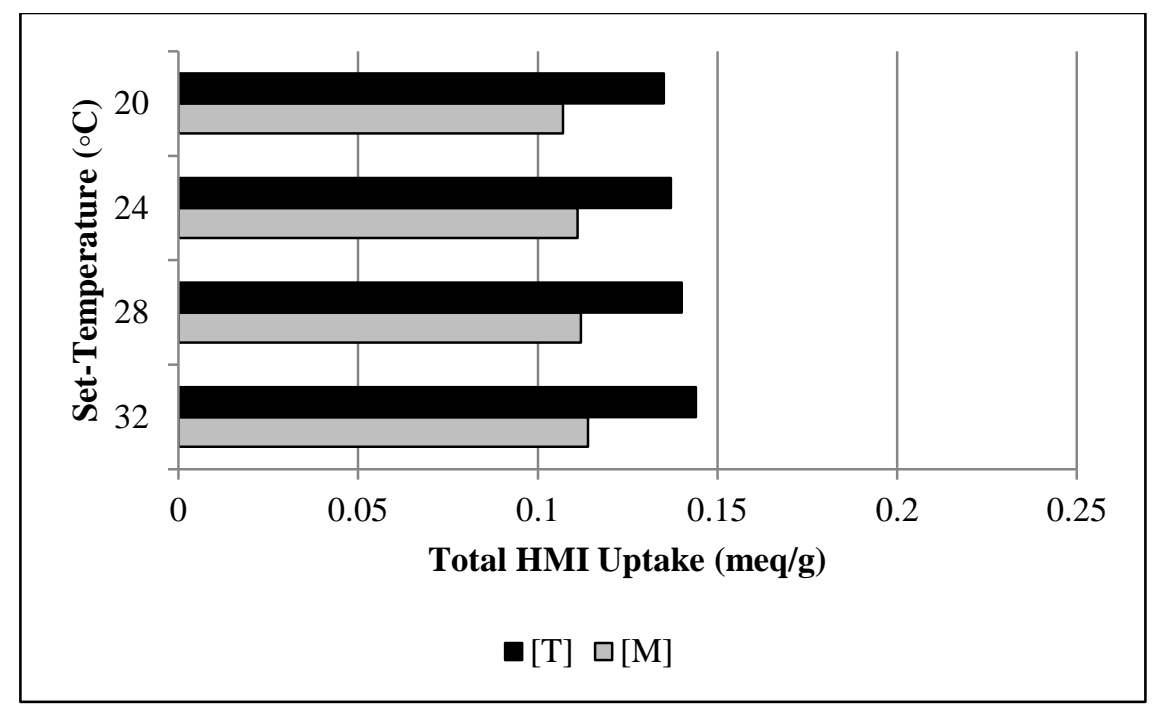

Figure 4.4 Total HMI Uptake based on Set-Temperature Parameter

A greater increase in uptake of the total HMIs occurs from 180 to 270 minutes by, on average $0.010 \mathrm{meq} / \mathrm{g}$ compared to $0.006 \mathrm{meq} / \mathrm{g}$ from 270 to 360 minutes. This demonstrates the expected proportionality between uptake and contact time. For $\mathrm{Pb}^{2+}$, the theoretical $\mathrm{q}_{\mathrm{e},[\mathrm{P}]}$ of $0.2440 \mathrm{meq} / \mathrm{g}$ by the PSO model (Table 4.3) is experimentally supported by the $\mathrm{q}_{360}$ of 0.230 meq/g. Research conducted by Oter and Akcay (2007) demonstrates that equilibrium is attained for $\mathrm{Cu}^{2+}$ and $\mathrm{Zn}^{2+}$ in approximately 6 contact hours, while more rapidly for $\mathrm{Pb}^{2+}$ and $\mathrm{Ni}^{2+}$ at only 1 contact hour. As such, the uptake threshold quickly approaches equilibrium at 360 minutes of contact.

The results in Table $\mathbf{4 . 6}$ are on trend, with a direct proportionality between the systematic increase in set-temperature and uptake. This supports the fact that the sorbent's structure and surface functional groups are influenced by temperature between $20-35^{\circ} \mathrm{C}$, observed by the overall sorption capacity (Sprynskyy et al., 2006). However, the impact of set-temperature is not as significant within this selected range of study conditions. 


\subsubsection{Hydrothermal Pre-Treatment}

The hydrothermal stability of zeolites establishes the operational lifetime of a material, as well as degradation and regeneration conditions (Kostandyan et al., 1982). It is a measure of the structural changes that occur when exposed to water vapour at high temperatures and pressures (Kostandyan et al., 1982). This characteristic depends primarily on the type of zeolite, the Si/Al ratio, as well as the divalent/monovalent ratio and nature of cations entering the framework (Kostandyan et al., 1982; Langella et al., 2003). The total dehydration of zeolite occurs at a temperature that significantly exceeds the boiling point of water. This process is therefore associated with considerable expenditures of energy used to break bonds holding water molecules in the intra-crystalline channels of zeolite as well as to overcome the diffusion energy barrier of water molecules in the crystal framework channels (Kostandyan et al., 1982). The structural changes that occur are influenced by the degree of participation of water molecules in the energy balance of zeolite. Therefore, water molecules positioned in cavities and channels of the framework contribute to the compensation of the non-uniformly distributed charge of the silicate framework and cations. When water is separated from the crystalline lattice, the charge distribution breaks down. This leads to a deformation of the framework and variation in the mobile cations' positions (Kostandyan et al., 1982).

Motsi et al. (2009) investigated the uptake efficiency of natural zeolite for the HMIs of $\mathrm{Fe}^{3+}$, $\mathrm{Cu}^{2+}, \mathrm{Mn}^{2+}$ and $\mathrm{Zn}^{2+}$. The effects of heat pre-treatment were examined with exposure to a muffle furnace at $200^{\circ} \mathrm{C}, 400^{\circ} \mathrm{C}$, and $800^{\circ} \mathrm{C}$ for 30 minutes. The pre-treated zeolite was then in contact with the HMIs in single-component solutions for 6 hours. It was observed that the specific surface area is improved when treated at $200^{\circ} \mathrm{C}$. An increase in both the adsorption rate and capacity due to this thermal treatment is caused by the removal of water from internal channels, which leave them vacant. However, this trend is minimized beyond this temperature threshold. The structure collapses and the porosity inevitably decreases. The rate of adsorption by calcined zeolite is faster compared to untreated zeolite, but the efficiency decreases for zeolite exposed to very high temperatures (Motsi et al., 2009). 
Water is removed continuously and reversibly, both partially and completely (Kostandyan et al., 1982; Langella et al., 2003), when exposed to heat from air at room temperature. When exposed to heat at approximately $350-400^{\circ} \mathrm{C}$ (Mumpton, 1977; Breck, 1974; Yörükoğulları et al., 2010), the water is eliminated, and the cations fall back into positions on the inner surface of channels and central cavities of the zeolite structure. Dehydration of zeolite is an endothermic process, thereby causing 'activation' of the material (Mumpton, 1977) between $250-400^{\circ} \mathrm{C}$ (Kostandyan et al., 1982), at approximately $350^{\circ} \mathrm{C}$ (Breck, 1974); with a structural stability of up to $750^{\circ} \mathrm{C}$ (Breck, 1974; Margeta et al., 2013). Research has also revealed that the relationship between the dehydration mechanism of zeolite and positions occupied by aluminum and cations in its structure have an effect on the thermal stability. Thermal treatment of zeolite between 500$600^{\circ} \mathrm{C}$ causes the loss of one $\mathrm{H}_{2} \mathrm{O}$ molecule from two nearby $\mathrm{OH}^{-}$or one $\mathrm{H}_{2} \mathrm{O}$ molecule for every two tetrahedral $\mathrm{Al}$ atoms in its structure. This temperature range instigates a loss of oxygen atoms in the framework, producing structural vacancies (Langella et al., 2003). Beyond this thermal threshold, the crystalline structure breaks down and the clinoptilolite becomes an amorphous solid (Breck, 1974; Kostandyan et al., 1982).

Langella et al. (2003) investigated the three thermal behavioural types of zeolites. This work emphasizes that reversible dehydration with minimal framework contraction would be observed upon heating up to approximately $230^{\circ} \mathrm{C}$ (Type-1) and $280^{\circ} \mathrm{C}$ (Type-2), while irreversible structural changes hinders rehydration at a range of $230-260^{\circ} \mathrm{C}$ (Type-1) and $280-$ $400^{\circ} \mathrm{C}$ (Type-2). Also, heat pre-treatment greater than $450^{\circ} \mathrm{C}$ (Type-1) and $550^{\circ} \mathrm{C}$ (Type-2) causes a thermally induced collapse of the zeolite structure. Behavioural Type-3 exhibits continuous reversible dehydration with only very small structural contraction; the framework is not destroyed at an exposure of up to $750^{\circ} \mathrm{C}$. High aluminum and alkaline-earth contents give rise to Type-1. An increase in silicon and/or alkaline-earth cations leads to a progressive change in thermal behavior in the order of Type- 1 to -2 to -3 . The study presented in the Dissertation document is also comparable with the findings of Langella et al. (2003), as the temperature levels analyzed exhibit Type-2 behaviour (Ciosek and Luk, 2017b). 


\subsubsection{Heavy Metallic Ion Pre-Treatment Trends}

Table 4.7 provides the HMI uptake at 180 minutes $\left(\mathrm{q}_{180}\right.$ in meq/g) of contact for nonheated and heat-pre-treated zeolite in the triple-[T] and multi-[M] component systems. With each heat pre-treatment level, the same trend is maintained among the various component systems. Once again, for all operation parameters investigated in this study, zeolite has the highest affinity and favoured uptake for $\mathrm{Pb}^{2+}$ ion (Ouki and Kavannagh, 1997; Inglezakis et al., 2002, 2003, 2004; Sprynskyy et al., 2006; Ciosek and Luk, 2017a) followed by $\mathrm{Fe}^{2+}$ and $\mathrm{Cu}^{2+}$, with a lower affinity for $\mathrm{Zn}^{2+}$ then $\mathrm{Ni}^{2+}$. A significant loss in crystallinity and hence catalytic activity are common with this pre-treatment process (Akkoca et al., 2013). Dehydration temperature as well as micropore volume and transitional porosity development are directly proportional (Kostandyan et al., 1982). It is important to increase surface area, porosity and sorption capacities of natural zeolites without crystallinity loss (Akkoca et al., 2013). The percent removal of the $\mathrm{Pb}^{2+}$ ion in $[\mathrm{M}]$ is $94.0 \%$, while only $56.7 \%$ in [M-600]. The percent removal of the total HMIs reduces from 16.5 to $3.68 \%$ going from non-heat-pre-treated to $600^{\circ} \mathrm{C}$ exposure. This demonstrates the extreme temperature effects on the zeolite's sorption capacity to the HMIs of interest.

Table 4.7 HMI Uptake (meq/g) by Heat Pre-Treatment Level

\begin{tabular}{ccccccccc}
\hline Heat Level & \multicolumn{2}{c}{ Non-Heated } & \multicolumn{2}{c}{$\mathbf{2 0 0}^{\mathbf{0}} \mathbf{C}$} & \multicolumn{2}{c}{$\mathbf{4 0 0}^{\mathbf{C}}$} & \multicolumn{2}{c}{$\mathbf{6 0 0}^{\mathbf{0}} \mathbf{C}$} \\
\hline System & {$[\mathrm{T}]$} & {$[\mathrm{M}]$} & {$[\mathrm{T}]$} & {$[\mathrm{M}]$} & {$[\mathrm{T}]$} & {$[\mathrm{M}]$} & {$[\mathrm{T}]$} & {$[\mathrm{M}]$} \\
\hline $\mathbf{H M I}$ & & & & & & & & \\
\hline $\mathrm{Pb}^{2+}$ & 0.075 & 0.047 & 0.073 & 0.047 & 0.057 & 0.040 & 0.038 & 0.028 \\
\hline $\mathrm{Cu}^{2+}$ & 0.016 & 0.011 & 0.015 & 0.011 & 0.008 & 0.006 & 0.006 & 0.003 \\
\hline $\mathrm{Fe}^{3+}$ & 0.041 & 0.028 & 0.036 & 0.025 & 0.024 & 0.017 & 0.026 & 0.017 \\
\hline $\mathrm{Ni}^{2+}$ & - & 0.005 & - & 0.004 & - & 0.004 & - & 0.003 \\
\hline $\mathrm{Zn}^{2+}$ & - & 0.008 & - & 0.008 & - & 0.004 & - & 0.002 \\
\hline TOTAL HMI & $\mathbf{0 . 1 3 2 0}$ & $\mathbf{0 . 0 9 8 6}$ & $\mathbf{0 . 1 2 3 9}$ & $\mathbf{0 . 0 9 3 7}$ & $\mathbf{0 . 0 8 8 2}$ & $\mathbf{0 . 0 7 1 2}$ & $\mathbf{0 . 0 7 0 3}$ & $\mathbf{0 . 0 5 2 3}$ \\
\hline
\end{tabular}

Figure 4.5 displays the effects of each heat pre-treatment level, with respect to the percentage of non-heated zeolite uptake. Evidently, the presence of each HMI in solution impacts the uptake of the other; as seen by the interference of the $\mathrm{Ni}^{2+}$ and $\mathrm{Zn}^{2+}$ ions to the [M] component system uptake of the $\mathrm{Cu}^{2+}, \mathrm{Fe}^{3+}$, and $\mathrm{Pb}^{2+}$ ions in the [T] system. Comparing [T] to [M], the uptake of the $\mathrm{Pb}^{2+}$ ion is reduced by $36.2,30.4$ and $25.9 \%$ at the heat pre-treatment levels of 200,400 , and $600^{\circ} \mathrm{C}$, respectively, and $37.5 \%$ without heat pre-treatment. When heatpre-treated to $600^{\circ} \mathrm{C}$, the total HMI uptake is reduced by approximately $47.0 \%$ in both systems. 


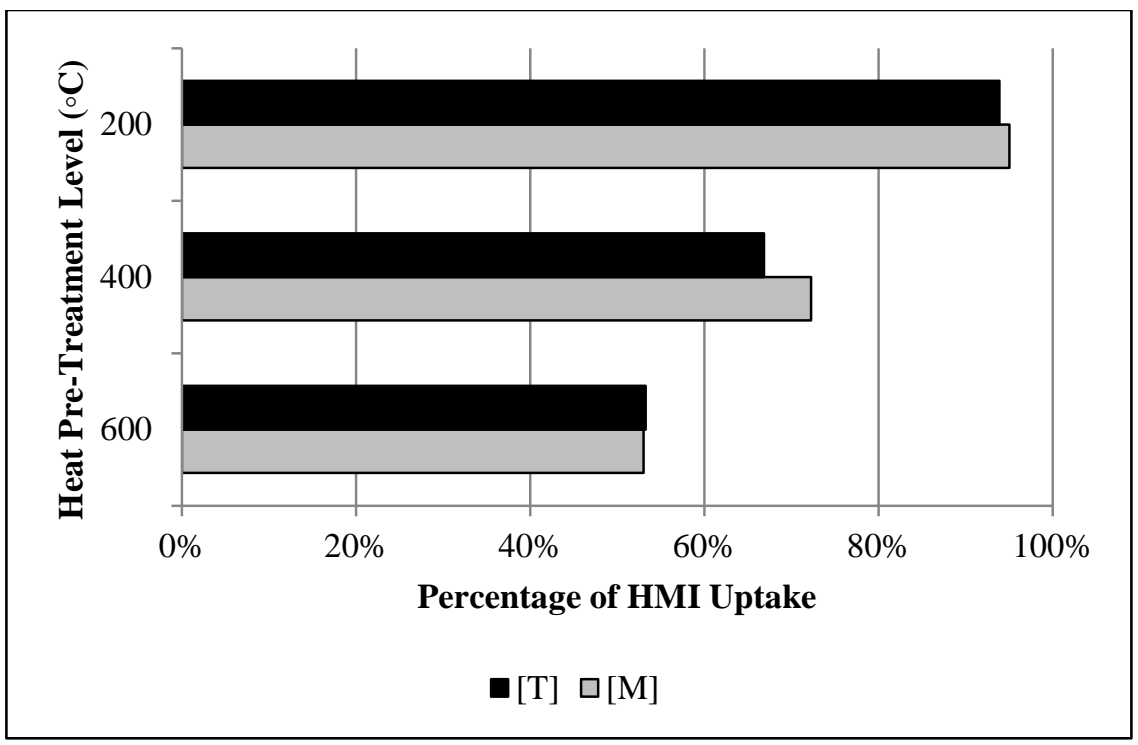

Figure 4.5 Percentage of Non-Heated Level Total HMI Uptake

Figure 4.6 displays the total HMI uptake over the 3-hour contact period for each heat pretreatment level, for the [T] (4.6.a) and [M] (4.6.b) component systems, respectively. This study is consistent with the three distinct stages discussed by Sprynskyy et al. (2006). As expected, there is a slightly greater uptake in the [T] over time; attributed to the interference of the additional two HMIs in the $[\mathrm{M}]$. The rate of uptake for both component systems is not significantly affected by the $200^{\circ} \mathrm{C}$ heat exposure. The first 45 -minute period is very similar for [M-200], compared to the non-heated. Consistent with the findings of Motsi et al. (2009), a substantial reduction of HMI uptake occurs at the $400^{\circ} \mathrm{C}$ threshold. 


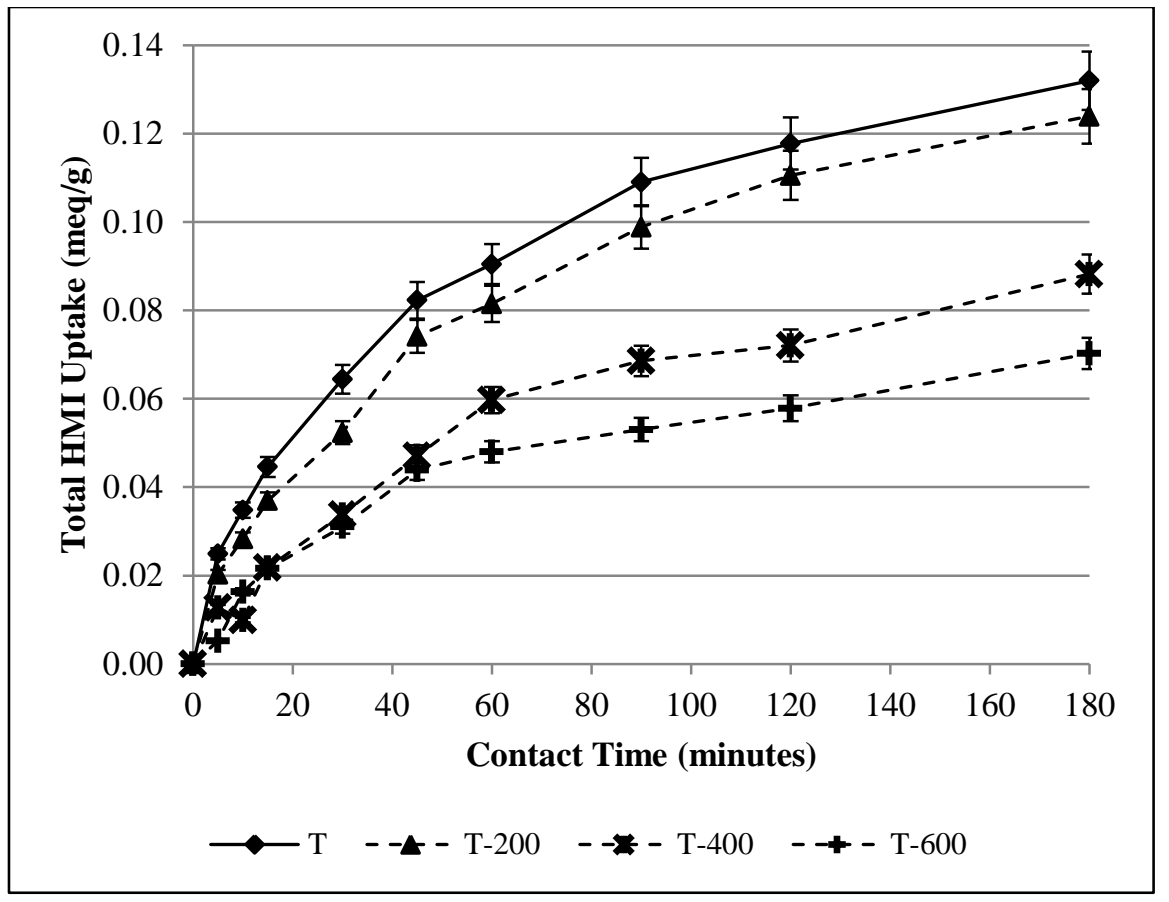

a. Triple-Component System

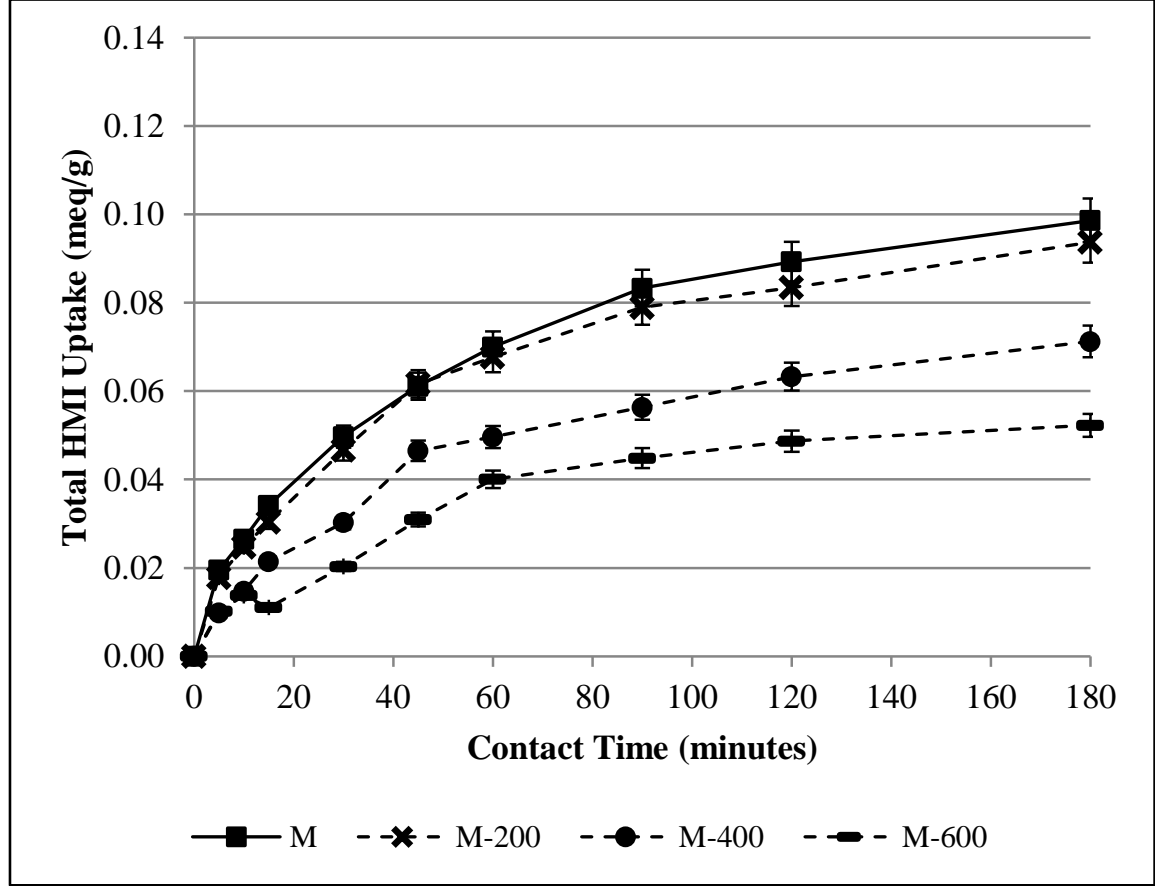

b. Multi-Component System

Figure 4.6 Heat Pre-Treatment Variation of Total HMI Uptake over Time (adapted from Ciosek and Luk, 2017b) 


\subsubsection{Qualitative Pre-Treatment Trends}

Once the cleaning cycle of the raw zeolite sample was complete, the as-received pale green colour was sustained. Following the progressive heat pre-treatment exposure, this colour transitioned to a pink, pale pink, then light brown colour (Ciosek and Luk, 2017b). Figure 4.7 provides SEM images obtained by the high-resolution microscope, taken at $\times 5000$ magnification (5 $\mu \mathrm{m}$ scale bar). Subtle physical changes of the surface structure are observed when comparing the raw granules (shown in 4.7a) to those exhibited to the cleaning cycle (shown in 4.7b). The images of the zeolite exposed to heat pre-treatment of 200,400 , and $600^{\circ} \mathrm{C}$ are shown in $4.7 \mathrm{c}$, $4.7 \mathrm{~d}$, and $4.7 \mathrm{e}$, respectively. To point out once again, a substantial reduction of HMI uptake occurs within the $400-600^{\circ} \mathrm{C}$ temperature range of this study (Motsi et al., 2009). The non-heated uptake in $[\mathrm{T}]$ and $[\mathrm{M}]$ is achieved by 93.9 and $95.0 \%$ in [T-200] and [M-200], respectively. This is qualitatively observed in Figures $4.7 \mathrm{~b}$ and $4.7 \mathrm{c}$, with the visual similarity. The 400 and $600^{\circ} \mathrm{C}$ exposures are not as texturally complex in Figures $4.7 \mathrm{~d}$ and $4.7 \mathrm{e}$, respectively.

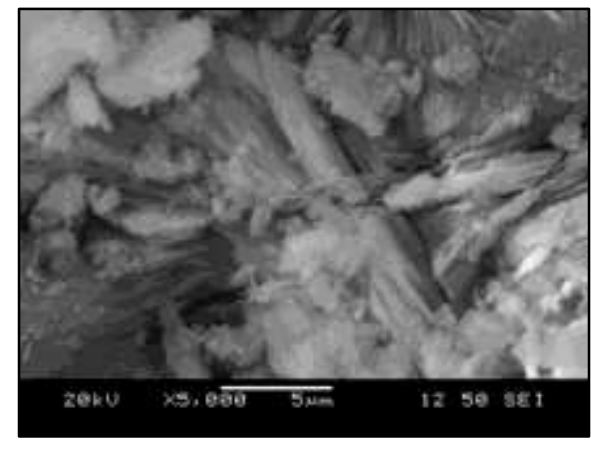

a. Natural Zeolite - As Received

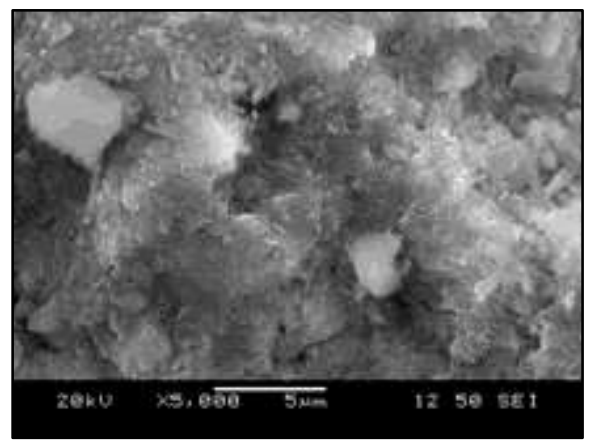

c. Heat Pre-treated at $200^{\circ} \mathrm{C}$

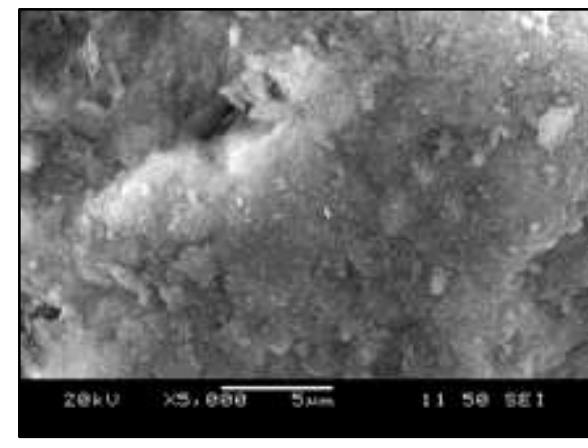

d. Heat Pre-treated at $400^{\circ} \mathrm{C}$

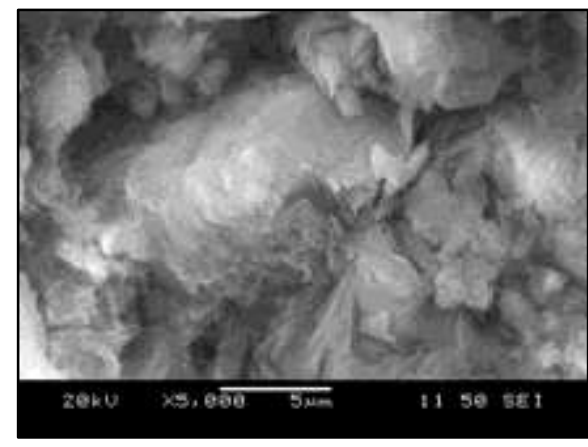

b. Cleaning Cycle

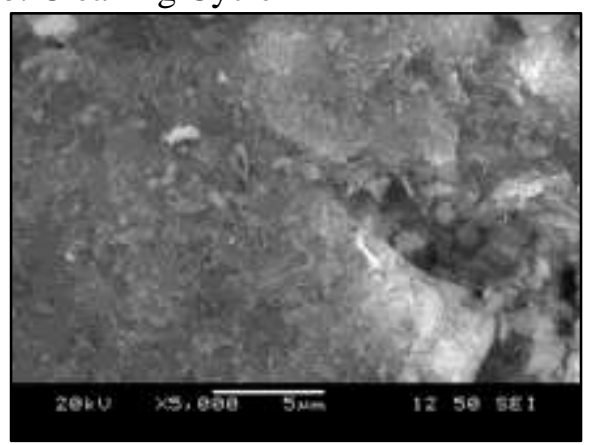

e. Heat Pre-treated at $600^{\circ} \mathrm{C}$

Figure 4.7 SEM Images of Natural Zeolite Exposure to Heat-Pre-treatment (adapted from Ciosek and Luk, 2017b) 
As seen in Table 4.7, the sorption capacity is significantly compromised at the 400 and $600^{\circ} \mathrm{C}$ exposures; which is supported by the lack of textural complexity in Figures $4.7 \mathrm{~d}$ and $4.7 \mathrm{e}$, respectively. It is visually evident that the raw sample possesses textured granularity and significant detail, which is subsequently diminished with heat-pre-treatment towards the intergranular spaces and mineral crevasses. This provides additional knowledge into how the structure of the zeolite mineral has been modified. However, the process of dehydration requires a considerable amount of energy, which practically outweighs the interest to 'activate' the structure of the mineral sample. Both the quantitative and qualitative observations demonstrate that there is no economic benefit to the hydrothermal pre-treatment of the zeolite mineral, under the testing conditions.

\subsubsection{Acidity Observations}

It is important to note that the $\mathrm{pH}$ level of every sample (stock solution and sorbent-solution contact) was measured for all operating parameters investigated; utilizing the accumet Basic AB15 pH Meter (Fisher Scientific; CAT No. 13636 AB15). This was conducted after batchmode contact, and before dilution (following 24 hour storage) preparation for calibration. The set-up of the initial stock pH was kept consistent throughout all experiments, with an average of 1.85 measured. The average maximum and minimum values between the batch bottle and stored supernatant samples are summarized in Table $\mathbf{4 . 8}$.

Table 4.8 The Average $\mathrm{pH}$ Level Data

\begin{tabular}{ccc}
\hline & \multicolumn{2}{c}{ pH Sample } \\
\hline Observation & Batch Contact & Storage Filter \\
\hline Maximum & 2.18 & 2.14 \\
\hline Minimum & 1.90 & 1.89 \\
\hline
\end{tabular}

This data is based on a dosage 40 ratio $(4 \mathrm{~g} / 100 \mathrm{~mL})$; excluding the $\mathrm{pH}$ observations for the dosage $80(8 \mathrm{~g} / 100 \mathrm{~mL})$ parameter. At 45 minutes of contact for all HMIs at dosage 80 conditions, the effluent becomes more basic to reach a maximum of just above 2.20. Overall, the samples collected show an average pH maximum and minimum of 2.39 and 1.91, respectively. This level is still quite acidic, and brings attention to the fact that the $\mathrm{H}^{+}$ions are in competition with the HMI of interest. The doubled dosage provides greater active sites availability for sorption to occur. The decision to proceed with particle size $\mathrm{D}\left(\mathrm{d}_{\mathrm{p}, \mathrm{D}}\right)$ as the controlled parameter moving forward in the analysis is once again justified, given that the $\mathrm{pH}$ was consistent throughout this study. 


\subsection{Conclusions}

The removal efficiency of heavy metallic ions by natural zeolite increases by:

- Decreasing particle size and influent concentration;

- Increasing dosage level, contact time and set-temperature, and by;

- Maintaining the heat-pre-treatment level below the activation threshold.

The efficiency is improved depending on the specific metal and the parameter observed, it can be qualitatively speculated that other heavy metals would be equally influenced (Ouki and Kavannagh, 1997). The removal efficiency order (or selectivity series) is consistent for all examined experimental conditions: $\mathrm{Pb}^{2+}>>\mathrm{Fe}^{3+}>\mathrm{Cu}^{2+}>\mathrm{Zn}^{2+}>\mathrm{Ni}^{2+}$ (Ciosek and Luk, 2017a; 2017b).

The sorption uptake of HMIs by natural zeolite is complex, due to the aqueous chemistry of the elements and the nature of the sorbent mineral (Oter and Akcay, 2007). However, this research provides a greater understanding into how the presence of multiple metallic ions and various operative parameters impact the overall removal efficiency under the testing conditions, and indicates how the sorption properties of zeolite influence the overall selectivity trends. This is a significant contribution to the current knowledge-base, and how these parameters impact the removal capacity of natural mineral in batch-mode configuration.

\subsection{Future Works}

This study demonstrates that the zeolite mineral exhibits the greatest preference towards the lead $\left(\mathrm{Pb}^{2+}\right)$ ion, regardless of how the operation parameters or conditions are altered. This prompts the interest to further investigate how the other HMIs influence the removal of $\mathrm{Pb}^{2+}$ when combined, and to further develop a better understanding of the interactive trends associated in the sorption process. Given that lead is considered a designated substance of toxic priority, the focus of this next chapter is of significance to the industry and the peripheral environment at risk. 


\title{
5. LEAD REMOVAL CAPACITY AND SELECTIVITY Lead Removal from Mine Tailings with Multiple Metallic Ions International Journal of Water and Wastewater Treatment 2017. 3(1). 1-9. doi:10.16966/2381-5299.134.
}

Fundamental components of this chapter were also presented as a paper at the WEAO2016 Technical Symposium ('Industrial Treatment A' Session); referenced in Appendix C of the Dissertation document.

\begin{abstract}
This study investigates the ionic effects of $\mathrm{Cu}^{2+}, \mathrm{Fe}^{3+}, \mathrm{Ni}^{2+}$ and $\mathrm{Zn}^{2+}$ on the sorption of the target metal ion $\mathrm{Pb}^{2+}$ by natural zeolite in the form of clinoptilolite. The batch testing configuration consisted of a synthetic nitrate salt solution maintained at $10 \mathrm{meq} / \mathrm{L}$ system total concentration, acidified to a $\mathrm{pH}$ of 2 by $\mathrm{HNO}_{3}$ acid, with an adsorbent dosage of $40 \mathrm{~g} / \mathrm{L}$. The aqueous solution was agitated for a contact period of 5 to 180 minutes by means of a tripleeccentric drive orbital shaker operating at $400 \mathrm{r} / \mathrm{min}$. The objective is to determine the overall sorption of the target metal lead and the selectivity of clinoptilolite to the various heavy metallic ions (HMIs) combined in aqueous phase. Elemental analysis concludes that after 3 contact hours, the lead ion is removed by $76.8 \%$ in the single-lead system. In the dual-lead system, $\mathrm{Pb}^{2+}$ is removed by $88.3,79.5,89.1$, and $88.5 \%$ when combined with copper, iron, nickel, and zinc, respectively. Finally, $\mathrm{Pb}^{2+}$ is removed by 90.2 and $94.0 \%$ in the triple-(lead-copper-iron) and multi-component systems, respectively. As the HMIs are introduced to the system with a 10 meq/L total concentration, the uptake of the $\mathrm{Pb}^{2+}$ ion in the single-system of $7.68 \mathrm{meq} / \mathrm{L}$ is decreased by $75.6 \%$ to $1.88 \mathrm{meq} / \mathrm{L}$ in the multi-component system. Overall, the total HMIs removed decreases from $7.68 \mathrm{meq} / \mathrm{L}$ in the single-system to $3.95 \mathrm{meq} / \mathrm{L}$ with the subsequent addition of ions in the multi-component system.
\end{abstract}




\subsection{Introduction}

This chapter focuses on the heavy metallic ion lead $\left(\mathrm{Pb}^{2+}\right)$. This particular HMI is known as a designated substance, with carcinogenic consequences that affect every organ and system in the human body (OHSA, 2014). Based on the Canada-Wide Survey of Acid Mine Drainage Characteristics (Wilson, 1994), the annual-seasonal average levels for lead $\left(\mathrm{Pb}^{2+}\right)$ is $0.50 \mathrm{mg} / \mathrm{L}$, exceeding the mean monthly effluent limit of $0.20 \mathrm{mg} / \mathrm{L}$ (CMJ, 2014) by $150 \%$. The Occupational Health and Safety Act (OHSA, 2014) has identified lead as a designated substance of acute toxic priority (Kragovic et al., 2013) that poses a great risk to our ecosystem, with carcinogenic consequences that affect every organ and system in the human body (OHSA, 2014). As discussed in Chapter 4, the effects of operating conditions investigated in the first phase of the research project demonstrate that regardless of condition, the trend among the five (5) HMIs selected is consistent. Compared to the other HMIs, $\mathrm{Pb}^{2+}$ is superior in uptake as it is not composed of complex, hydrated compounds and thereby demonstrates a significantly higher selectivity by clinoptilolite (Ouki and Kavannagh, 1997; Inglezakis et al., 2003). This particular HMI was chosen to further the understanding of zeolite's affinity and selectivity, and how the other HMIs affect the removal uptake when combined.

\subsection{Materials and Methods}

Refer to Chapter 3 for the consolidated experimental methodology; sorbent material, sorbate solution, and analytical procedure. All analyses were conducted in batch-mode, creating the synthetic simple solute HMI solution in the various component system combinations at a 4-g sorbent mass to $100-\mathrm{mL}$ sorbate volume dosage.

\subsection{Quality Control (QC) Protocol}

Several measures were applied to ensure quality control (QC) of the experimental investigations carried out in this study. During the preliminary stages of investigation, all of the batch sorption experiments were conducted twice. Filter separation was investigated with 5 and 180 minutes effluent samples, with a first then second filter pass. Only a maximum of $1.50 \%$ difference among the filtered sets was measured, and supports that the agitation method in triple- 
eccentric drive for adsorbent-adsorbate contact is effective. These preliminary findings demonstrated that the protocol established is repeatable and moving forward, all other tests were run once per sample. Furthermore, each sample concentration was read in triplicates as set in the ICP-AES system software for validation purposes. Quality control checks were applied to both the calibration standards and the sorption results.

Table 5.1 summarizes the QC checks conducted on the calibration standard of $50 \mathrm{mg} / \mathrm{L}$ for the various component systems. The \%-RSD values are considerably lower than the $3 \%$ targeted limit, and a majority of detected concentrations fall well within $5 \%$ of the $50 \mathrm{mg} / \mathrm{L}$ theoretical value.

Table 5.1 Calibration Standard 50 QC Check of Various Component Systems

\begin{tabular}{ccccc}
\hline Sample ID & Analyte & $\begin{array}{c}\text { \%-RSD } \\
\text { (Corr Int) }\end{array}$ & $\begin{array}{c}\text { Triplicate Mean } \\
\text { (Calib) }(\mathbf{m g} / \mathbf{L})\end{array}$ & Corr Coef \\
\hline \multirow{2}{*}{ SINGLE } & $\mathrm{Cu} 327.393$ & 0.81 & 52.98 & 0.999729 \\
{$[\mathrm{Fe} 238.204$} & 0.90 & 53.91 & 0.999745 \\
& $\mathrm{Ni} 231.604$ & 1.06 & 54.28 & 0.999731 \\
& $\mathrm{~Pb} 220.353$ & 0.40 & 53.87 & 0.999710 \\
& $\mathrm{Zn} 206.200$ & 1.36 & 54.69 & 0.999675 \\
\hline & $\mathrm{Cu} 327.393$ & 0.13 & 50.78 & 0.999867 \\
DUAL & $\mathrm{Fe} 238.204$ & 0.19 & 51.32 & 0.999909 \\
{$[\mathrm{D}]$} & $\mathrm{Ni} 231.604$ & 0.18 & 51.18 & 0.999916 \\
& $\mathrm{~Pb} 220.353$ & 0.58 & 51.43 & 0.999838 \\
& $\mathrm{Zn} 206.200$ & 0.19 & 50.66 & 0.999960 \\
\hline & $\mathrm{Cu} 327.393$ & 0.32 & 52.12 & 0.999966 \\
TRIPLE [T] & $\mathrm{Fe} 238.204$ & 0.45 & 52.50 & 0.999953 \\
MULTI [M] & $\mathrm{Ni} 231.604$ & 0.61 & 52.48 & 0.999948 \\
& $\mathrm{~Pb} 220.353$ & 0.75 & 52.06 & 0.999900 \\
& $\mathrm{Zn} 206.200$ & 0.96 & 52.01 & 0.999968 \\
\hline
\end{tabular}




\subsection{Analysis}

\subsubsection{Quantitative Observations - Acidity and Heavy Metallic Ion Levels}

Table 5.2 provides a summary of the sample preparations involved for elemental analysis. The 'Sample ID' column outlines each component system, and their respective influent stock (S) and contact times, which ranged from 5 to 180 minutes. The number of 50\% dilutions are indicated in the column 'DIL', which is required to keep all concentrations within the calibration range of $0-100 \mathrm{mg} / \mathrm{L}$. Based on the established sorbent-to-sorbate ratio of $4 \mathrm{~g}$ to $100 \mathrm{~mL}$, the measured 'Zeolite Mass' is provided for each sample. Finally, the 'pH Level' verification results are provided for both directly from sample on the day of zeolite exposure, and the day after of the filtered sample. Following sorbate-sorbent contact, the $\mathrm{pH}$ is observed to be steady throughout the ion-exchange process, with a maximum of $0.20 \mathrm{pH}$ difference after 3 contact hours. The $\mathrm{pH}$ of the prepared influent stock and filtered effluent are also observed to be consistent during refrigerated storage prior to dilution preparation for elemental analysis.

The quantitative results of the various systems are summarized in Table 5.3. The primary wavelengths, given in Column 1, to the ICP-AES model were applied for all five metal ions to generate the Relative Standard Deviation (\%-RSD) as shown in Column 3. Triplicate readings and their mean concentrations in calibration units are presented in $\mathrm{mg} / \mathrm{L}$, and given in Column 4. Based on the dilution factors presented in Table 5.2, the corresponding HMI concentrations and percent removal after 3 hours were calculated and provided in Column 5 and 6, respectively. Overall, the \%-RSD values are low and all within $\leq 3 \%$, which is indicative that the data are accurate and the experimental replicates are reliable (Perkin Elmer Inc., 2010). A significant observation is made for that of the target metal ion. The overall removal of the lead ion is highest compared to the four other HMI investigated. In addition, this removal level is consistently high regardless of the component system. This is a first glimpse in this study of zeolite's preference for lead, and the overall impact of the presence of the other ions. 
Table 5.2 Sample Preparation with pH Level Verification

\begin{tabular}{|c|c|c|c|c|c|c|c|c|c|c|c|c|c|c|}
\hline \multirow{2}{*}{ DIL } & \multirow{2}{*}{$\begin{array}{c}\text { Sample } \\
\text { ID }\end{array}$} & \multirow{2}{*}{$\begin{array}{c}\text { Zeolite } \\
\text { Mass }\end{array}$} & \multicolumn{2}{|c|}{ pH Level } & \multirow{2}{*}{ DIL } & \multirow{2}{*}{$\begin{array}{c}\text { Sample } \\
\text { ID }\end{array}$} & \multirow{2}{*}{$\begin{array}{c}\text { Zeolite } \\
\text { Mass }\end{array}$} & \multicolumn{2}{|c|}{ pH Level } & \multirow{2}{*}{ DIL } & \multirow{2}{*}{$\begin{array}{c}\text { Sample } \\
\text { ID }\end{array}$} & \multirow{2}{*}{$\begin{array}{c}\text { Zeolite } \\
\text { Mass }\end{array}$} & \multicolumn{2}{|c|}{ pH Level } \\
\hline & & & Expose & Filter & & & & Expose & Filter & & & & Expose & Filter \\
\hline \multirow{5}{*}{4} & {$[\mathrm{P}]-\mathrm{S}$} & --- & -- & 1.82 & \multirow{5}{*}{3} & [D-PC]-S & --- & -- & 1.87 & \multirow{5}{*}{3} & [D-PN]-S & --- & -- & 1.70 \\
\hline & {$[\mathrm{P}] 5$} & 4.0018 & 1.92 & 1.84 & & [D-PC]5 & 4.0049 & 1.94 & 1.89 & & [D-PN]5 & 4.0043 & 1.90 & 1.84 \\
\hline & {$[\mathrm{P}] 10$} & 4.0013 & 1.94 & 1.88 & & [D-PC]10 & 4.0063 & 1.98 & 1.91 & & {$[\mathrm{D}-\mathrm{PN}] 10$} & 4.0070 & 1.98 & 1.93 \\
\hline & {$[\mathrm{P}] 15$} & 4.0088 & 1.95 & 1.93 & & [D-PC] 15 & 4.0048 & 2.01 & 1.92 & & [D-PN]15 & 4.0044 & 2.00 & 1.97 \\
\hline & {$[\mathrm{P}] 30$} & 4.0042 & 2.02 & 1.95 & & {$[\mathrm{D}-\mathrm{PC}] 30$} & 4.0072 & 2.03 & 1.97 & & {$[\mathrm{D}-\mathrm{PN}] 30$} & 4.0036 & 2.04 & 2.00 \\
\hline \multirow{5}{*}{3} & {$[\mathrm{P}] 45$} & 4.0041 & 2.03 & 1.99 & \multirow{5}{*}{2} & {$[\mathrm{D}-\mathrm{PC}] 45$} & 4.0045 & 2.08 & 2.05 & \multirow{5}{*}{2} & {$[\mathrm{D}-\mathrm{PN}] 45$} & 4.0025 & 2.09 & 1.99 \\
\hline & {$[\mathrm{P}] 60$} & 4.0084 & 2.04 & 1.98 & & {$[\mathrm{D}-\mathrm{PC}] 60$} & 4.0069 & 2.12 & 2.09 & & [D-PN]60 & 4.0072 & 2.14 & 2.05 \\
\hline & {$[\mathrm{P}] 90$} & 4.0055 & 2.07 & 2.01 & & [D-PC]90 & 4.0041 & 2.19 & 2.13 & & [D-PN]90 & 4.0068 & 2.10 & 2.04 \\
\hline & {$[\mathrm{P}] 120$} & 4.0061 & 2.09 & 2.01 & & [D-PC]120 & 4.0067 & 2.15 & 2.13 & & [D-PN]120 & 4.0084 & 2.17 & 2.10 \\
\hline & {$[\mathrm{P}] 180$} & 4.0025 & 2.10 & 2.04 & & [D-PC] 180 & 4.0062 & 2.18 & 2.15 & & [D-PN]180 & 4.0075 & 2.18 & 2.11 \\
\hline \multirow{5}{*}{2} & {$[\mathrm{~T}]-\mathrm{S}$} & --- & --- & 1.88 & \multirow{5}{*}{3} & [D-PF]-S & --- & --- & 1.79 & \multirow{5}{*}{3} & [D-PZ]-S & --- & --- & 1.83 \\
\hline & {$[\mathrm{T}] 5$} & 4.0083 & 1.97 & 1.90 & & [D-PF]5 & 4.0061 & 1.89 & 1.82 & & [D-PZ]5 & 4.0063 & 1.85 & 1.85 \\
\hline & {$[\mathrm{T}] 10$} & 4.0090 & 2.01 & 1.94 & & [D-PF]10 & 4.0076 & 1.92 & 1.84 & & [D-PZ]10 & 4.0088 & 1.94 & 1.94 \\
\hline & {$[\mathrm{T}] 15$} & 4.0073 & 2.03 & 1.92 & & [D-PF] 15 & 4.0088 & 1.96 & 1.93 & & [D-PZ]15 & 4.0027 & 1.99 & 1.97 \\
\hline & {$[\mathrm{T}] 30$} & 4.0060 & 2.07 & 1.96 & & {$[\mathrm{D}-\mathrm{PF}] 30$} & 4.0074 & 2.01 & 1.98 & & [D-PZ]30 & 4.0068 & 2.02 & 2.01 \\
\hline \multirow{5}{*}{1} & {$[\mathrm{~T}] 45$} & 4.0026 & 2.11 & 2.01 & \multirow{5}{*}{2} & [D-PF]45 & 4.0012 & 1.99 & 1.99 & \multirow{5}{*}{2} & [D-PZ]45 & 4.0081 & 2.06 & 2.00 \\
\hline & {$[\mathrm{T}] 60$} & 4.0014 & 2.11 & 2.03 & & [D-PF]60 & 4.0059 & 2.06 & 2.06 & & [D-PZ]60 & 4.0082 & 2.14 & 2.06 \\
\hline & {$[\mathrm{T}] 90$} & 4.0005 & 2.17 & 2.09 & & [D-PF]90 & 4.0077 & 2.10 & 2.08 & & [D-PZ]90 & 4.0045 & 2.10 & 2.04 \\
\hline & {$[\mathrm{T}] 120$} & 4.0045 & 2.15 & 2.08 & & {$[\mathrm{D}-\mathrm{PF}] 120$} & 4.0068 & 2.11 & 2.12 & & [D-PZ]120 & 4.0038 & 2.15 & 2.06 \\
\hline & {$[\mathrm{T}] 180$} & 4.0073 & 2.19 & 2.08 & & {$[\mathrm{D}-\mathrm{PF}] 180$} & 4.0075 & 2.14 & 2.11 & & [D-PZ]180 & 4.0038 & 2.17 & 2.10 \\
\hline \multirow{5}{*}{1} & {$[\mathrm{M}]-\mathrm{S}$} & --- & --- & 1.85 & & & & & & & & & & \\
\hline & {$[\mathrm{M}] 5$} & 4.0050 & 1.93 & 1.90 & & & & & & & & & & \\
\hline & {$[\mathrm{M}] 10$} & 4.0082 & 1.97 & 1.89 & & & & & & & & & & \\
\hline & {$[\mathrm{M}] 15$} & 4.0081 & 2.00 & 1.92 & & & & & & & & & & \\
\hline & {$[\mathrm{M}] 30$} & 4.0064 & 2.05 & 1.99 & & & & & & & & & & \\
\hline \multirow{5}{*}{$\mathbf{0}$} & {$[\mathrm{M}] 45$} & 4.0030 & 2.07 & 2.00 & & & & & & & & & & \\
\hline & {$[\mathrm{M}] 60$} & 4.0071 & 2.09 & 1.93 & & & & & & & & & & \\
\hline & {$[\mathrm{M}] 90$} & 4.0049 & 2.10 & 2.01 & & & & & & & & & & \\
\hline & {$[\mathrm{M}] 120$} & 4.0045 & 2.13 & 2.01 & & & & & & & & & & \\
\hline & {$[\mathrm{M}] 180$} & 4.0076 & 2.17 & 2.04 & & & & & & & & & & \\
\hline
\end{tabular}


Table 5.3 ICP-AES HMI Results of Various Component Systems

\begin{tabular}{|c|c|c|c|c|c|c|c|c|c|c|c|}
\hline \multirow[b]{3}{*}{ Analyte } & 2 & 3 & 4 & 5 & 6 & 1 & 2 & 3 & 4 & 5 & 6 \\
\hline & \multirow{2}{*}{$\begin{array}{c}\text { Sample } \\
\text { ID }\end{array}$} & \multirow[b]{2}{*}{$\begin{array}{c}\% \text {-RSD } \\
\text { (Corr Int) }\end{array}$} & \multicolumn{2}{|c|}{ Concentration (mg/L) } & \multirow[b]{2}{*}{$\begin{array}{c}\% \\
\text { Removal }\end{array}$} & \multirow[b]{2}{*}{ Analyte } & \multirow[b]{2}{*}{$\begin{array}{c}\text { Sample } \\
\text { ID }\end{array}$} & \multirow[b]{2}{*}{$\begin{array}{c}\% \text {-RSD } \\
\text { (Corr Int) }\end{array}$} & \multicolumn{2}{|c|}{ Concentration (mg/L) } & \multirow{2}{*}{$\begin{array}{c}\% \\
\text { Removal }\end{array}$} \\
\hline & & & $\begin{array}{c}\text { Triplicate } \\
\text { Mean (Calib) }\end{array}$ & DIL & & & & & $\begin{array}{c}\text { Triplicate } \\
\text { Mean (Calib) }\end{array}$ & DIL & \\
\hline $\mathrm{Pb}$ & {$[\mathrm{P}]-\mathrm{S}$} & 0.24 & 75.93 & 1214.81 & \multirow{2}{*}{76.8} & $\mathrm{~Pb}$ & {$[\mathrm{~T}]-\mathrm{S}$} & 1.27 & 100.05 & 400.21 & \multirow{2}{*}{90.2} \\
\hline \multirow[t]{2}{*}{220.353} & {$[\mathrm{P}] 180$} & 0.59 & 35.20 & 281.63 & & 220.353 & {$[\mathrm{~T}] 180$} & 0.21 & 19.66 & 39.32 & \\
\hline & & & & & & $\mathrm{Cu}$ & {$[\mathrm{T}]-\mathrm{S}$} & 1.16 & 33.76 & 135.03 & \multirow{2}{*}{19.0} \\
\hline $\mathrm{Pb}$ & [D-PC]-S & 0.13 & 75.47 & 603.79 & \multirow{2}{*}{88.3} & 327.393 & [T]180 & 1.58 & 54.70 & 109.40 & \\
\hline 220.353 & [D-PC]180 & 0.59 & 17.68 & 70.73 & & $\mathrm{Fe}$ & {$[\mathrm{T}]-\mathrm{S}$} & 0.59 & 17.23 & 68.92 & \multirow{2}{*}{49.5} \\
\hline \multirow{2}{*}{$\begin{array}{c}\mathrm{Cu} \\
327.393 \\
\end{array}$} & [D-PC]-S & 0.11 & 24.18 & 193.44 & \multirow{2}{*}{12.2} & 238.204 & [T]180 & 1.81 & 17.40 & 34.80 & \\
\hline & {$[\mathrm{D}-\mathrm{PC}] 180$} & 0.61 & 42.44 & 169.77 & & & & & & & \\
\hline $\mathrm{Pb}$ & [D-PF]-S & 0.42 & 75.98 & 607.82 & \multirow{2}{*}{79.5} & $\mathrm{~Pb}$ & {$[\mathrm{M}]-\mathrm{S}$} & 0.53 & 117.76 & 235.52 & \multirow{2}{*}{94.0} \\
\hline 220.353 & {$[\mathrm{D}-\mathrm{PF}] 180$} & 0.54 & 31.19 & 124.77 & & 220.353 & {$[\mathrm{M}] 180$} & 0.67 & 14.20 & 14.20 & \\
\hline $\mathrm{Fe}$ & [D-PF]-S & 0.31 & 13.01 & 104.11 & \multirow{2}{*}{37.7} & $\mathrm{Cu}$ & {$[\mathrm{M}]-\mathrm{S}$} & 1.96 & 37.58 & 75.16 & \multirow{2}{*}{21.9} \\
\hline \multirow[t]{2}{*}{238.204} & [D-PF] 180 & 0.45 & 16.21 & 64.82 & & 327.393 & {$[\mathrm{M}] 180$} & 0.99 & 58.74 & 58.74 & \\
\hline & & & & & & $\mathrm{Fe}$ & {$[\mathrm{M}]-\mathrm{S}$} & 2.26 & 20.52 & 41.03 & \multirow{2}{*}{56.2} \\
\hline $\mathrm{Pb}$ & [D-PN]-S & 0.82 & 77.16 & 617.29 & \multirow{2}{*}{89.1} & 238.204 & {$[\mathrm{M}] 180$} & 1.24 & 17.97 & 17.97 & \\
\hline 220.353 & [D-PN]180 & 0.68 & 16.81 & 67.22 & & $\mathrm{Ni}$ & {$[\mathrm{M}]-\mathrm{S}$} & 0.56 & 32.87 & 65.75 & \multirow{2}{*}{9.10} \\
\hline $\mathrm{Ni}$ & [D-PN]-S & 0.86 & 21.05 & 168.37 & \multirow{2}{*}{7.40} & 231.604 & {$[\mathrm{M}] 180$} & 1.48 & 59.76 & 59.76 & \\
\hline 231.604 & [D-PN]180 & 0.12 & 38.99 & 155.94 & & $\mathrm{Zn}$ & {$[\mathrm{M}]-\mathrm{S}$} & 2.23 & 35.99 & 71.98 & \multirow{2}{*}{16.5} \\
\hline & & & & & & 206.200 & {$[\mathrm{M}] 180$} & 1.63 & 60.12 & 60.12 & \\
\hline \multirow{2}{*}{$\begin{array}{c}\mathrm{Pb} \\
220.353 \\
\end{array}$} & [D-PZ]-S & 1.01 & 76.78 & 614.22 & \multirow{2}{*}{88.5} & & & & & & \\
\hline & [D-PZ]180 & 0.50 & 17.68 & 70.72 & & & & & & & \\
\hline \multirow{2}{*}{$\begin{array}{c}\mathrm{Zn} \\
206.200\end{array}$} & [D-PZ]-S & 0.92 & 23.46 & 187.65 & & & & & & & \\
\hline & [D-PZ]180 & 0.83 & 41.75 & 166.98 & 11.0 & & & & & & \\
\hline
\end{tabular}




\subsubsection{Qualitative Observations}

Figure 5.1 displays the SEM images of the natural zeolite taken at $\times 1000$ magnification (10 $\mu \mathrm{m}$ scale bar). The physical changes of the surface structure are illustrated when comparing the raw granules (shown in 5.1a) and those exposed to the cleaning cycle of debris and moisture removal (shown in 5.1b). Once cleaned, the zeolite mineral has greater detailed textured granularity of its inter-granular spaces and mineral crevasses; providing visual evidence that the zeolite has been prepared for the sorption process.

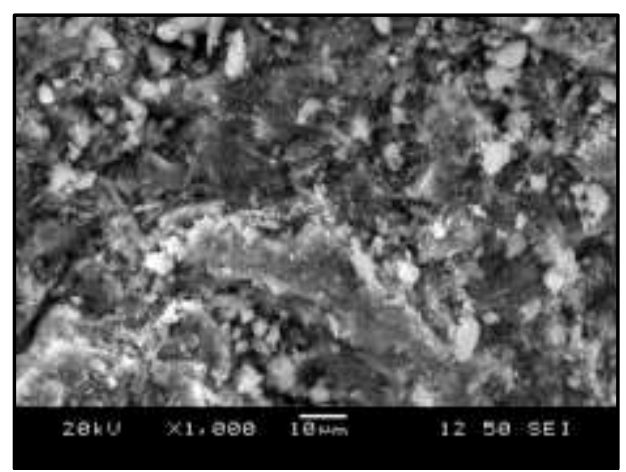

a. Raw Zeolite

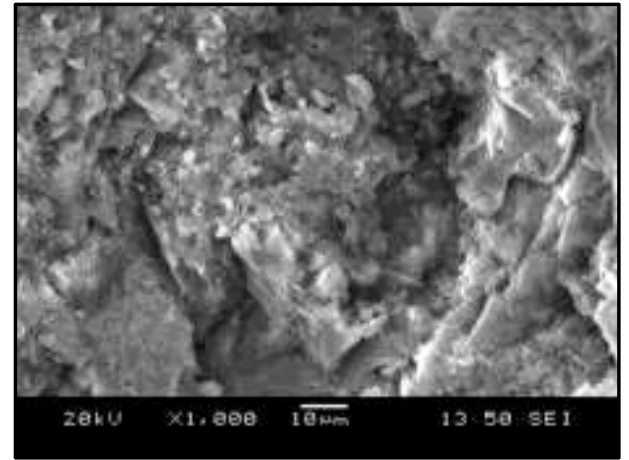

c. $3 \mathrm{hr}$ (M180) Solution Exposure

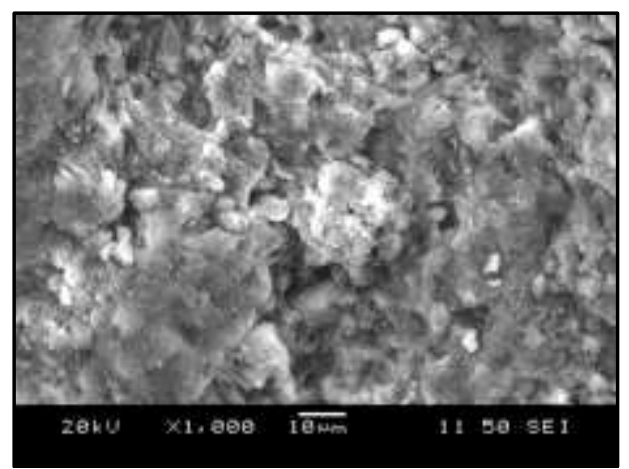

b. Cleaned Zeolite

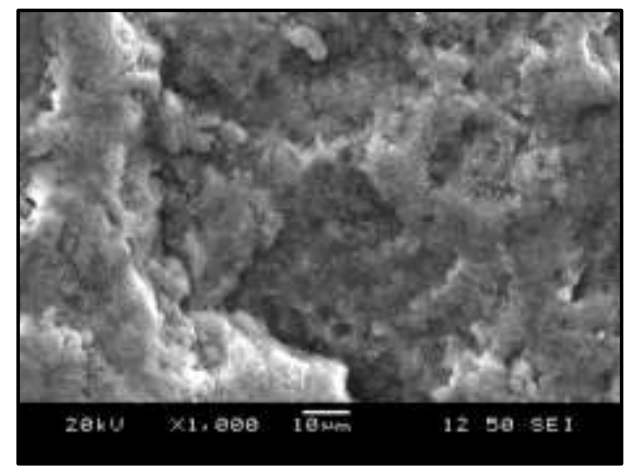

d. $6 \mathrm{hr}$ (M360) Solution Exposure

Figure 5.1 SEM Images of Natural Zeolite Exposure to Multi-Component Solution Over Time (adapted from Ciosek and Luk, 2017b) 
The images of the zeolite exposed to the multi-component solution containing all metals over 3 and 6 hours of contact are shown in Figures 5.1c (M180) and 5.1d (M360), respectively. The spectrum of x-ray energy versus counts were evaluated by EDS to determine the elemental composition of the samples exposed to the multi-component solution, and presented in Figure 5.2 based on a $25 \mu \mathrm{m}$ scale of the samples. As expected, a majority of the zeolite is composed of oxygen, silicon, and aluminum, as well as the co-cations. The presence of lead $(\mathrm{Pb})$ has more than doubled ( 0.6 to $1.7 \mathrm{Wt} \%$ ) with the additional 3 contact hours; further solidifying the strong preference of zeolite towards this particular heavy metal.

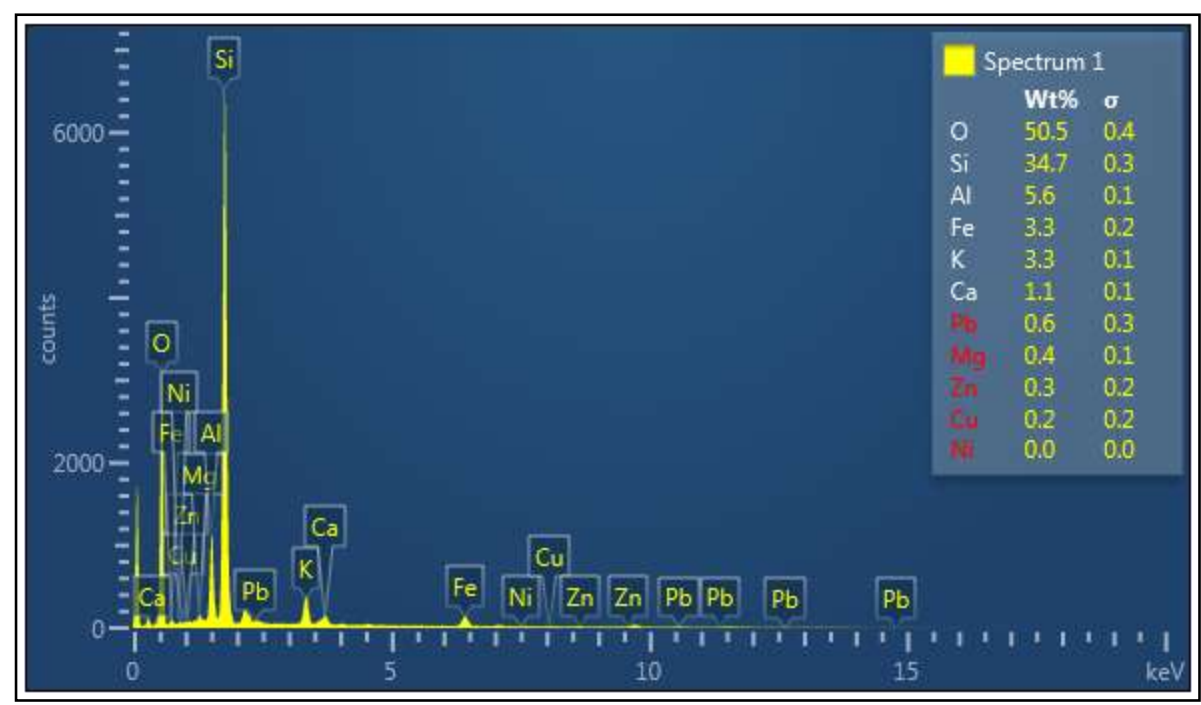

a. $3 \mathrm{hr}$ (M180) Solution Exposure

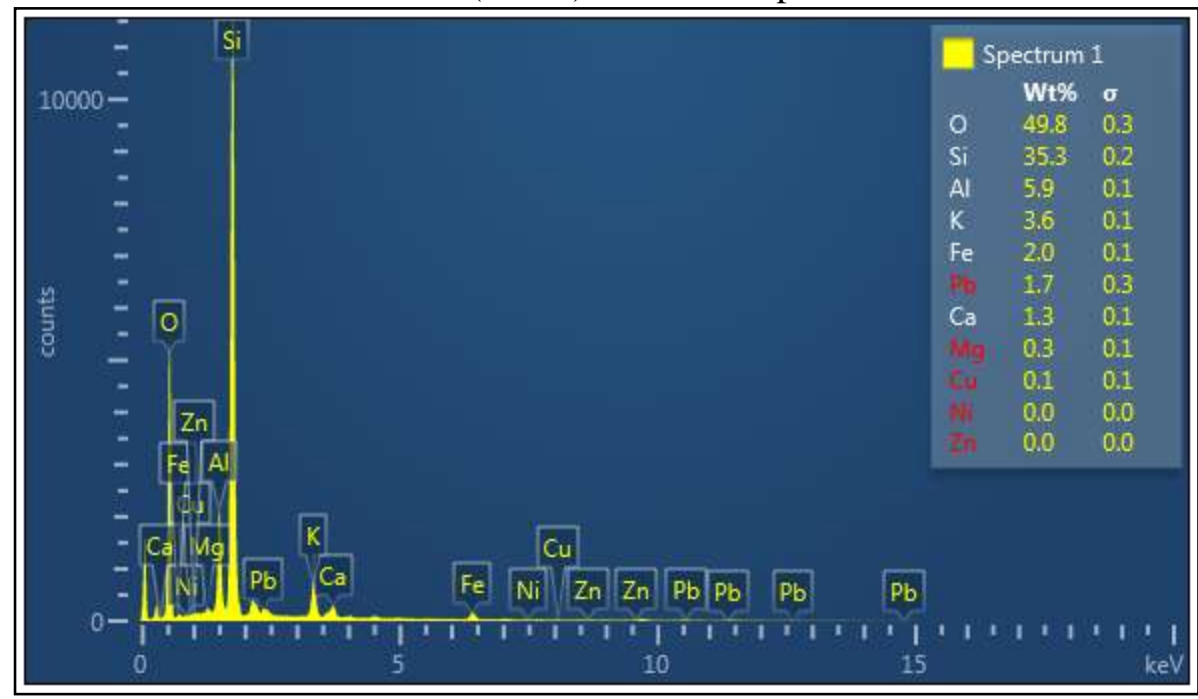

b. $6 \mathrm{hr}$ (M360) Solution Exposure

Figure 5.2 EDS Spectrum of Zeolite Sample Exposure to Multi-Component Solution 


\subsubsection{Percent Removal by Sorption}

Figure 5.3 displays the percent removal trend of lead over time, and how it varies among the various HMI component systems. The elemental analysis results have indicated that after just 3 hours of contact with natural zeolite, the target metal ion lead is removed by $76.8 \%$ in the single-lead system. In the dual-lead system, the lead ion is removed by 88.3, 79.5, 89.1, and 88.5\% when combined with copper [D-PC], iron [D-PF], nickel [D-PN], and zinc [D-PZ], respectively. The targeted lead ion is removed by 90.2 and $94.0 \%$ in the triple-(lead-copper-iron) $[\mathrm{T}]$, and multi-component $[\mathrm{M}]$ systems, respectively. In the multi-component system involving all five metals, the percent removal observed for $\mathrm{Pb}^{2+}, \mathrm{Cu}^{2+}, \mathrm{Fe}^{3+}, \mathrm{Ni}^{2+}$, and $\mathrm{Zn}^{2+}$ are 94.0, 21.9, $56.2,9.10$, and $16.5 \%$, respectively. The corresponding removal trends of each HMI in the [M] system are illustrated in Figure 5.4. Zeolite's preference towards each metal ion is also observed

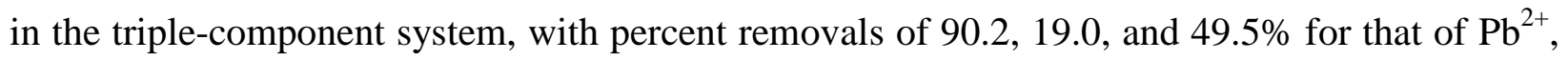
$\mathrm{Cu}^{2+}$, and $\mathrm{Fe}^{3+}$, respectively.

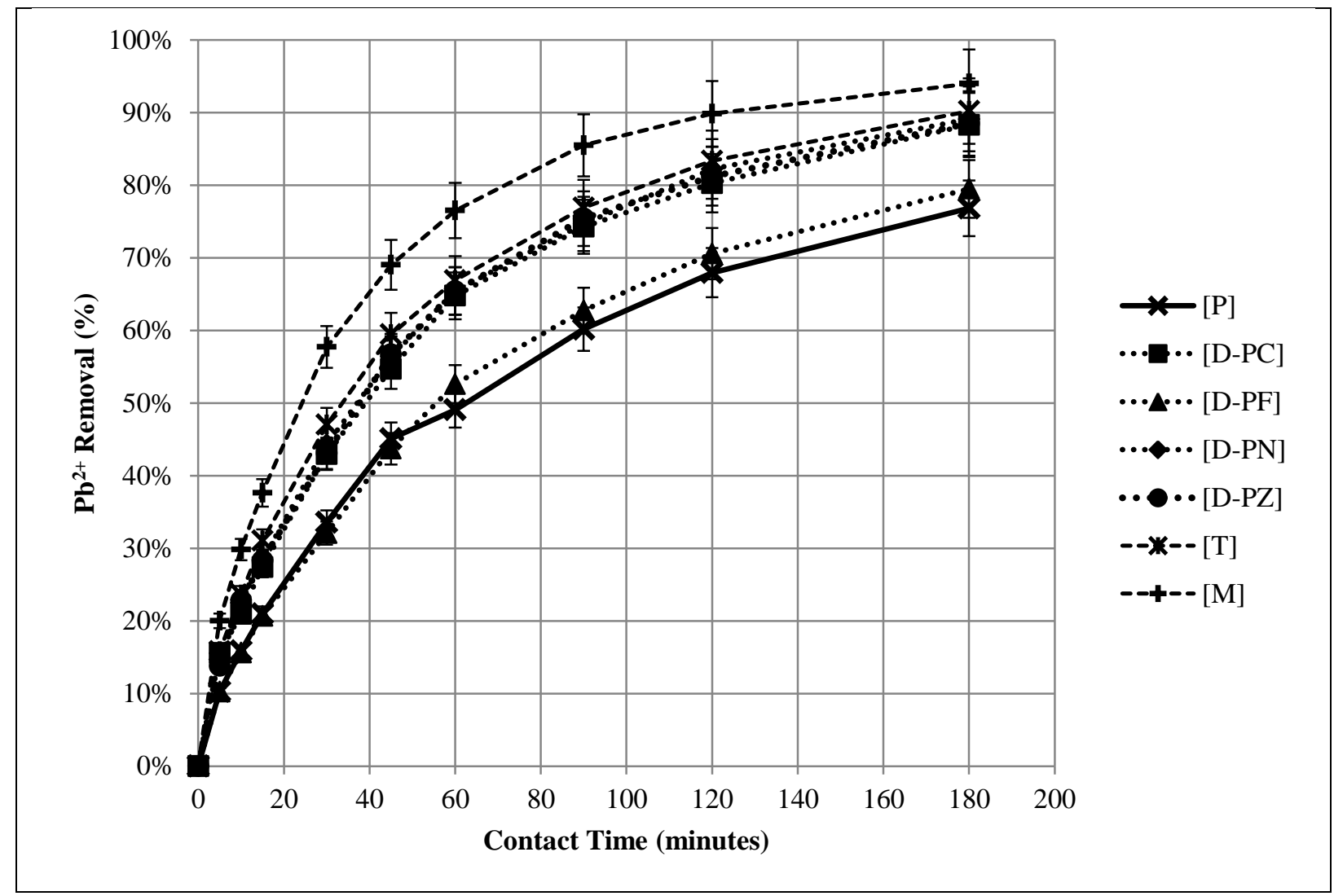

Figure 5.3 Percent Removal of Lead in the Various Component Systems 


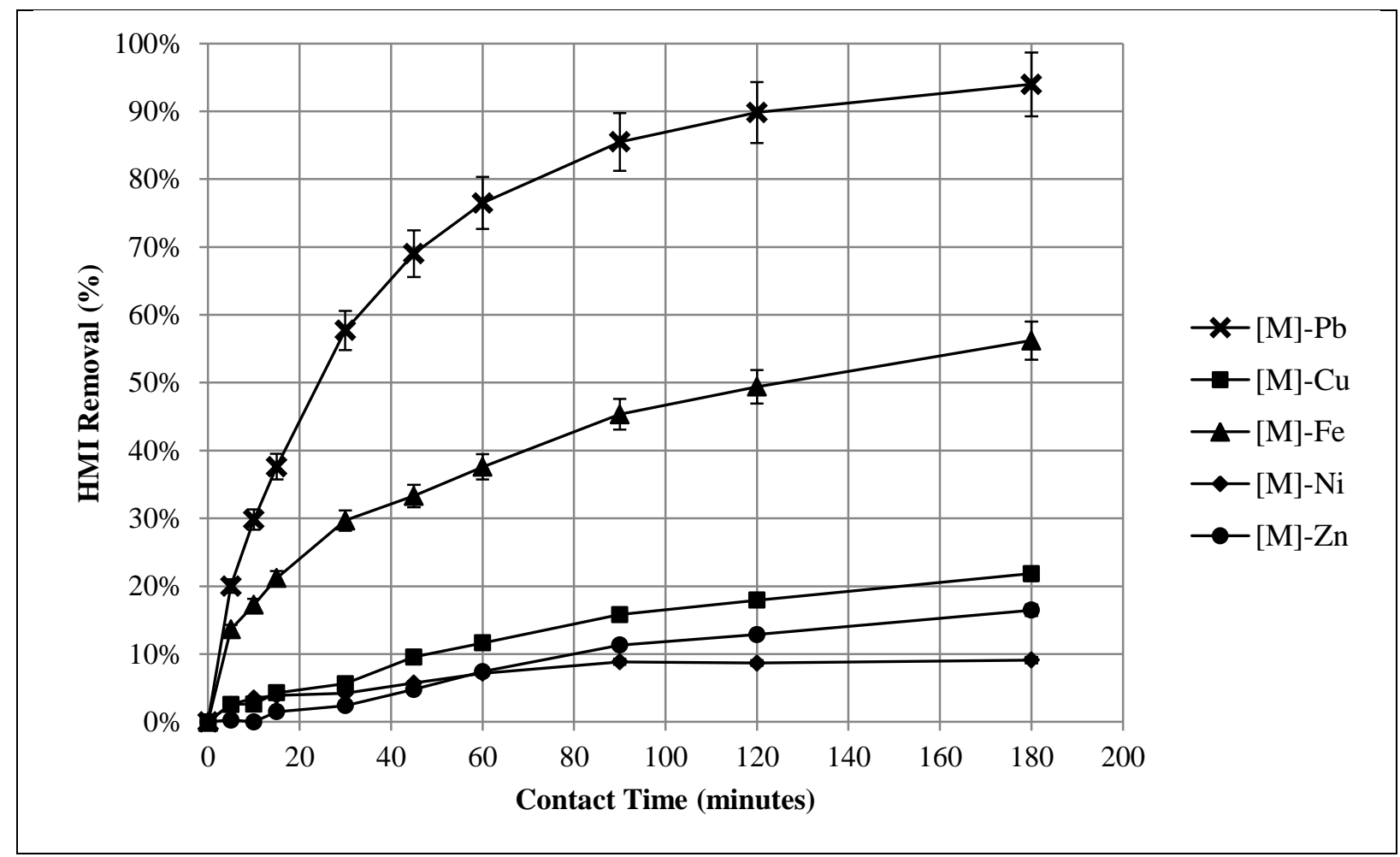

Figure 5.4 Percent Removal of HMIs in the Multi-Component System

Additional observations are revealed when the percent removal of each HMI of the various component systems is investigated further. With $\mathrm{Pb}^{2+}$ as the ion of interest in this study, Table 5.4 shows that only $1.88 \mathrm{meq} / \mathrm{L}$ out of a possible $2.0 \mathrm{meq} / \mathrm{L}$ in the multi-component system versus $7.68 \mathrm{meq} / \mathrm{L}$ of $10 \mathrm{meq} / \mathrm{L}$ in the single-component system. Zeolite's highest affinity is for lead, and is followed by iron. In the dual-iron [D-PF] system, $3.97 \mathrm{meq} / \mathrm{L}$ of $5.0 \mathrm{meq} / \mathrm{L}$ is removed and in the triple [T] system, $3.01 \mathrm{meq} / \mathrm{L}$ of $3.3 \mathrm{meq} / \mathrm{L}$ is removed once $\mathrm{Cu}^{2+}$ ion is introduced.

Table 5.4 The HMI Removal (meq/L) in the Various Component Systems Relative to the Lead Ion

\begin{tabular}{|c|c|c|c|c|c|c|c|c|}
\hline \multirow{2}{*}{\multicolumn{2}{|c|}{$\frac{\text { System }}{[\mathrm{P}]}$}} & & HMI & TOTAL & System & & HMI & TOTAL \\
\hline & & $\mathrm{Pb}^{2+}$ & \multicolumn{2}{|c|}{7.68} & \multirow{3}{*}{$\begin{array}{c}\text { TRIPLE } \\
{[\mathrm{T}]}\end{array}$} & $\mathrm{Pb}^{2+}$ & 3.01 & \multirow{3}{*}{5.29} \\
\hline \multirow{8}{*}{ DUAL } & \multirow{2}{*}{ [PC] } & $\mathrm{Pb}^{2+}$ & 4.41 & \multirow{2}{*}{5.03} & & $\mathrm{Cu}^{2+}$ & 0.63 & \\
\hline & & $\mathrm{Cu}^{2+}$ & 0.61 & & & $\mathrm{Fe}^{3+}$ & 1.65 & \\
\hline & \multirow{2}{*}[\mathrm{PF}]{} & $\mathrm{Pb}^{2+}$ & 3.97 & \multirow{2}{*}{5.86} & & & & \\
\hline & & $\mathrm{Fe}^{3+}$ & 1.89 & & \multirow{5}{*}{$\begin{array}{c}\text { MULTI } \\
{[\mathrm{M}]}\end{array}$} & $\mathrm{Pb}^{2+}$ & 1.88 & \multirow{5}{*}{3.95} \\
\hline & [DN] & $\mathrm{Pb}^{2+}$ & 4.46 & \multirow{2}{*}{4.82} & & $\mathrm{Cu}^{2+}$ & 0.44 & \\
\hline & {$[\mathrm{PN}]$} & $\mathrm{Ni}^{2+}$ & 0.37 & & & $\mathrm{Fe}^{3+}$ & 1.12 & \\
\hline & & $\mathrm{Pb}^{2+}$ & 4.42 & \multirow{2}{*}{4.97} & & $\mathrm{Ni}^{2+}$ & 0.18 & \\
\hline & {$[\mathrm{PZ}]$} & $\mathrm{Zn}^{2+}$ & 0.55 & & & $\mathrm{Zn}^{2+}$ & 0.33 & \\
\hline
\end{tabular}




\subsubsection{Selectivity Determination}

The removal efficiency order is indicative of how the adsorptive nature of zeolite for each ion varies among the different component systems. Based on the sorbent-to-sorbate ratio of $4 \mathrm{~g}$ to $100 \mathrm{~mL}$, the HMI uptake is calculated by applying Equation 3.2 and presented in Table 5.5. For a HMI pair evaluated as A/B, the selectivity ratio (SR) is determined by Equation $\mathbf{5 . 1}$ and presented in Table 5.6, where $\mathrm{q}_{\mathrm{t}}$ is the uptake at time $\mathrm{t}$ (in meq/g) (Inglezakis et al., 2003):

$$
\mathrm{SR}=\frac{\mathrm{q}_{\mathrm{t}, \mathrm{A}}}{\mathrm{q}_{\mathrm{t}, \mathrm{B}}}
$$

As HMIs are introduced into the system, the overall uptake of the lead ion and the total HMIs are decreased. An uptake of lead ions by zeolite is reduced by approximately $75.6 \%$, going from $0.1919 \mathrm{meq} / \mathrm{g}$ in the single-component [P] system to $0.0469 \mathrm{meq} / \mathrm{g}$ in the multi-component $[\mathrm{M}]$ system. Also, in the presence of highly competitive ions, the removal of $\mathrm{Pb}^{2+}$ is hindered. This is indicative of zeolite's selectivity, where the $\mathrm{Pb} / \mathrm{Fe}$ ratio is the lowest and the $\mathrm{Pb} / \mathrm{Ni}$ as the highest, with a greater uptake of lead in combination of $\mathrm{Ni}^{2+}$ than with $\mathrm{Fe}^{3+}$. Furthermore, $\mathrm{Cu}^{2+}$ is seen as the third ion of appeal to that of zeolite. When introduced in the triple-component system, it is observed that the overall uptake of HMIs is reduced from $0.1462 \mathrm{meq} / \mathrm{g}$ in the $[\mathrm{PF}]$ system to $0.1320 \mathrm{meq} / \mathrm{g}$ in the $[\mathrm{T}]$ system. These key trends are indicative of the selectivity of zeolite for the various HMIs in solution.

Table 5.5 The HMI Uptake in the Various Component Systems by Zeolite

\begin{tabular}{|c|c|c|c|c|c|c|c|c|}
\hline \multirow{2}{*}{\multicolumn{2}{|c|}{ System }} & \multirow{2}{*}{ HMIs } & \multicolumn{2}{|c|}{ Uptake (meq/g) } & \multirow{2}{*}{ System } & \multirow{2}{*}{ HMIs } & \multicolumn{2}{|c|}{ Uptake (meq/g) } \\
\hline & & & HMI & TOTAL & & & HMI & TOTAL \\
\hline \multicolumn{2}{|c|}{$[\mathrm{P}]$} & $\mathrm{Pb}^{2+}$ & \multicolumn{2}{|c|}{0.1919} & \multirow{2}{*}{$\begin{array}{c}\text { TRIPLE } \\
{[\mathrm{T}]}\end{array}$} & $\mathrm{Pb}^{2+}$ & 0.0750 & \multirow[b]{2}{*}{0.1320} \\
\hline \multirow{7}{*}{ DUAL } & [PC] & $\mathrm{Pb}^{2+}$ & 0.1102 & 0.1255 & & $\mathrm{Cu}^{2+}$ & 0.0158 & \\
\hline & & $\mathrm{Pb}^{2+}$ & 0.0992 & & & & & \\
\hline & {$[\mathrm{PF}]$} & $\mathrm{Fe}^{3+}$ & 0.0471 & 0.1462 & \multirow{5}{*}{$\begin{array}{c}\text { MULTI } \\
{[\mathrm{M}]}\end{array}$} & $\mathrm{Pb}^{2+}$ & 0.0469 & \multirow{5}{*}{0.0986} \\
\hline & & $\mathrm{Pb}^{2+}$ & 0.1112 & & & $\mathrm{Cu}^{2+}$ & 0.0109 & \\
\hline & {$[\mathrm{PN}]$} & $\mathrm{Ni}^{2+}$ & 0.0092 & 0.1204 & & $\mathrm{Fe}^{3+}$ & 0.0281 & \\
\hline & [P7] & $\mathrm{Pb}^{2+}$ & 0.1105 & \multirow{2}{*}{0.1243} & & $\mathrm{Ni}^{2+}$ & 0.0045 & \\
\hline & & $\mathrm{Zn}^{2+}$ & 0.0138 & & & $\mathrm{Zn}^{2+}$ & 0.0082 & \\
\hline
\end{tabular}

Table 5.6 Selectivity Ratios (SR) of the Various Component Systems Relative to Lead Uptake

\begin{tabular}{cccc}
\hline HMI Pair & $\begin{array}{c}\text { DUAL } \\
(5.0 \mathrm{meq} / \mathrm{L})\end{array}$ & $\begin{array}{c}\text { TRIPLE } \\
(3.3 \mathrm{meq} / \mathrm{L})\end{array}$ & $\begin{array}{c}\text { MULTI } \\
(2.0 \mathrm{meq} / \mathrm{L})\end{array}$ \\
\hline $\mathrm{Pb} / \mathrm{Cu}$ & 7.22 & 4.75 & 4.30 \\
$\mathrm{~Pb} / \mathrm{Fe}$ & 2.11 & 1.82 & 1.67 \\
$\mathrm{~Pb} / \mathrm{Ni}$ & 12.1 & --- & 10.3 \\
$\mathrm{~Pb} / \mathrm{Zn}$ & 8.04 & --- & 5.71 \\
\hline
\end{tabular}


Figure 5.5 represents the total uptake of HMI in the various component systems over the 3hour analysis period. It is evident that equilibrium has yet to be reached based on the established experimental conditions; however, a curve-to-plateau is detected and can be indicative of the long-term trends. As demonstrated by Motsi et al. (2009), the initial stage of rapid adsorption occurs within the first 40 minutes of contact. This is when all of the adsorption sites are available for cation interaction to occur, and when the concentration difference between the influent stock and sorbent-solution interface is very high. Inglezakis et al. (2002) credits this period to ionexchange in the micropores on the zeolite particles' surface. Slower sorption follows, which is attributed to slower diffusion of the cations in solution into the internal network of the zeolite, such that these cations must occupy the exchangeable sites within the channeled structure (Motsi et al., 2009).

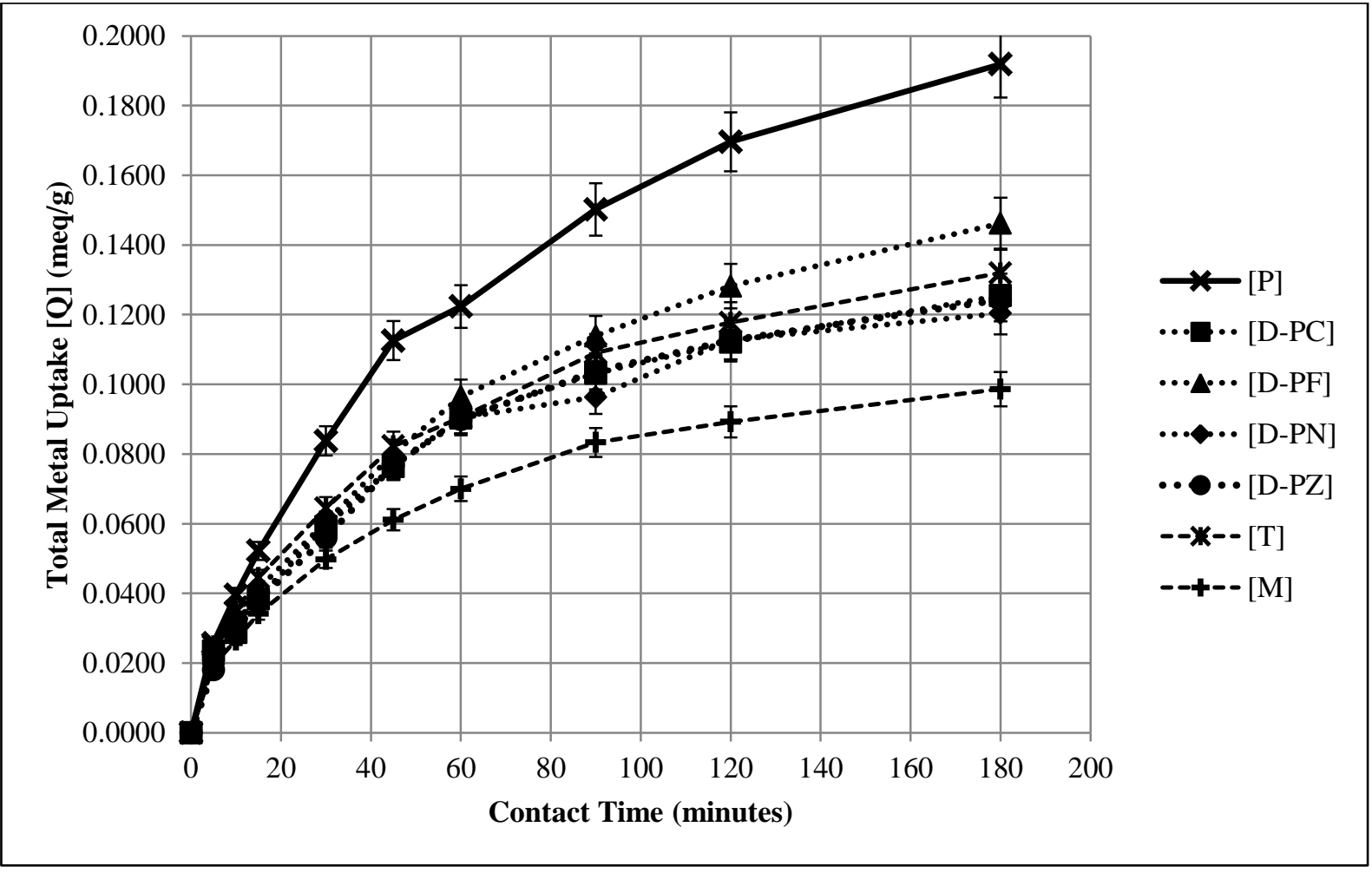

Figure 5.5 Total HMI Uptake in the Various Component Systems over Time

The presence of each HMI in solution impacts the uptake of lead; however, the removal efficiency trend is maintained throughout the various component systems. Once again, the sorbent mineral zeolite has a favoured uptake for that of the $\mathrm{Pb}^{2+}$ ion, followed by $\mathrm{Fe}^{3+}, \mathrm{Cu}^{2+}$, $\mathrm{Zn}^{2+}$, and then $\mathrm{Ni}^{2+}$. The selectivity study conducted by Anari-Anaraki and Nezamzadeh-Ejhieh 
(2015) involved surface surfactant modified clinoptilolite nanoparticles, with $\mathrm{Pb}^{2+}$ ion dualcomponent systems and interfering cations; supporting reported literature trends as: $\mathrm{Pb}^{2+}>\mathrm{Cd}^{2+}>$ $\mathrm{Cs}^{+}>\mathrm{Cu}^{2+}>\mathrm{Co}^{2+}>\mathrm{Cr}^{3+}>\mathrm{Zn}^{2+}>\mathrm{Ni}^{2+}$. This selectivity series is also consistent with the results obtained by Inglezakis et al. (2003), which investigated the trends of lead, copper, iron and chromium ions in various component systems as well as the findings of Ouki and Kavannagh (1997, 1999), who studied the selectivity and removal performance of lead, copper, cadmium, zinc, chromium, cobalt and nickel in a mixed system. Their research has determined that both physicochemical and stereochemical factors (i.e., micropore spacing constraints, hydrated radii (ion-to-pore ratio), cation hydration enthalpy) influence the removal efficiency.

Ouki and Kavannagh (1997) emphasize that clinoptilolite is comprised of a: (1) high Si/Al ratio, (2) low volumetric capacity, and (3) weak ionic field, within the structure. Therefore, the cation hydration free energy (a measure of water molecule rejection) (Inglezakis et al., 2003) is considered more significant than the electrostatic interactions within the framework during the sorption process. The HMIs with higher hydration energy prefer to remain in solution, where their hydration conditions are well-fulfilled. The hydration energy of $\mathrm{Pb}^{2+}(-357 \mathrm{kcal} / \mathrm{mol})$ compared to $\mathrm{Cu}^{2+}(-502 \mathrm{kcal} / \mathrm{mol})$ and $\mathrm{Fe}^{3+}(-1053 \mathrm{kcal} / \mathrm{mol})$ suggests that lead is superior in uptake to the other ions as it is not composed of complex, hydrated compounds and thereby demonstrates the significantly higher selectivity of lead by clinoptilolite (Ouki and Kavannagh, 1997; Inglezakis et al., 2003); this general observation is further substantiated by the contribution of this present study.

It is important to note that sorption equilibria is strongly affected by complexation of coions, counter-ions, and neutral molecules with one another; as a highly specific interaction, it may influence the selectivity of a given sorbent material (Helfferich, 1962, p. 202). Heavy metals are able to form stable complexes due to their electrostatic structure; $\mathrm{Cu}^{2+}, \mathrm{Fe}^{3+}$, and $\mathrm{Ni}^{2+}$ form stable complexes with water molecules, where these complexes are charged and give colour to their solutions (Inglezakis et al., 2004) (for the HMIs above, blue, orange/yellow, and green, respectively). As the metal concentration increases, metal ions which can no longer form outersphere complexes (e.g. ion-exchange) as a purely electrostatic attraction are forced into internal sites and start to form inner-sphere complexes (Xu et al., 2013) that are site-specific to the sorbent surface. This type of complexation is said to affect the reversibility of sorption reactions, 
given that inner-sphere complexation appears to be irreversible, due to the formation of covalent bonds (Xu et al., 2013). Specifically for the lead ion $\left(\mathrm{Pb}^{2+}\right)$ of interest to this study, the fact that the sorbent material prefers this counter-ion, which associates less strongly (least tendency to form strong complexes), compared to the co-ions in its framework is of importance. Consequently, the selectivity order observed in this study may be due to the fact that large and stable inorganic complexes may be mechanically excluded from the zeolitic structure by its unique sieve action properties. This may be the basis for the preferential uptake by the zeolite mineral for lead (Inglezakis et al., 2004).

In practical applications of industrial wastewater effluent, the presence of multiple HMIs in solution affects the overall treatment process. This study has demonstrated that the natural zeolite clinoptilolite is effective in the uptake of the target metal lead. Finally, further knowledge and quantitative details have been presented for using the sorption process as a valuable asset to wastewater treatment.

\subsection{Conclusions}

This study investigated the predominant heavy metallic ions found in mine waste streams, and how in combination, the removal specifically of lead $\left(\mathrm{Pb}^{2+}\right)$ is affected with their presence over time. The significant findings from this research are as follows:

1. Existing literature all agree that the $\mathrm{Pb}^{2+}$ ion can be removed by zeolite sorption; however, there are limitations on addressing the effect on this removal when other HMIs are present in the waste.

2. Qualitative observations by SEM/EDS imagery display that zeolite is influenced by the exposure to various heavy metallic ions in multi-component aqueous solutions.

3. While maintaining the $10 \mathrm{meq} / \mathrm{L}$ total $\mathrm{HMI}$ influent concentration, the removal of $\mathrm{Pb}^{2+}$ decreases from that in a single- to the multi-component system by approximately $75.6 \%$.

4. The systematic introduction of copper, iron, nickel and zinc to the system decreases the overall uptake of the total HMIs in solution. 
This removal method may be a predictive indicator for the removal of other metallic ions from other industrial effluents. The findings presented are significant both the mining and related environmental industry, which contribute to the advancement in the design of treatment systems through experimentation, modelling, and simulation.

\subsection{Future Works}

This study verifies that the zeolite mineral exhibits the greatest preference towards the lead $\left(\mathrm{Pb}^{2+}\right)$ ion with a selectivity series of $\mathrm{Pb}^{2+}>\mathrm{Fe}^{3+}>\mathrm{Cu}^{2+}>\mathrm{Zn}^{2+}>\mathrm{Ni}^{2+}$ and provides an understanding of how the HMIs affect the removal trends when combined. The next chapter addresses the gap in existing research that kinetic analysis is still very limited (Oter and Akcay, 2007; Motsi et al., 2011) in the number of HMIs of various multi-component system

combinations, with sorption by zeolite in its natural form (Panayotova and Velikov, 2002; Inglezakis et al., 2003; Bektas and Kara, 2004; Wang and Peng, 2010). 


\title{
6. KINETIC MODELLING TRENDS \\ Kinetic Modelling of the Removal of Multiple Heavy Metallic Ions \\ from Mine Waste by Natural Zeolite Sorption \\ Water-Special Issue \\ Treatment of Wastewater and Drinking Water through Advanced Technologies \\ 2017. 9(7). 482. doi:10.3390/w9070482.
}

\begin{abstract}
This study investigates the sorption of heavy metallic ions (HMIs), specifically lead $\left(\mathrm{Pb}^{2+}\right)$, copper $\left(\mathrm{Cu}^{2+}\right)$, iron $\left(\mathrm{Fe}^{3+}\right)$, nickel $\left(\mathrm{Ni}^{2+}\right)$ and zinc $\left(\mathrm{Zn}^{2+}\right)$, by natural zeolite (clinoptilolite). These HMIs are combined in single-, dual-, triple-, and multi-component systems. The batch-mode experiments consist of a total initial concentration of $10 \mathrm{meq} / \mathrm{L}$ normality for all systems, acidified to a $\mathrm{pH}$ of 2 by concentrated nitric $\left(\mathrm{HNO}_{3}\right)$ acid. A zeolite dosage of $4 \mathrm{~g}$ per $100 \mathrm{~mL}$ of synthetic nitrate salt aqueous solution is applied, for a contact period of 5 to $180 \mathrm{~min}$. Existing kinetic models on HMIs sorption are limited for multi-component system combinations. Therefore, this study conducts kinetic analysis by both reaction and diffusion models, to quantify the sorption process. The study concludes that the process correlates best with the pseudosecond-order (PSO) kinetic model. In the multi-component system combining all five HMIs, the initial sorption rate and theoretical equilibrium capacity are determined as $0.0033 \mathrm{meq} / \mathrm{g} \cdot \mathrm{min}$ and $0.1159 \mathrm{meq} / \mathrm{g}$, respectively. This provides significant insight into the mechanisms associated with the sorption process, as well as contributing to the assessment of natural zeolite as a sorbent material in its application in industrial wastewater treatment.
\end{abstract}




\subsection{Introduction}

The demand for proper evaluation and predication of water quality has grown, in order to protect surrounding water resources (Muttil and Chau, 2007; Wang et al., 2014). Environmental management is significant in any industry, which is a controlling factor for economic advancement, profitable development and the protection of the water resources at risk of future contamination (Wang et al., 2014). The environmental impact assessment (EIA) of large developments, such as industrial mining, is of great importance. The ecological, environmental, and socio-economic effects of these developments must be thoroughly evaluated. With this in mind, the EIA must establish both quantitative and qualitative standards with regards to modelling predication (Zhao et al., 2006).

In recent years, the study of sorption kinetics has attracted a lot of interest among researchers due to its importance in sorbent material assessment and subsequent application in the wastewater treatment industry (Qiu et al., 2009). Predicting the rate at which pollutant removal takes place in a given solid-solution system is crucial to establish an effective sorption system design (Plazinski et al., 2009). Many attempts have been made to generate an expression(s) to describe sorption kinetics on solid surfaces (i.e., natural zeolite, activated carbon, bio-sorbents, etc.) for solid-solution phase sorption systems (Ho and McKay, 1998a). Sorption kinetics defines the reaction pathways and uptake rates, along with residence times at which the equilibrium point is reached at the solid-solution interface (Beyazit, 2013). The physical as well as chemical composition of the sorbent material influences the sorption kinetics and controlling mechanisms (Kocaoba et al., 2007). A proper understanding of the mechanisms involved is not complete without experimental data on the simultaneous sorption of the heavy multi-metallic ions that are prevalent in industrial effluent, and an analysis on the uptake and rate of interference of these HMIs in combination (Helfferich, 1962; Borandegi and NezamzadehEjhieh, 2015). With the significant sorption research that has been undertaken, however, data is still very limited on kinetic analysis (Oter and Akcay, 2007; Motsi et al., 2011) of the number of HMIs in different multi-component system combinations, with sorption by zeolite in its natural form (Panayotova and Velikov, 2002; Inglezakis et al., 2003; Bektas and Kara, 2004; Wang and Peng, 2010). In light of this, the objective of the current study is to investigate the use of kinetic modelling to study the rate of the overall sorption process of the five (5) HMIs in this research 
project, by natural zeolite. To better understand how the removal of various ions may be affected by the presence of other HMIs, the experimental investigation will be carried out in increasing complexities, by combining the ions in single-, dual-, triple-, and multi-component aqueous solution systems. Various kinetic models, based on the reaction and diffusion concepts, will be considered in this study. It is envisaged that this kinetic analysis of the experimental data consisting of multiple HMIs in different combinations will provide significant insight into the mechanisms associated with the sorption process for industrial wastewater treatment (Qiu et al., 2009).

\subsection{Materials and Methods}

Refer to Chapter 3 for the consolidated experimental methodology; sorbent material, sorbate solution, and analytical procedure. All analyses are conducted in batch-mode, creating the synthetic simple solute HMI solution in the various component system combinations at a 4-g sorbent mass to $100-\mathrm{mL}$ sorbate volume dosage. The following subsections discuss the associated principles of sorption kinetics and the model selection rationale for this study.

\subsubsection{Sorption Kinetics Principles}

The accuracy of modelling prediction is dependent on the various open boundary conditions, model parameters selected, and numerical method(s) implemented. The variation in sorption kinetics is attributed to the specific crystalline structure (physical as well as chemical composition) of the sorbent material, and is controlled by various mechanisms (Helfferich, 1962; Bekkum et al., 1991; Kocaoba et al., 2007; Motsi et al., 2011). Kinetic modelling is a powerful tool to assess the performance of sorbent materials and to comprehend these fundamental mechanisms involved in the sorption process. This is classified as follows (Qiu et al., 2009; Motsi et al., 2011), where either one sole-step or multiple phases have a predominate impact (Fierro et al., 2008): 
1. Reaction-Type Model

Chemisorption - chemical reaction at the sites of the functional ionic groups within the zeolite and focuses on the process as a whole.

2. Diffusion-Type Model

a. Film Diffusion - diffusion of counter-ions in the adherent layer surrounding the zeolite (between the external solution and crystal surface).

b. Intra-Particle Diffusion - diffusion of counter-ions in the solute within the zeolite pores and/or along the pore walls.

c. Mass Action - sorption or desorption between the active sites and the sorbate material.

Both reaction-type and diffusion-type sorption kinetic classifications have been investigated; correlations between the sorption uptake of the HMIs with respect to time were analyzed specifically in this study. The most prevalent models (Ho and McKay, 1998a; Bektas and Kara, 2004; Sprynskyy et al., 2006; Fierro et al., 2008; Qiu et al., 2009; Palzinski et al., 2009; Motsi et al., 2011; Jovanovic et al., 2012; Olu-Owolabi et al., 2014; Farouq and Yousef, 2015; Largitte and Pasquier, 2016) considered in the model selection process are discussed in the following subsections.

\subsubsection{Reaction-Type Models}

\section{Pseudo-First-Order (PFO) Rate Equation}

This model is considered to be earliest to represent the liquid-solid phase sorption process, relating the capacity to rate (Qiu et al., 2009). The general relationship is given in Equation 6.1. By applying the boundary conditions of $t=0 \rightarrow q_{t}=0$ and $t=t \rightarrow q_{t}=q_{t}$, its solution and linearized form are given in Equation 6.2 and Equation 6.3, respectively (Ho and McKay, 1998a; Bektas and Kara, 2004; Qiu et al., 2009; Farouq and Yousef, 2015):

$$
\begin{gathered}
\frac{d q_{t}}{d t}=k_{1}\left(q_{e}-q_{t}\right) \\
k_{1} t=\ln \left(\frac{q_{e}}{q_{e}-q_{t}}\right) \\
\log \left(q_{e}-q_{t}\right)=\log q_{e}-\left(\frac{k_{1}}{2.303}\right) t
\end{gathered}
$$


where $\mathrm{q}_{\mathrm{e}}$ and $\mathrm{q}_{\mathrm{t}}$ are sorption capacity (the amount of HMI sorbed per unit weight of sorbent) (in meq/g) at equilibrium and at time $\mathrm{t}$ (in min), respectively; and $\mathrm{k}_{1}$ is the pseudo-first order rate constant (in $\left.\min ^{-1}\right)$. According to this model, a plot of $\log \left(\mathrm{q}_{\mathrm{e}}-\mathrm{q}_{\mathrm{t}}\right)$ versus $\mathrm{t}$ would provide a linear trend (Fierro et al., 2008).

\section{Pseudo-Second-Order (PSO) Rate Equation}

This model implies that the rate-determining (RDS) step is by chemical adsorption (chemisorption), involving valent forces through sharing or electron exchange between the sorbent material and the HMIs in solution. The sorption rate depends on the amount of ions on the sorbent surface at time $t$ and what is sorbed at equilibrium (Qiu et al., 2009). The general relationship is given in Equation 6.4. By applying the boundary conditions of $t=0 \rightarrow q_{t}=0$ and $\mathrm{t}=\mathrm{t} \rightarrow \mathrm{q}_{\mathrm{t}}=\mathrm{q}_{\mathrm{t}}$, its linearized form is given in Equation 6.5 and rearranged in Equation 6.6 (Ho and McKay, 1998a; Bektas and Kara, 2004; Sprynskyy et al., 2006; Qiu et al., 2009; Motsi et al., 2011; Farouq and Yousef, 2015):

$$
\begin{aligned}
\frac{\mathrm{dq}}{\mathrm{dt}} & =\mathrm{k}_{2}\left(\mathrm{q}_{\mathrm{e}}-\mathrm{q}_{\mathrm{t}}\right)^{2} \\
\frac{\mathrm{t}}{\mathrm{q}_{\mathrm{t}}} & =\frac{\mathrm{t}}{\mathrm{q}_{\mathrm{e}}}+\frac{1}{\mathrm{k}_{2} \mathrm{q}_{\mathrm{e}}^{2}} \\
\mathrm{q}_{\mathrm{t}} & =\frac{\mathrm{k}_{2} \mathrm{q}_{\mathrm{e}}^{2} \mathrm{t}}{\left[1+\mathrm{k}_{2} \mathrm{q}_{\mathrm{e}} \mathrm{t}\right]}
\end{aligned}
$$

where $\mathrm{h}=\mathrm{k}_{2} \mathrm{q}_{\mathrm{e}}^{2}$ is the initial sorption rate (in $\mathrm{meq} / \mathrm{g} \cdot \mathrm{min}$ ) as $\mathrm{t}$ approaches zero (Ho et al., 2006), and $\mathrm{k}_{2}$ is the PSO rate constant (in $\mathrm{g} / \mathrm{meq} \cdot \mathrm{min}$ ). These constants are determined by a plot of the linearized form (Equation 6.5) of $\mathrm{t} / \mathrm{q}_{\mathrm{t}}$ versus $\mathrm{t}$ (Bektas and Kara, 2004; Ho et al., 2006; Kocaoba et al., 2007; Qiu et al., 2009).

\section{The Elovich Equation}

This model was developed to predominately describe the sorption interactions between the gas phase onto a heterogeneous solid surfaces (Plazinski et al.; 2009; Qiu et al., 2009; Farouq and Yousef, 2015), but has also represented sorption of contaminants from aqueous solutions (Fierro et al., 2008). The general relationship is given in Equation 6.7. By applying the boundary conditions of $\mathrm{t}=0 \rightarrow \mathrm{q}_{\mathrm{t}}=0$ and $\mathrm{t}=\mathrm{t} \rightarrow \mathrm{q}_{\mathrm{t}}=\mathrm{q}_{\mathrm{t}}$, and the assumption that $\alpha \beta \mathrm{t} \gg 1$, 
its linearized form is given in Equation 6.8. (Ho and McKay, 1998a; Fierro et al., 2008; Qiu et al., 2009; Olu-Owolabi et al., 2014; Farouq and Yousef, 2015; Largitte and Pasquier, 2016):

$$
\begin{gathered}
\frac{\mathrm{dq}_{\mathrm{t}}}{\mathrm{dt}}=\alpha \cdot \exp \left(-\beta \mathrm{dq}_{\mathrm{t}}\right) \\
\mathrm{q}_{\mathrm{t}}=\left(\frac{1}{\beta}\right)[\ln (\mathrm{t})]+\left(\frac{1}{\beta}\right)[\ln (\alpha \beta)]
\end{gathered}
$$

where $\mathrm{q}_{\mathrm{t}}$ is the amount sorbed at time $\mathrm{t}, \beta$ is the Elovich constant (in $\mathrm{g} / \mathrm{meq}$ ) (Wu et al., 2009) (relating to the extent of surface coverage (site availability) (Fierro et al., 2008) and activation energy for chemisorptions) (Farouq and Yousef, 2015), and $\alpha$ is the initial sorption rate (in meq/g.min) (Ho and McKay, 1998a; Wu et al., 2009). According to this model, a plot of $\mathrm{q}_{\mathrm{t}}$ versus $\ln (\mathrm{t})$ would provide a linear trend (Ho and McKay, 1998a; Fierro et al., 2008).

\subsubsection{Diffusion-Type Models}

Sorption kinetics may be described from a systematic point of view. Of the three steps involved in the diffusion kinetic category described, mass action is very rapid, and therefore considered negligible in kinetic analysis (Qiu et al., 2009). The Weber-Morris intra-particle diffusion (IPD) model and its parameters provide significant information in order to determine whether film diffusion or intra-particle diffusion is the RDS. The IPD model demonstrates that in numerous sorption studies, the ion solute uptake varies almost proportionately with $\sqrt{t}$ rather than with time $t$ (Bektas and Kara, 2004; Kocaoba et al., 2007; Qiu et al., 2009). If diffusion is the sorption mechanism, then the plot of $q_{t}$ versus $\sqrt{t}$, as expressed in Equation 6.9, is linear (Bektas and Kara, 2004; Kocaoba et al., 2007; Fierro et al., 2008; Qiu et al., 2009; Zolgharnein and Shahmoradi, 2010; Olu-Owolabi et al., 2014):

$$
\mathrm{q}_{\mathrm{t}}=\mathrm{k}_{\mathrm{P}} \cdot(\sqrt{\mathrm{t}})+\mathrm{C}
$$

where $\mathrm{k}_{\mathrm{P}}$ (in meq/g. $\min ^{1 / 2}$ ) is the intra-particle diffusion rate constant, and $\mathrm{C}$ (in meq/g) is a constant representing the thickness of the boundary layer (Fierro et al., 2008; Olu-Owolabi et al., 2014); the greater the $C$ value, the greater the boundary layer effect. Further, the intra-particle diffusion mechanism specifically is the RDS when this plot intersects the origin (Fierro et al., 2008; Qiu et al., 2009). When the sorption process is controlled by more than one diffusion mechanism, then the data plot deviates from the origin with a multi-linear trend; the mechanism 
of sorption is complex and both external and intra-particle diffusion play a role in the actual sorption process (Zolgharnein and Shahmoradi, 2010). The initial steep phase implies that the external resistance to mass transfer surrounding the particles is significant, representing macropore and mesopore diffusion. The second gradual phase implies micropore intra-particle diffusion as the RDS (Fierro et al., 2008).

\subsubsection{Kinetic Model Selection}

The mechanism study conducted by Sprynskyy et al. (2006) demonstrates that the sorption of HMIs $\left(\mathrm{Pb}^{2+}, \mathrm{Cu}^{2+}, \mathrm{Ni}^{2+}, \mathrm{Cd}^{2+}\right)$ by natural zeolite is a heterogeneous process with three distinct stages. First, a very fast (instantaneous) uptake occurs within the first 30 minutes, observed for four HMIs at three different concentrations. This is followed by inversion due to desorption prevalence, observed predominantly for $\mathrm{Ni}^{2+}$ at all concentrations and overall increases for all four HMIs at higher metal concentrations. Finally, a slower uptake increase of all four HMIs is observed, with a majority of $\mathrm{Ni}^{2+}$ occurring in the first stage. In the kinetic studies of HMI uptake $\left(\mathrm{Fe}^{3+}, \mathrm{Cu}^{2+}, \mathrm{Mn}^{2+}, \mathrm{Zn}^{2+}\right)$ by natural zeolite conducted by Motsi et al. (2009), the initial stage of rapid adsorption occurs within the first 40 minutes of contact. This is when all of the adsorption sites are available for cation interaction to occur, and when the concentration difference between the influent stock and sorbent-sorbate interface is very high. Inglezakis et al. (2002) credits this period to ion-exchange in the micropores on the zeolite particles' surface. During the second stage, desorption prevalence is most likely caused by slower diffusion of exchangeable co-ions within the internal network $\left(\mathrm{Ca}^{2+}, \mathrm{Na}^{+}, \mathrm{K}^{+}\right)$of the zeolite crystalline framework, and consequently occupies the exchange positions on the surface to the counter-ions (HMIs) in solution. During the third stage, a gradual sorption in the micropores within the crystalline occurs. Deceleration in the close micropores within the framework is connected by poor access as well as by more intensive sorption in comparison with in the surface micropores. 
Under the testing conditions of this study, the sorption capacity at equilibrium $\left(\mathrm{q}_{\mathrm{e}}\right)$ was not experimentally determined (due to the 3-hour contact time limitation); which must be known in order to fit the data to the PFO model equation. In addition, previous research efforts have demonstrated that the equation does not fit the data as well for the complete contact time range (Bektas and Kara, 2004; Fierro et al., 2008; Zolgharnein and Shahmoradi, 2010; Olu-Owolabi et al., 2014). The Elovich equation can be associated with the model of a strongly heterogeneous solid surface. However, recent studies show that the PSO and Elovich equations exhibit very similar behaviour under the assumption that the system is not close to equilibrium (Plazinski et al., 2009). The PSO model assumes: (1) sorption only occurs on localized sites and involves no interaction between the sorbed ions; (2) the energy of adsorption is not dependent on surface coverage; (3) maximum adsorption corresponds to a saturated mono-layer of adsorbates on the adsorbent surface, (4) the concentration of the sorbate is considered to be constant, and (5) the metal ion uptake on the activated carbons is governed by a second-order rate equation. The Elovich model differs in the first two assumptions in that: (1) sorption only occurs on localized sites and there is inter-action between the sorbed ions; and (2) the energy of adsorption increases linearly with the surface coverage (Largitte and Pasquier, 2016).

The PSO model has effectively demonstrated the sorption process of various contaminants, including metal ions, and organic substances in an aqueous state (Fierro et al., 2008; Qiu et al., 2009; Zolgharnein and Shahmoradi, 2010; Jovanovic et al., 2012; Olu-Owolabi et al., 2014). Several literature review findings of kinetic models of the removal specifically of heavy metals from aqueous solutions by natural clinoptilolite were considered by Jovanovic et al. (2012), which widely described by researchers that the sorption process by the PSO model is the best representation. However, the system specific presumptions of system variables (Ho and McKay, 1998a) (operation conditions) (i.e., sorbent particle size, counter-ions' initial concentration, temperature, $\mathrm{pH}$ level, degree of agitation, etc.) must be stated, which affect the mechanisms involved. The experimental data that fits to the PSO model indicates that chemisorption (chemical reaction) is the rate-determining step (RDS), which is the case if the rate coefficient is constant for each operative condition. If any of these conditions are not satisfied, chemisorption is not the RDS even if the data fits the PSO kinetic model well (Motsi et al., 2011). Motsi et al. (2011) determined that intra-particle diffusion is the primary RDS in the uptake of HMIs $\left(\mathrm{Fe}^{3+}\right.$, 
$\left.\mathrm{Cu}^{2+}, \mathrm{Mn}^{2+}, \mathrm{Zn}^{2+}\right)$ by natural zeolite and suggested that electric transference during intra-particle diffusion is a significant event in the sorption process.

Based on the above discussion and preliminary research findings, the study presented in this paper will be carried out with these two selected models: (1) the PSO reaction model and (2) the IPD model. The objective is to observe the various uptake rates and mechanisms associated of the two models, thereby resulting in an assessment and comparison of the final performance of the two kinetic models developed from distinctly different premises.

\subsection{Quality Control (QC) Protocol}

For all analytical sessions, the triplicate concentration of the median standard $(50 \mathrm{mg} / \mathrm{L})$ detected an average of $51.62 \mathrm{mg} / \mathrm{L}$, and is within $5 \%$ of the known value; the percent relative standard deviation (\%-RSD) reported an average of $0.597 \%$, which is well within the $\leq 3 \%$ limit; the correlation coefficient of each HMI analyte primary wavelength generated an average of 0.9998 , which is very close to unity.

\subsection{Analysis}

\subsubsection{Acidity Levels}

Following sorbate-sorbent contact, the $\mathrm{pH}$ is observed to be steady throughout the sorption process. The maximum recorded $\mathrm{pH}$ values are 2.16, 2.21, 2.19, and, 2.17 for the single-, dual-, triple-, and multi-component systems, respectively; for both at day of zeolite exposure and the day after of filtered sample. The $\mathrm{pH}$ levels of the prepared influent stock and filtered effluent are also observed to be constant during refrigerated storage prior to dilution preparation for elemental analysis. This present study was conducted in the conservative manner, with all $\mathrm{pH}$ values documented to be below reported ranges and within comparability. By maintaining very low $\mathrm{pH}$ levels in the batch experiments and the use of highly soluble nitrate salts, the precipitation of the HMIs is avoided. Additional trials verified that the filtered and unfiltered HMI influent stock concentrations are the same, indicating both effective dilution practices and 
complete solubility. Therefore, the concentration of each HMI before and after batch sorption was exclusively in soluble form.

\subsubsection{Heavy Metallic Ion Levels}

The ICP-AES results of the various component systems at 3 contact hours are summarized in Table 6.1. to Table 6.3. The primary wavelengths 'Analyte' are given in Column 1, and the 'Sample ID' in Column 2 provides the systems' influent stock (S) and 180 contact minute data. In order to keep all concentrations within the calibration range of $0-100 \mathrm{mg} / \mathrm{L}$, the samples are diluted by $50 \%$ with deionized distilled water, indicated by the superscript value. As observed in the quality control measures for the calibration standard, the \%-RSD values are low and all within $<3 \%$ as given in Column 3 , which is indicative that the data is accurate and the experimental replicates are reliable (Perkin Elmer Inc., 2010). Triplicate readings and their mean concentrations in calibration units in $\mathrm{mg} / \mathrm{L}$ are presented in Column 4. The dilution factors in Column 2 are applied to the triplicate concentrations, and the corresponding HMI concentrations are given in Column 5. By applying Equation 3.2, the uptake sorption capacity is given in Column 6. The percent removal after 180 contact minutes is calculated in Column 7. Before kinetic analysis is conducted, the overall removal efficiency order indicates that the $\mathrm{Pb}^{2+}$ ion is highest compared to the four other HMIs investigated. This order is consistently high regardless of the component system combinations (Panayotova and Velikov, 2002; Oter and Akcay, 2007).

Figure 6.1 presents the percent removal of the total HMIs found in the single-lead [P], triple-(lead-copper-iron) [T], and multi-[M] component systems over the 3 hour analysis period. The HMIs are progressively added to the aqueous solution while maintaining the total HMI concentration of $10 \mathrm{meq} / \mathrm{L}$. The overall uptake is reduced from [P] to [T] by $0.0599 \mathrm{meq} / \mathrm{g}$ or $31.2 \%$, and from [T] to [M] by $0.0334 \mathrm{meq} / \mathrm{g}$ or $25.3 \%$. It is visually evident that each HMI in solution distinctively impacts the overall uptake. An equilibrium state has yet to be reached based on the established experimental conditions. Yet, the curve-to-plateau trend shows three distinct stages, where rapid uptake is observed within the first 45 contact minutes; as demonstrated by Sprynskyy et al. (2006) and Motsi et al. (2009). This is a first glimpse in this study of zeolite's preference for the HMIs to be investigated, indicative of the long-term trends. 
Table 6.1 ICP-AES HMI Results of the Single-Component Systems

\begin{tabular}{|c|c|c|c|c|c|c|}
\hline 1 & 2 & 3 & 4 & 5 & 6 & 7 \\
\hline \multirow{2}{*}{ Analyte } & \multirow{2}{*}{ Sample ID } & \multirow{2}{*}{$\%$-RSD } & \multicolumn{2}{|c|}{ Concentration (mg/L) } & \multirow{2}{*}{$\mathrm{meq} / \mathrm{g}$} & \multirow{2}{*}{$\% \mathbf{R}$} \\
\hline & & & Triplicate Mean (Calib) & DIL & & \\
\hline \multirow{2}{*}{$\mathrm{Pb} 220.353$} & {$[\mathrm{P}]-\mathrm{S}^{4}$} & 0.24 & 75.93 & 1214.81 & \multirow{2}{*}{0.1919} & \multirow{2}{*}{76.8} \\
\hline & {$[\mathrm{P}] 180^{3}$} & 0.59 & 35.20 & 281.63 & & \\
\hline \multirow{2}{*}{$\mathrm{Cu} 327.393$} & {$[\mathrm{C}]-\mathrm{S}^{2}$} & 0.40 & 105.37 & 421.49 & \multirow{2}{*}{0.0533} & \multirow{2}{*}{21.4} \\
\hline & {$[\mathrm{C}] 180^{2}$} & 0.16 & 82.84 & 331.37 & & \\
\hline \multirow[b]{2}{*}{ Fe 238.204 } & {$[\mathrm{F}]-\mathrm{S}^{1}$} & 0.53 & 105.83 & 211.65 & \multirow[b]{2}{*}{0.0757} & \multirow[b]{2}{*}{30.3} \\
\hline & {$[\mathrm{F}] 180^{1}$} & 0.55 & 73.73 & 147.46 & & \\
\hline \multirow{2}{*}{ Ni 231.604} & {$[\mathrm{~N}]-\mathrm{S}^{2}$} & 0.32 & 87.30 & 349.19 & \multirow{2}{*}{0.0268} & \multirow{2}{*}{10.7} \\
\hline & {$[\mathrm{N}] 180^{2}$} & 0.36 & 77.92 & 311.69 & & \\
\hline \multirow{2}{*}{ Zn 206.200} & {$[\mathrm{Z}]-\mathrm{S}^{2}$} & 1.10 & 104.67 & 418.68 & \multirow{2}{*}{0.0494} & \multirow{2}{*}{19.8} \\
\hline & {$[\mathrm{Z}] 180^{2}$} & 0.74 & 83.94 & 335.77 & & \\
\hline
\end{tabular}

Table 6.2 ICP-AES HMI Results of the Dual-Component Systems

\begin{tabular}{|c|c|c|c|c|c|c|}
\hline 1 & 2 & 3 & 4 & 5 & 6 & 7 \\
\hline \multirow{2}{*}{ Analyte } & \multirow{2}{*}{ Sample ID } & \multirow{2}{*}{$\%$-RSD } & \multicolumn{2}{|c|}{ Concentration $(\mathrm{mg} / \mathrm{L})$} & \multirow{2}{*}{$\mathrm{meq} / \mathrm{g}$} & \multirow{2}{*}{$\% \mathbf{R}$} \\
\hline & & & Triplicate Mean (Calib) & DIL & & \\
\hline \multirow{2}{*}{$\mathrm{Pb} 220.353$} & {$[\mathrm{D}-\mathrm{PC}]-\mathrm{S}^{3}$} & 0.13 & 75.47 & 603.79 & \multirow{2}{*}{0.1102} & \multirow{2}{*}{88.3} \\
\hline & {$[\mathrm{D}-\mathrm{PC}] 180^{2}$} & 0.59 & 17.68 & 70.73 & & \\
\hline \multirow{2}{*}{$\mathrm{Cu} 327.393$} & {$[\mathrm{D}-\mathrm{PC}]-\mathrm{S}^{3}$} & 0.11 & 24.18 & 193.44 & \multirow{2}{*}{0.0153} & \multirow{2}{*}{12.2} \\
\hline & {$[\mathrm{D}-\mathrm{PC}] 180^{2}$} & 0.61 & 42.44 & 169.77 & & \\
\hline \multirow{2}{*}{$\mathrm{Pb} 220.353$} & {$[\mathrm{D}-\mathrm{PF}]-\mathrm{S}^{3}$} & 0.42 & 75.98 & 607.82 & \multirow{2}{*}{0.0992} & \multirow{2}{*}{79.5} \\
\hline & {$[\mathrm{D}-\mathrm{PF}] 180^{2}$} & 0.54 & 31.19 & 124.77 & & \\
\hline \multirow{2}{*}{ Fe 238.204 } & {$[\mathrm{D}-\mathrm{PF}]-\mathrm{S}^{3}$} & 0.31 & 13.01 & 104.11 & \multirow{2}{*}{0.0471} & \multirow{2}{*}{37.7} \\
\hline & {$[\mathrm{D}-\mathrm{PF}] 180^{2}$} & 0.45 & 16.21 & 64.82 & & \\
\hline \multirow{2}{*}{$\mathrm{Pb} 220.353$} & {$[\mathrm{D}-\mathrm{PN}]-\mathrm{S}^{3}$} & 0.82 & 77.16 & 617.29 & \multirow{2}{*}{0.1112} & \multirow{2}{*}{89.1} \\
\hline & {$[\mathrm{D}-\mathrm{PN}] 180^{2}$} & 0.68 & 16.81 & 67.22 & & \\
\hline \multirow{2}{*}{ Ni 231.604} & {$[\mathrm{D}-\mathrm{PN}]-\mathrm{S}^{3}$} & 0.86 & 21.05 & 168.37 & \multirow{2}{*}{0.0092} & \multirow{2}{*}{7.40} \\
\hline & {$[\mathrm{D}-\mathrm{PN}] 180^{2}$} & 0.12 & 38.99 & 155.94 & & \\
\hline \multirow{2}{*}{$\mathrm{Pb} 220.353$} & {$[\mathrm{D}-\mathrm{PZ}]-\mathrm{S}^{3}$} & 1.01 & 76.78 & 614.22 & \multirow{2}{*}{0.1105} & \multirow{2}{*}{88.5} \\
\hline & {$[\mathrm{D}-\mathrm{PZ}] 180^{2}$} & 0.50 & 17.68 & 70.72 & & \\
\hline \multirow{2}{*}{ Zn 206.200} & {$[\mathrm{D}-\mathrm{PZ}]-\mathrm{S}^{3}$} & 0.92 & 23.46 & 187.65 & 0.0138 & 110 \\
\hline & {$[\mathrm{D}-\mathrm{PZ}] 180^{2}$} & 0.83 & 41.75 & 166.98 & 0.0150 & 11.0 \\
\hline $\mathrm{Cu} 327.393$ & {$[\mathrm{D}-\mathrm{CF}]-\mathrm{S}^{1}$} & 0.90 & 95.76 & 191.51 & 00283 & 227 \\
\hline cuszl.j95 & {$[\mathrm{D}-\mathrm{CF}] 180^{1}$} & 0.58 & 74.06 & 148.13 & 0.0203 & 22.1 \\
\hline & {$[\mathrm{D}-\mathrm{CF}]-\mathrm{S}^{1}$} & 2.75 & 50.61 & 101.21 & 00534 & \\
\hline Fe 258.204 & {$[\mathrm{D}-\mathrm{CF}] 180^{1}$} & 0.70 & 28.95 & 57.90 & 0.0534 & 42.8 \\
\hline Си 327393 & {$[\mathrm{D}-\mathrm{CN}]-\mathrm{S}^{1}$} & 0.50 & 94.92 & 189.85 & 00361 & 28.9 \\
\hline cus 321.593 & {$[\mathrm{D}-\mathrm{CN}] 180^{1}$} & 0.40 & 67.46 & 134.92 & 0.0361 & 20.9 \\
\hline $\mathrm{Ni} 231604$ & {$[\mathrm{D}-\mathrm{CN}]-\mathrm{S}^{1}$} & 0.23 & 80.53 & 161.06 & 00125 & 100 \\
\hline 101251.004 & {$[\mathrm{D}-\mathrm{CN}] 180^{1}$} & 0.48 & 72.48 & 144.96 & $0.012 \mathrm{~J}$ & 10.0 \\
\hline & {$[\mathrm{D}-\mathrm{FZ}]-\mathrm{S}^{1}$} & 2.31 & 51.15 & 102.30 & & \\
\hline Fe 238.204 & {$[\mathrm{D}-\mathrm{FZ}] 180^{1}$} & 0.20 & 28.78 & 57.56 & 0.0546 & 43.1 \\
\hline & {$[\mathrm{D}-\mathrm{FZ}]-\mathrm{S}^{1}$} & 2.69 & 90.55 & 181.09 & & \\
\hline Zn 206.200 & {$[\mathrm{D}-\mathrm{FZ}] 180^{1}$} & 0.22 & 73.43 & 146.85 & 0.0236 & 18.9 \\
\hline & {$[\mathrm{D}-\mathrm{NZ}]-\mathrm{S}^{1}$} & 1.22 & 80.15 & 160.31 & & \\
\hline $\mathrm{N} 1231.604$ & {$[\mathrm{D}-\mathrm{NZ}] 180^{1}$} & 3.49 & 75.85 & 151.71 & $0.006 /$ & 5.4 \\
\hline $7 n 206.200$ & {$[\mathrm{D}-\mathrm{NZ}]-\mathrm{S}^{1}$} & 1.30 & 89.51 & 179.02 & 00244 & 196 \\
\hline 20200.200 & {$[\mathrm{D}-\mathrm{NZ}] 180^{1}$} & 3.84 & 71.99 & 143.99 & 0.0244 & 19.0 \\
\hline
\end{tabular}


Table 6.3 ICP-AES HMI Results of the Triple- and Multi-Component Systems

\begin{tabular}{|c|c|c|c|c|c|c|}
\hline 1 & 2 & 3 & 4 & 5 & 6 & 7 \\
\hline \multirow[b]{2}{*}{ Analyte } & \multirow[b]{2}{*}{ Sample ID } & \multirow[b]{2}{*}{$\%$-RSD } & \multicolumn{2}{|c|}{ Concentration (mg/L) } & \multirow[b]{2}{*}{ meq/g } & \multirow[b]{2}{*}{$\% \mathbf{R}$} \\
\hline & & & $\begin{array}{c}\text { Triplicate } \\
\text { Mean (Calib) }\end{array}$ & DIL & & \\
\hline \multirow{2}{*}{$\begin{array}{c}\mathrm{Pb} \\
220.353\end{array}$} & {$[\mathrm{~T}]-\mathrm{S}^{2}$} & 1.27 & 100.05 & 400.21 & \multirow{2}{*}{0.0750} & \multirow{2}{*}{90.2} \\
\hline & {$[\mathrm{T}] 180^{1}$} & 0.21 & 19.66 & 39.32 & & \\
\hline \multirow{2}{*}{$\begin{array}{c}\mathrm{Cu} \\
327.393\end{array}$} & {$[\mathrm{~T}]-\mathrm{S}^{2}$} & 1.16 & 33.76 & 135.03 & \multirow{2}{*}{0.0158} & \multirow[b]{2}{*}{19.0} \\
\hline & {$[\mathrm{T}] 180^{1}$} & 1.58 & 54.70 & 109.40 & & \\
\hline \multirow{2}{*}{$\begin{array}{c}\mathrm{Fe} \\
238.204 \\
\end{array}$} & {$[\mathrm{~T}]-\mathrm{S}^{2}$} & 0.59 & 17.23 & 68.92 & \multirow{2}{*}{0.0412} & \multirow{2}{*}{49.5} \\
\hline & {$[\mathrm{T}] 180^{1}$} & 1.81 & 17.40 & 34.80 & & \\
\hline \multirow{2}{*}{$\begin{array}{c}\mathrm{Pb} \\
220.353\end{array}$} & {$[\mathrm{M}]-\mathrm{S}^{1}$} & 0.53 & 117.76 & 235.52 & \multirow[b]{2}{*}{0.0469} & \multirow[b]{2}{*}{94.0} \\
\hline & {$[\mathrm{M}] 180^{\circ}$} & 0.67 & 14.20 & 14.20 & & \\
\hline \multirow{2}{*}{$\begin{array}{c}\mathrm{Cu} \\
327.393 \\
\end{array}$} & {$[\mathrm{M}]-\mathrm{S}^{1}$} & 1.96 & 37.58 & 75.16 & \multirow{2}{*}{0.0109} & \multirow{2}{*}{21.9} \\
\hline & {$[\mathrm{M}] 180^{\circ}$} & 0.99 & 58.74 & 58.74 & & \\
\hline \multirow{2}{*}{$\begin{array}{c}\mathrm{Fe} \\
238.204\end{array}$} & {$[\mathrm{M}]-\mathrm{S}^{1}$} & 2.26 & 20.52 & 41.03 & \multirow{2}{*}{0.0281} & \multirow{2}{*}{56.2} \\
\hline & {$[\mathrm{M}] 180^{\circ}$} & 1.24 & 17.97 & 17.97 & & \\
\hline \multirow{2}{*}{$\begin{array}{c}\mathrm{Ni} \\
231.604\end{array}$} & {$[\mathrm{M}]-\mathrm{S}^{1}$} & 0.56 & 32.87 & 65.75 & \multirow{2}{*}{0.0045} & \multirow{2}{*}{9.10} \\
\hline & {$[\mathrm{M}] 180^{\circ}$} & 1.48 & 59.76 & 59.76 & & \\
\hline $\mathrm{Zn}$ & {$[\mathrm{M}]-\mathrm{S}^{1}$} & 2.23 & 35.99 & 71.98 & \multirow{2}{*}{0.0082} & \multirow{2}{*}{16.5} \\
\hline 206.200 & {$[\mathrm{M}] 180^{\circ}$} & 1.63 & 60.12 & 60.12 & & \\
\hline
\end{tabular}

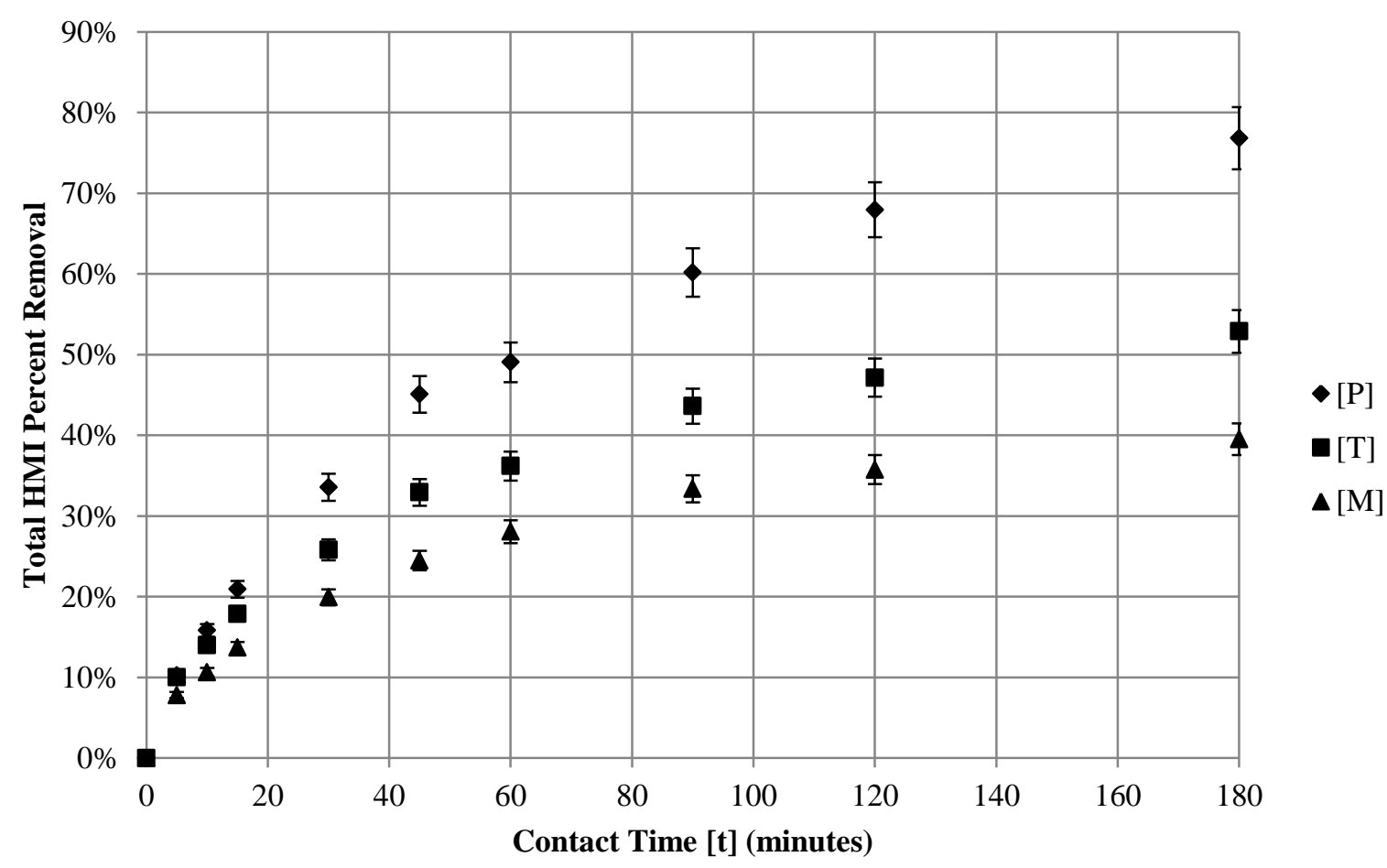

Figure 6.1 Total HMI Percent Removal versus Contact Time 


\subsubsection{Sorption Kinetics}

The selected kinetic relationships are analyzed for all five HMIs and component systems, in both their individual and total combinations. The PSO rate constants and correlation coefficients are summarized in Table 6.4. Based on the linearized form of Equation 6.5, the slope (m) and yintercept (b) values are interpreted to determine the theoretical sorption at equilibrium $\left(\mathrm{q}_{\mathrm{e}}\right)$, overall sorption rate constant $\left(\mathrm{k}_{2}\right)$, and initial sorption rate $(\mathrm{h})$. The IPD rate constants and correlation coefficients are summarized in Table 6.5. Based on Equation 6.9, the slope and yintercept represent the corresponding diffusion rate constant $\left(\mathrm{k}_{\mathrm{P}}\right)$ and boundary constant $(\mathrm{C})$, respectively.

An impressive performance is observed in the correlation coefficient (CC) for both models of all the component system combinations. For the PSO model, 64.1\% of the data falls within 0.95-1.0 CC range (excellent), while $84.6 \%$ has a CC of over 0.90 (good), and $87.2 \%$ has a CC over 0.85 (acceptable). For the IPD model, Equation 6.9 is applied in two scenarios with respect to the origin. Good linearization of the data is observed for the initial phase of the reaction, in accordance with expected behaviour if intra-particle diffusion is the rate-determining step (Ho and McKay, 1998b; Zolgharnein and Shahmoradi, 2010). For the first scenario (at origin ${ }^{\mathrm{A}}$ ), $64.1 \%$ of the data falls within the excellent range, while $82.1 \%$ within the good range and $87.2 \%$ within the acceptable range. For the second scenario (deviation from origin ${ }^{\mathrm{B}}$ ), $79.5 \%$ of the data falls within the excellent range, while $94.9 \%$ within the good range. It is important to note that the boundary constant $(\mathrm{C})$ values observed are very small and considered negligible, with $35.9 \%$ of the data showing a negative value with no physical meaning (Fierro et al., 2008; Olu-Owolabi et al., 2014). When comparing the diffusion rate constant $\left(\mathrm{k}_{\mathrm{P}}\right)$ values, an average of positive $\mathrm{C}$ values differs by only $0.0004 \mathrm{meq} / \mathrm{g} \cdot \mathrm{min}$ between the two scenarios, demonstrating that the slopes $\left(\mathrm{k}_{\mathrm{P}}\right)$ are very similar. Although a stronger $\mathrm{CC}$ is observed when deviation from the origin, it may be stated with confidence that intra-particle diffusion is the leading diffusion mechanism to represent the sorption process. Of the excellent CC range, the data correlated at 0.99 or above to unity is only $4.00 \%$ ( 1 of 25 ) by the IPD model while $48.0 \%$ (12 of 25 ) by the PSO model. As

observed by Bektas and Kara (2004), a stronger correlation is observed by the PSO kinetic rate expression. 
Table 6.4 Pseudo-Second-Order (PSO) Data

\begin{tabular}{|c|c|c|c|c|c|c|}
\hline SYSTEM & $\mathbf{m}=\left(\frac{1}{a}\right)$ & $\mathbf{b}=\left(\frac{1}{k^{2 \mathbf{e}^{2}}}\right)$ & $\mathrm{CC}$ & $\begin{array}{c}\text { Equilibrium } \\
\text { Sorption }\end{array}$ & $\begin{array}{c}\begin{array}{c}\text { Sorption Rate } \\
\text { Constant }\end{array} \\
\end{array}$ & $\begin{array}{c}\text { Initial } \\
\text { Sorption Rate } \\
\end{array}$ \\
\hline & & & & $\mathrm{q}_{\mathrm{e}}(\mathrm{meq} / \mathrm{g})$ & $\mathrm{k}_{2}(\mathrm{~g} / \mathrm{meq} \cdot \min )$ & $\mathrm{h}=\mathrm{k}_{2} \mathrm{q}_{\mathrm{e}}^{2}(\mathrm{meq} / \mathrm{g} \cdot \mathrm{min})$ \\
\hline$[\mathbf{P}]$ & 4.098 & 217.01 & 0.9926 & 0.244 & 0.077 & 0.0046 \\
\hline$[\mathrm{C}]$ & 15.750 & 836.09 & 0.9291 & 0.063 & 0.297 & 0.0012 \\
\hline$[\mathbf{F}]$ & 11.872 & 419.08 & 0.9708 & 0.084 & 0.336 & 0.0024 \\
\hline [N] & 34.919 & 739.14 & 0.9806 & 0.029 & 1.650 & 0.0014 \\
\hline [Z] & 15.237 & 1106.10 & 0.9147 & 0.066 & 0.210 & 0.0009 \\
\hline [D-PC]-Pb & 7.474 & 299.43 & 0.9941 & 0.134 & 0.187 & 0.0033 \\
\hline [D-PC]-Cu & 55.217 & 2840.90 & 0.9282 & 0.018 & 1.073 & 0.0004 \\
\hline [D-PC] & 6.596 & 269.78 & 0.9906 & 0.152 & 0.161 & 0.0037 \\
\hline$[\mathrm{D}-\mathrm{PF}]-\mathrm{Pb}$ & 7.692 & 448.41 & 0.9904 & 0.130 & 0.132 & 0.0022 \\
\hline$[\mathrm{D}-\mathrm{PF}]-\mathrm{Fe}$ & 18.375 & 727.45 & 0.9703 & 0.054 & 0.464 & 0.0014 \\
\hline [D-PF $]$ & 5.489 & 275.71 & 0.9837 & 0.182 & 0.109 & 0.0036 \\
\hline$[\mathrm{D}-\mathrm{PN}]-\mathrm{Pb}$ & 7.465 & 284.48 & 0.9957 & 0.134 & 0.196 & 0.0035 \\
\hline [D-PN]-Ni & 109.750 & 3126.00 & 0.5378 & 0.009 & 3.853 & 0.0003 \\
\hline [D-PN] & 6.976 & 254.36 & 0.9914 & 0.143 & 0.191 & 0.0039 \\
\hline$[\mathrm{D}-\mathrm{PZ}]-\mathrm{Pb}$ & 7.436 & 293.22 & 0.9973 & 0.134 & 0.189 & 0.0034 \\
\hline [D-PZ]-Zn & 40.653 & 5898.50 & 0.4358 & 0.025 & 0.280 & 0.0002 \\
\hline [D-PZ] & 6.534 & 280.74 & 0.9946 & 0.153 & 0.152 & 0.0036 \\
\hline$[\mathrm{D}-\mathrm{CF}]-\mathrm{Cu}$ & 21.169 & 2683.00 & 0.9629 & 0.047 & 0.167 & 0.0004 \\
\hline$[\mathrm{D}-\mathrm{CF}]-\mathrm{Fe}$ & 16.655 & 581.48 & 0.9750 & 0.060 & 0.477 & 0.0017 \\
\hline [D-CF $]$ & 10.297 & 488.73 & 0.9703 & 0.097 & 0.217 & 0.0020 \\
\hline$[\mathrm{D}-\mathrm{CN}]-\mathrm{Cu}$ & 21.936 & 1271.30 & 0.9453 & 0.046 & 0.379 & 0.0008 \\
\hline$[\mathrm{D}-\mathrm{CN}]-\mathrm{Ni}$ & 72.008 & 1686.80 & 0.9498 & 0.014 & 3.074 & 0.0006 \\
\hline [D-CN] & 17.329 & 747.04 & 0.9471 & 0.058 & 0.402 & 0.0013 \\
\hline [D-FZ]-Fe & 16.438 & 502.40 & 0.9778 & 0.061 & 0.538 & 0.0020 \\
\hline [D-FZ]-Zn & 28.869 & 3101.00 & 0.6387 & 0.035 & 0.269 & 0.0003 \\
\hline [D-FZ] & 11.140 & 451.04 & 0.9554 & 0.090 & 0.275 & 0.0022 \\
\hline [D-NZ]-Ni & 133.600 & 999.38 & 0.9731 & 0.007 & 17.860 & 0.0010 \\
\hline [D-NZ]-Zn & 19.459 & 3808.80 & 0.3642 & 0.051 & 0.099 & 0.0003 \\
\hline [D-NZ] & 24.852 & 1452.00 & 0.9113 & 0.040 & 0.425 & 0.0007 \\
\hline$[\mathrm{T}]-\mathrm{Pb}$ & 11.194 & 394.10 & 0.9980 & 0.089 & 0.318 & 0.0025 \\
\hline$[\mathrm{T}]-\mathrm{Cu}$ & 48.524 & 3252.90 & 0.9141 & 0.021 & 0.724 & 0.0003 \\
\hline$[\mathrm{T}]-\mathrm{Fe}$ & 21.496 & 721.14 & 0.9807 & 0.047 & 0.641 & 0.0014 \\
\hline$[\mathbf{T}]$ & 6.438 & 237.29 & 0.9918 & 0.155 & 0.175 & 0.0042 \\
\hline$[\mathrm{M}]-\mathrm{Pb}$ & 18.593 & 465.67 & 0.9990 & 0.054 & 0.742 & 0.0021 \\
\hline$[\mathrm{M}]-\mathrm{Cu}$ & 58.274 & 6429.20 & 0.8600 & 0.017 & 0.528 & 0.0002 \\
\hline$[\mathrm{M}]-\mathrm{Fe}$ & 31.695 & 989.38 & 0.9830 & 0.032 & 1.015 & 0.0010 \\
\hline$[\mathrm{M}]-\mathrm{Ni}$ & 190.820 & 5086.10 & 0.9747 & 0.005 & 7.159 & 0.0002 \\
\hline$[\mathrm{M}]-\mathrm{Zn}$ & -51.894 & $2,5482.00$ & 0.1723 & -0.019 & 0.106 & 0.0000 \\
\hline$[\mathbf{M}]$ & 8.630 & 303.14 & 0.9931 & 0.116 & 0.246 & 0.0033 \\
\hline
\end{tabular}


Table 6.5 Intra-Particle Diffusion (IPD) Data

\begin{tabular}{|c|c|c|c|c|c|c|}
\hline SYSTEM & $\begin{array}{c}\begin{array}{c}\text { Diffusion Rate } \\
\text { Constant }^{\mathrm{A}}\end{array} \\
\mathrm{k}_{\mathrm{p}}\left(\mathrm{meq} / \mathrm{g} \cdot \mathrm{min}^{1 / 2}\right)\end{array}$ & $\mathbf{C C}^{\mathrm{A}}$ & SYSTEM & $\begin{array}{c}\begin{array}{c}\text { Diffusion Rate } \\
\text { Constant }^{\mathrm{B}}\end{array} \\
\mathrm{k}_{\mathrm{P}}\left(\mathrm{meq} / \mathrm{g} \cdot \mathrm{min}^{1 / 2}\right)\end{array}$ & $\begin{array}{c}\begin{array}{c}\text { Boundary } \\
\text { Constant }^{\text {B }}\end{array} \\
\mathrm{C}(\mathrm{meg} / \mathrm{g})\end{array}$ & $\mathbf{C C}^{\mathrm{B}}$ \\
\hline$[\mathbf{P}]$ & 0.015 & 0.9818 & {$[\mathbf{P}]$} & 0.016 & -0.004 & 0.9828 \\
\hline [C] & 0.004 & 0.9882 & [C] & 0.004 & -0.000 & 0.9883 \\
\hline$[\mathbf{F}]$ & 0.006 & 0.9548 & {$[\mathbf{F}]$} & 0.005 & 0.006 & 0.9747 \\
\hline [N] & 0.002 & 0.6930 & [N] & 0.002 & 0.007 & 0.9629 \\
\hline [Z] & 0.004 & 0.9729 & [Z] & 0.004 & -0.003 & 0.9846 \\
\hline$[\mathrm{D}-\mathrm{PC}]-\mathrm{Pb}$ & 0.009 & 0.9597 & [D-PC]-Pb & 0.009 & 0.004 & 0.9627 \\
\hline$[\mathrm{D}-\mathrm{PC}]-\mathrm{Cu}$ & 0.001 & 0.9658 & {$[\mathrm{D}-\mathrm{PC}]-\mathrm{Cu}$} & 0.001 & 0.000 & 0.9669 \\
\hline [D-PC] & 0.010 & 0.9662 & [D-PC] & 0.010 & 0.004 & 0.9690 \\
\hline [D-PF]-Pb & 0.008 & 0.9790 & [D-PF]-Pb & 0.008 & -0.004 & 0.9827 \\
\hline [D-PF]-Fe & 0.004 & 0.9860 & {$[\mathrm{D}-\mathrm{PF}]-\mathrm{Fe}$} & 0.003 & 0.003 & 0.9951 \\
\hline [D-PF] & 0.012 & 0.9873 & [D-PF $]$ & 0.012 & -0.001 & 0.9876 \\
\hline$[\mathrm{D}-\mathrm{PN}]-\mathrm{Pb}$ & 0.009 & 0.9569 & {$[\mathrm{D}-\mathrm{PN}]-\mathrm{Pb}$} & 0.009 & 0.005 & 0.9622 \\
\hline [D-PN]-Ni & 0.001 & 0.4479 & [D-PN]-Ni & 0.001 & 0.002 & 0.5521 \\
\hline [D-PN] & 0.010 & 0.9527 & [D-PN] & 0.009 & 0.007 & 0.9616 \\
\hline [D-PZ]-Pb & 0.009 & 0.9542 & [D-PZ]-Pb & 0.009 & 0.004 & 0.9576 \\
\hline [D-PZ]-Zn & 0.001 & 0.9088 & [D-PZ]-Zn & 0.001 & -0.002 & 0.9378 \\
\hline [D-PZ] & 0.010 & 0.9639 & [D-PZ] & 0.010 & 0.003 & 0.9649 \\
\hline$[\mathrm{D}-\mathrm{CF}]-\mathrm{Cu}$ & 0.002 & 0.9523 & {$[\mathrm{D}-\mathrm{CF}]-\mathrm{Cu}$} & 0.002 & -0.004 & 0.9933 \\
\hline$[\mathrm{D}-\mathrm{CF}]-\mathrm{Fe}$ & 0.004 & 0.9666 & {$[\mathrm{D}-\mathrm{CF}]-\mathrm{Fe}$} & 0.004 & 0.005 & 0.9902 \\
\hline [D-CF $]$ & 0.006 & 0.9923 & [D-CF $]$ & 0.006 & 0.001 & 0.9925 \\
\hline$[\mathrm{D}-\mathrm{CN}]-\mathrm{Cu}$ & 0.003 & 0.9888 & {$[\mathrm{D}-\mathrm{CN}]-\mathrm{Cu}$} & 0.003 & -0.001 & 0.9915 \\
\hline$[\mathrm{D}-\mathrm{CN}]-\mathrm{Ni}$ & 0.001 & 0.6540 & {$[\mathrm{D}-\mathrm{CN}]-\mathrm{Ni}$} & 0.001 & 0.003 & 0.9089 \\
\hline [D-CN] & 0.004 & 0.9795 & [D-CN] & 0.004 & 0.002 & 0.9843 \\
\hline$[\mathrm{D}-\mathrm{FZ}]-\mathrm{Fe}$ & 0.005 & 0.9332 & [D-FZ]-Fe & 0.004 & 0.007 & 0.9906 \\
\hline [D-FZ]-Zn & 0.002 & 0.9303 & [D-FZ]-Zn & 0.002 & -0.002 & 0.9494 \\
\hline [D-FZ] & 0.006 & 0.9777 & [D-FZ] & 0.006 & 0.005 & 0.9907 \\
\hline [D-NZ]-Ni & 0.001 & -0.2510 & [D-NZ]-Ni & 0.000 & 0.003 & 0.6264 \\
\hline [D-NZ]-Zn & 0.002 & 0.9348 & [D-NZ]-Zn & 0.002 & -0.004 & 0.9801 \\
\hline [D-NZ] & 0.002 & 0.9605 & [D-NZ] & 0.002 & -0.000 & 0.9605 \\
\hline$[\mathrm{T}]-\mathrm{Pb}$ & 0.006 & 0.9434 & {$[\mathrm{~T}]-\mathrm{Pb}$} & 0.006 & 0.005 & 0.9536 \\
\hline$[\mathrm{T}]-\mathrm{Cu}$ & 0.001 & 0.9747 & {$[\mathrm{~T}]-\mathrm{Cu}$} & 0.001 & -0.001 & 0.9826 \\
\hline$[\mathrm{T}]-\mathrm{Fe}$ & 0.003 & 0.9622 & {$[\mathrm{~T}]-\mathrm{Fe}$} & 0.003 & 0.004 & 0.9900 \\
\hline$[\mathrm{T}]$ & 0.011 & 0.9668 & {$[\mathbf{T}]$} & 0.010 & 0.008 & 0.9761 \\
\hline$[\mathrm{M}]-\mathrm{Pb}$ & 0.004 & 0.8584 & {$[\mathrm{M}]-\mathrm{Pb}$} & 0.004 & 0.007 & 0.9130 \\
\hline$[\mathrm{M}]-\mathrm{Cu}$ & 0.001 & 0.9473 & {$[\mathrm{M}]-\mathrm{Cu}$} & 0.001 & -0.001 & 0.9851 \\
\hline$[\mathrm{M}]-\mathrm{Fe}$ & 0.002 & 0.9465 & {$[\mathrm{M}]-\mathrm{Fe}$} & 0.002 & 0.003 & 0.9886 \\
\hline$[\mathrm{M}]-\mathrm{Ni}$ & 0.000 & 0.8566 & {$[\mathrm{M}]-\mathrm{Ni}$} & 0.000 & 0.001 & 0.9251 \\
\hline$[\mathrm{M}]-\mathrm{Zn}$ & 0.001 & 0.8479 & {$[\mathrm{M}]-\mathrm{Zn}$} & 0.001 & -0.002 & 0.9720 \\
\hline [M] & 0.008 & 0.9585 & {$[\mathbf{M}]$} & 0.008 & 0.007 & 0.9713 \\
\hline
\end{tabular}


A significantly low correlation is observed for the singular uptake of the $\mathrm{Zn}^{2+}$ ion in the [DPZ], [D-FZ], [D-NZ], and [M] component systems when observed by the PSO model. The $\mathrm{Ni}^{2+}$ ion in the $[\mathrm{N}],[\mathrm{D}-\mathrm{PN}],[\mathrm{D}-\mathrm{CN}]$ and [D-NZ] component systems also demonstrates a weaker correlation in its singular uptake when observed by the IPD model. However, due to zeolite's lower selectivity to these ions, the overall uptake in each combined component system is not significantly affected by these incurred outliers. Based on the initial sorption rate determined by the PSO kinetic model given in Table 6.4, the single-component system of lead $\left(\mathrm{Pb}^{2+}\right)[\mathrm{P}]$ achieves the greatest rate at $0.0046 \mathrm{meq} / \mathrm{g} \cdot \mathrm{min}$ among the HMI investigated. In the dualcomponent systems, the overall uptake of $\mathrm{HMI}$ containing $\mathrm{Pb}^{2+}$ was on average 0.0037 $\mathrm{meq} / \mathrm{g} \cdot \mathrm{min}$. For the dual-component systems without $\mathrm{Pb}^{2+}$, the uptake rate was on average $0.0016 \mathrm{meq} / \mathrm{g} \cdot \mathrm{min}$. The initial sorption rates in the triple- and multi-component systems were observed as 0.0042 and $0.0033 \mathrm{meq} / \mathrm{g} \cdot \mathrm{min}$, respectively. This kinetic analysis has demonstrated that the initial uptake rate of total HMIs is most improved with the presence of lead.

Although the Elovich equation was not selected as the primary model of consideration to represent the reaction-type chemisorption relationship associated with the sorption process, the comparison between initial sorption rates is a valued discussion. In the kinetic analysis of lead removal from aqueous solution by natural clinoptilolite by Gunay et al. (2007), the Elovich equation produced trends that the initial sorption rate (related to chemisorption) increased with an increase in initial concentration, while the $\beta$ constant (related to surface coverage) decreased with an increase in initial concentration and increased with an increase in mass of sorbent (site availability). With reference to Table 6.6 of this study, the overall influence of the total HMI removal on each component system is consistent between each model, with the initial sorption rate of $\alpha$ being on average $0.0039 \mathrm{meq} / \mathrm{g} \cdot \mathrm{min}$ greater than that of $\mathrm{h}$ (Largitte and Pasquier, 2016). The data shows a stronger correlation with the PSO model compared to that of the Elovich Equation; validating the model selection rationale of this study. 
Table 6.6 Pseudo-Second-Order (PSO) and Elovich Data Comparison

\begin{tabular}{|c|c|c|c|c|c|c|c|}
\hline \multirow{3}{*}{ SYSTEM } & \multicolumn{2}{|r|}{ PSO } & \multicolumn{5}{|c|}{ Elovich } \\
\hline & \multirow{2}{*}{$\mathrm{CC}$} & $\begin{array}{c}\text { Initial } \\
\text { Sorption Rate }\end{array}$ & \multirow{2}{*}{$\mathrm{CC}$} & \multirow{2}{*}{$\mathbf{m}=\left(\frac{\mathbf{1}}{\boldsymbol{\beta}}\right)$} & \multirow{2}{*}{$b=\left(\frac{\mathbf{1}}{\boldsymbol{\beta}}\right)[\ln (\boldsymbol{\alpha} \boldsymbol{\beta})]$} & $\begin{array}{c}\text { Elovich } \\
\text { Constant }\end{array}$ & $\begin{array}{c}\text { Initial } \\
\text { Sorption Rate }\end{array}$ \\
\hline & & $\begin{array}{c}\mathrm{h}=\mathrm{k}_{2} \mathrm{q}_{\mathrm{e}}^{2} \\
(\mathrm{meq} / \mathrm{g} \cdot \mathrm{min})\end{array}$ & & & & $\begin{array}{c}\beta \\
\text { (g/meq) } \\
\end{array}$ & $\begin{array}{c}\alpha \\
(\mathrm{meq} / \mathrm{g} \cdot \min ) \\
\end{array}$ \\
\hline$[\mathbf{P}]$ & 0.9926 & 0.0046 & 0.9742 & 0.0486 & -0.0697 & 20.58 & 0.0116 \\
\hline [C] & 0.9291 & 0.0012 & 0.9104 & 0.0118 & -0.0151 & 84.75 & 0.0033 \\
\hline$[\mathbf{F}]$ & 0.9708 & 0.0024 & 0.9606 & 0.0165 & -0.0162 & 60.61 & 0.0062 \\
\hline [N] & 0.9806 & 0.0014 & 0.9614 & 0.0049 & -0.0002 & 204.08 & 0.0047 \\
\hline [Z] & 0.9147 & 0.0009 & 0.9208 & 0.012 & -0.0188 & 83.33 & 0.0025 \\
\hline [D-PC] & 0.9906 & 0.0037 & 0.9730 & 0.0309 & -0.0382 & 32.36 & 0.0090 \\
\hline [D-PF] & 0.9837 & 0.0036 & 0.9636 & 0.0361 & -0.0496 & 27.70 & 0.0091 \\
\hline [D-PN] & 0.9914 & 0.0039 & 0.9767 & 0.0294 & -0.0336 & 34.01 & 0.0094 \\
\hline [D-PZ] & 0.9946 & 0.0036 & 0.9794 & 0.0315 & -0.0409 & 31.75 & 0.0086 \\
\hline [D-CF $]$ & 0.9703 & 0.0020 & 0.9564 & 0.0189 & -0.0243 & 52.91 & 0.0052 \\
\hline [D-CN] & 0.9471 & 0.0013 & 0.9165 & 0.0109 & -0.012 & 91.74 & 0.0036 \\
\hline [D-FZ] & 0.9554 & 0.0022 & 0.9174 & 0.0166 & -0.0162 & 60.24 & 0.0063 \\
\hline [D-NZ] & 0.9113 & 0.0007 & 0.9164 & 0.0075 & -0.0099 & 133.33 & 0.0020 \\
\hline [T]-Pb & 0.9980 & 0.0025 & 0.9880 & 0.0186 & -0.0214 & 53.76 & 0.0059 \\
\hline [T] & 0.9918 & 0.0042 & 0.9809 & 0.0314 & -0.0354 & 31.85 & 0.0102 \\
\hline$[\mathbf{M}]-\mathbf{P b}$ & 0.9990 & 0.0021 & 0.9869 & 0.0113 & -0.0097 & 88.50 & 0.0048 \\
\hline [M] & 0.9931 & 0.0033 & 0.9815 & 0.0236 & -0.0257 & 42.37 & 0.0079 \\
\hline
\end{tabular}

The trendlines' equations displayed in Table 6.4 and Table 6.5 are applied to the kinetic models for the total HMI uptake of the various component systems, and shown for visual comparisons in Figures 6.2 to 6.4. Figure 6.5 presents the uptake $\left(\mathrm{q}_{\mathrm{t}}\right)$ of all five HMIs in the [M] multi-component system over the three-hour contact period, with both kinetic models applied in linearized form based on Equation 6.6 and excluding the boundary constant of Equation 6.9. It is observed that even for this most complicated system containing all five HMIs $[\mathrm{M}]$, it is evident that a stronger correlation, at a CC of 0.9931, is achieved with the PSO rate model. 


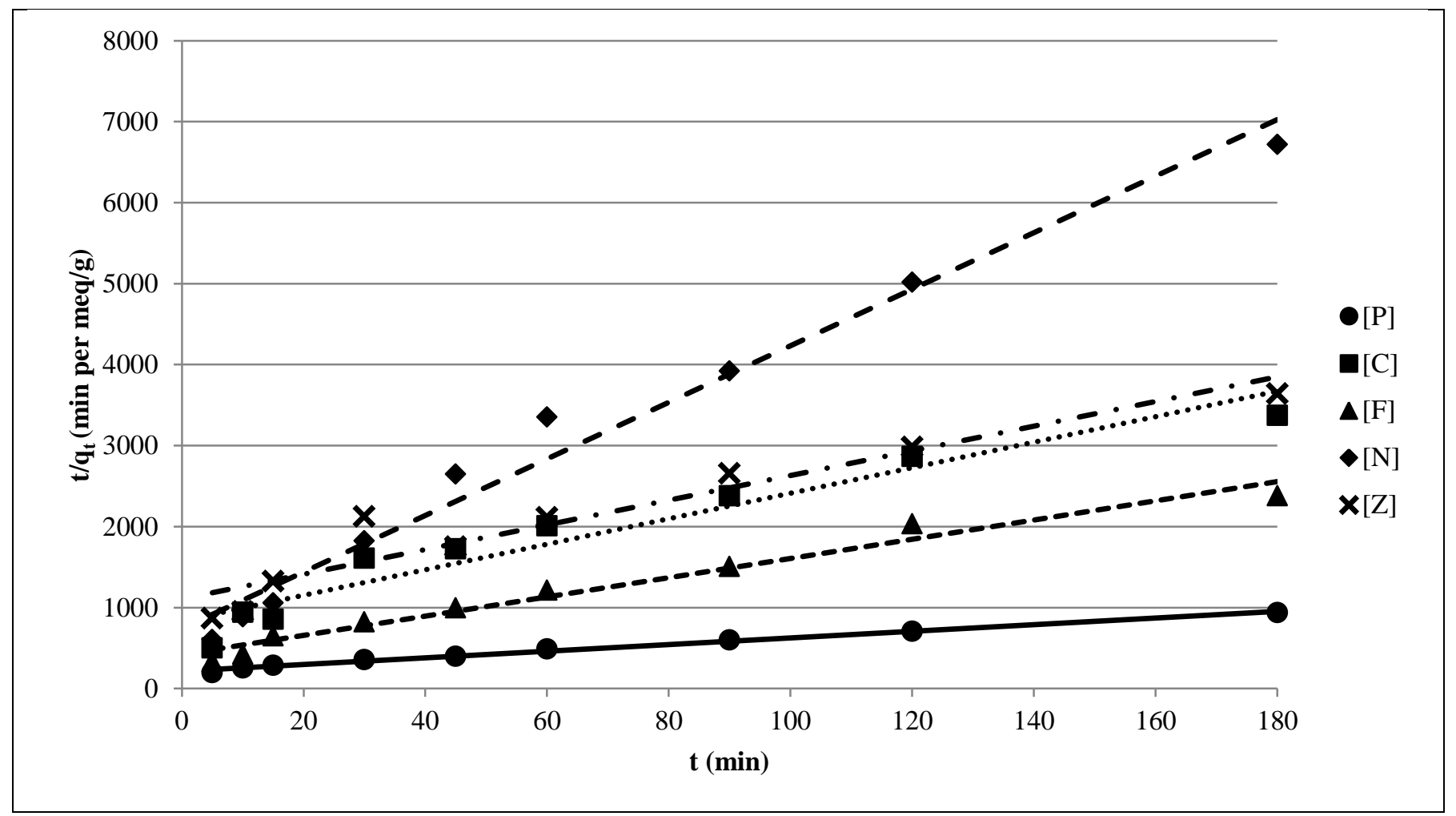

a.

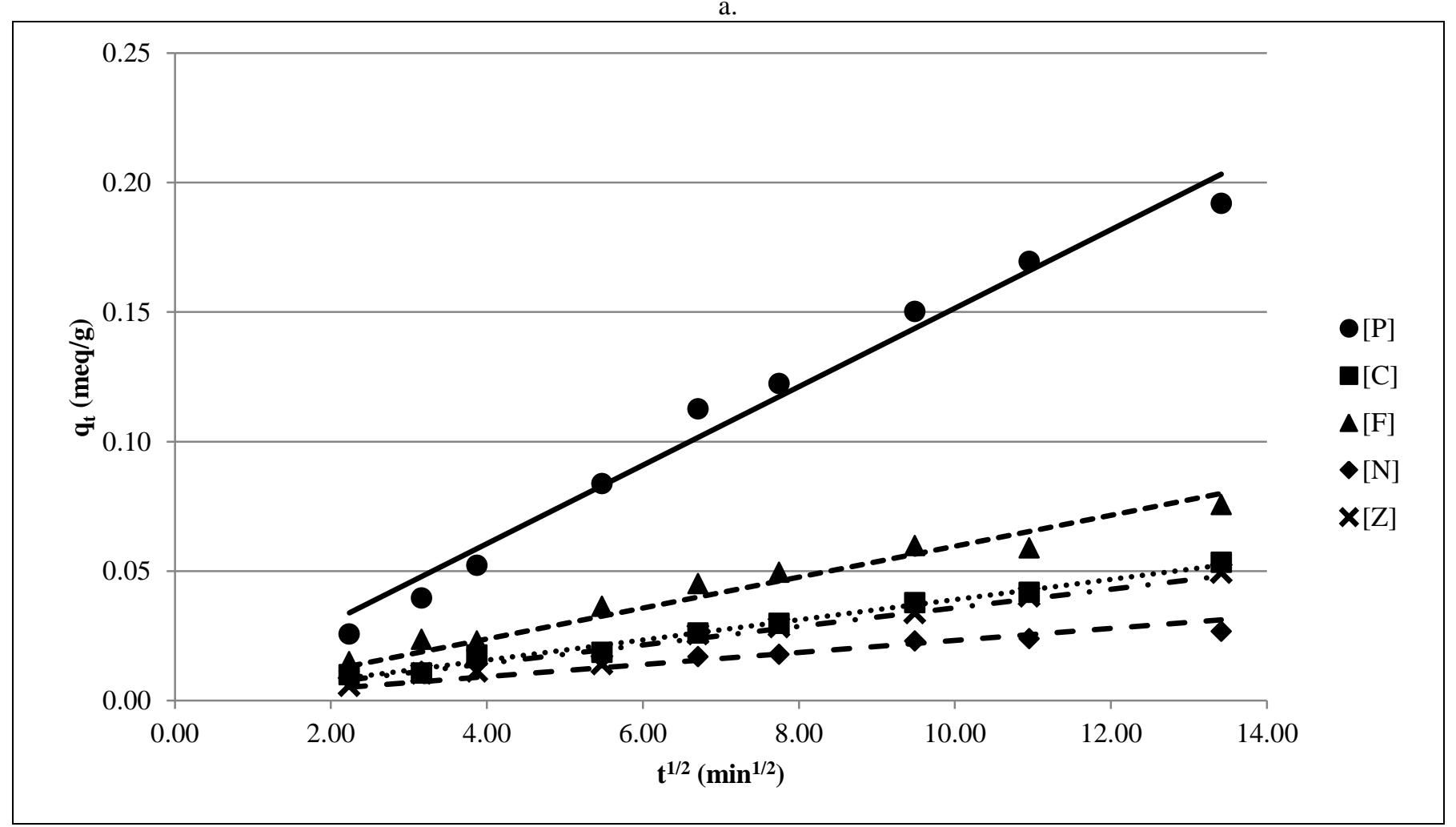

b.

Figure 6.2 Single-Component System Kinetic Model Plots

a. pseudo-second-order; b. intra-particle diffusion 


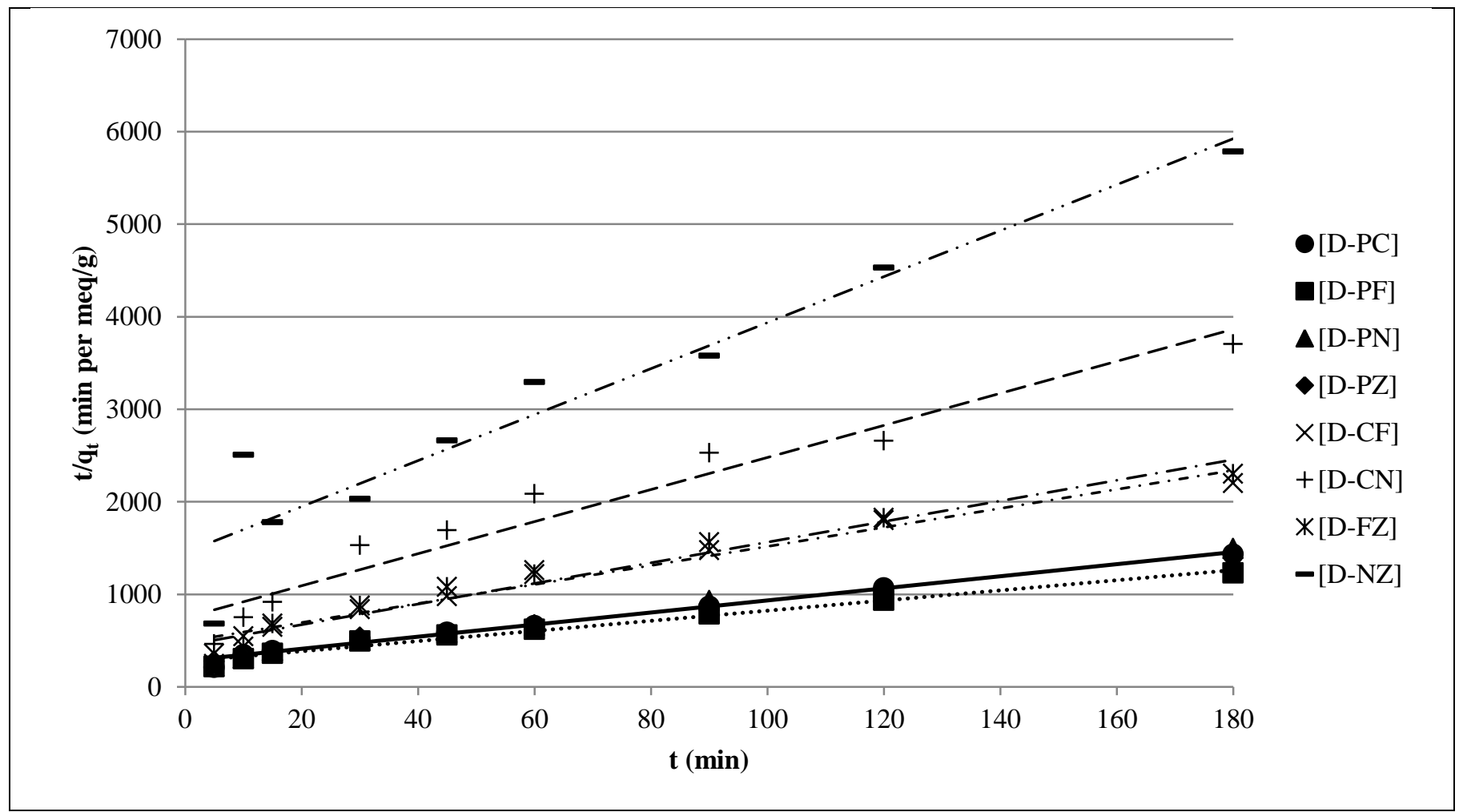

a.

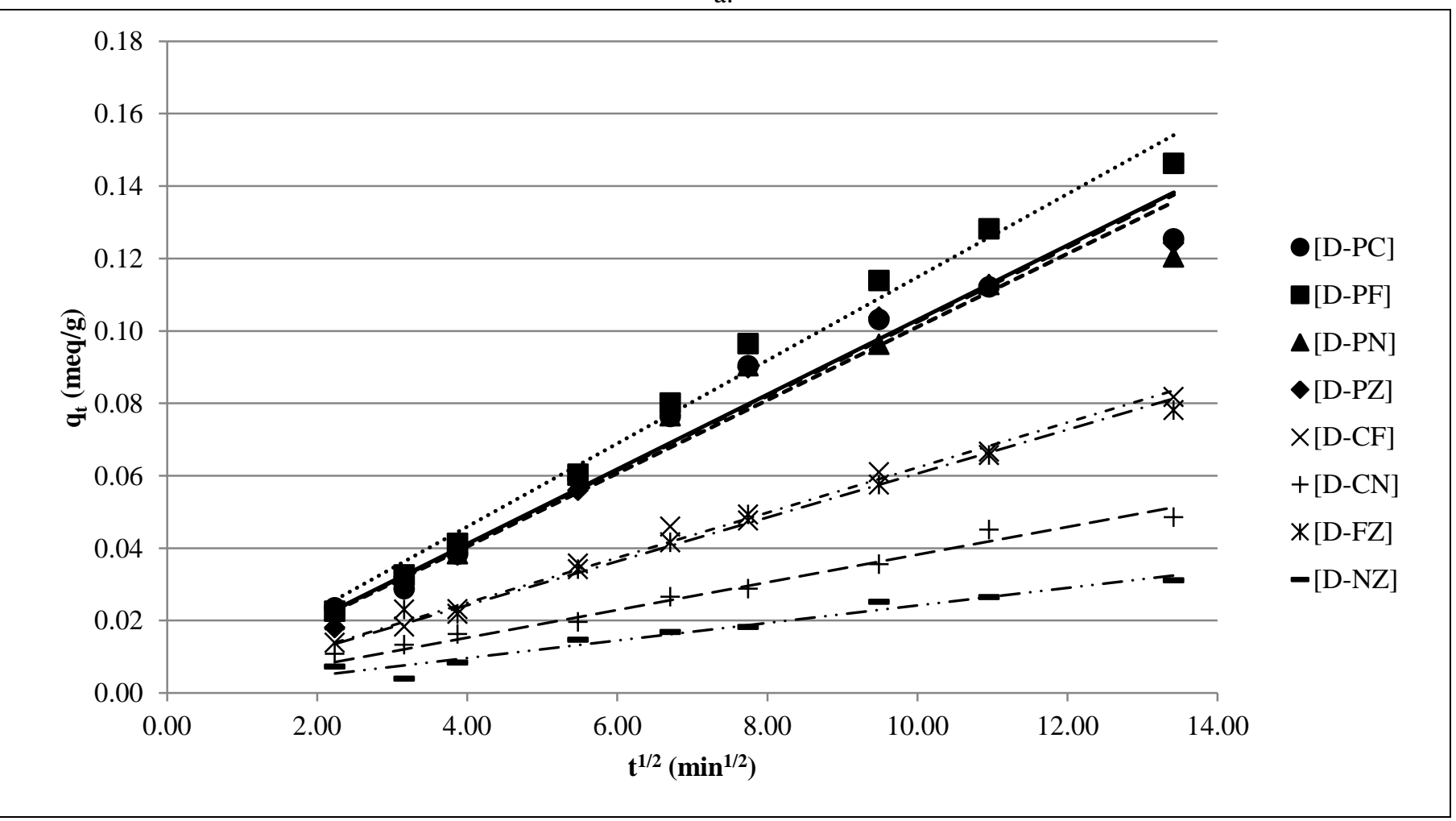

Figure 6.3 Dual-Component System Kinetic Model Plots

a. pseudo-second-order; b. intra-particle diffusion 


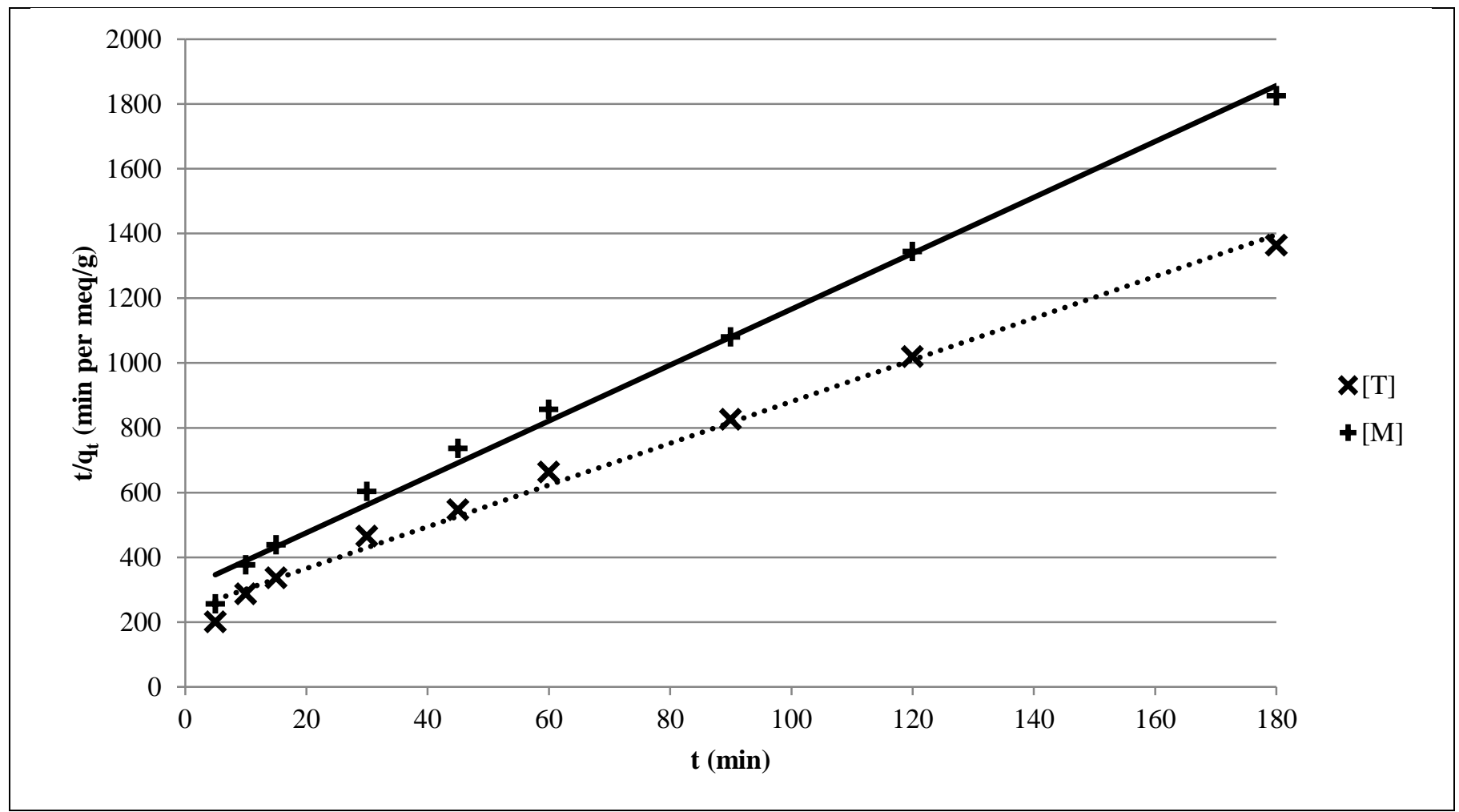

a.

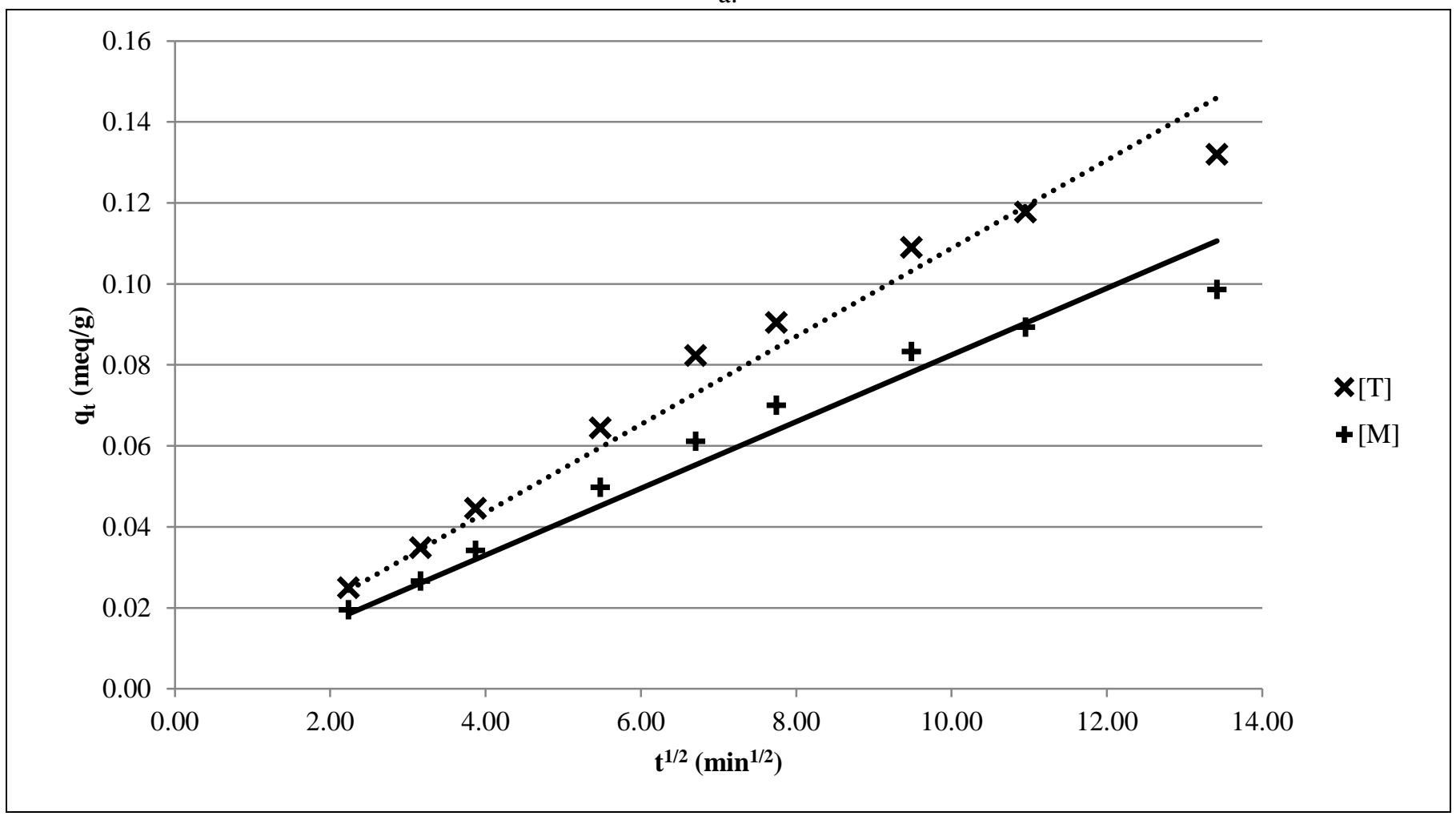

Figure 6.4 Triple- and Multi-Component System Kinetic Model Plots

a. pseudo-second-order; b. intra-particle diffusion 


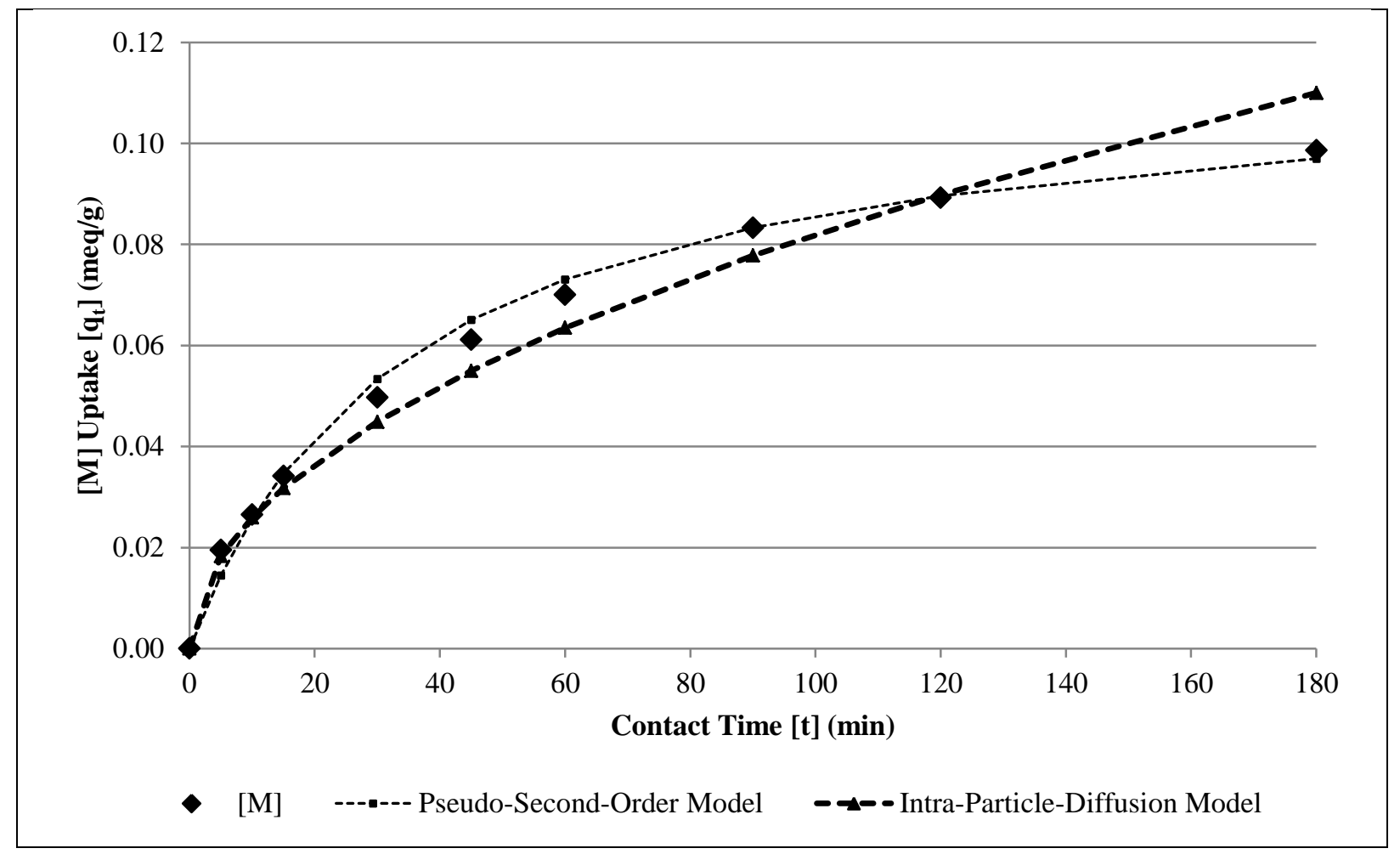

Figure 6.5 Multi-component System - Uptake Over Time alongside Kinetic Models

The data presented in Table 6.7 provides compelling trends into the removal efficiency and the zeolite's selectivity to the five HMIs investigated in this study. When comparing the total HMI uptake at 3 contact hours to the theoretical equilibrium values generated by the PSO model of each system, an average of $83.5 \%$ of the equilibrium uptake is achieved. In the singlecomponent systems, the uptake of $[\mathrm{P}]$ is highest, followed by $[\mathrm{F}],[\mathrm{C}],[\mathrm{Z}]$, and $[\mathrm{N}]$. Only $78.7 \%$ of $[\mathrm{P}]$ at equilibrium is achieved, whereas $93.5 \%$ of $[\mathrm{N}]$ at equilibrium is observed. At the opposite end of removal efficiency, there is this significant difference in overall uptake. In the dual-component systems, the $[\mathrm{D}-\mathrm{PF}]$ is the greatest uptake and the [D-NZ] is the least. The overall HMI uptake is increased by an average of approximately $0.0778 \mathrm{meq} / \mathrm{g}$ or $152 \%$ when combined with $\mathrm{Pb}^{2+}$; when comparing $[\mathrm{C}],[\mathrm{F}],[\mathrm{N}]$, and [Z] to [D-PC], [D-PF], [D-PN] and [D$\mathrm{PZ}$, respectively. For the dual-systems without $\mathrm{Pb}^{2+}$, the presence of $\mathrm{Fe}^{3+}$ improves the uptake in [D-CF] and [D-FZ] by 53.2 and $58.1 \%$, respectively; the $\mathrm{Ni}^{2+}$ hinders the overall uptake in [D$\mathrm{CN}$ ] and [D-NZ] by 9.00 and 37.1\%, respectively. The combination of lead, copper and iron in the triple-component system increases the [D-PC] and [D-CF] by 5.20 and $61.5 \%$, respectively; but the inclusion of $\mathrm{Cu}^{2+}$ decreases the $[\mathrm{D}-\mathrm{PF}]$ by $9.80 \%$. Once again, the $\mathrm{Pb}^{2+}$ ion has the greatest improvement to the overall uptake of total HMI of a given system. In the multi- 
component system, the introduction of $\mathrm{Ni}^{2+}$ and $\mathrm{Zn}^{2+}$ impedes on the total HMI uptake in the triple system [T] by $25 \%$ for both the experimental and theoretical equilibrium data. This demonstrates that each HMI impacts the zeolite's ability to sorb those co-ions in solution.

As reported in Table 6.7, the zeolite removal efficiency order based on the experimental and theoretically derived equilibrium HMI uptake data is observed as $\mathrm{Pb}^{2+}>>\mathrm{Fe}^{3+}>\mathrm{Cu}^{2+}>\mathrm{Zn}^{2+}>$ $\mathrm{Ni}^{2+}$. This selectivity trend is also observed in other experimental findings, including Wang and Peng (2010), and Inglezakis et al. (2002; 2003). Current research on the HMI sorption capacity of natural zeolites and other sorbent materials is summarized in Table 6.8. Accordingly, the natural zeolite employed in this study compares favourably and within trend based on the major findings of current research endeavours.

Table 6.7 Experimental Total and Theoretical Equilibrium HMI Uptake System Observations

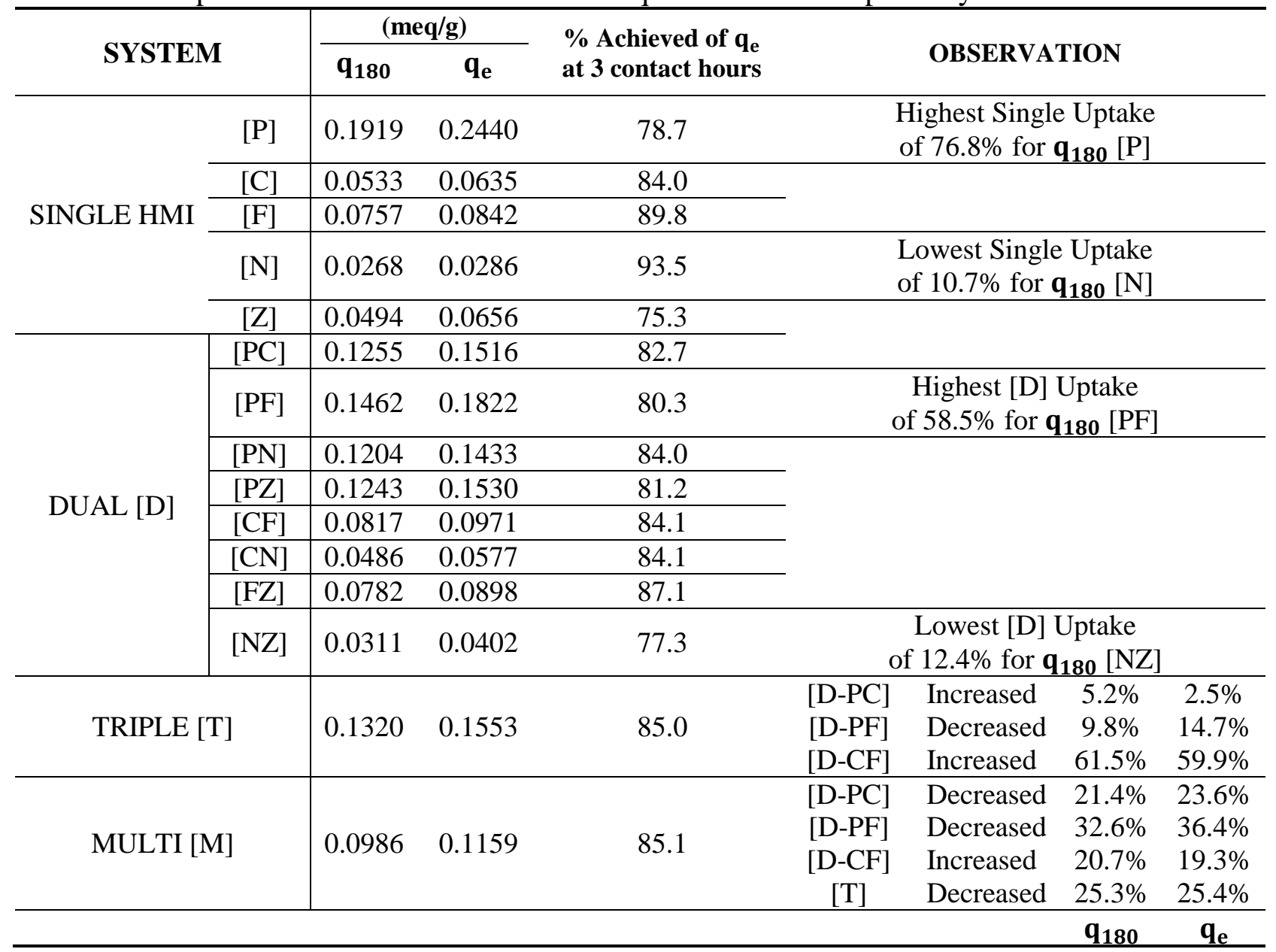


Table 6.8 The Sorption Trends of Heavy Metallic Ions on Various Sorbent Materials

\begin{tabular}{|c|c|c|c|}
\hline Sorbent Material & HMI & Sorption Trends & Reference \\
\hline $\begin{array}{l}\text { American } \\
\text { 85-95\% Clinoptilolite }\end{array}$ & $\begin{array}{l}\mathrm{Pb}^{2+} \\
\mathrm{Fe}^{3+} \\
\mathrm{Cu}^{2+} \\
\mathrm{Zn}^{2+} \\
\mathrm{Ni}^{2+}\end{array}$ & 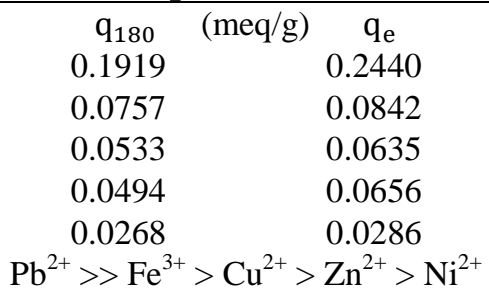 & This Study \\
\hline $\begin{array}{c}\text { Brazilian } \\
\text { Zeolite (Scolecite) }\end{array}$ & $\begin{array}{l}\mathrm{Cu}^{2+} \\
\mathrm{Zn}^{2+} \\
\mathrm{Pb}^{2+} \\
\mathrm{Ni}^{2+} \\
\mathrm{Co}^{2+} \\
\mathrm{Cd}^{2+}\end{array}$ & $\begin{array}{c}130 \mu \mathrm{eq} / \mathrm{g} \\
64 \mu \mathrm{eq} / \mathrm{g} \\
56 \mu \mathrm{eq} / \mathrm{g} \\
31 \mu \mathrm{eq} / \mathrm{g} \\
7.8 \mu \mathrm{eq} / \mathrm{g} \\
3.2 \mu \mathrm{eq} / \mathrm{g} \\
\mathrm{Cu}^{2+}>>\mathrm{Zn}^{2+}>\mathrm{Pb}^{2+}> \\
\mathrm{Ni}^{2+}>\mathrm{Co}^{2+}>\mathrm{Cd}^{2+} \\
\end{array}$ & Bosso and Enzweiler (2002) \\
\hline $\begin{array}{l}\text { Turkish } \\
\text { 70\% Clinoptilolite }\end{array}$ & $\begin{array}{l}\mathrm{Co}^{2+} \\
\mathrm{Cu}^{2+} \\
\mathrm{Zn}^{2+} \\
\mathrm{Mn}^{2+}\end{array}$ & $\begin{array}{c}77.96 \%(0.448 \mathrm{meq} / \mathrm{g}) \\
66.10 \%(0.282 \mathrm{meq} / \mathrm{g}) \\
45.96 \%(0.268 \mathrm{meq} / \mathrm{g}) \\
19.84 \%(0.153 \mathrm{meq} / \mathrm{g}) \\
\mathrm{Co}^{2+}>\mathrm{Cu}^{2+}>\mathrm{Zn}^{2+}>\mathrm{Mn}^{2+}\end{array}$ & $\begin{array}{l}\text { Erdem et al. (2004) } \\
\text { Wang and Peng (2010) }\end{array}$ \\
\hline $\begin{array}{l}\text { Ukrainian } \\
\text { 75\% Clinoptilolite }\end{array}$ & $\begin{array}{l}\mathrm{Pb}^{2+} \\
\mathrm{Cu}^{2+} \\
\mathrm{Ni}^{2+} \\
\mathrm{Cd}^{2+}\end{array}$ & $\begin{array}{c}27.7 \mathrm{mg} / \mathrm{g} \\
25.76 \mathrm{mg} / \mathrm{g} \\
13.03 \mathrm{mg} / \mathrm{g} \\
4.22 \mathrm{mg} / \mathrm{g} \\
\mathrm{Pb}^{2+}>\mathrm{Cu}^{2+}>\mathrm{Cd}^{2}>\mathrm{Ni}^{2+}\end{array}$ & Sprynskyy et al. (2006) \\
\hline $\begin{array}{c}\text { Sardinian } \\
\text { 40-70\% Clinoptilolite }\end{array}$ & $\begin{array}{l}\mathrm{Pb}^{2+} \\
\mathrm{Cu}^{2+} \\
\mathrm{Zn}^{2+} \\
\mathrm{Cd}^{2+}\end{array}$ & $\begin{array}{c}0.34 \mathrm{meq} / \mathrm{g} \\
0.27-1.2 \mathrm{meq} / \mathrm{g} \\
0.1 \mathrm{meq} / \mathrm{g} \\
0.05-0.19 \mathrm{meq} / \mathrm{g} \\
\mathrm{Pb}^{2+}>\mathrm{Cu}^{2+}>\mathrm{Cd}^{2+} \cong \mathrm{Zn}^{2+}\end{array}$ & Cincotti et al. (2006) \\
\hline $\begin{array}{c}\text { Turkish Zeolite } \\
\text { (Clinoptilolite and Heulandite) }\end{array}$ & $\begin{array}{l}\mathrm{Pb}^{2+} \\
\mathrm{Zn}^{2+} \\
\mathrm{Cu}^{2+} \\
\mathrm{Ni}^{2+}\end{array}$ & $\begin{array}{cc}\text { Single- } & \text { Multi- } \\
0.730 \mathrm{meq} / \mathrm{g} & 0.299 \mathrm{meq} / \mathrm{g} \\
0.251 \mathrm{meq} / \mathrm{g} & 0.108 \mathrm{meq} / \mathrm{g} \\
0.227 \mathrm{meq} / \mathrm{g} & 0.022 \mathrm{meq} / \mathrm{g} \\
0.173 \mathrm{meq} / \mathrm{g} & 0.017 \mathrm{meq} / \mathrm{g} \\
\mathrm{Pb}^{2+}>\mathrm{Zn}^{2+}>\mathrm{Cu}^{2+}>\mathrm{Ni}^{2+} \\
\end{array}$ & $\begin{array}{l}\text { Oter and Akcay (2007) } \\
\text { Wang and Peng (2010) }\end{array}$ \\
\hline $\begin{array}{c}\text { Turkish } \\
\text { Clinoptilolite }\end{array}$ & $\begin{array}{l}\mathrm{Fe}^{3+} \\
\mathrm{Zn}^{2+} \\
\mathrm{Cu}^{2+} \\
\mathrm{Mn}^{2+}\end{array}$ & $\begin{array}{c}6.41 \mathrm{mg} / \mathrm{g} \\
1.60 \mathrm{mg} / \mathrm{g} \\
0.44 \mathrm{mg} / \mathrm{g} \\
0.37 \mathrm{mg} / \mathrm{g} \\
\mathrm{Fe}^{3+}>\mathrm{Zn}^{2+}>\mathrm{Cu}^{2+}>\mathrm{Mn}^{2+}\end{array}$ & Motsi et al. (2009) \\
\hline Brine Sediments & $\begin{array}{l}\mathrm{Zn}^{2+} \\
\mathrm{Cu}^{2+}\end{array}$ & $\begin{array}{r}4.85 \mathrm{mg} / \mathrm{g} \\
2.58 \mathrm{mg} / \mathrm{g} \\
\end{array}$ & \\
\hline Sawdust Materials & $\begin{array}{l}\mathrm{Zn}^{2+} \\
\mathrm{Cu}^{2+}\end{array}$ & $\begin{array}{l}4.69 \mathrm{mg} / \mathrm{g} \\
2.31 \mathrm{mg} / \mathrm{g}\end{array}$ & \\
\hline $\begin{array}{c}\text { Dried Marine Green } \\
\text { Macroalga Algal Biomass }\end{array}$ & $\begin{array}{l}\mathrm{Cu}^{2+} \\
\mathrm{Zn}^{2+}\end{array}$ & $\begin{array}{l}1.46 \mathrm{mmol} / \mathrm{g} \\
1.97 \mathrm{mmol} / \mathrm{g}\end{array}$ & Fu and Wang (2011) \\
\hline Rhizopus Oryzae Fungi Biomass & $\mathrm{Cu}^{2+}$ & $\begin{array}{c}19.4 \mathrm{mg} / \mathrm{g} \text { (Natural) } \\
43.7 \mathrm{mg} / \mathrm{g}(\mathrm{NaOH} \text {-treated) }\end{array}$ & \\
\hline
\end{tabular}




\subsubsection{Experimental Evaluation of Reaction Rate Kinetics}

The concurrent assessment of reaction rate constants and the orders of reaction with respect to all relevant entities under a variety of experimental conditions (i.e., concentrations) would require extensive experimentation and data analyses. Therefore, an abridged approach to evaluate one entity at a time is applied, by limiting the experimental conditions to assume that the reaction is irreversible and the concentrations of all but one entity remain approximately constant throughout the experiment. In this case, the reaction rate is assumed to follow a power law, approximated by the following expression in Equation 6.10 (Benjamin, 10, p. 97):

$$
\mathrm{r}_{\mathrm{A}}=-\mathrm{k}_{\mathrm{n}}[\mathrm{A}]^{\mathrm{n}}
$$

where [A] refers to the one reactant (i.e., HMI) whose concentration changes significantly during the experiment, such that the concentration is typically analyzed in a batch-mode reactor. To evaluate the experimental data, the integral method is a common approach in determining rate constants. Assuming that the reaction is the only process that alters $[\mathrm{A}]$, the rate of change of $[\mathrm{A}]$ is equated with the reaction rate as seen in Equation 6.11 (Benjamin, 10, p. 98):

$$
\mathrm{r}_{\mathrm{A}}=\frac{\mathrm{d}[\mathrm{A}]}{\mathrm{dt}} \rightarrow \int_{[\mathrm{A}]_{0}}^{[\mathrm{A}]_{\mathrm{t}}} \frac{\mathrm{d}[\mathrm{A}]}{\mathrm{r}_{\mathrm{A}}}=\int_{0}^{\mathrm{t}} \mathrm{dt}
$$

where $[\mathrm{A}]_{0}$ and $[\mathrm{A}]_{\mathrm{t}}$ refer to the initial reactant concentration and the reactant concentration at time $t$ (HMIs in meq/L), respectively. By predicting the order of the reaction $(n)$, the substitution for $r_{A}$ is made into Equation 6.11 to generate the integrated expression. The expressions for first-, second-, and $\mathrm{n}^{\text {th }}$ - order reactions are presented in Table 6.9.

Table 6.9 Rate Expressions for Reactant A Concentration in a Batch-Mode Reactor System

\begin{tabular}{|c|c|c|c|c|}
\hline \multirow{2}{*}{\multicolumn{2}{|c|}{$\begin{array}{c}\text { Rate } \\
\text { Expression }\end{array}$}} & \multicolumn{3}{|c|}{ Reaction Order (n) } \\
\hline & & 1 & 2 & $n^{\text {th }}$ \\
\hline \multicolumn{2}{|c|}{$\begin{array}{l}\text { Differential } \\
\text { Form }\end{array}$} & $\mathrm{r}_{\mathrm{A}}=\frac{\mathrm{d}[\mathrm{A}]}{\mathrm{dt}}=-\mathrm{k}_{\mathrm{n}=1}[\mathrm{~A}]$ & $\mathrm{r}_{\mathrm{A}}=\frac{\mathrm{d}[\mathrm{A}]}{\mathrm{dt}}=-\mathrm{k}_{\mathrm{n}=2}[\mathrm{~A}]^{2}$ & $\mathrm{r}_{\mathrm{A}}=\frac{\mathrm{d}[\mathrm{A}]}{\mathrm{dt}}=-\mathrm{k}_{\mathrm{n}}[\mathrm{A}]^{\mathrm{n}}$ \\
\hline \multicolumn{2}{|c|}{$\begin{array}{l}\text { Integral } \\
\text { Form }\end{array}$} & $\int \frac{\mathrm{d}[\mathrm{A}]}{[\mathrm{A}]}=-\mathrm{k}_{\mathrm{n}=1} \int \mathrm{dt}$ & $\int \frac{\mathrm{d}[\mathrm{A}]}{[\mathrm{A}]^{2}}=-\mathrm{k}_{\mathrm{n}=2} \int \mathrm{dt}$ & $\int \frac{\mathrm{dc}_{\mathrm{A}}}{\mathrm{c}_{\mathrm{A}}^{\mathrm{n}}}=-\mathrm{k}_{\mathrm{n}} \int \mathrm{dt}$ \\
\hline \multirow{2}{*}{$\begin{array}{l}\text { Integrated } \\
\text { Expression }\end{array}$} & $\mathrm{k}_{\mathrm{i}} \mathrm{t}$ & $\mathrm{k}_{\mathrm{n}=1} \mathrm{t}=\ln \left(\frac{[\mathrm{A}]_{0}}{[\mathrm{~A}]_{\mathrm{t}}}\right)$ & $\mathrm{k}_{\mathrm{n}=2} \mathrm{t}=\frac{1}{[\mathrm{~A}]_{\mathrm{t}}}-\frac{1}{[\mathrm{~A}]_{0}}$ & $\mathrm{k}_{\mathrm{n}} \mathrm{t}=\left(\frac{1}{\mathrm{n}-1}\right)\left([\mathrm{A}]_{\mathrm{t}}^{1-\mathrm{n}}-[\mathrm{A}]_{0}^{1-\mathrm{n}}\right)$ \\
\hline & {$[\mathrm{A}]_{\mathrm{t}}$} & {$[\mathrm{A}]_{\mathrm{t}}=[\mathrm{A}]_{0} \cdot\left(\mathrm{e}^{-\mathrm{k}_{\mathrm{n}=1} \mathrm{t}}\right)$} & {$[\mathrm{A}]_{\mathrm{t}}=\left(\frac{1}{[\mathrm{~A}]_{0}}+\mathrm{k}_{\mathrm{n}=2} \mathrm{t}\right)^{-}$} & {$[\mathrm{A}]_{\mathrm{t}}=\left([\mathrm{A}]_{0}^{1-\mathrm{n}}+(\mathrm{n}-1) \cdot \mathrm{k}_{\mathrm{n}} \mathrm{t}\right)^{\frac{1}{1-\mathrm{n}}}$} \\
\hline
\end{tabular}
(adapted from Benjamin, 2010, p. 100) 
By plotting $\ln \left([\mathrm{A}]_{\mathrm{t}}\right)$ versus $t$ for a first-order reaction (Chemistry LibreTexts, 2015), a slope of $-\mathrm{k}_{\mathrm{n}=1}\left(\right.$ in $\left.\min ^{-1}\right)\left(\right.$ Benjamin, 2010, p. 98) and y-intercept of $\ln \left([\mathrm{A}]_{0}\right)$ (Chemistry LibreTexts, 2015 ) is generated. By plotting $\frac{1}{[A]_{t}}$ versus $t$ for a second-order reaction, a slope of $k_{n=2}$ (in $\mathrm{L} /$ meq. min) and $\mathrm{y}$-intercept of $\frac{1}{[\mathrm{~A}]_{0}}$ (in $\mathrm{L} / \mathrm{meq}$ ) are generated (Benjamin, 2010, p. 100). If the reaction order prediction is correct, then the plot versus $\mathrm{t}$ would be linear with a strong correlation (CC value close to 1.0). However, an assumed rate expression will not always reproduce the experimental observations. If this is the case (i.e., a non-linear relationship is observed), then an alternative reaction order integer predication is made and the process is repeated. The integral method was applied to evaluate the experimental data of each HMI entity in this study, in each (single-, dual-, triple-, multi-) component system combination. The corresponding reaction rate data (reaction rate constants, predicated initial concentrations, and correlation coefficients) is presented in Table 6.10.

As reported in Benjamin (2010, p. 98), the first-order reaction rate plot of $\ln \left(\frac{[\mathrm{A}]_{t}}{[\mathrm{~A}]_{0}}\right)$ versus $t$ also generates a slope of $-\mathrm{k}_{\mathrm{n}=1}\left(\right.$ in $\left.\min ^{-1}\right)$. For each HMI entity in Table 6.10, only an average percent difference of $0.07 \%$ between the $\mathrm{k}_{\mathrm{n}=1}$ slope values is observed, with a stronger overall correlation $\left(\mathrm{CC}^{\mathrm{Y}}\right)$ in the $\ln \left([\mathrm{A}]_{\mathrm{t}}\right)$ versus $\mathrm{t}$ plot. The initial predication of a first-order reaction $(\mathrm{n}=1)$ generates a reasonable linear correlation, with $17.2 \%$ of the CC values below the acceptable range (0.85) and $41.1 \%$ above the excellent range (0.95-1.0). This prompted the decision to repeat the process and predict a second-order reaction $(n=2)$, which shows that $69.0 \%$ of the $\mathrm{CC}$ values are above the excellent range. With the HMI single-component system combinations as an example, Figure 6.6 illustrates the strong linear relationships of both reaction orders with the respective rate decay profiles (Benjamin, 2010, p. 99). In keeping with the zeolite selectivity order and regardless of which reaction order, the lead ion $\left(\mathrm{Pb}^{2+}\right)$ shows the strongest correlation and the nickel ion $\left(\mathrm{Ni}^{2+}\right)$ shows the weakest correlation. However, the predicated initial concentrations of $[\mathrm{A}]_{0}$ for the $\mathrm{Pb}^{2+}$ ion in the dual-, triple-, and multi-component system combinations at $\mathrm{n}=2$ are off-trend; such that the theoretically expected values of 5.0,3.33, and $2.0 \mathrm{meq} / \mathrm{L}$ for each component system, respectively, are exceeded by those concentrations predicated. Although a slightly stronger correlation is observed at $n=2$, it is proposed that the sorption reaction rate of the $\mathrm{Pb}^{2+}$ ion is best represented by the first-order reaction rate, such that 
the concentration is only dependent on the one reactant (a unimolecular reaction). This analysis thereby demonstrates that the rate of sorption changes for each HMI as additional HMIs are introduced into solution, under the testing conditions of the various component system combinations.

Table 6.10 Reaction Rate Data

\begin{tabular}{|c|c|c|c|c|c|c|c|c|}
\hline \multirow[b]{2}{*}{ SYSTEM } & \multicolumn{5}{|c|}{$\begin{array}{l}\text { First Order } \\
\quad n=1\end{array}$} & \multicolumn{3}{|c|}{$\begin{array}{c}\text { Second Order } \\
n=2\end{array}$} \\
\hline & $\begin{array}{c}-\mathrm{m}=\mathrm{k}_{\mathrm{n}=1} \\
\left(\mathrm{~min}^{-1}\right)\end{array}$ & $\mathbf{C C}^{\mathrm{X}}$ & $\begin{array}{c}-\mathrm{m}=\mathrm{k}_{\mathrm{n}=1} \\
\left(\min ^{-1}\right)\end{array}$ & $\begin{array}{c}{[\mathrm{A}]_{0}} \\
(\mathrm{meq} / \mathrm{L})\end{array}$ & $\mathbf{C C}^{\mathrm{Y}}$ & $\begin{array}{c}\mathrm{m}=\mathrm{k}_{\mathrm{n}=2} \\
(\mathrm{~L} / \mathrm{meq} \cdot \mathrm{min})\end{array}$ & $\begin{array}{c}\mathrm{A}]_{0} \\
(\mathrm{meq} / \mathrm{L})\end{array}$ & $\mathrm{CC}^{\mathrm{Z}}$ \\
\hline$[\mathrm{P}]$ & 0.0092 & 0.9358 & 0.0081 & 8.85 & 0.9719 & 0.0018 & 10.2 & 0.9969 \\
\hline [C] & 0.0016 & 0.7928 & 0.0012 & 9.63 & 0.9409 & 0.0001 & 9.64 & 0.9559 \\
\hline$[\mathrm{F}]$ & 0.0025 & 0.6235 & 0.0018 & 9.27 & 0.8785 & 0.0002 & 9.29 & 0.9080 \\
\hline$[\mathrm{N}]$ & 0.0008 & 0.0588 & 0.0005 & 9.63 & 0.7651 & 0.0001 & 9.62 & 0.7800 \\
\hline [Z] & 0.0014 & 0.8535 & 0.0012 & 9.72 & 0.9409 & 0.0001 & 9.74 & 0.9539 \\
\hline$[\mathrm{D}-\mathrm{PC}]-\mathrm{Pb}$ & 0.0134 & 0.9450 & 0.0119 & 4.24 & 0.9751 & 0.0080 & 7.26 & 0.9774 \\
\hline$[\mathrm{D}-\mathrm{PC}]-\mathrm{Cu}$ & 0.0009 & 0.7135 & 0.0007 & 4.88 & 0.9101 & 0.0001 & 4.88 & 0.9202 \\
\hline$[\mathrm{D}-\mathrm{PF}]-\mathrm{Pb}$ & 0.0099 & 0.9548 & 0.0089 & 4.49 & 0.9783 & 0.0042 & 5.47 & 0.9940 \\
\hline$[\mathrm{D}-\mathrm{PF}]-\mathrm{Fe}$ & 0.0032 & 0.7322 & 0.0024 & 4.59 & 0.9218 & 0.0006 & 4.61 & 0.9544 \\
\hline$[\mathrm{D}-\mathrm{PN}]-\mathrm{Pb}$ & 0.0139 & 0.9459 & 0.0123 & 4.21 & 0.9762 & 0.0087 & 7.89 & 0.9758 \\
\hline [D-PN]-Ni & 0.0006 & 0.1422 & 0.0004 & 4.88 & 0.5693 & 0.0001 & 4.88 & 0.5728 \\
\hline$[\mathrm{D}-\mathrm{PZ}]-\mathrm{Pb}$ & 0.0136 & 0.9383 & 0.0121 & 4.21 & 0.9704 & 0.0083 & 7.19 & 0.9831 \\
\hline [D-PZ]-Zn & 0.0008 & 0.8591 & 0.0007 & 4.94 & 0.9156 & 0.0001 & 4.94 & 0.9231 \\
\hline$[\mathrm{D}-\mathrm{CF}]-\mathrm{Cu}$ & 0.0016 & 0.9130 & 0.0014 & 4.89 & 0.9527 & 0.0003 & 4.90 & 0.9664 \\
\hline$[\mathrm{D}-\mathrm{CF}]-\mathrm{Fe}$ & 0.0038 & 0.6898 & 0.0028 & 4.49 & 0.9097 & 0.0008 & 4.52 & 0.9506 \\
\hline$[\mathrm{D}-\mathrm{CN}]-\mathrm{Cu}$ & 0.0023 & 0.8368 & 0.0018 & 4.76 & 0.9438 & 0.0004 & 4.78 & 0.9623 \\
\hline$[\mathrm{D}-\mathrm{CN}]-\mathrm{Ni}$ & 0.0008 & 0.1051 & 0.0005 & 4.82 & 0.7680 & 0.0001 & 4.82 & 0.7789 \\
\hline$[\mathrm{D}-\mathrm{FZ}]-\mathrm{Fe}$ & 0.0040 & 0.6208 & 0.0029 & 4.41 & 0.8970 & 0.0008 & 4.44 & 0.9424 \\
\hline$[\mathrm{D}-\mathrm{FZ}]-\mathrm{Zn}$ & 0.0013 & 0.9114 & 0.0011 & 4.90 & 0.9678 & 0.0002 & 4.90 & 0.9756 \\
\hline [D-NZ]-Ni & 0.0005 & -0.7590 & 0.0002 & 4.85 & 0.4677 & 0.0001 & 4.85 & 0.4720 \\
\hline [D-NZ]-Zn & 0.0014 & 0.9171 & 0.0012 & 4.91 & 0.9519 & 0.0003 & 4.92 & 0.9628 \\
\hline$[\mathrm{T}]-\mathrm{Pb}$ & 0.0146 & 0.9395 & 0.0129 & 2.76 & 0.9737 & 0.0146 & 5.77 & 0.9733 \\
\hline$[\mathrm{T}]-\mathrm{Cu}$ & 0.0014 & 0.8438 & 0.0011 & 3.24 & 0.9413 & 0.0004 & 3.24 & 0.9528 \\
\hline$[\mathrm{T}]-\mathrm{Fe}$ & 0.0046 & 0.7071 & 0.0035 & 2.93 & 0.9132 & 0.0015 & 2.96 & 0.9601 \\
\hline$[\mathrm{M}]-\mathrm{Pb}$ & 0.0183 & 0.9073 & 0.0158 & 1.51 & 0.9553 & 0.0422 & 12.5 & 0.9760 \\
\hline$[\mathrm{M}]-\mathrm{Cu}$ & 0.0016 & 0.9192 & 0.0014 & 1.96 & 0.9590 & 0.0008 & 1.96 & 0.9700 \\
\hline$[\mathrm{M}]-\mathrm{Fe}$ & 0.0056 & 0.7110 & 0.0042 & 1.71 & 0.9156 & 0.0034 & 1.74 & 0.9695 \\
\hline$[\mathrm{M}]-\mathrm{Ni}$ & 0.0007 & 0.3354 & 0.0005 & 1.94 & 0.7790 & 0.0003 & 1.94 & 0.7869 \\
\hline$[\mathrm{M}]-\mathrm{Zn}$ & 0.0011 & 0.9694 & 0.0011 & 2.00 & 0.9695 & 0.0006 & 2.00 & 0.9752 \\
\hline
\end{tabular}




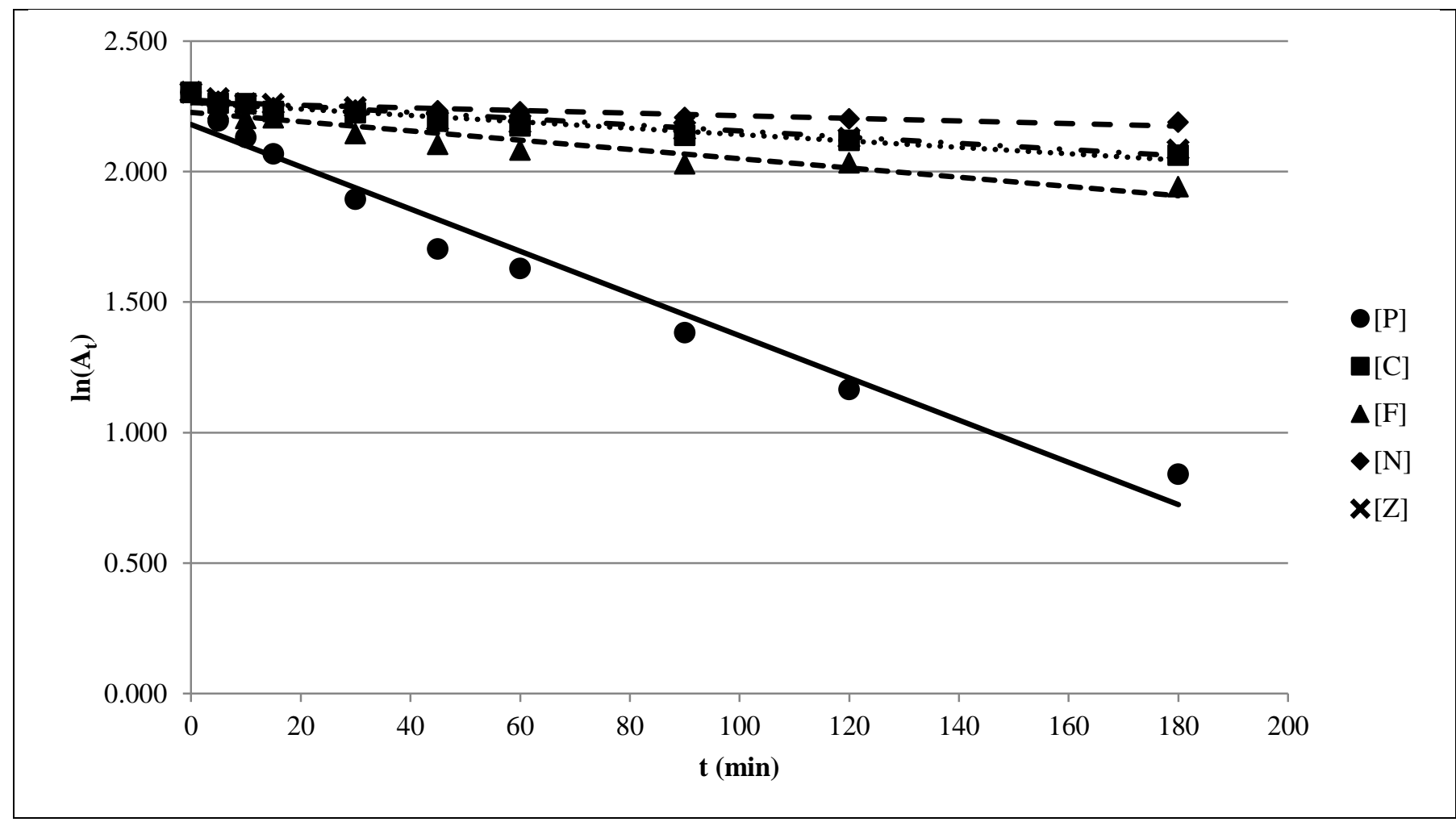

a.

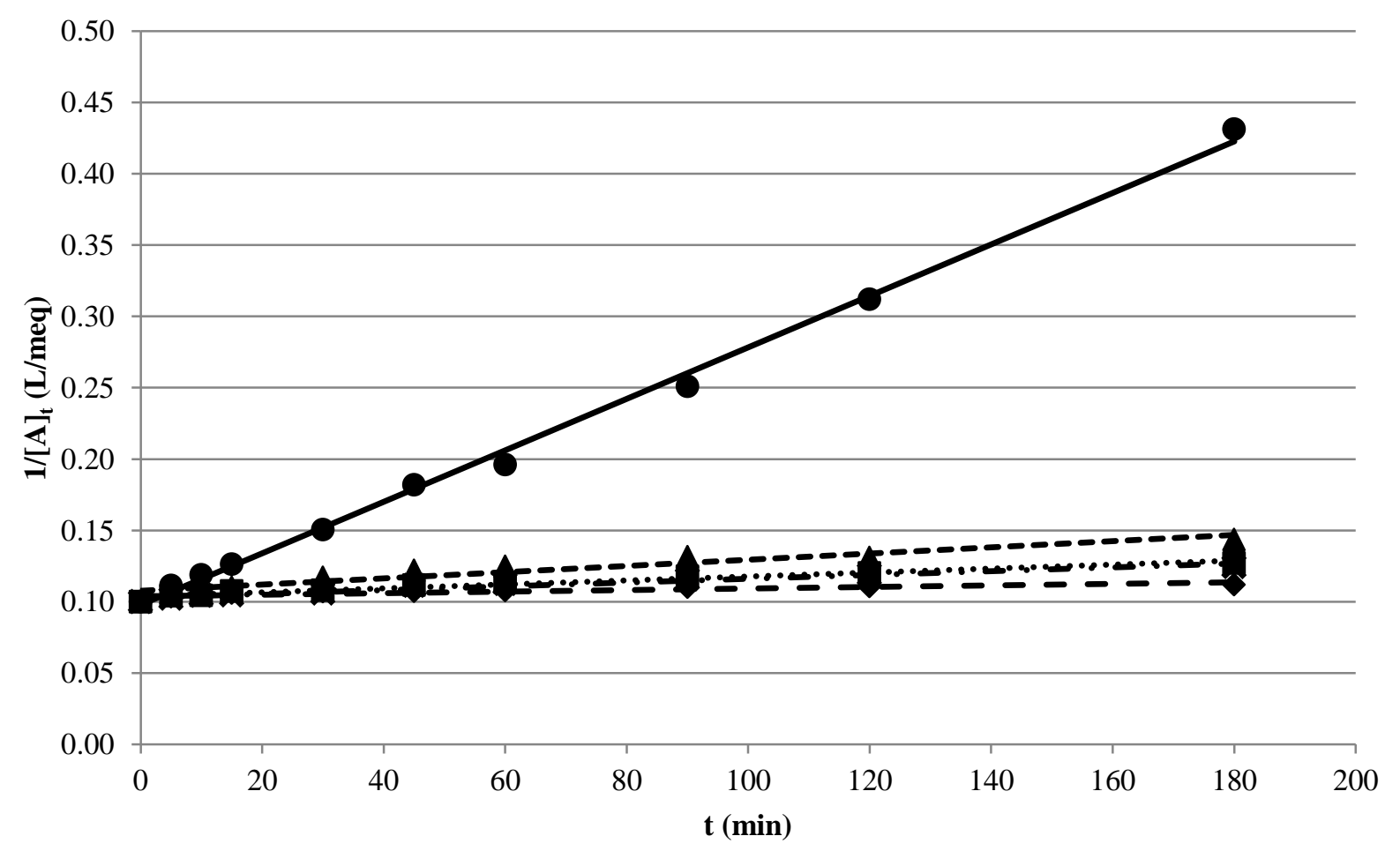

Figure 6.6 Single-Component System Reaction Rate Decay Profiles

a. First-Order; b. Second-Order 


\subsubsection{Sensitivity Analysis Considerations of Model Parameters}

In the assessment of the sensitivity of the experimental parameters in each non-linear form of a kinetic model, it is observed that the amount of HMI sorbed at equilibrium $\left(\mathrm{q}_{\mathrm{e}} ; \mathrm{meq} / \mathrm{g}\right)$ and the rate constant $\left(\mathrm{k}_{2} ; \mathrm{g} / \mathrm{meq} \cdot \mathrm{min}\right)$ for the pseudo-second-order (PSO) model are critical variables; consistent with the findings presented by Dridi-Dhaouadi et al. (2011). The research conducted by Dridi-Dhaouadi et al. (2011) investigates the sorption of inorganic (heavy metal; $\mathrm{Pb}^{2+}$ ) and organic pollutants by biomass, in both single- and dual-component systems; demonstrating a strong correlation with the PSO model, the analysis indicates that the $\mathrm{q}_{\mathrm{e}}$ uptake is more sensitive than the $\mathrm{k}_{2}$ constant. The study presented in the Dissertation document demonstrated that the zeolite mineral exhibits the greatest preference towards the lead ion $\left(\mathrm{Pb}^{2+}\right)$; considered in its single-component system $[\mathrm{P}]$, with reference to Table 6.4 and Figure 6.2a of this chapter, a strong correlation of 0.9926 is observed based on the plot of $t / q_{t}$ versus $t$ under the specified testing conditions. As discussed in the previous subsection in the evaluation of the experimental data, the lead ion exhibits a different reaction rate compared to the other HMIs when combined in the various component systems. Accordingly, the quality control protocol discussed in Section 3.3. prompts the need to allocate thoughtful attention to the influent concentration parameter when a kinetic model and reaction rate assessment are conducted, in order to predict the sorption of the $\mathrm{Pb}^{2+}$ ion by natural zeolite.

It is important to note that the process variables of the zeolite source and particle size, sorbent-sorbate dosage, initial concentration, $\mathrm{pH}$ level, set-temperature as well as agitation speed are kept constant in this study. This was maintained in order to create a purposeful experimental design, with feasibility, to best observe the impact of the heavy metallic ions in combination. Although the pseudo-second-order kinetic model has demonstrated the strongest representation of the data in uptake over time, to declare the reaction based mechanism of chemisorption as the overall rate-determining step is not the objective of this study. Accordingly, it is recommended that the variation of the process variables would be required to make that final statement. Rather, this study has investigated simultaneous sorption of the HMIs, with the quantification of uptake and rate of interference of these HMIs in numerous component system combinations. The selected reaction and diffusion kinetic models demonstrated in this study have provided a stronger understanding into the associated mechanisms prior to equilibrium of the overall sorption process. 


\subsection{Conclusions}

The sorption kinetics of the multiple heavy metallic ions (HMIs) predominantly found in mine wastewater has been investigated. With the systematic experimental design of five HMIs and zeolite in its natural state, the sorption uptake rate and subsequent interference of these HMIs in combination has been quantified and modelled. Both the pseudo-second-order (PSO) and intra-particle diffusion kinetic model demonstrate a very strong correlation of the extensive experimental data. However, the PSO model demonstrated a stronger performance, and further contributed to the assessment of the zeolite mineral performance and the fundamental mechanisms associated with the sorption process. Usually, the equilibrium sorption capacity is unknown. The process of chemisorption is significantly slow, and the sorbed amount is smaller than that at equilibrium. Study limitations include the fact that the time required to reach equilibrium is very long in various sorption systems. Although rapid uptake was observed in the first 45 minutes, the desired balanced state is achieved beyond the analysis period of kinetic analysis. A significant improvement to this study would be to extend the 3-hour contact time, in order to associate the equilibrium state capacity experimentally and theoretically, as well as evaluate various operative conditions to generate further insight into associated mechanisms. Nevertheless, the PSO model provides important information of the sorption capacity, initial sorption rate and overall rate coefficients devoid of any parameter in advance (Ho and Ofomaja, 2006).

\subsection{Future Works}

Further investigations in the variation of operation parameters are needed from this phase to develop a greater understanding of the kinetic reaction relationships. Through modeled kinetics, the prediction of sorption rate is significant for batch system design, in order to determine the solute uptake required for optimal conditions of full-scale processes. To be presented in Chapter 7, future work on this research project focuses on the interaction of heavy metallic ions combined in the multi-component system using natural zeolite in continuous-mode, with the design of a dual-column, fixed bed reactor, treatment system prototype. 


\title{
7. DUAL-COLUMN SORPTION SYSTEM PERFORMANCE
}

\author{
An Innovative Dual-Column System for \\ Heavy Metallic Ion Sorption by Natural Zeolite \\ Applied Sciences - Special Issue \\ Wastewater Treatment and Reuse Technologies \\ 2017. 7(8). 795. doi:10.3390/app7080795.
}

Fundamental components of this chapter were also presented as a paper at the WEAO2018 Technical Symposium ('Industrial Treatment B' Session); referenced in Appendix E of the Dissertation document.

\begin{abstract}
This study investigates the design and performance of a novel sorption system containing natural zeolite. The apparatus consists of packed, fixed-bed, dual-columns with custom automated controls and sampling chambers, connected in series and stock fed by a metering pump at a controlled adjustable distribution. The purpose of the system is to remove heavy metallic ions commonly found in industrial wastewater effluent, including lead $\left(\mathrm{Pb}^{2+}\right)$, copper $\left(\mathrm{Cu}^{2+}\right)$, iron $\left(\mathrm{Fe}^{3+}\right)$, nickel $\left(\mathrm{Ni}^{2+}\right)$ and zinc $\left(\mathrm{Zn}^{2+}\right)$, combined in equal equivalence to form an acidified total $10 \mathrm{meq} / \mathrm{L}$ aqueous solution. Reported trends on the zeolite's preference to these heavy metallic ions is established in the system breakthrough curve, as $\mathrm{Pb}^{2+}>\mathrm{Fe}^{3+}>\mathrm{Cu}^{2+}>$ $\mathrm{Zn}^{2+}>\mathrm{Ni}^{2+}$. Within a 3-hour contact period, $\mathrm{Pb}^{2+}$ is completely removed from both columns. Insufficient $\mathrm{Ni}^{2+}$ removal is achieved by either column with the promptest breakthrough attained, as zeolite demonstrates the least affinity towards it; however, a $49.0 \%$ removal is observed in the cumulative collection at the completion of the analysis period. The empty bed contact times for the first and second columns are 20 and 30 minutes, respectively; indicating a higher bed capacity at breakthrough and a lower usage rate of the zeolite mineral in the second column. This sorption system experimentally demonstrates the potential for industrial wastewater treatment technology development.
\end{abstract}




\subsection{Introduction}

The performance of columns or fixed-bed reactors (FBR) is convenient for industrial scale applications (Vukojevic Medvidovic et al., 2013), which requires less investment and operational costs, and is more economically feasible than its discontinuous batch-mode counterparts (Nuic et al., 2016). FBR columns have demonstrated performance efficiency in treating large volumes, and are frequently implemented in sorption studies. Operations are affected by equilibrium (isotherm and capacity), kinetic (diffusion and convection coefficients) and hydraulic (liquid holdup, geometric analogies and mal-distribution) factors (Inglezakis, 2010a). In practice, the influence of operative conditions on the overall system performance is not fully experimentally verified (Curkovic et al., 1997; Erdol Aydin and Nasun Saygili, 2009; Inglezakis et al., 2001a), but are extremely important to large-scale development. Although the FBR system is highly valuable, its analysis is unpredictably multi-faceted (Inglezakis, 2010a; 2010b) and even more so with the presence of numerous interfering ions. Complications due to ion competition and solutesurface interactions (Mohan and Chander, 2006), as well as the unique affinity sequences and sorbent material selectivity (Nuic et al., 2015), have been reported.

The first three phases of this research project (Chapters 4 to 6)were conducted in batchmode, which reveal a key trend among the HMIs selected as lead $\left(\mathrm{Pb}^{2+}\right)>>$ iron $\left(\mathrm{Fe}^{3+}\right)>$ copper $\left(\mathrm{Cu}^{2+}\right)>\operatorname{zinc}\left(\mathrm{Zn}^{2+}\right)>$ nickel $\left(\mathrm{Ni}^{2+}\right)$. The findings of these preliminary phases have established a platform for the design of the sorption system in continuous mode, presented in this chapter.

Existing column experimental designs involve various limitations, including:

1. The evaluation of predominantly single- or dual-component HMI system combinations;

2. The implementation of primarily slender column aspect ratios (i.e., bed depth/particle diameter, column height/diameter), causing a challenge to eventual scale-up design;

3. The use of inconsistent and/or vague sorbent compaction techniques, and;

4. The application of simple, idealized flow patterns (i.e., set single and continuous flow rate).

The objective of this final research phase is to develop a novel dual-column sorption system to overcome some of these shortcomings. Important design factors such as the zeolite compaction, column dimensions and aspect ratios, flow control, sampling and analytical procedure, will be taken into consideration. The exclusivity of this prototype is attributed to an 
automated, variable-flow configuration with a custom sampling technique. In contrast to most previous single-component sorption set-ups, this study evaluates the simultaneous sorption process by natural zeolite of five commonly occurring HMIs found in industrial wastewater effluent. This study will demonstrate the effectiveness and the removal efficiency in a continuous-flow FBR system over a 3-hour contact duration from the dual columns, providing insights on HMIs selectivity and treatment system breakthroughs. It is envisaged that this research will provide much-needed information to the wastewater treatment industry for the design and implementation of innovative sorption technologies.

\subsection{Materials and Methods}

Refer to Chapter 3 for the consolidated experimental methodology; sorbent material, sorbate solution, and analytical procedure. All analyses are conducted in continuous-mode, creating the synthetic simple solute HMI solution in the multi-[M] component system combination. The following subsections discuss the associated breakthrough and exhaustion principles as well as the treatment system prototype design details for this study.

\subsubsection{Packed Fixed-Bed Column Design Considerations}

When the concentration of the effluent reaches $5-10 \%$ of the influent, this point on a typical S-shaped breakthrough curve is commonly referred to as the 'breakthrough point' or 'breakpoint' (BP) (Beyazit, 2013). The point of column exhaustion (EP) is when the effluent reaches maximum capacity to $90-95 \%$ of its influent concentration value (Nuic et al., 2013; Vukojevic Medvidovic et al., 2006). The efficiency of the column performance is related to the bed capacity at breakthrough and at exhaustion, represented by the following relationship (Nuic et al., 2013; Vukojevic Medvidovic et al., 2006):

$$
\eta=\frac{C_{B P}}{C_{E P}}
$$

where $\eta$ is the column efficiency (degree of saturation), $C_{B P}$ is the breakthrough capacity of the bed (in meq/g), and $\mathrm{C}_{\mathrm{EP}}$ is the maximum capacity at exhaustion of the bed, defined by the total amount of HMI ions bound in the zeolite (or exchanged in the packed fixed-bed) (in meq/g). 
The breakthrough capacity and equilibrium capacity are further expressed in Equation 7.2 and Equation 7.3, respectively (Nuic et al., 2013; Vukojevic Medvidovic et al., 2006):

$$
\begin{gathered}
C_{\mathrm{BP}}=\frac{\int_{0}^{V_{\mathrm{BP}}}\left(C_{0}-C_{t}\right) d V}{\rho H A}=\frac{C_{O} \cdot V_{B P}}{m}=\frac{\eta_{B P}}{m_{Z}} \\
C_{E P}=\frac{\int_{0}^{V_{\mathrm{EP}}}\left(C_{0}-C_{t}\right) d V}{\rho H A}=\frac{\int_{0}^{V_{E P}}\left(C_{O}-C_{t}\right) d V}{m}=\frac{\eta_{E P}}{m_{Z}}
\end{gathered}
$$

where $\mathrm{V}_{\mathrm{BP}}$ is the effluent volume collected up to breakthrough point (BP) (in $\mathrm{L}$ ), $\mathrm{V}_{\mathrm{EP}}$ is the effluent volume at which the exhaustion point (EP) is reached in the zeolite bed (in $\mathrm{L}$ ), $\mathrm{C}_{\mathrm{O}}$ is the influent concentration (in meq/L), $C_{t}$ is the effluent concentration (in meq/L), $\rho$ is the packing density of the bed (in $\mathrm{g} / \mathrm{cm}^{3}$ ), $\mathrm{H}$ is the bed depth (in $\mathrm{cm}$ ), $\mathrm{A}$ is the bed cross-sectional area (in $\mathrm{cm}^{2}$ ), $\mathrm{m}_{\mathrm{Z}}$ is the zeolite mass (in $\mathrm{g}$ ); where $\eta_{\mathrm{BP}}$ and $\eta_{\mathrm{EP}}$ is the total amount of HMI ions removed up to $\mathrm{BP}$ and $\mathrm{EP}$ (in meq), respectively.

The empty bed contact time (EBCT) is a primary design variable (Droste, 1997, p. 489), which represents the length of time a liquid stream is in contact with the bed without sorbent. It is related to the removal kinetics of the column treatment system medium (Reed et al., 1996; Jarvie et al., 2005). This is represented by the following relationship between the bed depth (H) in the column and the feed solution velocity (v) (Droste, 1997, p. 478; Nuic et al., 2015; Vukojevic Medvidovic et al., 2006):

$$
\text { EBCT }=\frac{H}{v}=\frac{H}{(Q / A)}=\frac{d^{2} \pi H}{4 Q}
$$

Research conducted by Vukojevic Medvidovic et al. (2006) demonstrates that the breakthrough curve results reveal that the flow through the column determines the EBCT; with the same initial concentration, the increase in flow rate decreases the contact time and increases the mass transfer zone (MTZ) height. The MTZ is the restricted area where the exchange process occurs, and is defined as the zeolite layer height between the equilibrium zone and the unused bed zone (Vukojevic Medvidovic et al., 2006); where the effluent concentration varies from 595\% of the influent concentration (Nuic et al., 2015). As the HMI solution is fed through the packed fixed-bed, the MTZ moves in the direction of flow and eventually reaches the exit (Nuic et al., 2013; Vukojevic Medvidovic et al., 2006). Peric et al. (2009) distinctly demonstrates the 
importance of the column bed depth on the removal of lead from aqueous solutions. The results show that as the bed depth increases, a delay in breakthrough and exhaustion occurs, with an increase of the MTZ height. The higher the bed depth, the longer the service time at various breakthrough points due to the increase in binding sites on the sorbent material (zeolite mineral) (Han et al., 2006). Adequate wetting of the zeolite, and ideal contact time between the zeolite and solution interface are important for mass transfer and equilibrium conditions based on the selection of the flow rate and particle size. To minimize possible wall and axial dispersion effects in the fixed-bed column, the bed depth-to-particle diameter ratio $\left(\mathrm{H} / \mathrm{d}_{\mathrm{p}}\right)$ must be greater than 20 . At a higher $\mathrm{H} / \mathrm{d}_{\mathrm{p}}$ ratio, the breakthrough point appears later and the curve is steeper.

The usage rate $\left(v_{U}\right.$, in $\left.g / L\right)$ determines the rate at which the sorbent would be exhausted and how often it must be replaced or regenerated, and is expressed in the following relationship (Jarvie et al., 2005; Mohan and Chander, 2006; Nuic et al., 2015):

$$
\mathrm{v}_{\mathrm{U}}=\frac{\mathrm{m}_{\mathrm{Z}}}{\mathrm{V}_{\mathrm{BP}}}
$$

where $m_{Z}$ is the zeolite mass in the bed (in $g$ ) and $V_{B P}$ is the volume of the effluent treated at breakthrough (BP) (in L) (Nuic et al., 2015). Inglezakis (2010a) states that it is extremely difficult to model multi-component system interactions, as numerous time-consuming data are required and the process involves significant mathematical complexity. Breakthrough and exhaustion thresholds of specific HMIs within a fixed-bed are important for experimental specific conditions. In order to optimize the liquid-solid contact time and removal capacity, it is necessary to develop a greater understanding of these relationships, between EBCT and usage rate (Inglezakis, 2010a). 


\subsubsection{Sorption Treatment System Design}

Based on qualitative observations, the uptake of counter-ions in a continuous column system is favoured by various factors, including: a strong preference of the zeolite for the HMI counterions in solution, low concentration of HMI counter-ions, small and uniform particle size, high volume capacity and low degree of cross-linking, elevated temperature and low flow rate, as well as a high column height or aspect ratio (Helfferich, 1962, p. 427). The apparatus development considered an extensive material and equipment selection process, with numerous stages of optimization in order to maintain flow continuity and repeatability. The prototype development is highlighted in Appendix A.10 of the Dissertation document.

The final design was adopted in consideration of the following factors:

- Zeolite Compaction Technique

- Regulated Layers of Dry Mass

- Systematic Tampered Compaction

- Column Dimensions

- Modular Design

- Internal Diameter (1 in)

- Sorption Column Height (1 ft)

- Flow Configuration

- Upflow Distribution

- Dual-Column Series Connection

- Methodical Flow Rate Variability
- Pump Type

- Diaphragm Metering

- Sampling Method

- Automated Mode Controls

- Customized Sampling Chambers

- Modes' Interchange in Five (5)-minute Intervals

- Analysis Period

○ Three (3)-hour Contact Period

Based on these critical parameters, the sorption system design was finalized. Figure 7.1 is a schematic representation of the constructed prototype, detailing the flow paths through the system. The fundamental components include:

- HMI Multi-Component Influent Stock

- Metering Pump

- Silicon Tubing and Polyvinyl chloride (PVC) Connections

- Check Valves

- Automatable Solenoid Valves (symbol S)
- Packed Fixed-Bed Sorption Columns

- Custom Sampling Chambers

- Sampling Ports

- Effluent Collection Basin 


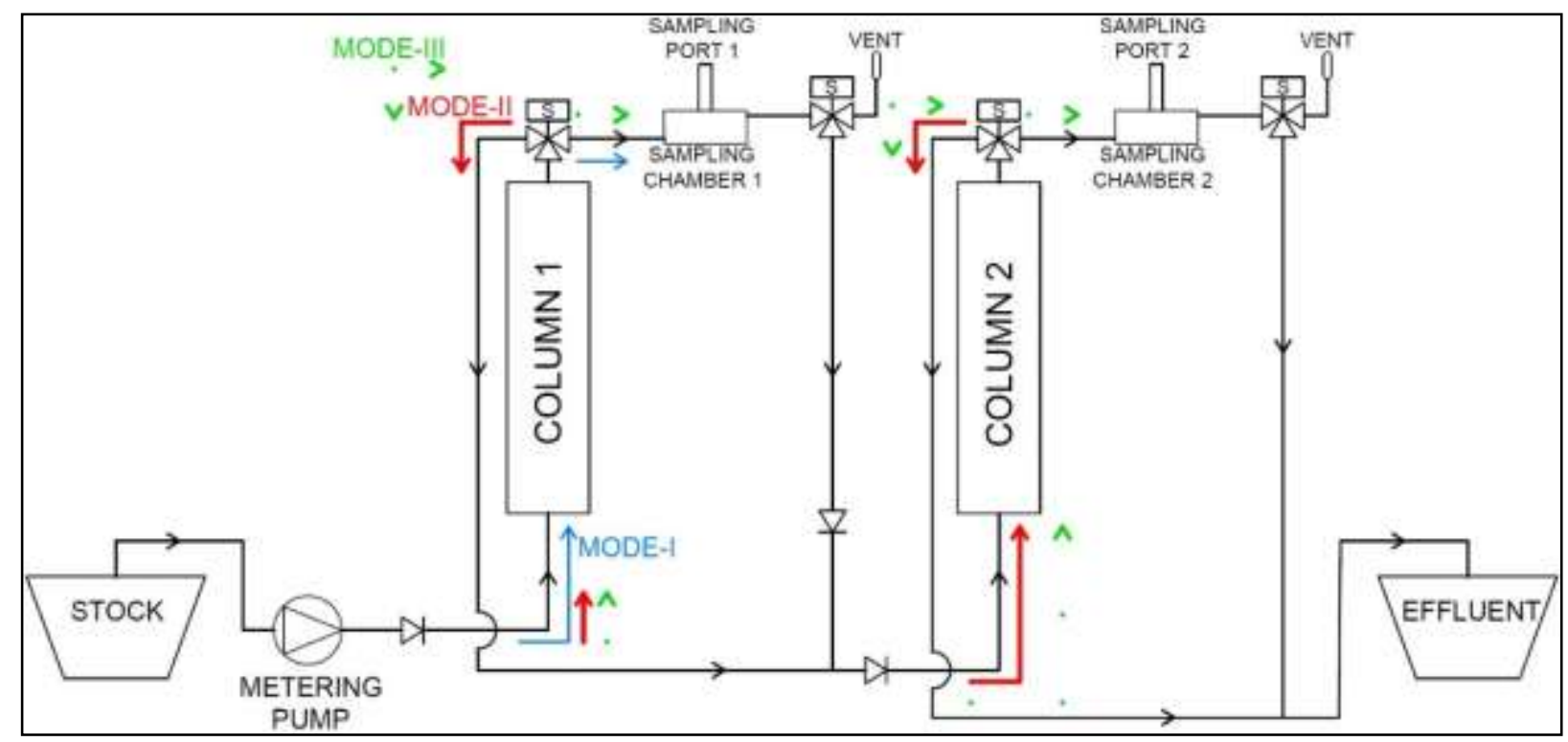

Figure 7.1 Schematic of Automated Sorption System Prototype Flow Path Layout (adapted from Ciosek and Luk, 2017d)

\subsubsection{Column Dimensions}

The column was made of a circular section of clear PVC SCH-40 pipe (Part No. r4-1000; Fabco Plastics; Maple, ONT, Canada), $30.48 \mathrm{~cm}$ in height with $2.61 \mathrm{~cm}$ internal diameter. In order to minimize potential effects of wall and axial dispersion in the columns, the bed depth-toparticle diameter ratio should be kept greater than 20 (Peric et al., 2009). Using the average nominal zeolite diameter of $1.00 \mathrm{~mm}$ as a reference, this ratio translated to over 300 for the design. The cleaned and dry zeolite particles were added to the column at nine layers applied at 20-mL or $16.9 \mathrm{~g}$ amounts. Each layer was compacted with medium force, pounding six times with a customized PVC plunger of a diameter equal to the internal diameter of the column; such that the column height and the zeolite bed depth are equivalent. Inert plastic mesh with a smaller size than the minimum zeolite particle gradation of $0.841 \mathrm{~mm}$ was used to contain the zeolite material, and permitted sample flow through the columns. This mesh was set at each end of the column, within the two halves of a PVC SCH-80 socket union fitting incorporating a viton o-ring (Part No. 897010; Fabco Plastics; Maple, ONT, Canada), connected to a nominal $1 \times 1 / 2$-inch PVC SCH-40 reducer bushing (Part No. 438130 (slip $\times$ FPT); Fabco Plastics; Maple, ONT, Canada). All components were connected to 1/4-inch silicon tubing and with corresponding adapters and nipples fittings. 


\subsubsection{2. $\quad$ Flow Rate and Configuration}

As a dual-stage system, the two columns were connected in a serial-flow arrangement, such that the first column received the original stock with a higher HMI concentration and the second column received the effluent from the first column. The upflow configuration ensures an overall better quality of flow, with a low liquid hold-up and a good stock feed distribution across the column cross sectional area (Inglezakis, 2010a); enabling the media to move upward and fall away separately from the filter, swirling the solution within the system to yield a longer contact time (Andoh, 2014). In contrast, for the downflow mode, an increase in pressure drop and flooding of the column bed is more probable (Inglezakis, 2010a). Consequently, the stock was fed in an upflow direction to ensure proper and thorough distribution to the column beds and to minimize the need for backwashing (Gregory, 2010) and head loss effects.

A critical parameter in the design process is the flow rate. Existing research demonstrates that lower flow rates result in high detention times in the column, which is needed due to the relatively slow uptake rate of zeolite (Inglezakis et al., 2002; 2004). The HMI solution volume element is in contact with a given zeolite bed layer for only a limited period of time. Consequently, equilibrium is not usually achieved and results in a lower overall uptake of HMIs from the influent stock solution. Preliminary testing involved a peristaltic pump, using the corresponding silicon rubber tubing. Significant back pressure was observed and the capacity of the peristaltic pump was hindered. Consequently, the required flow rate was unachievable; the rotational speed and strength decreased for the feed to completely traverse through the entire system. Subsequently, a diaphragm-type metering pump (No. 950218125-C Plus, max 45-LPD, 80-psi, 125-AC, 50/60-Hz; PULSAtron; Punta Gorda, FL, USA) was employed in the final design, which mechanically facilitates the desired stock feeding rate. Based on the 45-LPD $(31.25 \mathrm{~mL} / \mathrm{min})$ capacity of the metering pump, preliminary flow rate testing of the pump set to $100 \%$ stroke (mechanically pumped volume) and 50\% rate established an initial, repeatable, point-of-reference flow rate of $6.36 \pm 0.32 \mathrm{~mL} / \mathrm{min}$. This stroke-rate setting was maintained and is comparable to the lower end of the $6-18 \mathrm{~mL} / \mathrm{min}$ range recommended by Inglezakis et al. (2002; 2004), to provide sufficient detention time in the system. 


\subsubsection{Sampling Method}

Another critical component to the design is the sampling method, and how to maintain continuous flow through the system while sampling the effluent of both columns. Due to the relatively slow feeding rate selected for this study, the time to collect the desired sample volume for dilution and ICP-AES analysis would require residual sample volume and minutes of valuable contact time. Therefore, three-way solenoid valves (No. 00457979, 0124-C, 1/8-FKMPP, NPT-1/4, max 145-psi, 24-V, 60-Hz, 8-W, 38-mL; burkert; Ingelfingen, Germany) were implemented as a device to ensure that while a sample volume is collected at the desired sampling time, both columns would still be fed continuously by unique the modes of operation. The MODE valves and custom fabricated rotating 30-mL sampling chambers were attached to the top exit of each sorption column, with accessible sampling ports. A second three-way solenoid VENT valve was included at the exit of each sampling chamber to introduce an air vent to assist in rapid sample extraction by preventing vacuum pockets within the sample chamber and discharge tubing. A multi-turn valve was included at the exit of the vent for the first column to introduce minor back pressure similar to that of the second column, so as to not significantly alter the flow characteristics through the first column. Check valves were placed at critical locations throughout the hydraulic circuit to prevent back flows. 


\subsubsection{System Modes of Operation}

The sorption system presented in this paper is comprised of three distinct modes of operation that are controlled by the MODE valves for each column, in the following sequence:

- MODE-I

○ Activate System

- Prime Inlet Tubing and Check Valve

- Fill Inlet Connection Cavity

○ Fill Sorption Column 1 (8:39 min:s to fill)

o Fill Sample Chamber 1 (3:40 min:s to fill)

- MODE-II

○ Divert Flow from Sampling Chambers

- Activate VENT Valve

- Collect Column 1 Sample (C1-A at 18:50 min:s)

○ 'Circulate' Flow through Sorption Columns

○ Fill Sorption Column 2 (8:27 min:s to fill)

- MODE-III

○ 'Pulse' Flow between Sampling Chambers

- Divert 100\% flow, 50\% time per Timer Setting

○ Fill Sampling Chambers

- MODE-II

○ Detour Flow from Sampling Chambers

- Activate VENT Valve

- Collect Samples from Sorption Columns

(C1-B and C2-B at 42:50 min:s)

○ 'Circulate' Flow through Sorption Columns

- >INITIATE MODE-II/III SEQUENCE<

Figure 7.2 presents the arrangement of the prototype components, including an adjustable bi-stable timer which determines the time division modulation of the MODE and VENT solenoid valves.

Activating the process in Mode-I, the fluid element was mechanically pumped from the acidified 3-L multi-component influent stock. Once the pump is turned on, the inlet tubing was primed with the influent stock and passed the column check valve at the system inlet. The fluid element passed through the mesh-union fitting and reached the base of the first column (C1), and traversed up through the sample chamber entry solenoid valve to the first sampling chamber. Once the 30-mL sample chamber was filled, the fluid element began to drip at its exit against the multi-turn valve, which was an indication to switch the sample chamber entry solenoid MODE valves to Mode-II using the automated mode controls. 
In Mode-II, the fluid element by-passes the first sampling chamber (SC1), continued to traverse through column 1 (C1) and began to fill column 2 (C2). The fluid element did not crosscircuit back towards the exits of first column, due to the connection of additional check valves connection located at the entry of the second column. While the fluid element traversed up both columns $\mathrm{C} 1$ and $\mathrm{C} 2$, the sampling chamber exit solenoid VENT valve was switched from closed to open. The sampling port tube was uncapped, twisted using the custom rotating handle and inverted to draw a 30-mL sample. The VENT valve was then turned off (closed from atmosphere). It is important to note that the inlet-outlet offset of the sampling chambers guarantees a highly repeatable sample volume. It is designed to minimize cross-contamination, for when the chamber is rotated from the vertical upward (sample collection in Mode-III) to downward (sample dispense in Mode-II and VENT) position, the chamber contents are completely void.

Once C2 was filled, both MODE valves of the sampling chambers were switched from Mode-II to Mode-III. The fluid element now simultaneously traverses through $\mathrm{C} 1$ and $\mathrm{C} 2$, while filling $\mathrm{SC} 1$ and $\mathrm{SC} 2$, dividing the flow rate and maintaining a continuous flow through the system. Once both sampling chambers were filled, the MODE valves were switched from ModeIII back to Mode-II, such that the fluid element by-passes the sample chambers and only traverses through the columns. At this time, the VENT valves were switched from closed to open, and the samples were taken from the sampling ports of both SC1 and SC2. Once both samples were collected, the VENT valves were closed and the MODE valves were once again switched back to pulse in Mode-III until SC1 and SC2 were filled. This sequence was repeated at approximately 5-minute increments between Mode-II and Mode-III, for a total analysis period of just over 3-hours. The prototype was secured to a sturdy, level frame that included supporting clamps for the packed fixed-bed columns and a removable sampling chamber lock mechanism for maintenance accessibility. 


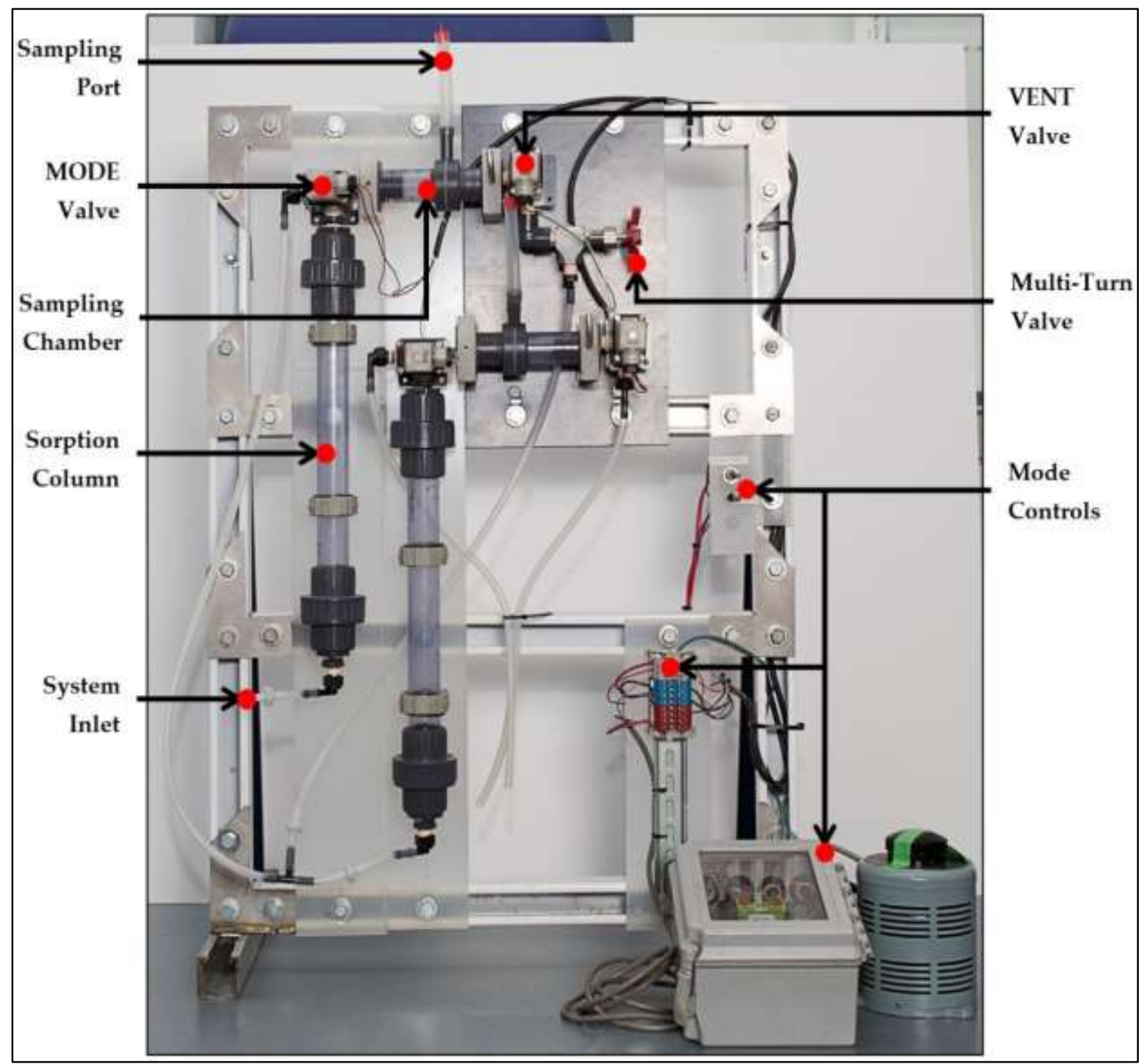

Figure 7.2 Image of Automated Sorption System Prototype Design (adapted from Ciosek and Luk, 2017d)

It is important to note that the sample chambers were fabricated to a $30-\mathrm{mL}$ capacity, to ensure that the $25-\mathrm{mL}$ required volume is attained, to be filtered for dilution in preparation of ICP-AES analysis. This influences the time to collect the sample volume, based on the selected pump flow rate of this study. Also, the spacing of the prototype components influences the tubing connection lengths. The dual-column sorption system design presented in Figure $\mathbf{7 . 1}$ and Figure 7.2 provides the opportunity to analyze higher flow rates and/or prolonged sample collection in Mode-III in future research endeavours. 


\subsection{Quality Control (QC) Protocol}

For all analytical sessions, the triplicate concentration of the median standard $(50 \mathrm{mg} / \mathrm{L})$ detected an average of $49.26 \mathrm{mg} / \mathrm{L}$, and is within $5 \%$ of the known value; the percent relative standard deviation (\%-RSD) reported an average of $0.433 \%$, which is well within the $\leq 3 \%$ limit; the correlation coefficient of each HMI analyte primary wavelength generated an average of 0.999977 , which is very close to unity.

The multi-component stock was created by diluting the respective HMI nitrate salts of three 1-L stock solutions, acidified to a $\mathrm{pH}$ of $2.0 \pm 0.1$, and then re-combined. These 3 stocks (denoted as $\mathrm{X}, \mathrm{Y}, \mathrm{Z}$ ) were diluted by one $50 \%$ step to be within the $0-100 \mathrm{mg} / \mathrm{L}$ calibration range, analyzing each separately and combined (denoted as $[\mathrm{M}]$ ). The five metals in a multicomponent system of $2.0 \mathrm{meq} / \mathrm{L}$ per metal corresponded to concentrations of approximately 207 $\mathrm{mg} / \mathrm{L}$ for $\mathrm{Pb}^{2+}, 64 \mathrm{mg} / \mathrm{L}$ for $\mathrm{Cu}^{2+}, 37 \mathrm{mg} / \mathrm{L}$ for $\mathrm{Fe}^{3+}, 59 \mathrm{mg} / \mathrm{L}$ for $\mathrm{Ni}^{2+}$, and $65 \mathrm{mg} / \mathrm{L}$ for $\mathrm{Zn}^{2+}$, respectively. The consistency in stock preparation is demonstrated in Table 7.1.

Table 7.1 ICP-AES Generated Multi-Component Stock Concentration.

\begin{tabular}{ccccc}
\hline Sample ID & Analyte & Int (Corr) & RSD (Corr Int) & Conc (Calib) (mg/L) \\
\hline \multirow{5}{*}{ M-X } & $\mathrm{Cu} 327.393$ & $188,070.71$ & 0.27 & 34.26 \\
& $\mathrm{Fe} 238.204$ & $79,641.94$ & 0.39 & 19.18 \\
& $\mathrm{Ni} 231.604$ & $41,071.22$ & 0.50 & 29.81 \\
& $\mathrm{~Pb} 220.353$ & $32,330.16$ & 0.52 & 105.28 \\
& $\mathrm{Zn} 206.200$ & $55,015.91$ & 0.38 & 32.31 \\
\hline \multirow{5}{*}{$\mathrm{M}-\mathrm{Y}$} & $\mathrm{Cu} 327.393$ & $186,885.03$ & 0.74 & 34.04 \\
& $\mathrm{Fe} 238.204$ & $79,083.95$ & 0.90 & 19.04 \\
& $\mathrm{Ni} 231.604$ & $40,721.53$ & 0.48 & 29.55 \\
& $\mathrm{~Pb} 220.353$ & $31,973.87$ & 0.31 & 104.12 \\
& $\mathrm{Zn} 206.200$ & $54,758.60$ & 1.09 & 32.16 \\
& $\mathrm{Cu} 327.393$ & $202,742.71$ & 0.91 & 36.93 \\
$\mathrm{M}-\mathrm{Z}$ & $\mathrm{Fe} 238.204$ & $85,771.53$ & 1.02 & 20.65 \\
& $\mathrm{Ni} 231.604$ & $44,652.28$ & 3.73 & 32.41 \\
& $\mathrm{~Pb} 220.353$ & $35,199.76$ & 3.77 & 114.63 \\
& $\mathrm{Zn} 206.200$ & $60,176.84$ & 4.12 & 35.34 \\
\hline \multirow{5}{*}{$\mathrm{M}[\mathrm{M}]$} & $\mathrm{Cu} 327.393$ & $192,776.82$ & 0.63 & 35.11 \\
& $\mathrm{Fe} 238.204$ & $81,419.80$ & 0.80 & 19.60 \\
& $\mathrm{Ni} 231.604$ & $41,667.28$ & 0.38 & 30.24 \\
& $\mathrm{~Pb} 220.353$ & $32,738.45$ & 0.40 & 106.61 \\
& $\mathrm{Zn} 206.200$ & $56,170.16$ & 0.94 & 32.99 \\
\hline
\end{tabular}

The average diluted concentrations of the $\mathrm{X}, \mathrm{Y}$, and $\mathrm{Z}$ influent stocks for $\mathrm{Cu}^{2+}, \mathrm{Fe}^{3+}, \mathrm{Ni}^{2+}$, $\mathrm{Pb}^{2+}$, and $\mathrm{Zn}^{2+}$ are $70.15,39.25,61.18,216.02$, and $66.54 \mathrm{mg} / \mathrm{L}$, respectively. The diluted concentration of the $\mathrm{M}$ influent stock for $\mathrm{Cu}^{2+}, \mathrm{Fe}^{3+}, \mathrm{Ni}^{2+}, \mathrm{Pb}^{2+}$, and $\mathrm{Zn}^{2+}$ are 70.22, 39.21, 60.48, 
213.2, and $65.98 \mathrm{mg} / \mathrm{L}$, respectively. A maximum difference of $2.80 \mathrm{mg} / \mathrm{L}$, equivalent to $1.30 \%$, is detected for the $\mathrm{Pb}^{2+}$ stock. Also, the corresponding $\mathrm{HMI}$ concentrations in $\mathrm{mg} / \mathrm{L}$ are comparable to the theoretically expected values, based on the selected total $10 \mathrm{meq} / \mathrm{L}$ initial concentration; only a $0.05 \%$ difference between the average of all initial concentrations of the theoretical and combined $\mathrm{M}$ stock is detected. Overall, this demonstrates that strong quality control has been implemented.

\subsection{Analysis}

\subsubsection{Preliminary Batch-Mode Trends}

Detailed analysis on the selected HMIs of this study was conducted by Ciosek and Luk (2017a; 2017c) in batch-mode configuration (as presented in Chapter 5 and 6 of the Dissertation document), consisting of a synthetic nitrate salt solution at $10 \mathrm{meq} / \mathrm{L}$ total concentration, acidified to a $\mathrm{pH}$ of 2 by concentration $\mathrm{HNO}_{3}$ acid, with a zeolite dosage of $4 \mathrm{~g}$ per 100-mL HMI solution. The aqueous solution was agitated within a contact period 180 minutes by means of a triple-eccentric drive orbital shaker operating at $400 \mathrm{r} / \mathrm{min}$ set to $22^{\circ} \mathrm{C}$. The five (5) HMIs were methodically combined in single-, dual-, triple-, and multi-component systems. Elemental analysis by ICP-AES concluded that after 3 hours, a total HMI uptake of $0.0986 \mathrm{meq} / \mathrm{g}$ is achieved the multi-component system. The percent removal of $\mathrm{Pb}^{2+}, \mathrm{Cu}^{2+}, \mathrm{Fe}^{3+}, \mathrm{Ni}^{2+}$ and $\mathrm{Zn}^{2+}$ are $94.0,21.9,56.2,9.10$, and $16.5 \%$, respectively. The zeolite's preference among the HMIs is demonstrated by the selectivity series, which was established as $\mathrm{Pb}^{2+}>>\mathrm{Fe}^{3+}>\mathrm{Cu}^{2+}>\mathrm{Zn}^{2+}>$ $\mathrm{Ni}^{2+}$. One of the objectives of this current study is to investigate how these HMIs interact and affect the removal uptake in a continuous-mode, dual-column flow settling.

\subsubsection{Automated Column Sorption System}

\subsubsection{Sampling Sequence and Flow Rate}

Table 7.2 provides the timeline of modes in the system set-up sequence. Once the inlet tubing and check valve are primed, the pump starts to fill the inlet connection cavity. At full flow rate in Mode-I, it required approximately 8:39 min:s to travel from the base to the top of column 1 (C1). After approximately 3:40 min:s, sample chamber 1 (SC1) was filled, and Mode-II (circulation) was initiated while the first sample (C1-A) was collected. In the continuous flow of 
Mode-II, and it required approximately 8:27 min:s for the flow to travel from the base to the top of column 2 (C2). The flow was then switched to Mode-III (pulse), which divides the flow to fill both sample chambers SC1 and SC2. Once the 30-mL volumes were filled, Mode-III is switched back to Mode-II and the samples C1-B and C2-B were collected at 42:50 min:s.

Table 7.2 Sorption System Set-Up Sequence

\begin{tabular}{|c|c|c|c|}
\hline MODE & Function & Flow Description & Time (min:s) \\
\hline \multirow{3}{*}{ I } & \multirow{3}{*}{ Fill C1 } & Primed Inlet to $\mathrm{C} 1 \mathrm{Base}$ & $2: 26$ \\
\hline & & C1 Base to C1 Top & 11:05 \\
\hline & & C1 Top to SC1 Drip & $15 \cdot 10$ \\
\hline \multirow{4}{*}{ II } & Fill SC1 & Sample C1-A & $18: 50$ \\
\hline & \multirow{3}{*}{ Fill C2 } & C2 Inlet to C2 Base & 24:08 \\
\hline & & C2 Base to C2 Top & $32: 35$ \\
\hline & & C2 Top to SC2 Drip & \multirow[t]{2}{*}{$36: 14$} \\
\hline III & \multirow{2}{*}{ Fill $\mathrm{SC} 1$ and $\mathrm{SC} 2$} & & \\
\hline $\begin{array}{l}\text { II } \\
\text { III }\end{array}$ & & Sample C1-B and C2-B & $\begin{array}{l}42: 50 \\
48: 04\end{array}$ \\
\hline $\begin{array}{l}\text { III } \\
\text { II }\end{array}$ & Fill SC1 and SC2 & Sample C1-1 and C2-1 & $54: 27$ \\
\hline
\end{tabular}

Once the system is set-up, there is an orderly switch between Mode-II (circulation) and Mode-III (pulse). Table 7.3 summarizes this sampling sequence. Altogether, there are twentynine 30-mL samples collected throughout the analysis period. During the system set-up, the collection of the first sample (C1-A) was followed by the second column 1 sample (C1-B) and first column 2 sample (C2-B). The orderly sequence began at the collection of $\mathrm{Cx}-1$ (48:04 min:s), for a total of two samples for each of the thirteen (13) runs. A total waste (TW) sample in the collection basin of the sorption system was also collected half-way through sampling (115:45 $\min : \mathrm{s})$ and at the end of the analysis period (195:00 min:s). The final influent stock and total effluent volumes were approximately $1.45-\mathrm{L}$ and $550-\mathrm{mL}$, respectively. 
Table 7.3 Sorption System Sampling Sequence

\begin{tabular}{|c|c|c|c|c|}
\hline \multirow{2}{*}{ Sample } & \multirow{2}{*}{ MODE } & \multirow{2}{*}{ Start Time (min:s) } & \multicolumn{2}{|c|}{ End Time (min:s) } \\
\hline & & & SC1 & SC2 \\
\hline \multirow{2}{*}{ C1-A } & I & $15: 10$ & $18: 50$ & - \\
\hline & II & $18: 50$ & & \\
\hline \multirow[b]{2}{*}{$\mathrm{Cx}-\mathrm{B}$} & III & $36: 40$ & $42: 42$ & $42: 49$ \\
\hline & II & $42: 50$ & & \\
\hline \multirow{2}{*}{$\mathrm{Cx}-1$} & III & $48: 04$ & $54: 10$ & $54: 27$ \\
\hline & II & $54: 27$ & & \\
\hline \multirow{2}{*}{$\mathrm{Cx}-2$} & III & $59: 39$ & $66: 15$ & $66: 34$ \\
\hline & II & $66: 34$ & & \\
\hline \multirow{2}{*}{$\mathrm{Cx}-3$} & III & $71: 37$ & $77: 55$ & 78:19 \\
\hline & II & $78: 20$ & & \\
\hline \multirow{2}{*}{$\mathrm{Cx}-4$} & III & $83: 24$ & $89: 56$ & $89: 56$ \\
\hline & II & $89: 57$ & & \\
\hline \multirow{2}{*}{$\mathrm{Cx}-5$} & III & $95: 05$ & $101: 32$ & 101:45 \\
\hline & II & $101: 46$ & & \\
\hline \multirow{2}{*}{ Cx-6 } & III & 106:52 & $112: 53$ & 113:10 \\
\hline & II & $113: 11$ & & \\
\hline TW1 & & $115: 45$ & & \\
\hline \multirow{2}{*}{$\mathrm{Cx}-7$} & III & 118:10 & $123: 45$ & $123: 56$ \\
\hline & II & $123: 57$ & & \\
\hline \multirow{2}{*}{$\mathrm{Cx}-8$} & III & 129:00 & 136:07 & 136:19 \\
\hline & II & $136: 20$ & & \\
\hline \multirow{2}{*}{ Cx-9 } & III & $141: 22$ & $147: 29$ & $147: 34$ \\
\hline & II & $147: 35$ & & \\
\hline \multirow{2}{*}{$\mathrm{Cx}-10$} & III & $152: 40$ & $158: 35$ & $158: 45$ \\
\hline & II & $158: 46$ & & \\
\hline \multirow{2}{*}{$\mathrm{Cx}-11$} & III & $163: 45$ & 169:40 & $169: 45$ \\
\hline & II & $169: 46$ & & \\
\hline \multirow{2}{*}{$\mathrm{Cx}-12$} & III & 174:50 & 181:06 & 181:04 \\
\hline & II & 181:10 & & \\
\hline \multirow{2}{*}{$\mathrm{Cx}-13$} & III & $186: 11$ & 192:08 & $192: 36$ \\
\hline & II & \multicolumn{3}{|c|}{ PUMP OFF } \\
\hline TW2 & & $195: 00$ & & \\
\hline
\end{tabular}

The flow patterns were continuous and methodically kept consistent throughout the analysis period. Once samples C1-B and C2-B were collected, an average time of 6:26 min:s passed to switch from Mode-III to Mode-II, and 5:05 min:s from Mode-II to Mode-III. When the flow is divided in Mode-III, the average sampling acquisition time of 6:19 min:s was required to fill the $30-\mathrm{mL}$ chambers, which was then collected for the filtering and dilution of the $25-\mathrm{mL}$ subsample. The adjustable bi-stable timer at an approximate 50\% duty setting automatically toggles the pulsing in Mode-III, to maintain a relatively consistent division of flow between the two columns, creating partial diversion to the two sampling chambers. This is demonstrated in relation to the start and end times of the sampling sequence. 
It is important to note that the first sorption column (C1) received a continuous inlet flow rate, as observed by the Mode-I filling rate of $8.18 \mathrm{~mL} / \mathrm{min}$ for $\mathrm{SC} 1$ sample C1-A. Immediately after SC1 was filled and before 30-mL collection, the switch to Mode-II diverted the flow to begin filling the second sorption column (C2). Once both columns were filled, the flow was divided in Mode-III at the top outlet of $\mathrm{C} 1$, between $\mathrm{SC} 1$ and $\mathrm{SC}$, while maintaining consistent contact throughout the system. Again, during Mode-III, C1 received the same inlet flow rate, but the sampling chambers $\mathrm{SC} 1$ and $\mathrm{SC} 2$ received this division of flow. It is column $\mathrm{C} 2$ that received a variable flow rate during the analysis period, set by the adjustable division timer. Based on the filling start time (36:40 min:s) of C1-B and C2-B, and the end time of collection (192:36 min:s) for C1-13 and C2-13, a geometric mean flow rate between Mode-II and Mode-III in C2 is established as $5.39 \mathrm{~mL} / \mathrm{min}$. With these unique flow rates recognized for both columns, their corresponding EBCTs are established by Equation 7.4 to yield:

$$
\begin{gathered}
\operatorname{EBCT}_{1}=\frac{d^{2} \pi \mathrm{H}}{4\left(\mathrm{Q}_{\mathrm{C} 1}\right)}=\frac{(2.61 \mathrm{~cm})^{2} \pi(30.48 \mathrm{~cm})}{4\left(8.18 \mathrm{~cm}^{3} / \mathrm{min}\right)} \cong 20: 00 \mathrm{~min}: \mathrm{s} \\
\operatorname{EBCT}_{2}=\frac{\mathrm{d}^{2} \pi \mathrm{H}}{4\left(\mathrm{Q}_{\mathrm{C} 2, \mathrm{AVG}}\right)}=\frac{(2.61 \mathrm{~cm})^{2} \pi(30.48 \mathrm{~cm})}{4\left(5.39 \mathrm{~cm}^{3} / \mathrm{min}\right)} \cong 30: 20 \mathrm{~min}: \mathrm{s}
\end{gathered}
$$

As reported by Reed et al. (1996) in the study of GAC column removal of lead, the void volume in columns is typically $\sim 45 \%$, which corresponds to a true residence time between the solution being treated and the sorbent particles of approximately double the EBCT. For a given sorbent material, the optimum EBCT occurs at which point no further improvement in column process efficiency (i.e., ๆ) occurs (Reed et al., 1996). With that being said, due to the relatively slow kinetics of zeolites, long residence times are required. Any solution volume-element in contact with a given zeolite bed layer is for only a limited time period, which is usually insufficient to reach the equilibrium state. The failure of zeolite to attain local equilibrium causes a lower uptake of HMIs from solution (Stylianou et al., 2007b). The detention time that the fluid element is in contact with the fixed-bed per sorption column is a result of the flow rate selected in this present study. This trend between the columns provides insight into the overall treatment availability of the zeolite material in this very unique configuration. 


\subsubsection{2. $\quad$ Acidity Levels}

Table 7.4 presents the $\mathrm{pH}$ levels of the effluent for both columns, of equally distributed selected time-step checkpoints of Cx-3, Cx-6, Cx-9, and Cx-13. When the acidified influent stock is combined to a 3-L volume, the average multi-component $\mathrm{MM} \mathrm{pH}$ has a value of 1.90. By maintaining a very low initial $\mathrm{pH}$ and the use of highly soluble nitrate salts, the precipitation of the HMIs is avoided. Additional trials verified that the filtered and unfiltered HMI influent stock concentrations are the same, indicating both effective dilution practices and complete solubility.

Table 7.4 The $\mathrm{pH}$ Levels of Selected Sorption Column Samples

\begin{tabular}{|c|c|c|}
\hline \multirow{2}{*}{ Sample } & \multicolumn{2}{|c|}{ pH Level } \\
\hline & SC1 & SC2 \\
\hline C1-A & 6.34 & - \\
\hline$C x-3$ & 4.79 & 6.84 \\
\hline $\mathrm{Cx}-6$ & 3.99 & 6.72 \\
\hline TW1 & \multicolumn{2}{|c|}{6.05} \\
\hline $\mathrm{Cx}-9$ & 3.86 & 6.33 \\
\hline $\mathrm{Cx}-13$ & 3.60 & 5.76 \\
\hline TW2 & \multicolumn{2}{|c|}{5.44} \\
\hline
\end{tabular}

As the sample traverses through the first column $\mathrm{C} 1$, the $\mathrm{H}^{+}$ions are captured by the zeolite, resulting in an increase in the $\mathrm{pH}$ to 6.34 from the first sample $\mathrm{C} 1-\mathrm{A}$. There is an interesting observation between the columns' $\mathrm{pH}$ levels, which is a direct reflection of the zeolite's removal capacity for both the HMIs of interest and the competitive $\mathrm{H}^{+}$ions in solution. The $\mathrm{pH}$ level gradually decreases in both columns, with the levels of $\mathrm{C} 2$ being slightly greater than that of $\mathrm{C} 1$. The total waste (TW) collects in the effluent basin throughout the analysis period, and its $\mathrm{pH}$ level decreases from the half-way check point of 6.05 (115:45 min:s) to 5.44 at the final collection (195:00 min:s). This is a clear indication that the zeolite capacity is becoming exhausted for the competing $\mathrm{H}^{+}$ions, as well as hindering the sorption process of the HMIs.

Zeolites are able to neutralize the solution acidity; when the point of saturation is reached, the ability of zeolite to neutralize decreases and the $\mathrm{pH}$ of the solution decreases back towards its initial pH level (Kocasoy and Sahin, 2007). This is also demonstrated by the research conducted by Vukojevic Medvidovic et al. (2006); the pH values changed during the uptake process, following the opposite shape of the typical breakthrough curves. At breakpoint, a drastic change in the $\mathrm{pH}$ occurred, which corresponded with a rapid $\mathrm{Pb}^{2+}$ concentration increase. The maximum 
$\mathrm{pH}$ is reached at the breakthrough point, due to the absence of HMIs in the effluent. The minimum $\mathrm{pH}$ is reached at the exhaustion point, due to the increase of the concentration of HMIs in the effluent and due to their hydrolysis in solution. After the exhaustion point, the $\mathrm{pH}$ is constant (Nuic et al., 2013). These findings suggest that the continuous monitoring of $\mathrm{pH}$ levels is important and considerably contributes to the prediction of breakthrough and exhaustion points (Vukojevic Medvidovic et al., 2006; Stylianou et al., 2007b; Nuic et al., 2013); in order to monitor the progress of the service life and inevitably regeneration (adsorption/desorption cycles), both of which are very significant for practical industrial applications (Vukojevic Medvidovic et al., 2006).

It should be noted that while low $\mathrm{pH}$ levels prevent precipitation, the competitive $\mathrm{H}^{+}$ions present would hinder the sorption of HMIs. Therefore, it is to be expected that future field installations for the treatment of industrial wastewater effluents should potentially demonstrate even higher removal efficiencies. However, care should be taken in the design of industrial applications to incorporate pre-treatment processes to reduce particulates prior to applying the waste to any sorption system, to avoid flow obstruction in the sorption columns.

\subsubsection{Hydraulic Conductivity Considerations}

Following HMI uptake analysis, the columns were drained to sit overnight. The standard test method for permeability of granular soils (constant head) (ASTM D2434-68) was adopted to determine the variance in the overall hydraulic conductivity between the sorption columns. The hydraulic coefficient of permeability is given by adapting the standard test in the following relationship:

$$
\mathrm{k}_{\mathrm{T}}=\frac{\mathrm{V}_{\mathrm{C}} \cdot \mathrm{L}}{\mathrm{A}_{\mathrm{c}} \cdot \mathrm{H}_{\mathrm{C}} \cdot \mathrm{T}}
$$

where $\mathrm{k}_{\mathrm{T}}$ is the coefficient of permeability (in $\mathrm{cm} / \mathrm{s}$ ), $\mathrm{V}_{\mathrm{C}}$ is the quantity of water that has discharged from the column and collected (in $\mathrm{cm}^{3}$ ), $\mathrm{L}$ is the column height (in $\mathrm{cm}$ ), $\mathrm{A}_{\mathrm{c}}$ is the column cross-sectional area $\left(\right.$ in $\left.\mathrm{cm}^{2}\right), \mathrm{H}_{\mathrm{C}}$ is the constant head of water on the column or the vertical distance between the feed head level and the column overflow level (in $\mathrm{cm}$ ), and $\mathrm{T}$ is the time required (in seconds) to collect the $\mathrm{V}_{\mathrm{C}}$ volume. With a plumb tank clamp support system, the water was fed in upflow mode from its base. The collection volume $\left(\mathrm{V}_{\mathrm{C}}\right)$ was set to $50-\mathrm{mL}$ by a graduated cylinder, with a column height (zeolite bed depth) and cross-sectional area of 30.48 
$\mathrm{cm}$ and $5.37 \mathrm{~cm}^{2}$, respectively. Based on an $18^{\circ} \mathrm{C}$ detected water temperature, the viscosity correction factor of $\mathrm{n}_{\mathrm{T}} / \mathrm{n}_{20}=1.0508$ is applied to reveal the hydraulic conductivity of columns $\mathrm{C} 1$ and $\mathrm{C} 2$ as $4.08 \times 10^{-4} \mathrm{~m} / \mathrm{s}$ and $3.89 \times 10^{-4} \mathrm{~m} / \mathrm{s}$, respectively. With a $4.84 \%$ difference to the average between the columns, this demonstrates a consistency in the overall executed compaction method.

\subsubsection{Heavy Metallic Ion Concentration Analysis}

Table 7.5 provides the results of the HMI concentrations $\left(C_{t}\right)$ in both sorption columns based on triplicate readings obtained by the ICP-AES software. The percent removal $(\% \mathrm{R})$ values are presented with respect to the $2.0 \mathrm{meq} / \mathrm{L}$ influent concentrations of each HMI. During the experimental sequence of sample collection, C2-11 was lost due to improper handling when transferring from the sampling port to filtration at 169:45 min:s. However, this sample is of a lower HMI concentration and the overall removal trend has been well-established by the time this sample is collected. The first major observation is that throughout the analysis period, $\mathrm{Pb}^{2+}$ is not detected in both column effluents as well as the total waste, indicating a complete removal of the ion. The $\mathrm{C} 1-13$ sample for $\mathrm{Cu}^{2+}, \mathrm{Fe}^{3+}$, and $\mathrm{Zn}^{2+}$ reports a removal of $18.1,82.5$, and $10.7 \%$, respectively, from the first column. The dual-column configuration provides a substantial improvement on the removal as observed with the second pass in sample C2-13, to achieve a final removal of $\mathrm{Cu}^{2+}, \mathrm{Fe}^{3+}$, and $\mathrm{Zn}^{2+}$ of $80.1,99.98$, and $51.5 \%$, respectively. This improvement is also attributed to the unique feeding rate and design of the second column $\mathrm{C} 2$; the additional EBCT of approximately ten minutes is available for the sorption process to occur as well as the slightly higher $\mathrm{pH}$ levels (and therefore lower presence of competitive $\mathrm{H}^{+}$ions). The final total waste (TW2) effluent concentrations report very good removal for all ions except for $\mathrm{Ni}^{2+}$, with a removal of $49.0 \%$. This removal trend is also consistent with the batch analyses conducted by Ciosek and Luk (2017a; 2017c). This is significant, as it proves that results from complex experimental batch studies, which are in high abundance, are useful in providing information on the sorption performance (i.e., removal efficiency, selectivity, and kinetics) in industrial applications where the process is run in a continuous flow-feeding configuration. In summary, the results demonstrate for the first time the effectiveness of multiple HMIs sorption by zeolite in a dual-column system with continuous flow. 
Table 7.5 The HMI Concentration (meq/L) and Percent Removal (\%R) in the Sorption Columns

\begin{tabular}{|c|c|c|c|c|c|c|c|c|c|c|c|}
\hline \multirow{3}{*}{\multicolumn{2}{|c|}{ Sample }} & \multicolumn{10}{|c|}{ HMI } \\
\hline & & \multicolumn{2}{|c|}{$\mathrm{Cu}^{2+}$} & \multicolumn{2}{|c|}{$\mathrm{Fe}^{3+}$} & \multicolumn{2}{|c|}{$\mathrm{Ni}^{2+}$} & \multicolumn{2}{|c|}{$\mathbf{P b}^{2+}$} & \multicolumn{2}{|c|}{$\mathbf{Z n}^{2+}$} \\
\hline & & meq/L & $\% \mathrm{R}$ & meq/L & $\% \mathrm{R}$ & meq/L & $\% \mathbf{R}$ & meq/L & $\% \mathbf{R}$ & meq/L & $\% \mathbf{R}$ \\
\hline \multirow{15}{*}{ C1 } & C1-A & 0.000 & 100 & 0.000 & 100 & 0.007 & $99.7^{\mathrm{BP}}$ & 0.0003 & 99.98 & 0.002 & $99.9^{\mathrm{BP}}$ \\
\hline & C1-B & 0.129 & $93.6^{\mathbf{B P}}$ & 0.000 & 100 & 0.911 & 54.5 & 0.0006 & 99.97 & 0.541 & 72.7 \\
\hline & C1-1 & 0.517 & 74.2 & 0.000 & 100 & 1.483 & 25.9 & 0.0004 & 99.98 & 0.974 & 51.3 \\
\hline & C1-2 & 0.938 & 53.1 & 0.001 & 99.9 & 1.906 & 4.7 & 0.0006 & 99.97 & 1.320 & 34.0 \\
\hline & C1-3 & 1.221 & 38.9 & 0.011 & 99.5 & 2.116 & 0.00 & 0.0003 & 99.98 & 1.507 & 24.7 \\
\hline & C1-4 & 1.369 & 31.5 & 0.030 & 98.5 & 2.231 & 0.00 & 0.0006 & 99.97 & 1.622 & 18.9 \\
\hline & C1-5 & 1.431 & 28.5 & 0.052 & 97.4 & 2.269 & 0.00 & 0.0004 & 99.98 & 1.671 & 16.4 \\
\hline & C1-6 & 1.468 & 26.6 & 0.072 & 96.4 & 2.273 & 0.00 & 0.0005 & 99.97 & 1.703 & 14.8 \\
\hline & C1-7 & 1.584 & 20.8 & 0.102 & $94.9^{\mathbf{B P}}$ & 2.316 & 0.00 & 0.0005 & 99.98 & 1.816 & 9.20 \\
\hline & C1-8 & 1.563 & 21.9 & 0.118 & 94.1 & 2.199 & 0.00 & 0.0005 & 99.98 & 1.751 & 12.4 \\
\hline & C1-9 & 1.543 & 22.8 & 0.138 & 93.1 & 2.174 & 0.00 & 0.0004 & 99.98 & 1.730 & 13.5 \\
\hline & C1-10 & 1.5 & 21.4 & 0.167 & 91.6 & 2.134 & 0.00 & 0.0004 & 99.98 & 1.739 & 13.0 \\
\hline & C1-11 & 1.598 & 20.1 & 0.209 & 89.6 & 2.123 & 0.00 & 0.0004 & 99.98 & 1.752 & 12.4 \\
\hline & C1-12 & 1.604 & 19.8 & 0.268 & 86.6 & 2.096 & 0.00 & 0.0001 & 100 & 1.750 & 12.5 \\
\hline & C1-13 & 1.638 & 18.1 & 0.349 & 82.5 & 2.130 & 0.00 & 0.0002 & 99.99 & 1.786 & 10.7 \\
\hline \multirow{15}{*}{$\mathrm{C} 2$} & C2-B & & 100 & 0.0004 & 99.98 & 0.002 & 99.9 & 0.0003 & 99.99 & 0.002 & 99.9 \\
\hline & C2-1 & 0 . & 100 & 0.0003 & 99.98 & 0.002 & 99.9 & 0.0003 & 99.99 & 0.001 & 99.9 \\
\hline & C2-2 & 0.00 & 100 & 0.0003 & 99.98 & 0.012 & 99.4 & 0.0004 & 99.98 & 0.001 & 99.6 \\
\hline & $\mathrm{C} 2-3$ & 0.00 & 100 & 0.0002 & 99.99 & 0.046 & 97.7 & 0.0006 & 99.97 & 0.001 & 99.7 \\
\hline & $\mathrm{C} 2-4$ & 0.00 & 100 & 0.0002 & 99.99 & 0.131 & $93.4^{\mathrm{BP}}$ & 0.0002 & 99.99 & 0.001 & 99.9 \\
\hline & $\mathrm{C} 2-5$ & 0.00 & 100 & 0.0002 & 99.99 & 0.285 & 85.6 & 0.0002 & 99.99 & 0.009 & 99.6 \\
\hline & C2-6 & 0.00 & 100 & 0.0003 & 99.98 & 0.505 & 74.8 & 0.0003 & 99.99 & 0.049 & 97.6 \\
\hline & & 0.00 & 100 & 0.0002 & 99.99 & 0.835 & 58.3 & 0.0003 & 99.98 & 0.155 & $92.2^{\mathrm{BP}}$ \\
\hline & C2-8 & 0.004 & 99.8 & 0.0002 & 99.99 & 1.163 & 41.9 & 0.0003 & 99.99 & 0.312 & 84.4 \\
\hline & C2-9 & 0.029 & 98.6 & 0.0002 & 99.99 & 1.444 & 27.8 & 0.0004 & 99.98 & 0.455 & 77.2 \\
\hline & C2-10 & 0.085 & $95.8^{\mathbf{B P}}$ & 0.0003 & 99.99 & 1.675 & 16.3 & 0.0004 & 99.98 & 0.597 & 70.2 \\
\hline & C2-12 & 0.289 & 85.6 & 0.0002 & 99.99 & 2.126 & 0.00 & 0.0006 & 99.97 & 0.895 & 55.2 \\
\hline & $\mathrm{C} 2-13$ & 0.399 & 80.1 & 0.0003 & 99.98 & 2.198 & 0.00 & 0.0004 & 99.98 & 0.969 & 51.5 \\
\hline & TW1 & 0.0514 & 97.4 & 0.0004 & 99.98 & 0.3077 & 84.6 & 0.0004 & 99.98 & 0.1107 & 94.5 \\
\hline & TW2 & 0.1659 & 91.7 & 0.0057 & 99.72 & 1.0207 & 49.0 & 0.0000 & 100 & 0.4088 & 79.6 \\
\hline
\end{tabular}




\subsubsection{Breakthrough Curve, Capacity and Usage Rate Analysis}

The breakthrough curve is displayed in Figure 7.3, as a plot of the solute outlet concentration $\left(C_{t}\right)$ from Table 7.5 normalized to the inlet concentration $\left(C_{o}\right)$ (Vukojevic Medvidovic et al., 2006). This normalized ratio trend over the service time of analysis (Nuic et al., 2013; 2015) at which the sampling chambers (SC1, SC2) are extracted is presented for each of the five HMIs combined in the multi-component solution; for both the first sorption column (C1) and second sorption column (C2). The breakthrough point (BP) and exhaustion point (EP) of each HMI in each column are indicated. The first observation to be had is that the breakthrough curves of the first column $\mathrm{Cl}$ do not have a defined S-shape. However, in the second sorption column $\mathrm{C} 2$, the curves take on this typical shape. Vukojevic Medvidovic et al. (2006) points out that the shape-change may be attributed to an improved solid-solution phase contact for sorption to take place.

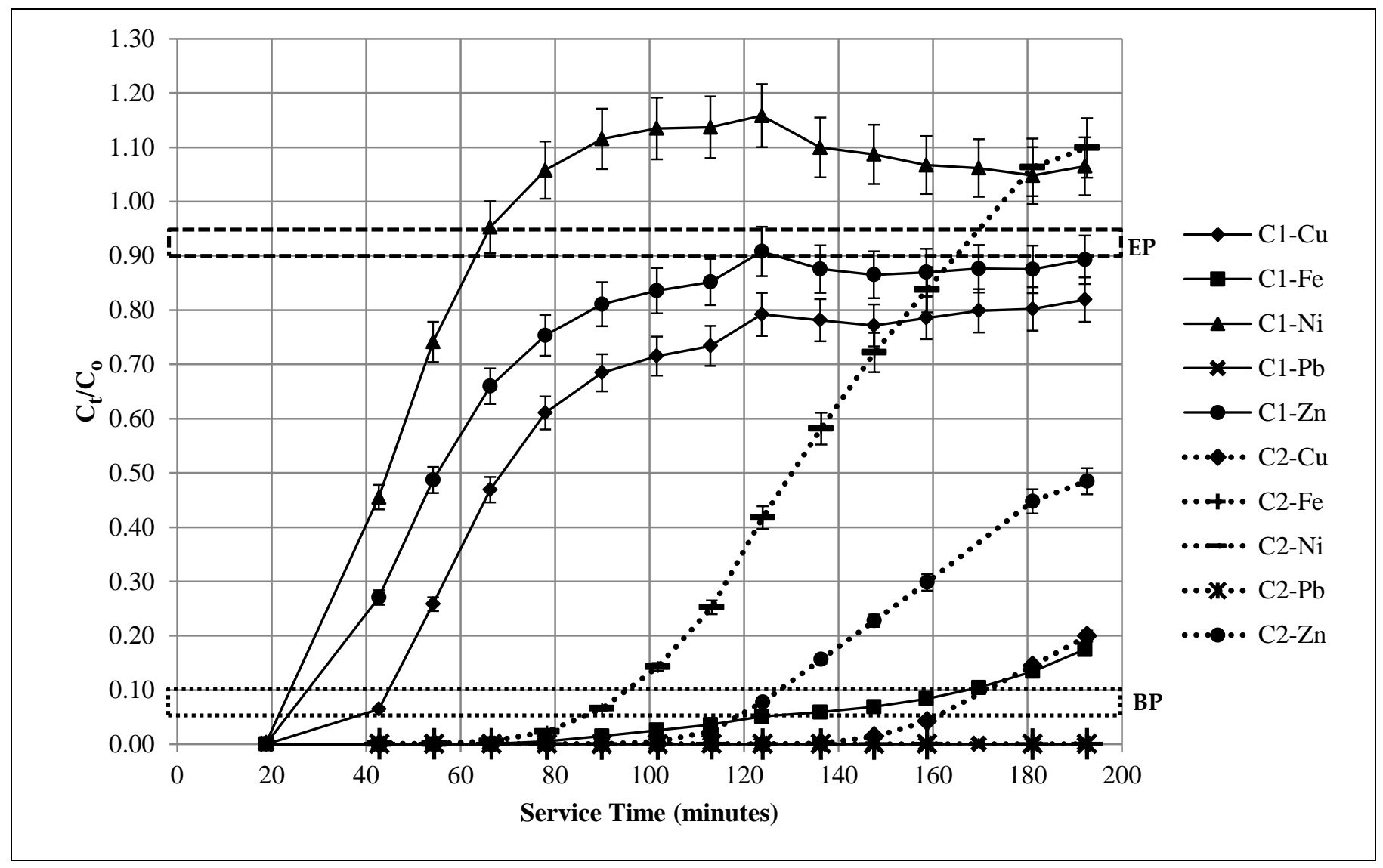

Figure 7.3 The Multi-Component System Breakthrough Curve 
The influent stock concentration of the $\mathrm{Ni}^{2+}$ ion is exceeded in the effluent solution, where the normalized $\mathrm{C}_{\mathrm{t}} / \mathrm{C}_{\mathrm{o}}$ ratio surpasses 1 to reach an approximate maximum of 1.16 at 125 minutes and 1.10 at 190 minutes of service time, in sorption columns $\mathrm{C} 1$ and $\mathrm{C} 2$, respectively. The final ratio readings plateau at the end of service to approximately 5-10\% of the value of 1, given the nature of this experimental investigation. The effluent concentration that overshoots the influent concentration translates to concentration wave extremes inside the column (Naja and Velosky, 2006). Nuic et al. (2013) investigated the breakthrough curves of $\mathrm{Pb}^{2+}$ and $\mathrm{Zn}^{2+}$ ions in dualcomponent solutions by natural zeolite; a similar trend was observed compared to this present study, where the $C_{t} / C_{o}$ ratio even reaches a value of 2 for one set of operation conditions. This is attributed to the displacement of the bound $\mathrm{Zn}^{2+}$ by the $\mathrm{Pb}^{2+}$ from the influent, which is supported by a lower breakthrough capacity and higher exhaustion capacity in favour of the $\mathrm{Pb}^{2+}$ ion, specifically (Nuic et al., 2013). It is important to note that the ion-exchange mechanism that attributes to the sorption process of HMIs transpires through the zeolite's framework of pores and channels. The presence of stronger binding HMIs, such as $\mathrm{Pb}^{2+}$, weaken the chemical bonds between the functional group on the surface of zeolite and the weaker HMIs, such as $\mathrm{Ni}^{2+}$ ions (Han et al., 2006). Given that zeolite demonstrates its highest preference towards $\mathrm{Pb}^{2+}$, sorption site availability has reached its threshold, which may cause the leaching out of ions that zeolite holds a lower preference towards during this process. Therefore, careful screening on the selectivity of HMIs by zeolite should be conducted prior to adaptation.

In summary, the major trends observed from the breakthrough curves are as follows:

1. The zeolite holds the greatest preference towards to $\mathrm{Pb}^{2+}$ ion, based on its complete removal throughout the analysis period;

2. The zeolite demonstrates the least preference towards the $\mathrm{Ni}^{2+}$ ion;

a. A more sudden breakpoint occurring after just $25 \mathrm{~min}$ and $90 \mathrm{~min}$ of service time in columns $\mathrm{C} 1$ and $\mathrm{C} 2$, respectively;

b. An approximate exhaustion point after just $65 \mathrm{~min}$ and $165 \mathrm{~min}$ of service time in columns $\mathrm{C} 1$ and $\mathrm{C} 2$, respectively;

3. The $\mathrm{Fe}^{3+}$ ion is removed entirely and sustained throughout the analysis period in $\mathrm{C} 2$, and;

4. The removal of both the $\mathrm{Cu}^{2+}$ and $\mathrm{Zn}^{2+}$ ions begin to plateau at 120 min of service time in $\mathrm{C} 1$, acting in parallel and do not reach the lower threshold of the exhaustion point in both columns throughout the analysis period. 
The breakthrough curves provide significant information from a perspective of sorption process performance, feasibility and optimization, which are vital for scaling-up the sorption system for industrial applications (Naja and Velosky, 2006; Inglezakis, 2010a).

The overall column performance efficiency and its relationship between breakthrough capacity $\left(\mathrm{C}_{\mathrm{BP}}\right)$ and exhaustion capacity $\left(\mathrm{C}_{\mathrm{EP}}\right)$ of each sorption column are unique to the individual HMIs selected. As observed in the data displayed Table 7.5 and the trends visualized in Figure 7.3, the EP is only attained by $\mathrm{Ni}^{2+}$; the remaining four HMIs have yet to reach this point due to the constraints of the 3-hour analysis period. Evidently, the optimization of future works would be to prolong the service time in order to quantify the overall columns' performance efficiency. Qualitatively speaking, for both columns' effluent and total waste (TW), the $\mathrm{Pb}^{2+}$ ion is completely removed throughout the analysis period, demonstrating the utmost efficiency; neither BP nor EP are attained. On the opposite end of the spectrum, the $\mathrm{Ni}^{2+}$ ion reaches exhaustion quite rapidly.

The volume of the effluent treated at breakthrough $\left(\mathrm{V}_{\mathrm{BP}}\right)$ is determined with the use of the mean flow rates of $8.18 \mathrm{~mL} / \mathrm{min}\left(\mathrm{Q}_{\mathrm{C} 1}\right)$ and $5.39 \mathrm{~mL} / \mathrm{min}\left(\mathrm{Q}_{\mathrm{C} 2}\right)$ for the sorption columns $\mathrm{C} 1$ and C2, respectively. These flow rates are applied to the time data of Table 7.3, at which BP (approximately $95 \%$ removal or $5 \%$ of the $2.0 \mathrm{meq} / \mathrm{L}$ influent concentration per HMI) is observed; as indicated by the superscript in Table 7.5. The 2:26 min:s time required for inlet priming to the base of $\mathrm{C} 1$ as well as the 24:08 min:s time observed for solute contact to the base of $\mathrm{C} 2$ are deducted from these BP times. As summarized in Table 7.6, the approximate effluent volumes treated at $\mathrm{BP}\left(\mathrm{V}_{\mathrm{BP}}\right)$ are provided for both sorption columns and each HMI selected; based on the zeolite mass in each bed $\left(\mathrm{m}_{\mathrm{Z}}\right)$ of $152.10 \mathrm{~g}$, Equation 7.2 and Equation 7.5 are employed to determine the corresponding breakthrough capacity $\left(\mathrm{C}_{\mathrm{BP}}\right)$ and usage rate $\left(\mathrm{V}_{\mathrm{U}}\right)$, respectively.

Table 7.6 System Breakthrough Point Performance

\begin{tabular}{ccccccc}
\hline \multirow{2}{*}{ HMI } & \multicolumn{3}{c}{ Sorption Column 1 } & \multicolumn{3}{c}{ Sorption Column 2 } \\
\cline { 2 - 7 } & $\mathrm{V}_{\mathrm{BP}, 1}(\mathrm{~L})$ & $\mathrm{C}_{\mathrm{BP}, 1}(\mathrm{meq} / \mathrm{g})$ & $\mathrm{V}_{\mathrm{U}, 1}(\mathrm{~g} / \mathrm{L})$ & $\mathrm{V}_{\mathrm{BP}, 2}(\mathrm{~L})$ & $\mathrm{C}_{\mathrm{BP}, 2}(\mathrm{meq} / \mathrm{g})$ & $\mathrm{v}_{\mathrm{U}, 2}(\mathrm{~g} / \mathrm{L})$ \\
\hline $\mathrm{Cu}^{2+}$ & 0.3295 & 0.0043 & 461.7 & 0.7257 & 0.0095 & 209.6 \\
$\mathrm{Fe}^{3+}$ & 0.9926 & 0.0131 & 153.2 & --- & --- & --- \\
$\mathrm{Ni}^{2+}$ & 0.1342 & 0.0018 & 1134 & 0.3547 & 0.0047 & 428.8 \\
$\mathrm{~Pb}^{2+}$ & -- & --- & --- & --- & --- & --- \\
$\mathrm{Zn}^{2+}$ & 0.1342 & 0.0018 & 1134 & 0.5380 & 0.0071 & 282.7 \\
\hline
\end{tabular}


The $\mathrm{Pb}^{2+}$ and $\mathrm{Fe}^{3+}$ ions are completely removed in sorption column $\mathrm{C} 2$, which essentially transforms the influent stock of a five HMI multi-component solution to a triple-component solution containing $\mathrm{Cu}^{2+}, \mathrm{Ni}^{2+}$, and $\mathrm{Zn}^{2+}$ ions within the 3-hour analysis period. The zeolite does not have to address the competition of the two preferred HMIs, which provides greater sorption site availability for the three HMIs remaining in solution. It is important to develop a relationship between the EBCT and usage rate. The time that the fluid element is in contact with the zeolite bed in sorption column $\mathrm{C} 2$ is ten minutes greater than the detention time in $\mathrm{C} 1$. This thereby demonstrates a stronger overall treatment availability in C2; reflected in the average 195\% increase of bed capacity at breakthrough and the average $63.9 \%$ decrease in usage rate of the zeolite between the columns; based on the trends observed for the $\mathrm{Cu}^{2+}, \mathrm{Ni}^{2+}$ and $\mathrm{Zn}^{2+}$ ions detected in solution.

Evidently, the columns' usage rate provides significant information into the operation and management required for this unique sorption system. It has a direct impact on the financial viability of performing either replacement (disposal) or regeneration (on- or off-site), and is affected by factors that include HMI influent concentration, zeolite bed depth, and flow rate. Research into other sorbent materials demonstrates that the order of usage rate is consistent with the sorption capacity (Othman et al., 2001). Due to the unique automated variable influent feeding rate and sampling technique proposed in this study, the usage rate and performance efficiency are very complex (Inglezakis et al., 2010a). However, the major removal trend of $\mathrm{Pb}^{2+}$ $>\mathrm{Fe}^{3+}>\mathrm{Cu}^{2+}>\mathrm{Zn}^{2+}>>\mathrm{Ni}^{2+}$ is well-established and supports previous results (Inglezakis et al., 2001b; Inglezakis et al., 2003; Inglezakis et al., 2004; Wingenfelder et al., 2005; Wang and Peng, 2010; Ciosek and Luk, 2017a; Ciosek and Luk, 2017c), providing significant validation of this design.

The use of natural zeolites as sorbents in industrial wastewater treatment and environmental management is motivated by the non-toxicity of these minerals, their abundant global availability, and economic feasibility. The removal and recovery processes of HMIs from aqueous solutions by natural zeolites take into consideration the regeneration potential of the zeolite bed to be reused in multiple cycles, as well as the use of the recovered metals (Sprynskyy et al., 2006) in applicable industrial applications. Metal processing effluents contain high concentrations of recoverable metals, triggering a movement towards technologies to recover 
these metals from industrial wastewater effluents, such as acid mine drainage waste (Zinck, 2005). The removal-regeneration-recovery process has the potential to generate additional revenue streams with the use of metals of value (Dinardo et al., 1991); such as the HMIs investigated in this innovative study.

\subsection{Conclusions}

This research has demonstrated the performance of natural zeolite (clinoptilolite) to remove multiple heavy metallic ions (HMIs) commonly found in industrial wastewater effluents. With the design and development of a novel dual-column sorption system, the lead $\left(\mathrm{Pb}^{2+}\right)$ ion is removed completely and sustained throughout the analysis period. The relationships between empty bed contact time (EBCT), breakthrough capacity, and usage rate are evident. The additional ten minutes of EBCT in the second sorption column contributes to an enhancement in overall removal for $\mathrm{Cu}^{2+}, \mathrm{Fe}^{3+}$, and $\mathrm{Zn}^{2+}$ by $75.7,99.9$, and $45.7 \%$, respectively, from the first sorption column. This improvement is also apparent in the greater breakthrough capacity and lower usage rate in the second column, and visualized in an improved S-shape to the characteristic breakthrough curve. Based on the multi-component influent stock of $10 \mathrm{meq} / \mathrm{L}$ total concentration, the second column demonstrates a removal of 99.98, 99.98, 80.1, 51.5, and $0.00 \%$, for $\mathrm{Pb}^{2+}, \mathrm{Fe}^{3+}, \mathrm{Cu}^{2+}, \mathrm{Zn}^{2+}$, and $\mathrm{Ni}^{2+}$, respectively; and the final cumulative collection of effluent reports a removal of $100,99.7,91.7,79.6$ and $49.0 \%$, for $\mathrm{Pb}^{2+}, \mathrm{Fe}^{3+}, \mathrm{Cu}^{2+}, \mathrm{Zn}^{2+}$, and $\mathrm{Ni}^{2+}$, respectively at the completion of the analysis period. These HMI sorption removal trends confirm the consistency between batch- and continuous-mode operations.

The modular design inventively incorporated a 'circulation-pulse' method to distribute the flow, rather than operating on a more commonly implemented fixed flow rate. With the consideration of this unique stock feed method, the findings of the service time and flow rate with respect to the removal trends are both interesting and significant. The potential for variable flow rate operation and automatic adjustable sampling in a packed fixed-bed dual-column sorption design reveals practicality for treatment applications. This study has provided greater insight into the immense potential that the natural mineral zeolite holds for the future of industrial wastewater treatment. 


\subsection{Future Works}

Forthcoming works in this research project include the investigation of regeneration cycles and service life, with further design development and optimization in various prospective configurations. In addition, the investigation from synthetic simple solute to actual wastewater effluent samples would provide a greater understanding of the system potential in practical engineering application. To be discussed in the Recommendations of the Dissertation document (with reference to Appendix E), an improvement to this system prototype is to place an emphasis on the removal-regeneration-recovery process in sorption removal technology development. 


\section{CONCLUSIONS AND RECOMMENDATIONS}

\subsection{Fundamental Conclusions}

The predominant presence of heavy metals in industrial wastewater effluents pose extreme environmental and health risks, owing to their highly toxic and dangerous nature, that is significantly complex to control. This Dissertation reports the fundamental findings of a fourphase research project to investigate the sorption removal capacity of natural zeolite of five heavy metallic ions (HMIs); lead $\left(\mathrm{Pb}^{2+}\right)$, copper $\left(\mathrm{Cu}^{2+}\right)$, iron $\left(\mathrm{Fe}^{3+}\right)$, nickel $\left(\mathrm{Ni}^{2+}\right)$, and zinc $\left(\mathrm{Zn}^{2+}\right)$; combined in various component systems. In keeping of existing literature and as 'proofof-concept', this research has demonstrated that natural zeolite is an effective sorbent material in the removal of HMIs commonly found in industrial wastewater under the testing conditions, and has contributed to the thought-provoking development of treatment system technology. The key findings to each phase of the project are provided in the following subsections.

\subsubsection{Effects of Operation Parameters}

Consistent with existing trends in literature, the removal of heavy metallic ions by natural zeolite increases with decreasing particle size, as well as increasing dosage, contact time, and set-temperature. The studied parameters influence the process in the order of: influent concentration $>$ heat pre-treatment level $>$ dosage $>$ particle size $>$ contact time $>$ settemperature. The removal efficiency order (or selectivity series) is consistent for all examined experimental conditions as: $\mathrm{Pb}^{2+}>>\mathrm{Fe}^{3+}>\mathrm{Cu}^{2+}>\mathrm{Zn}^{2+}>\mathrm{Ni}^{2+}$.

Based on preliminary investigations of existing critical performance trends (Acheampong et al., 2009; Motsi et al., 2009, 2011, Inglezakis, et al., 2002, 2003, 2004, 2005; Sprynskyy et al., 2006), the experimental conditions presented in Table 3.2 were established for the analyses of all operation parameters and conditions; as summarized in Table 4.1. The particle size and dosage operation parameters were investigated in single-component system combinations of all five HMIs, which are significant parameters that form the basis for the remaining conditions. The analysis of the influent concentration at a total $10 \mathrm{meq} / \mathrm{L}$ is a subset of the kinetic modelling investigations found in Chapter 6, while the $400 \mathrm{mg} / \mathrm{L}$ condition for each HMI was included and selected on the basis that when converting from meq/L to $\mathrm{mg} / \mathrm{L}$, this is the median range for a majority of the HMIs selected. The heat pre-treatment operation parameter was a stand-alone 
investigation, with a systematic increase from single- $[\mathrm{P}]$ to triple- $[\mathrm{T}]$ to multi- $[\mathrm{M}]$ component system HMI combinations, to quantitatively and qualitatively demonstrate the effects of heat on the zeolitic structure. The contact time conditions were to extrapolate from the 180 contact minutes established by preliminary investigations, which discusses the period at which sorption equilibrium is achieved with support of existing literature. Finally, the set-temperature conditions were set to develop a greater understanding of its influence of zeolite sorption selectivity.

Various factors (i.e., operation parameters, conditions) affect the overall removal efficiency, either positively or negatively. However, the efficiency may not be influenced by each factor individually. Unless the efficiency among all the factors is systematically arranged, the optimal combination of factors is not straightforwardly recognized. In order to reach the optimal combination of factors, those which positively influence the removal efficiency ought to be identified and investigate a refined combination of these factors with respect to the efficiency. Ideally, this aspect of the research project would be improved by investigating all six (6) operation parameters with all HMI component-system combinations. However, these combinations are fundamentally selected based on laboratory feasibility and hence, are the rationale towards the selection of the parameters presented.

\subsubsection{Lead Removal Capacity and Selectivity}

Zeolite cation exchange selectivity depends on the zeolite type, distance between anionic sites, cationic radii, hydrated cationic radii, and cationic hydration energy. The unhydrated ions are able to pass through the three-dimensional framework, but the hydrated ions may exchange only with faced obstacles. The $\mathrm{Pb}^{2+}$ ion has the smallest hydrated cationic radius and may pass effortlessly through this network. The cations with larger hydration energies prefer the sorbate (solution) phase, while the cations with the least hydration energies prefer the sorbent (zeolite) phase. As such, the lower hydration energy of $\mathrm{Pb}^{2+}$ implies that it loses its hydration shell more readily than other ions (Inglezakis et al., 2003); demonstrated by the high affinity and selectivity of clinoptilolite towards the $\mathrm{Pb}^{2+}$ ion (Oter and Akcay, 2007). In addition, the $\mathrm{Pb}^{2+}$ studied typically does not have a strong tendency to form strong complexes, and gives reasoning for its preferential uptake by natural zeolite (Inglezakis et al., 2004).

Under the testing conditions of this study, the methodical introduction of copper, iron, nickel and zinc to the component system decreases the overall uptake of the total HMIs in solution. 
However, as previous work suggests (Wingenfelder et al. 2005; Prasad and Mortimer, 2011), the cation competition does not affect the preferential uptake of zeolite for the $\mathrm{Pb}^{2+}$ ion.

\subsubsection{Kinetic Modelling Trends}

The accuracy of modelling prediction is dependent on the various open boundary conditions, model parameters selected, and numerical method(s) implemented. The study of sorption kinetics holds great importance in sorbent material assessment and subsequent application in wastewater treatment (Qiu et al., 2009); it defines the reaction pathways and uptake rates, along with residence times at which the equilibrium point is reached at the solid-solution interface. Both the pseudo-second-order (PSO) and intra-particle diffusion kinetic model demonstrated a very strong correlation of the experimental data; however, the PSO model demonstrated a stronger performance, and further contributed to the assessment of the zeolite mineral performance and the fundamental mechanisms associated with the sorption process under the testing conditions.

The variation in sorption kinetics is attributed to the specific crystalline structure (physical as well as chemical composition) of the sorbent material, and is controlled by various mechanisms (Helfferich, 1962; Bekkum et al., 1991; Kocaoba et al., 2007; Motsi et al., 2011); best understood with experimental data of the simultaneous sorption of the HMIs of interest, and an analysis on the uptake and rate of interference of these counter-ions in combination (Helfferich, 1962; Borandegi and Nezamzadeh-Ejhieh, 2015). Therefore, kinetic modelling is considered as a powerful tool to assess the performance of sorbent materials and to comprehend these fundamental mechanisms involved in the sorption process.

\subsubsection{Dual-Column Sorption System Performance}

This study successfully demonstrated the performance of natural zeolite to remove multiple heavy metallic ions (HMIs) commonly found in industrial wastewater effluent, with the HMI sorption removal trends confirming the consistency between batch- and continuous-mode configuration operations. The modular design of a novel dual-column sorption system inventively incorporated a unique stock feed 'circulation-pulse' method to distribute the flow. The lead $\left(\mathrm{Pb}^{2+}\right)$ ion was removed completely and sustained throughout the analysis period. The additional ten minutes of empty bed contact time (EBCT) in the second sorption column lends to the removal enhancement from the first sorption column; which demonstrated greater 
breakthrough capacity and lower usage rate. The relationships between EBCT, breakthrough capacity, and usage rate are significant, and the potential for variable flow rate operation and automatic adjustable controls in the packed fixed-bed dual-column sorption design reveals practicality for future treatment applications.

\subsection{Future Recommendations}

The ecosystem is a fragile network which requires a proactive rather than a reactive approach to dealing with industrial process pollution. Therefore, the advancement of treatment technologies is vital to minimizing the impact of pollutants on the environment. The selection of the most suitable treatment technology for inorganic metal-contaminated effluents is influenced by environmental impact and legislation/regulations, complacency and socio-economic constraints, existing process flow-schematics and site-specific logistics (i.e., technical applicability), lifecycle management and associated economics (cost-effectiveness, capital investment, operation and maintenance costs), overall water balance and climatic conditions, as well as wastewater effluent complexity (i.e., composition, $\mathrm{pH}$, initial metal concentration) and notably, the overall treatment performance compared to other technologies (Johnson and Hallberg, 2005; Adams, 2008; Barakat, 2011; Fu and Wang, 2011). In addition, the treatment system lifecycle is of increasingly great importance, with respect to disposal, the removalremediation-recovery cycle and the potential for financial return (Johnson and Hallberg, 2005). Lata et al. (2015) points out that the evaluation should be based on performance (removal efficiency) and cost, as well as the level of 'appropriateness', which reflects the robustness, quality and quantity output, (site-specific) condition suitability, and adaptability of a given treatment system. The design of a field-implemented prototype for the future must also take several technical complexities into account. A strong understanding must be had of all the biogeo-chemical and physical processes taking place within the effluent (Ritcey, 2005). The hydrologic parameters such as detention time, flow rate, and volume capacity for the development of the treatment unit are also significant (Kuyucak et al., 2013). Future optimization of the prototype would be to critically analyze these myriad of complex parameters, which will influence the overall treatment performance of the system, in order to practically and economically implement on an industrial scale. 
It is important to note that studies analyzing synthetic simple solute influent solutions have demonstrated greater removal performance compared to investigating actual wastewater (Motsi et al., 2009; Xu et al., 2010, 2013). This is largely due to the site-specific complexity (i.e., $\mathrm{pH}$ levels, HMI trace concentrations, various solution constituents). The study conducted by Prasad and Mortimer (2011) of actual acid mine drainage with fly ash and fly ash zeolite prompts the discussion of the importance of the solution $\mathrm{pH}$ level. The addition of the mineral to a relatively neutral $\mathrm{pH}$ solution may increase the $\mathrm{pH}$ level to reach the threshold of solubility of the metal hydroxides. This means that the removal may be partially attributed to precipitation (or sorption/co-precipitation) rather than just sorption (ion-exchange) (Wingenfelder et al. 2005; Prasad and Mortimer, 2011). This is echoed by the research conducted by Xu et al. (2014) using natural clinoptilolite as the sorbent material of an actual acid mine drainage sample; the $\mathrm{pH}$ level increase is caused by neutralization, attributing to the HMI removal primarily by precipitation, co-precipitation and adsorption. The proportions of interchangeable co-cations in the zeolite framework and those constituents in an actual sample that may hydrolyze have an impact on the overall presence of mineral species when in contact with the zeolite mineral. Xu et al. (2014) demonstrated in the study of adsorption/co-precipitation on Al-colloids that a final $\mathrm{pH}$ level of solution below 4 does not result in co-precipitates with colloids. This finding is in accordance with Inglezakis et al. (2003), whose synthetic simple influent solutions are acidified with nitric acid to a $\mathrm{pH}$ level of 2 , to avoid hydroxide precipitation during the removal of $\mathrm{Pb}^{2+}, \mathrm{Cu}^{2+}, \mathrm{Fe}^{3+}$ and $\mathrm{Cr}^{3+}$ by clinoptilolite (Xu et al., 2014); in keeping of the experimental design of the research presented in the Dissertation document, under the testing conditions. As seen in Table 7.4 of

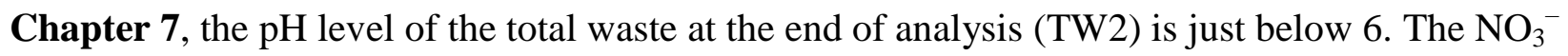
anions in the synthetic aqueous solution in this study do not influence the ion-exchange process, since they do not form any metal-anion complexes and do not hydrolyze in solution (Peric, 2004; Minceva et al., 2008) under these controlled conditions of this study. However, various anionic ligands, which include $\mathrm{NO}_{3}{ }^{-}, \mathrm{SO}_{4}{ }^{2}$, and $\mathrm{Cl}^{-}$, are present in actual AMD. As demonstrated by Xiu et al. (2014), $\mathrm{SO}_{4}{ }^{2-}$ is said to form inner-sphere complexes with active sites and to increase the net negative charge at the surface of the zeolite, encouraging the processes of metal adsorption as well as dealumination. Hence, further investigation of actual industrial wastewater effluent and the impacts of colloidal processes (although site-specific) are needed for the practical implementation of the system prototype. 
The synthetic simple solute solution used in this study was free of any suspended solids, and therefore, there was no need to fluidize/backwash the bed, during the analysis period under the testing conditions. However, with actual wastewater, the presence of suspended solids is another factor which influences the efficiency of a treatment system. The concentrations vary among industry, such as $4.1 \mathrm{mg} / \mathrm{L}$ for a copper production plant (Beyazit, 2013) or $22 \mathrm{mg} / \mathrm{L}$ for typical gold mine wastewater (Acheampong et al., 2009). The surfaces of suspended solids can occasionally acquire an electrical charge, much as dissolved species. This surface charge can either improve or impede the adsorption of ions from solution (Benjamin, 2010, p. 773). In addition to its influences in the chemisorption process (Benjamin, 2010, p. 774), suspended solids may also inhibit the hydraulic performance in the system. As a recommendation, clogging should be addressed in an established operation and maintenance plan following further optimization of the prototype design.

The natural zeolite clinoptilolite is considered as a strong candidate for the removal of HMIs from wastewater (Ersory and Celik, 2002; Alvarez-Ayuso et al., 2003). In addition to being a low-cost, globally abundant natural mineral (Kocasoy and Sahin, 2007), they hold the potential for regeneration, HMI recovery and reuse (Mohan and Chander, 2006; Lata et al., 2015) in relevant industrial applications. Metals cannot be destroyed and unless recovered, they become a recurring disposal problem. Consequently, an emphasis has shifted to treatment technologies which facilitate the recovery of metal pollutants; with economically attractive alternatives (Dinardo et al., 1991). In the ion-exchange study conducted by Riveros and Wong (1995), the extraction of $\mathrm{Sb}, \mathrm{Cd}, \mathrm{Cu}, \mathrm{Ni}$, and $\mathrm{Zn}$ from acid mine drainage solutions were analyzed in batchand continuous-mode (column tests). An annual cost of full neutralization to $\mathrm{pH}$ level of 11 followed by re-acidification to $\mathrm{pH}$ level of 7 was compared to lime neutralization to $\mathrm{pH}$ level of 6 followed by adsorption onto zeolite (clinoptilolite). Based on an all-year operation at a flow rate of $10,000 \mathrm{~m}^{3} /$ day and an initial $\mathrm{pH}$ level of 2.1 , the annual cost of lime was reduced from $\$ 1,560,375$ to $\$ 1,178,950$, and a total annual reduction in reagents of approximately $\$ 313,877.00$. There are various limitations in cost, performance and market readiness of existing industrial wastewater technologies. As such, the implementation of natural zeolite as an alternative presents additional benefit of metal recovery potential (Zinck et al., 2005). 
The regeneration/desorption of sorbent materials is a critical aspect in heavy metal removal and recovery, as it impacts the economic feasibility and service life of the treatment technology (Lata et al., 2015). The exhausted sorbent material is commonly exposed to thermal regeneration. This process usually causes a material loss of 10-20\% due to repeated attrition. Elution of the sorbate (i.e., extracting one material from another; washing the sorbent material with a solvent to remove the captured HMIs) with regeneration by a suitable reagent is a viable alternative to thermal regeneration (Mohan and Chander, 2006). Such regenerative agents include acids (i.e., $\mathrm{HCl}, \mathrm{H}_{2} \mathrm{SO}_{4}, \mathrm{HNO}_{3}$ ), alkalis and salts (i.e., $\mathrm{NaOH}, \mathrm{NaCl}$ ), as well as chelating agents (i.e., EDTA) and deionized distilled water (Lata et al., 2015). With regards to ultimate disposal, both the regenerated zeolite and recovered metals can be reused; such that the associated waste materials are returned to the environment with minimal impacts to the environment. This is the most environmentally-friendly way to move this technology into industry. If not, the metalcontaminated zeolite will need to be disposed of at special landfill sites. The development of an ideal eluent is required in order to address the fate of spent, metal-laden sorbent materials and to reduce the production of associated secondary pollutants (Lata et al., 2015); a factor in the optimization of the treatment system prototype.

It is important to note the potential for the derivation of multi-component sorption models. The column design and modelling simulation proposed by Jarvie et al. (2005) states that the type of sorbent material, EBCT, and bed configuration/operation major process design variables; which influence the steady state mass balance relationships to establish the usage rate of a given treatment system. In order to evaluate the performance, the influence of adsorbent type, solution chemistry and solute type must be investigated. The modelling approach simulated the effluent concentration profiles leaving the fixed-bed reactor, taking (external, intra-particle) mass transfer models into consideration. The Freundlich adsorption isotherm was selected to evaluate the equilibrium between the solid- and liquid-phase solute concentrations at the sorbent surface. The ultimate objective of the model development was to represent the dependence of adsorption capacity and kinetics upon time and bed-length, to be used to estimate effective diffusivities with enough precision to make crude design calculations; stressing the need for additional field data to develop confidence of the simulation in the future. 
In a study by Zolgharnein and Shahmoradi (2010), the statistical optimization in the removal of aqueous $\mathrm{Hg}^{2+}$ by Fraxinus tree leaves was investigated, and showed that nonlinear regression is the best way to model equilibrium data. Screening designs identify factors or key variables that influence a given response, which assist in narrowing down the parameters to save time and cost to the experimental design; a $2^{3}$ full factorial design was used to identify significant factors and interactions. In a batch-mode bio-sorption process with a fixed contact time of 30 minutes, the $\mathrm{pH}$, initial $\mathrm{Hg}^{2+}$ concentration, and sorbent mass were considered major factors, with a $95 \%$ confidence level. A multiple regression model was derived by using a response surface methodology (central composite design) and adequacy checked by diagnostic tests (i.e., analysis of variance (ANOVA)). In the 'presence of interaction', the factors may affect the response interactively and not in an independent way; their combined effect may be greater or less than that expected for the straight addition of the overall effects on removal. Zolgharnein and Shahmoradi (2010) state that the initial concentration had the greatest affect, such that increasing its level from low to high decreases the removal. In the analysis of the interaction between concentration and mass, increasing the level of mass has a less pronounced effect when the concentration is at a low level than when at a high level. The study by Zolgharnein and Shahmoradi (2010) determined the optimum parameter conditions under the testing conditions. A recommendation for future analysis would be to approach this statistical experimental design of multivariate linear regression, with an emphasis on field data, and apply it to the optimization of the continuous-mode of the treatment system prototype.

The novelty of the treatment system prototype presented in Chapter $\mathbf{7}$ of this study is greatly attributed to the unique controlled sampling technique, with a design that enables the control of the flow distribution in the system by various modes of operation. The current design incorporates a unique stock flow feed in a timed 'circulate-pulse' method, with discrete threeway solenoid valves that divert $100 \%$ of the flow. The flow is controlled in the first column by the pump at the system inlet, while the flow in the second column is controlled by the "circulatepulse' average sampling between Mode-II and Mode-III. The three-way solenoid valves and adjustable distribution timer would enable future investigations of variable system inlet flow rates and sample configurations (i.e., collection volume, automatic timer diversion settings) in 
Mode-III of operation. Future considerations to tune the nominal average flow would be to investigate modulating valves, which would divide the flow to a desired percentage, making the system a truly continuous hydraulic flow regime.

The complete removal of lead and iron is of significance to the practicality of the prototype. Under the testing conditions, this presents the opportunity to isolate a particular set(s) of HMIs for recovery, and reprocessing the effluent for use in pertinent, riveted industry. Based on the HMI removal selectivity trends reported in the current design under the study testing conditions, a third column would be ideal to refine the treatment efficiency. The particle size $\left(d_{p}\right)$ and column inlet flow rate may be manipulated; coarsest and quickest for the first column to target $\mathrm{Pb}^{2+}$ and $\mathrm{Fe}^{3+}$, finer and slower for the second column to target $\mathrm{Cu}^{2+}$ and $\mathrm{Zn}^{2+}$, and finally the finest size and slowest rate for the third column to target $\mathrm{Ni}^{2+}$; keeping in mind pump head and back pressure effects in the refinement of these parameter settings. Of the various multi-phase reactor configurations, fixed-bed reactors (FBRs) provide little axial dispersion, operating very similar to plug flow, while slurry bubble columns (SBCs) demonstrate excellent heat and mass transfer characteristics (Kantarci et al., 2005) and provide short residence time for sorption removal (Cui et al., 2006). The distributed gas in a SBC generates bubbles, which entrain the solution and causes a greater overall upflow in the column (Kantarci et al., 2005). This prompts the idea for future system design to create a FBR-SBC hybrid system. Prospective configurations may also include two columns connected in parallel to replace a single column, facilitating disconnection and replacement while maintaining the system circulation in Mode-II; all with consideration to the column aspect ratio to zeolite particle size. A significant improvement to this study would be to extend the 3-hour contact time, in order to associate the equilibrium state capacity experimentally and theoretically, as well as evaluate various operative conditions to generate further insight into associated mechanisms in the rate of uptake by natural zeolite. With further optimization, this prototype is a major contribution to the scientific community, and is a worthwhile pursuit being a platform for future FBR design in the treatment of industrial wastewater effluents.

This research project has explored the removal capacity of natural zeolite for heavy metals, and has provided greater insight for management and remediation. With future optimization, the innovative prototype is considered a transferrable technology in the treatment of industrial 
wastewater effluents. Evidently, this proposed technology benefits both the industry and Canada, with an overall improved environmental awareness. Metal mining and industrial processing must consider the environmental sustainability and overall impact in regards to water use, reuse, and disposal. Holistic water management and site water balance are critical for existing and new industry ventures. A shift in thinking is necessary towards a more systematic and strategic approach towards a balance among risk and conservatism (Nicholson, 2015), with effective industry operation, waste management (Zinck et al., 2005), and water quality quantification (Nicholson, 2015). Hydrologic and geo-chemical characterization, mitigation measures, as well as time are all considered major modes of prediction failure (Nicholson, 2015). With this perception, the major contributions of this research will lead to a greater knowledge-base of the behaviour and mechanisms within the interaction of zeolite and heavy metallic ions, and the overall sorption removal efficiency. The critical conclusions reached by this research may evolve to enable the industry to recognize how to best employ the natural mineral zeolite in its current treatment methodology, as part of the system design protocol for industrial wastewater in the future. 


\section{APPENDIX A. Experimental Methodology Procedures}

\section{A.1. Laboratory Health and Safety}

The protocols followed and procedures conducted in the laboratory are of significance to the scientific community. This subsection is provided to establish the necessary foundation for best practice principles in the continuation and future optimization of this research project.

In accordance with Ryerson University and the Department of Environmental Health and Safety (EHS), all personnel involved underwent training with respect to handling (preservation, transportation, receiving), experimental testing and analysis. At the time of experimental design, correspondence took place with the Faculty of Engineering and Architectural Science (FEAS) EHS advisor Mr. Eric Ambroise and the Radiation-Chemical-Biosafety Officer Ms. Valerie Phelan. All chemicals (i.e., metal nitrate salts, nitric acid) were thoroughly classified (as per their respective compositions and MSDS information), to establish a detailed protocol in the laboratory; in accordance with the Ryerson University Impact Assessment procedure.

Among the heavy metals proposed, it is important to note that lead is classified as a 'designated substance' with carcinogenic risks. With a Time-Weighted Average (TWA) 0.05 $\mathrm{mg} / \mathrm{m}^{3}$, all manipulations must be conducted within a fume-hood to ensure that the regulated exposure limit is met. Consequently, a fume-hood inspection was established for air velocity levels and nitrate salt particulate (aerosol) movement. Ms. Phelan scheduled calibration, and verified that the rate read at $120 \mathrm{ft} / \mathrm{min}$. A smoke test showed that all the air currents moved into the fume-hood and was consumed by the fan; its condition was confirmed as satisfactory to move forward with all experimental work. With regards to the nitrate salt particulate movement, Mr. Ambroise conducted a dust mask fit test with a $3 \mathrm{M}^{\mathrm{TM}}$ (8210) N95 NIOSH certified disposable respirator. With these masks, Ms. Phelan conducted the aerosol test for specifically lead nitrate, and the meter did not detect any measurable aerosols with the demonstrated manipulations of the salt. With the solutions under containment in the fume-hood, it was determined that there was no need for masks. A baseline blood test was made for lead $\left(\mathrm{Pb}^{2+}\right)$ levels by the Ph.D. Candidate, and returned to their general physician following all experimental exposure, and ensured that the lead containment met health and safety requirements. 
In addition to metal nitrate salt handling, concentrated nitric acid (CAS No. 7697-37-2) is a critical aspect to the proposed research. The acid was used in routine cleaning, $\mathrm{pH}$ control and sample preservation. Consequently, chemical-resistant, impervious material complying with approved standards was required for specific protection; including neoprene gloves to protect the hands and arms, a face shield to avoid exposure to liquid splashes, mists or dusts, as well as a body apron when handling the concentrated nitric acid. All safety gear specifications are presented in Table A.1.

The following points should also be highlighted in the extensive efforts made by the Ph.D. Candidate to ensure a safe research environment:

1. Proper management of all materials and equipment is executed during every experimental session, both in MON412 and the ANALEST centre, with the utmost diligence.

2. The lab bench stations are maintained in a clean and orderly manner.

3. Only clean absorbent lab paper is used for labware and experimental sequences, while soiled dry paper is kept and reused for the lab floor.

4. During the handling of the acid bath:

a. The nitrile examination gloves are covered with the neoprene gloves.

b. The lab coat sleeves are tucked into the neoprene gloves.

c. The fume-hood window and face shield are used at all times.

5. All residual zeolite sorbent material and HMI sorbate solution, as well as concentrated nitric acid are routinely disposed into a jerrican and stored under the MON412 fume-hood.

It should be noted that the training provided to all personal involved are at levels which are sufficiently elaborate and commensurate with the level of their respected programs of study. The Ph.D. Candidate has administered the research project in its entirety, and delegated the undergraduate students involved primarily in labware cleaning, where deemed appropriate by the technician of the Environmental Engineering Laboratory. 


\section{A.2. Materials and Equipment - Inventory and Specifications}

The record of all materials and equipment are presented Table A.1, followed by their specifications in Table A.2 and Table A.3, respectively.

Table A.1 Inventory of Consumable Materials and Related Equipment

\begin{tabular}{|c|c|c|c|c|}
\hline \multicolumn{2}{|r|}{ Item } & Supplier & CAT No. & Description \\
\hline \multicolumn{2}{|c|}{$\begin{array}{l}\text { Natural } \\
\text { Zeolite }\end{array}$} & $\begin{array}{l}\text { Bear River } \\
\text { Zeolite }\end{array}$ & Z 2-gal & $\begin{array}{l}2 \text { gallon bucket of }-8+40 \text { mesh BRZ } \\
2 \text { gallon bucket of }-14+40 \text { mesh BRZ } \\
2 \text { gallon bucket of } 30 \times 60 \text { mesh BRZ } \\
5 \text { gallon bucket of }-14+40 \text { mesh BRZ }\end{array}$ \\
\hline \multicolumn{2}{|c|}{$\begin{array}{l}\text { Analytical Grade } \\
\text { Nitrate Salts }\end{array}$} & $\begin{array}{l}\text { Sigma- } \\
\text { Aldrich }\end{array}$ & $\begin{array}{l}61194-500 G \\
216828-500 G \\
72252-500 G \\
228621-500 G \\
228737-500 G\end{array}$ & $\begin{array}{l}\text { puriss. p.a., } 99-104 \% \\
98+\%-A C S \text { reagent, } \geq 98 \% \\
\text { puriss. p.a., } \geq 98.5 \% \\
99+\% \text {-ACS reagent, } \geq 99.0 \% \\
\text { reagent grade, } 98 \%\end{array}$ \\
\hline \multicolumn{2}{|c|}{$\begin{array}{c}\text { ICP } \\
\text { Standard }\end{array}$} & $\mathrm{SCP}$ & $\begin{array}{l}140-102-042 \\
140-102-045\end{array}$ & $\begin{array}{l}\text { Multi-Element - Quality Ctrl.Std. } 4250 \mathrm{ml} \\
\text { Multi-Element - Quality Ctrl.Std. } 4500 \mathrm{ml}\end{array}$ \\
\hline \multicolumn{2}{|c|}{ Nitric Acid } & VWR & CANX0407-2 & NITRIC ACID OMNITRACE,2.5L (EM-NX0407-2) \\
\hline \multicolumn{2}{|c|}{$\begin{array}{c}\text { Standard } \\
\text { Mesh Sieves }\end{array}$} & $\begin{array}{c}\text { Fisher } \\
\text { Scientific } \\
\end{array}$ & $\begin{array}{l}04881 \mathrm{G} \\
04881 \mathrm{~L} \\
57334-104 \\
57334-106 \\
57334-108 \\
57334-112 \\
57334-114 \\
57334-116 \\
57334-118 \\
57334-120 \\
\end{array}$ & $\begin{array}{l}\text { Fisherbrand Sieve Brass 8" } 9 \text { Mesh US \#10 } \\
\text { Fisherbrand Sieve Brass 8" 16 Mesh US \#18 } \\
\text { VWR SIEVE FULL 8IN BRASS \#12 } \\
\text { VWR SIEVE FULL 8IN BRASS \#14 } \\
\text { VWR SIEVE FULL 8IN BRASS \#16 } \\
\text { VWR SIEVE FULL 8IN BRASS \#20 } \\
\text { VWR SIEVE FULL 8IN BRASS \#25 } \\
\text { VWR SIEVE FULL 8IN BRASS \#30 } \\
\text { VWR SIEVE FULL 8IN BRASS \#35 } \\
\text { VWR SIEVE FULL 8IN BRASS \#40 }\end{array}$ \\
\hline \multirow[t]{2}{*}{ Labware } & Glassware & $\begin{array}{l}\text { Fisher } \\
\text { Scientific }\end{array}$ & $\begin{array}{l}10206 \mathrm{H} \\
10206 \mathrm{G} \\
10206 \mathrm{D} \\
08555 \mathrm{D} \\
08555 \mathrm{G}\end{array}$ & $\begin{array}{l}\text { Corning-Pyrex Flask Volumetric 1000mL } \\
\text { Corning-Pyrex Flask VOL 500ML CS/12 } \\
\text { Corning-Pyrex Flask VOL 100ML PK/6 } \\
\text { Cylinder White Line 100ML } \\
\text { Cylinder White Line 1000ML }\end{array}$ \\
\hline & \begin{tabular}{|c|} 
Sample \\
Bottles \\
(Polypropylene) \\
\end{tabular} & $\begin{array}{l}\text { Fisher } \\
\text { Scientific }\end{array}$ & $\begin{array}{l}\text { 02893AA } \\
02893 \mathrm{BB} \\
02893 \mathrm{~A}\end{array}$ & $\begin{array}{l}\text { Nalgene Bottle Wide Mouth PP 1oz ( } 30 \mathrm{~mL}) \text { PK/12 } \\
\text { BOTTLE WM PP 2OZ 12/PK } \\
\text { Nalgene Bottle Wide Mouth PP 4oz (125 mL) PK/12 }\end{array}$ \\
\hline \multicolumn{2}{|c|}{$\begin{array}{c}\text { ICP } \\
\text { Sample Tubes }\end{array}$} & $\begin{array}{c}\text { Fisher } \\
\text { Scientific }\end{array}$ & $\begin{array}{l}0553859 \mathrm{~B} \\
0553860\end{array}$ & $\begin{array}{l}\text { Corning-TB 15ML CLR PP FLT (Corning) CS/500 } \\
\text { Corning-TUBE 50ML W/RIMSEAL CAP (Corning) CS/500 }\end{array}$ \\
\hline \multicolumn{2}{|c|}{ Filter } & VWR & CA97005-232 & Syringe Filter-FILTER 30MM RC 0.45UM PK100 \\
\hline \multicolumn{2}{|c|}{ Syringe } & $\begin{array}{c}\text { Fisher } \\
\text { Scientific }\end{array}$ & $\begin{array}{l}337730 \\
2137752\end{array}$ & SYRINGE DSP PLST LL 20ML PK100 \\
\hline
\end{tabular}


Table A.1 Inventory of Consumable Materials and Related Equipment (continued)

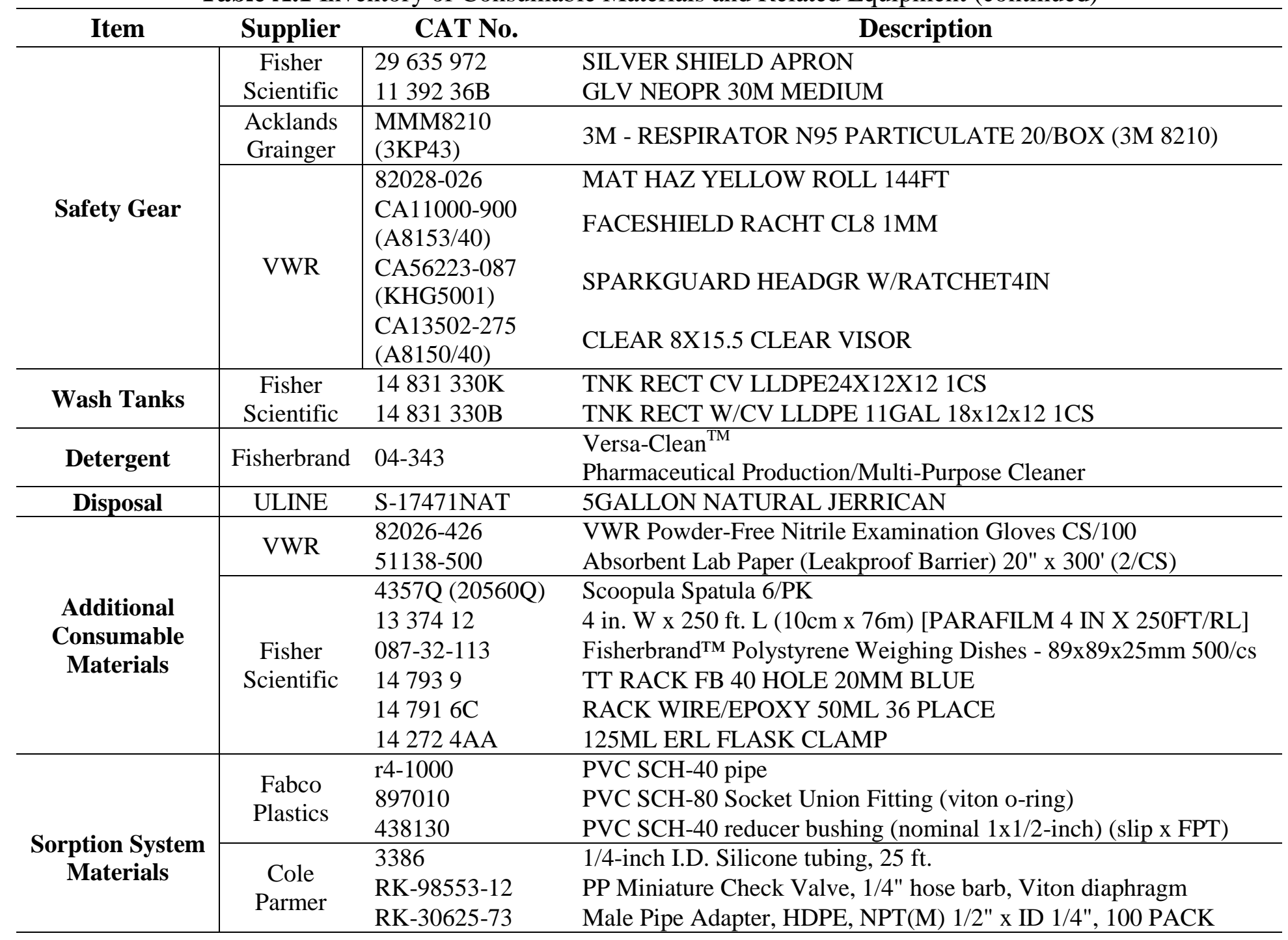


Table A.2 Specifications of Consumable Materials

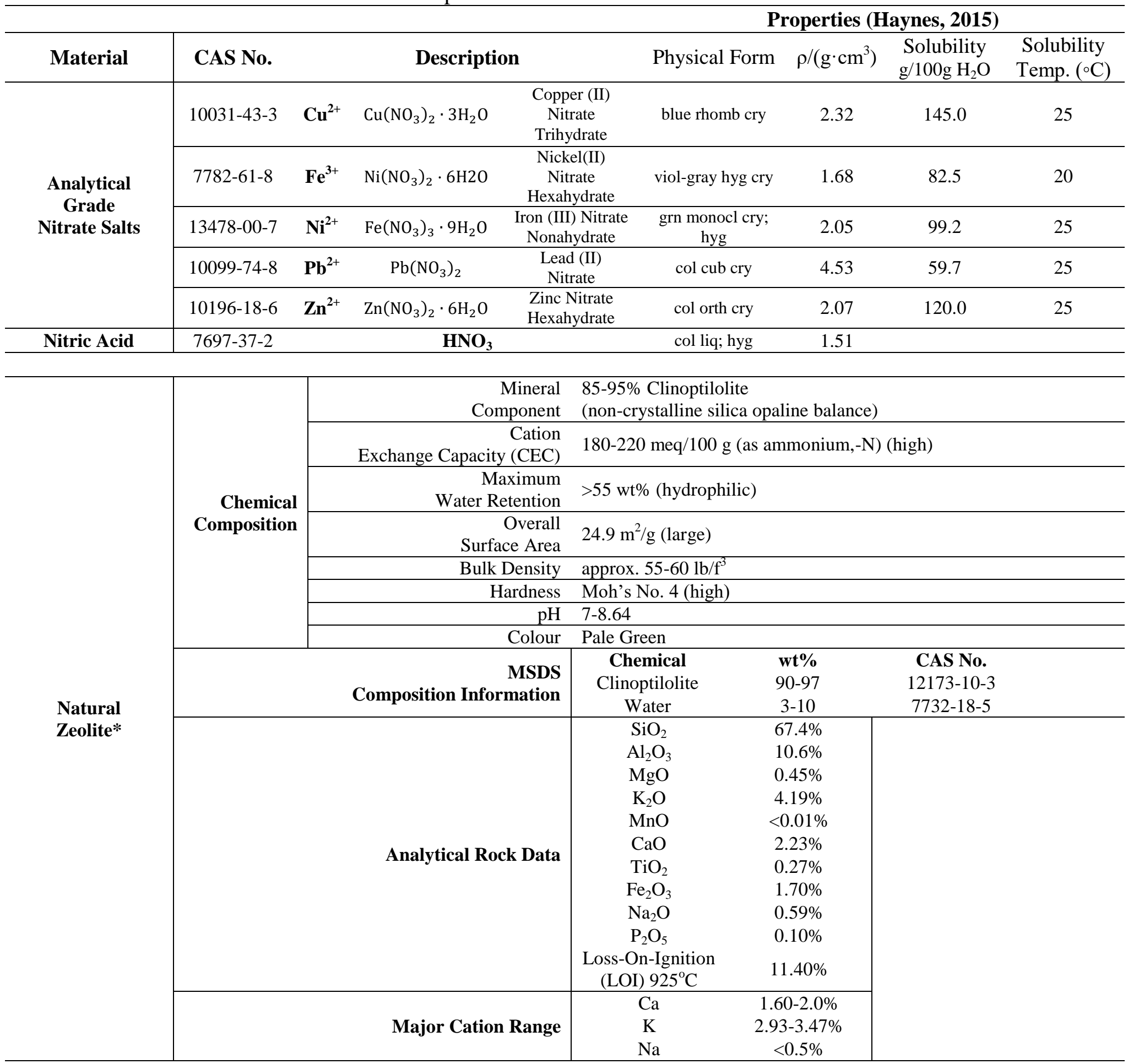

*(Bear River Zeolites, 2012; 2017) 
Table A.3 Specifications of Related Equipment

\begin{tabular}{|c|c|}
\hline Equipment & Specifications \\
\hline Scanning Electron & 6380LV (EDS, EBSD, 3D) \\
\hline Microscopy (SEM) & JEOL, USA \\
\hline \multirow{4}{*}{$\begin{array}{l}\text { Inductively Coupled Plasma - } \\
\text { Atomic Emission Spectroscopy } \\
\text { (ICP-AES) }\end{array}$} & Optima 7300 DV \\
\hline & Part No. N0770796; Serial No. 077C8071802 \\
\hline & Firmware Version 1.0.1.0079 \\
\hline & Perkin Elmer Inc., Waltham, MA, USA \\
\hline \multirow{2}{*}{$\begin{array}{c}\text { Mechanical } \\
\text { Sieve }\end{array}$} & Model No. Humboldt H4330 \\
\hline & CAT No. G118-H-4330 \\
\hline \multirow{3}{*}{ Orbital Shaker } & MaxQ ${ }^{\text {TM }} 4450$ Benchtop Orbital Shaker \\
\hline & CAT No. $11-675-202$ \\
\hline & ThermoFisher Scientific \\
\hline \multirow{3}{*}{ pH Meter } & accumet Basic AB15 pH Meter \\
\hline & CAT No. 13636 AB15 \\
\hline & Fisher Scientific \\
\hline \multirow{4}{*}{ Pipette } & Finnpipette \\
\hline & $0.1-1 \mathrm{~mL} ; 2-10 \mathrm{~mL}$ \\
\hline & CAT No. $21-377-821 ; 21-377-824$ \\
\hline & Thermo Electron Corporation \\
\hline \multirow{4}{*}{ Muffle Furnace } & NEY M-525 SII \\
\hline & Serial No. AKN 9403-108 \\
\hline & $120 \mathrm{~V} ; 50 / 60 \mathrm{~Hz} ; 12.5 \mathrm{~A} ; 1500 \mathrm{~W}$ \\
\hline & Barkmeyer Division, USA \\
\hline \multirow{4}{*}{ Oven } & Isotemp® Oven Model 630G \\
\hline & Serial No. 30300047 ; CAT No. 13-246-630G \\
\hline & $115 \mathrm{~V} ; 6.5 \mathrm{~A} ; 60 \mathrm{~Hz}$ \\
\hline & Fisher Scientific, USA \\
\hline \multirow{2}{*}{ Fume-hood } & CIF; Con-Test Calibration \\
\hline & Sash Height: 18; Velocity: $175 \mathrm{fpm}$ \\
\hline \multirow{3}{*}{ Scale } & AL204 Analytical Scale \\
\hline & Fisher Scientific CAT No. 01910154 \\
\hline & Mettler Toledo \\
\hline \multirow{3}{*}{ DDW } & Direct-Q ${ }^{\circledR}$ Water Purification System \\
\hline & Fisher Scientific CAT No. ZRQSVPOUS \\
\hline & Millipore \\
\hline \multirow{4}{*}{$\begin{array}{c}\text { Diaphragm } \\
\text { Metering Pump }\end{array}$} & No. 950218125-C Plus \\
\hline & $\max$ 45-LPD 80-psi \\
\hline & $125-\mathrm{AC} 50 / 60-\mathrm{Hz}$ \\
\hline & PULSAtron, Punta Gorda, FL, USA \\
\hline \multirow{3}{*}{$\begin{array}{l}\text { Three-way } \\
\text { Solenoid Valve }\end{array}$} & No. 00457979 \\
\hline & 0124-C, 1/8-FKM-PP, NPT-1/4 \\
\hline & $\begin{array}{l}\max 145-\text { psi, } 24-\mathrm{V}, 60-\mathrm{Hz}, 8-\mathrm{W}, 38-\mathrm{mL} \\
\text { burkert, Ingelfingen, Germany }\end{array}$ \\
\hline
\end{tabular}


With respect to the specifications outlined in Table A.3, a view of the experimental set-up for SEM analysis is provided in Figure A.1. The ANALEST Centre facility employed for ICPAES analysis is captured in Figure A.2; providing the various components of the system as well as the software interface.

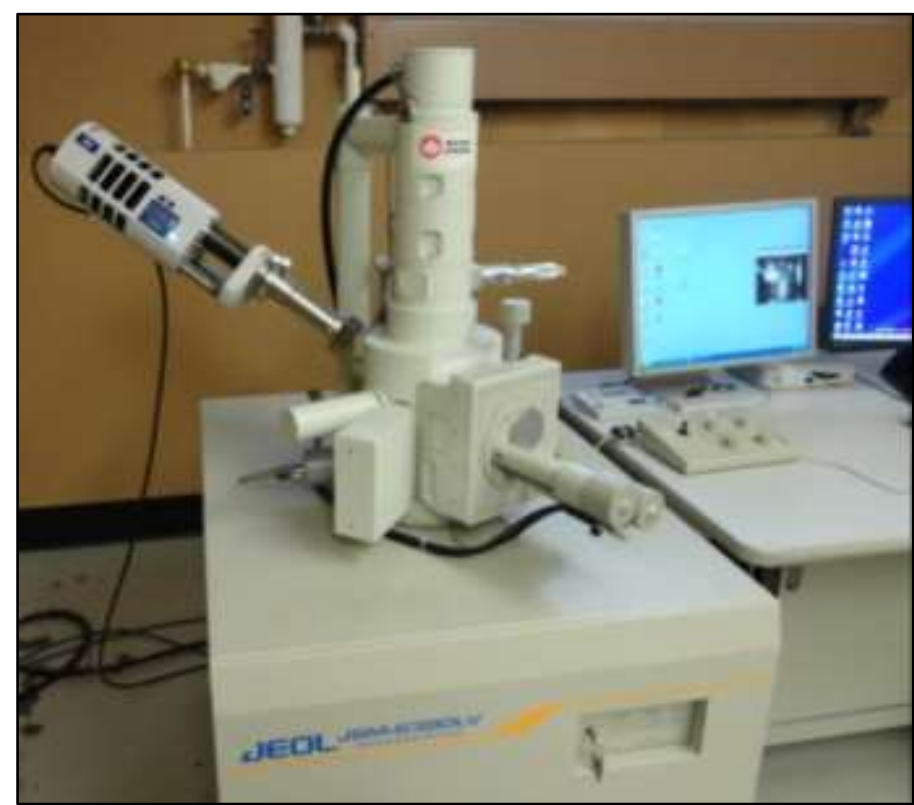

a. View of Microscope System

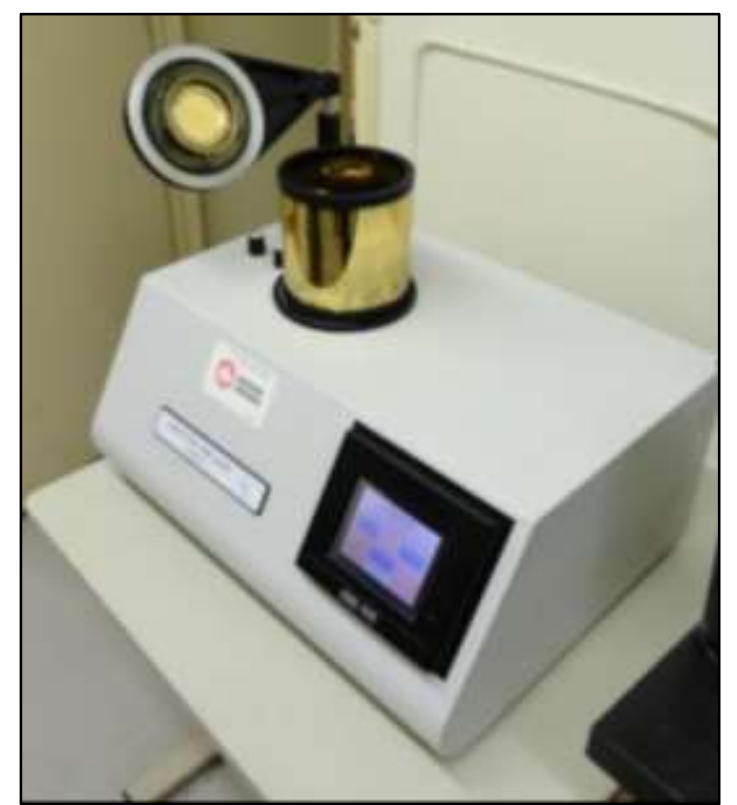

b. Preparation fo Conductive Layer (Sputter Coating Station)

Figure A.1 Experimental Set-up for SEM Analysis 


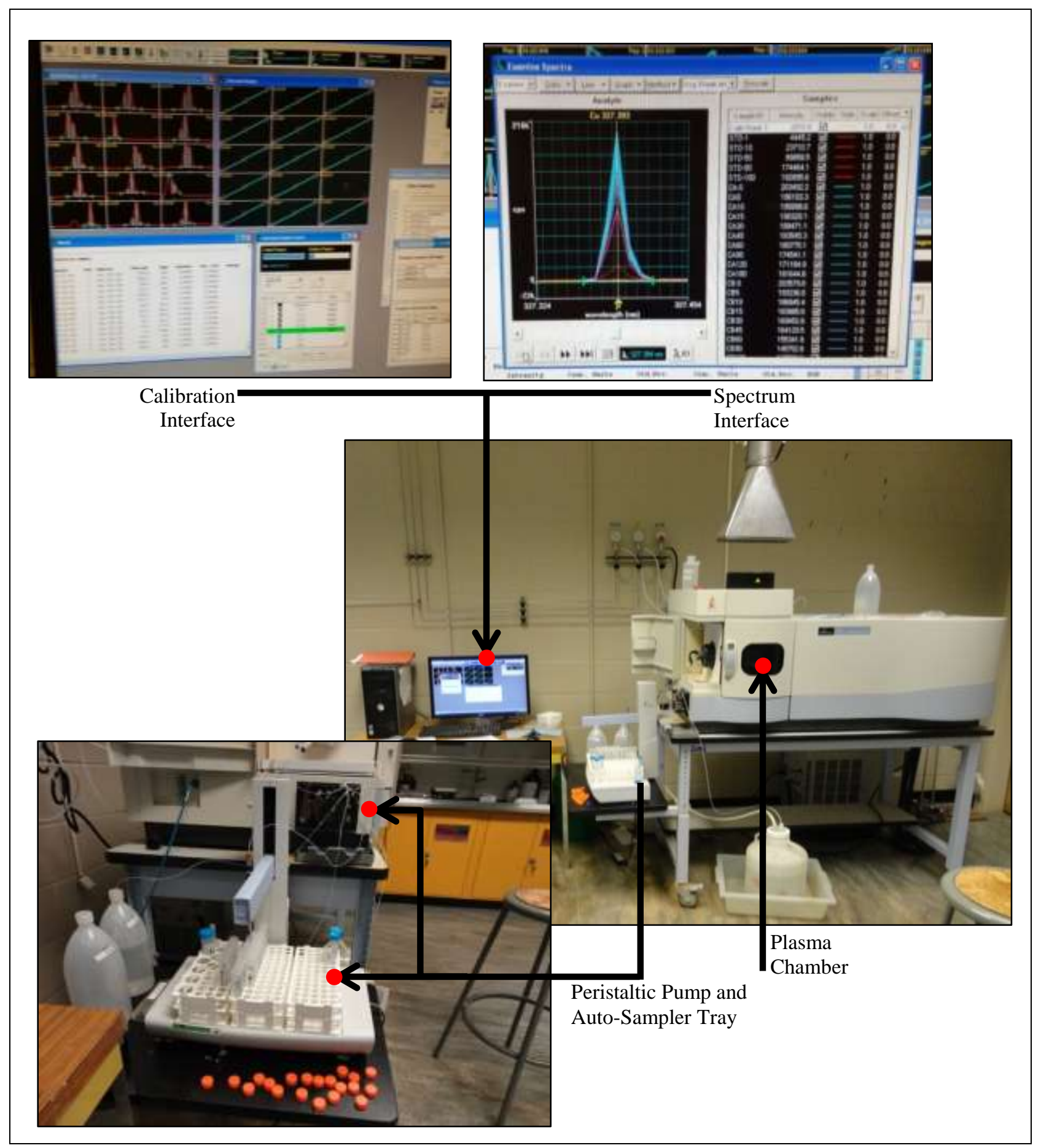

Figure A.2 Experimental Set-Up for ICP-AES Analysis 


\section{A.3. Environmental Engineering Laboratory Schedule}

Table A.4 is the schedule followed in the laboratory where all experimental work was conducted (MON412). During the Fall-2015 academic term, a bi-weekly schedule was followed to accommodate the undergraduate students at the time; the nitric acid bath was transferred from the fume-hood and out of reach on the floor for the safety of all personal in the shared space. During the Winter-2016 academic term, a 'two weeks on, one week off' schedule was followed, to provide time for lab management, routine data processing, analysis, and so on.

Table A.4 Laboratory Schedule

\begin{tabular}{|c|c|c|}
\hline \multirow[t]{2}{*}{ MONDAY } & $\mathrm{AM}$ & $\begin{array}{l}\text { - Collect DDW in carboys (4 L+) (per stock preparation) } \\
\circ \quad \text { Collect DDW-S (Blank) Sample } \\
\text { - Label Labware } \\
\circ \quad \text { Bottles }(1,2,4 \text { oz. }) \\
\circ \quad \text { Glassware }(2 \times 100 \mathrm{~mL}+4 \times 1-\mathrm{L} \text { Volumetric Flasks, } \\
4 \times 100 \mathrm{~mL} \text { Graduated Cylinders, } 4 \times \text { Beakers })\end{array}$ \\
\hline & $\mathrm{PM}$ & $\begin{array}{l}\text { - Measure Zeolite Mass to } 4 \text { oz. Bottle }(4 \times 9 \text { Samples }) \\
\text { - Prepare HMI Influent Stock (4× 1-L Sample Sets) } \\
\quad \circ \quad \text { Dilute HMI Nitrate Salts; Acidify (1.5mL per 1-L) } \\
\circ \quad \text { Refrigerator Storage } \\
\text { - Set-up experimental station on lab bench for Tuesday sequence }\end{array}$ \\
\hline \multirow{2}{*}{ TUESDAY } & $\mathrm{AM}$ & $\begin{array}{l}\text { - } \text { Collect DDW in carboys (per glassware clean) } \\
\text { - Set Incubator to } 400 \mathrm{rpm} \text { at } 22^{\circ} \mathrm{C}(08: 00 \mathrm{AM}) \\
\text { - Sample SET } 1+2(\mathrm{AM})(5 \text { to } 180 \mathrm{~min}) \\
\quad \mathrm{O} \text { Zeolite and HMI Stock }(100 \mathrm{~mL} \text { to } 4 \mathrm{oz} .) \\
\quad \text {. Filter and Refrigerator Storage }(1 \mathrm{oz} .) ; \mathrm{pH} \text { Measure }(4 \mathrm{oz} .) \\
\end{array}$ \\
\hline & PM & $\begin{array}{l}\text { - Set Incubator to } 400 \mathrm{rpm} \text { at } 22^{\circ} \mathrm{C}(12: 00 \mathrm{PM}) \\
\text { - } \\
\text { - } \text { Sample SET 3+4 (PM) } \\
\quad \circ \quad \text { Hazardous Waste Disposal; Tap Rinse + Soap Wash + Tap Rinse } \\
\quad \circ \quad \text { DDW Rinse }(1 \times)+\text { Air Dry } \\
\end{array}$ \\
\hline \multirow[b]{2}{*}{ WEDNESDAY } & $\mathrm{AM}$ & $\begin{array}{ll}\text { - } & \text { Acid Bath Labware } 1(8 \mathrm{AM}-24 \mathrm{hrs}+) \\
\text { - } & \text { Collect DDW in carboys (4 L+) (per dilution preparation) } \\
\text { - } & \text { Label ICP-AES Sample Tubes }\end{array}$ \\
\hline & PM & 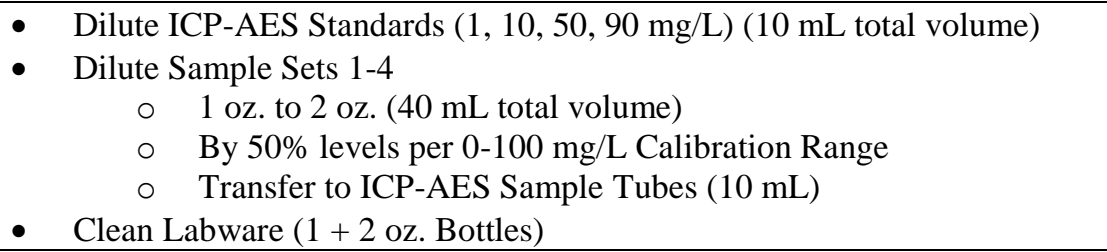 \\
\hline \multirow[b]{2}{*}{ THURSDAY } & $\mathrm{AM}$ & $\begin{array}{ll}\text { - } & \text { Remove Acid Bath Labware } 1 \text { and DDW }(3 \times) \text { Rinse } \\
\text { - } & \text { Acid Bath Labware } 2(10 \mathrm{AM}-24 \mathrm{hrs}+) \\
\end{array}$ \\
\hline & $\mathrm{PM}$ & $\begin{array}{l}\text { - } \text { Transport ICP-AES Sample Tubes (Portable Refrigerator) (12PM) } \\
\text { - University of Toronto ANALEST Centre } \\
\circ \quad \text { Plasma ON (1:00PM); Calibration (1:20-1:30PM) } \\
\circ \quad \text { Sample Analysis (1:30-2:45PM) } \\
\quad \text { ○ Data Reprocessing and Collection (2:45-3:00PM) } \\
\text { - Hazardous Waste Disposal ICP-AES Sample Tubes }\end{array}$ \\
\hline \multirow{2}{*}{ FRIDAY } & AM & - $\quad$ Remove Acid Bath Labware 2 + DDW (3x) Rinse \\
\hline & PM & - Digitize Lab Notes and Compile/Analyze ICP-AES Data \\
\hline
\end{tabular}




\section{A.4. Labware Cleaning Procedure}

1. Create soap mixture with by filling soap bottle with $20: 1$ ratio; $300 \mathrm{~mL} \mathrm{DDW}+25 \mathrm{~mL}$ soap $+200 \mathrm{~mL}$ DDW.

2. Dispose of spent zeolite sorbent material and HMI sorbate solution from bottle(s).

3. Dispose of spent residual HMI sorbate solution from glassware.

4. Rinse labware once with warm tap water and place on clean absorbent lab paper.

5. Fill labware with soap mixture and shake well.

6. Rinse labware with warm tap water until all of the soap mixture disappears; approximately nine (9) rinse cycles.

7. Dry labware on clean absorbent lab paper.

8. Rinse labware once (1 $\times$ ) with DDW and dry on clean absorbent lab paper overnight.

9. Soak labware (submerge bottles and fill-parafilm cap glassware) in 1+1 acid bath overnight (24 hrs).

10. Remove labware from acid bath, shaking off any residual acid solution.

11. Rinse labware three times with DDW water.

12. Dry labware on clean absorbent lab paper.

*Note - Conventional fume-hoods significantly contribute to contamination. Consequently, all vessels are kept covered from incoming air. 


\section{A.5. HMI Influent Stock Preparation}

Table A.5 provides the calculations based on a total HMI component system concentration of $0.01 \mathrm{~N}(10 \mathrm{meq} / \mathrm{L})$ of the respective analytical grade nitrate salts (Inglezakis et al., 2002; Inglezakis et al., 2003; Inglezakis et al., 2004; Stylianou et al., 2007a). The required nitrate salt mass that corresponds to the HMI concentration (represented in $\mathrm{mg} / \mathrm{L}$ ) are used for the dilution preparations required for elemental analysis (discussed in Appendix A.9).

Table A.5 Influent Concentration Computation

\begin{tabular}{|c|c|c|c|c|c|c|c|}
\hline \multirow{2}{*}{ HMI } & \multirow{2}{*}{$\begin{array}{c}\text { Nitrate Salt } \\
\text { CAS No. }\end{array}$} & \multicolumn{6}{|c|}{ Molecular Weight [MW] (g/mol) } \\
\hline & & \multicolumn{2}{|c|}{ Compound HMI } & & & & \\
\hline $\mathrm{Cu}^{2+}$ & $10031-43-3$ & 241.602 & 63.546 & & & & \\
\hline $\mathrm{Fe}^{3+}$ & $7782-61-8$ & 403.997 & 55.845 & & & & \\
\hline $\mathrm{Ni}^{2+}$ & $13478-00-7$ & 290.794 & 58.693 & & & & \\
\hline $\mathbf{P b}^{2+}$ & $10099-74-8$ & 331.200 & 207.200 & & & & \\
\hline $\mathrm{Zn}^{2+}$ & 10196-18-6 & 297.510 & 65.380 & & & & \\
\hline \multirow[b]{2}{*}{ HMI } & \multicolumn{5}{|c|}{$\begin{array}{c}\text { Conversation Steps } \\
\end{array}$} & \multicolumn{2}{|c|}{ Concentration $(\mathrm{mg} / \mathrm{L})$} \\
\hline & $\% \mathrm{HMI}$ & $\begin{array}{l}\text { Normality } \\
{[N](e q / L)}\end{array}$ & $\begin{array}{c}\mathrm{K} \\
(\mathrm{eq} / \mathrm{mol})\end{array}$ & $\begin{array}{c}M(\mathrm{~mol} / \mathrm{L}) \\
\mathrm{M}=\mathrm{N} / \mathrm{K}\end{array}$ & $\begin{array}{c}\mathbf{M W} \cdot \mathbf{M} \\
(\mathrm{g} / \mathrm{L})\end{array}$ & Compound & HMI \\
\hline $\mathrm{Cu}^{2+}$ & 0.263 & \multirow{5}{*}{0.01} & 2 & 0.0050 & 1.2080 & 1208.010 & 317.730 \\
\hline $\mathrm{Fe}^{3+}$ & 0.138 & & 3 & 0.0033 & 1.3467 & 1346.657 & 186.150 \\
\hline $\mathrm{Ni}^{2+}$ & 0.202 & & 2 & 0.0050 & 1.4540 & 1453.970 & 293.465 \\
\hline $\mathbf{P b}^{2+}$ & 0.626 & & 2 & 0.0050 & 1.6560 & 1656.000 & 1036.000 \\
\hline $\mathbf{Z n}^{2+}$ & 0.220 & & 2 & 0.0050 & 1.4876 & 1487.550 & 326.900 \\
\hline
\end{tabular}

1. Collect deionized distilled water (DDW) $\left(18 \mathrm{~m} \Omega . \mathrm{cm} @ 25^{\circ} \mathrm{C}\right)$; approximately 4-L required for influent stock preparation contained in a $20 \mathrm{~L}$ carboy.

2. Weigh the analytical grade nitrate salt mass (measurements from Table A.5) using a scoopula spatula (labelled for each HMI and zeolite), weighing dish and balance (Figure A.3a and A.3b).

3. Dispense HMI salt into a 1-L volumetric flask, and rinse weighing dish with DDW to include total mass (Figure A.3c).

4. Dilute influent stock to 1-L (Figure A.3d).

5. Cover the 1-L volumetric flask with parafilm and invert slowly three times.

6. Remove the parafilm cover.

7. Measure $1.5 \mathrm{~mL}$ of concentrated nitric acid (Rice et al., 2012) and dispense into HMI solution (Figure A.3e).

8. Cover the 1-L volumetric flask with parafilm and invert slowly three times.

9. Store HMI influent stock in refrigerator overnight (Figure A.3f).

10. Collect DDW sample as blank for ICP-AES analysis of that experimental week. 


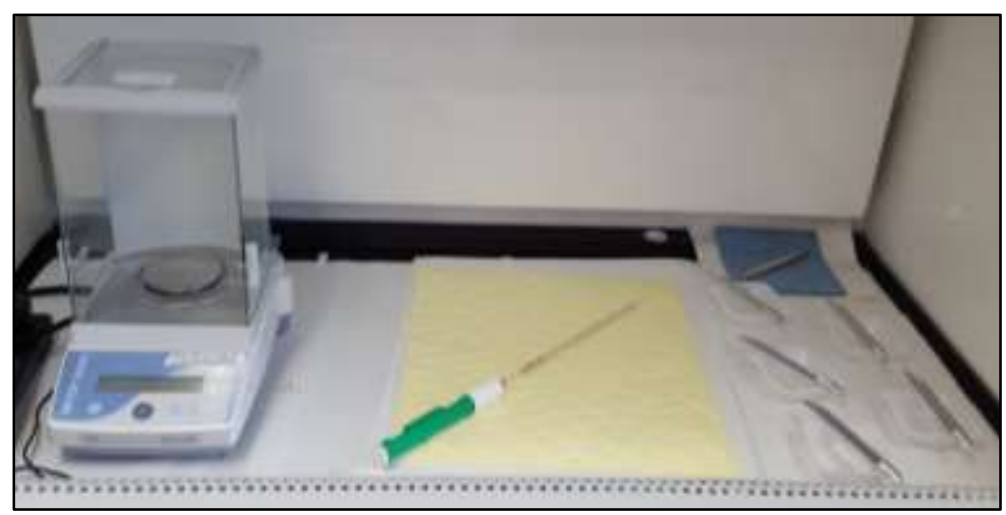

a. Set Aside Labware

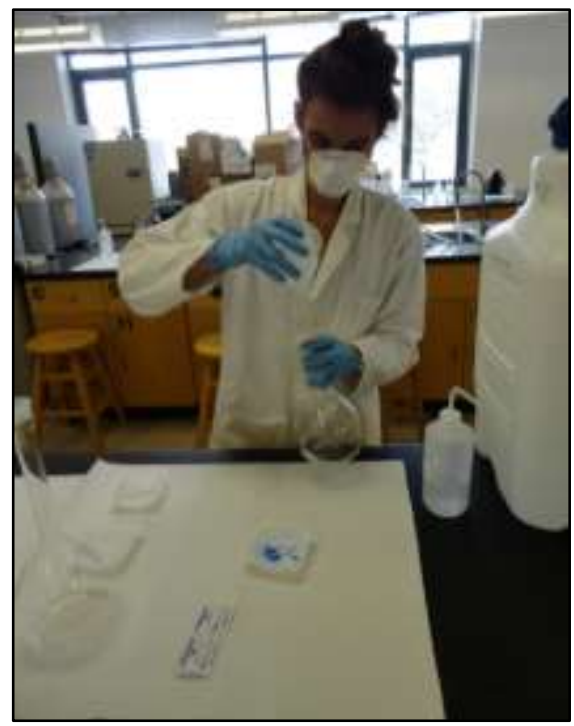

c. Dispense HMI Nitrate Salt into Flask

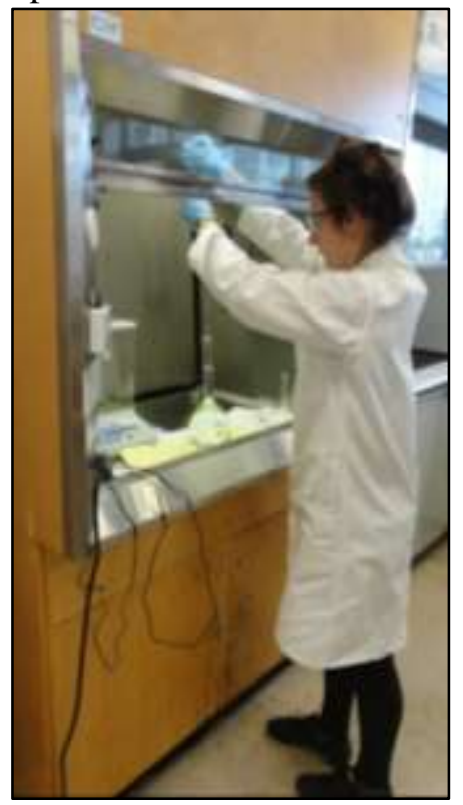

e. Acidify Stock

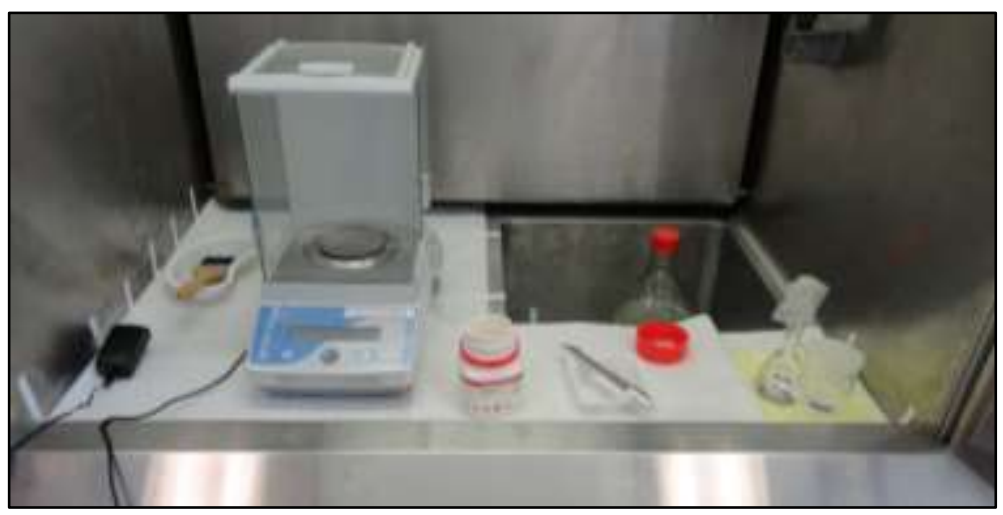

b. Weigh Nitrate Salts

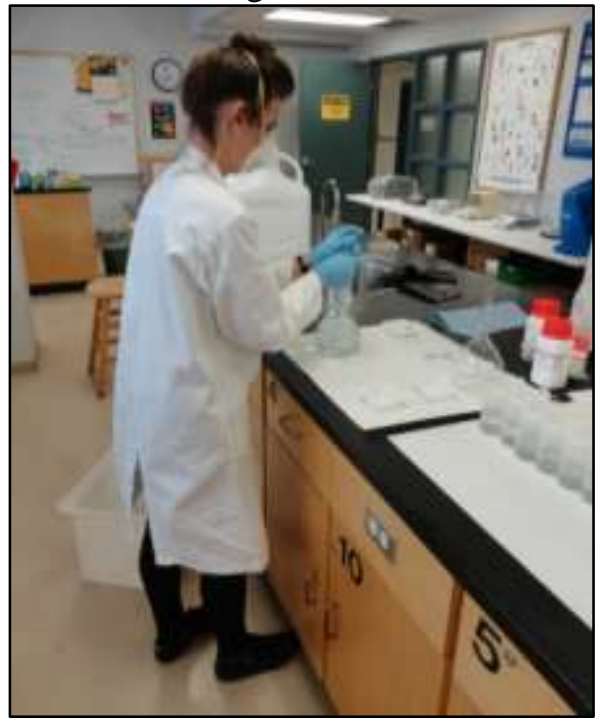

d. Dilute Stock to 1-L Volume

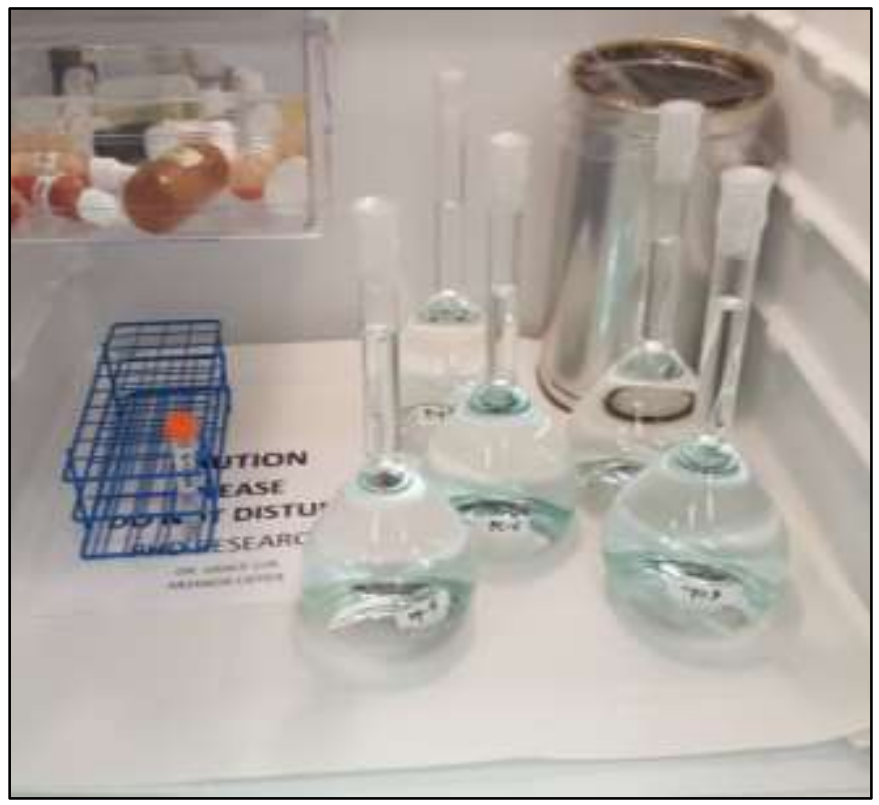

f. Store Prepared Influent Stock in Refrigerator

Figure A.3 HMI Influent Stock Preparation Procedure 


\section{A.6. Natural Zeolite Sample Preparation}

Preliminary analysis of the raw zeolite mineral supply was conducted in the geotechnical lab (KHN101), which involved a particle size range within the standard mesh fractions of $-8+40$, 14+40, and $-30+60$ from the supplier. As shown in Table A.6, testing of the particle size was conducted with standard mesh ranging from sieve sizes 10 to 40 . The results showed that the standard mesh range -14 (pass) +40 (retain) $(0.420-1.41 \mathrm{~mm})$ provided the most material once sieved along the available gradations. The zeolite particle size supply -14+40 is the basis of the parameters discussed in Chapter 4, divided into the sub-fractions of $A\left(d_{p, A}\right)(1.190-1.410 \mathrm{~mm})$, B $\left(\mathrm{d}_{\mathrm{p}, \mathrm{B}}\right)(0.707-0.841 \mathrm{~mm}), \mathrm{C}\left(\mathrm{d}_{\mathrm{p}, \mathrm{C}}\right)(0.420-0.595 \mathrm{~mm})$ and $\mathrm{D}\left(\mathrm{d}_{\mathrm{p}, \mathrm{D}}\right)(0.841-1.19 \mathrm{~mm})$. The particle size range $d_{p, D}$ is selected as the controlled parameter following the analysis of the particle size operation parameter.

1. Pre-sieve zeolite mass to the specified standard sieve gradations (particle sizes A, B, C and D of supply -14+40 as outlined in Table A.6);

2. Weigh approximately $300 \mathrm{~g}$ of raw (as-received) zeolite mass using a scoopula spatula (labelled for zeolite use), weighing dish and balance.

3. Transfer weighed zeolite to a shallow plastic container, and rinse with DDW water (approximately 1 inch of water above zeolite mass) until water solution is cleared of dust and debris; approximately nine (9) rinse cycles (Figure A.4a).

4. Place moist zeolite mass onto clean muffle furnace tray (Figure A.4b) and transfer to the oven (Figure A.4c).

5. Turn on oven and set to $80 \pm 3^{\circ} \mathrm{C}$ temperature.

6. Dry zeolite mass for $24 \mathrm{hrs}$.

7. Remove tray from the oven and allow zeolite mass to reach room temperature for $24 \mathrm{hrs}$.

8. Transfer zeolite mass from tray to a plastic bag for storage and forthcoming use. 
Table A.6 Particle Size Breakdown of Natural Zeolite Sorbent Supply

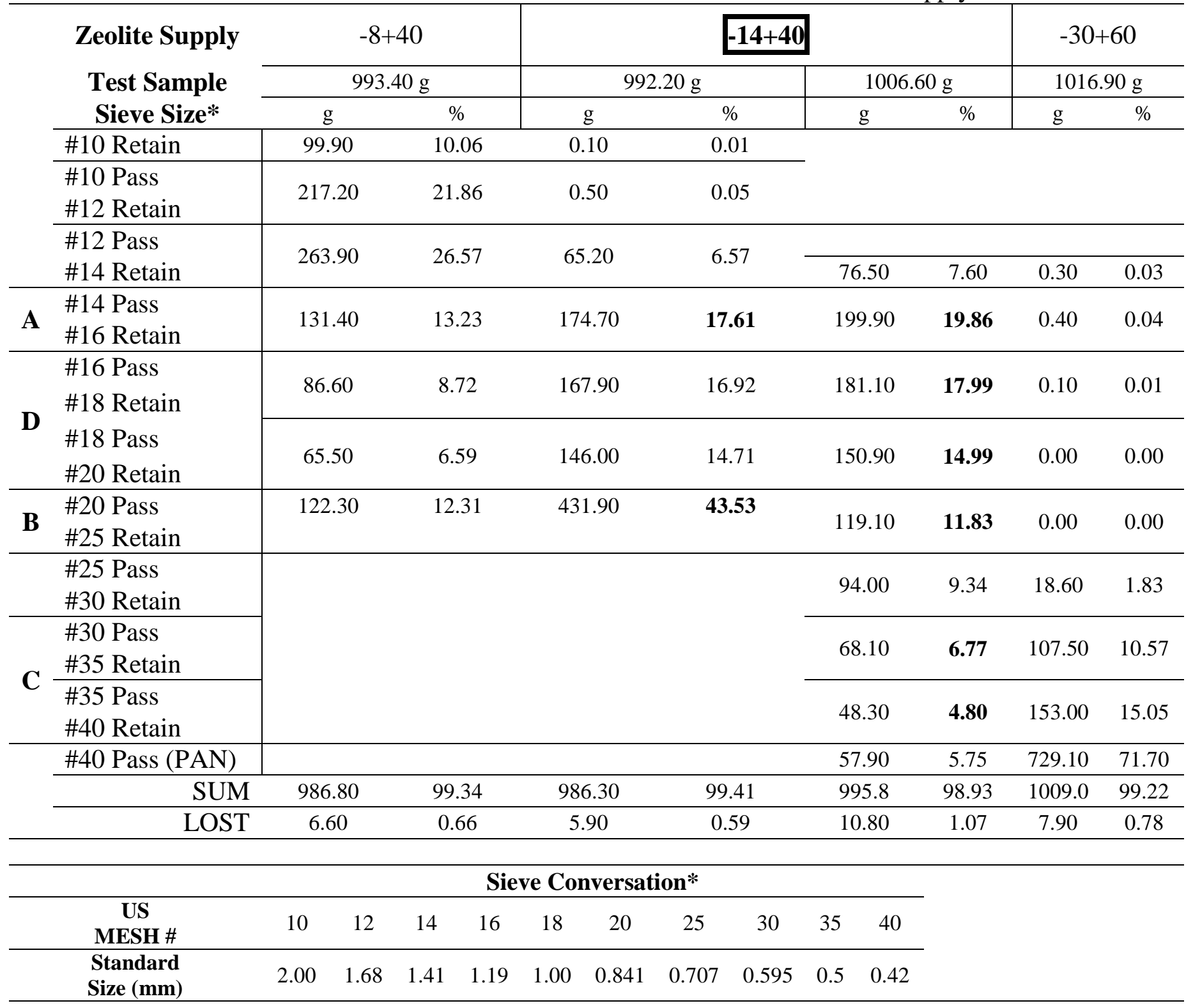




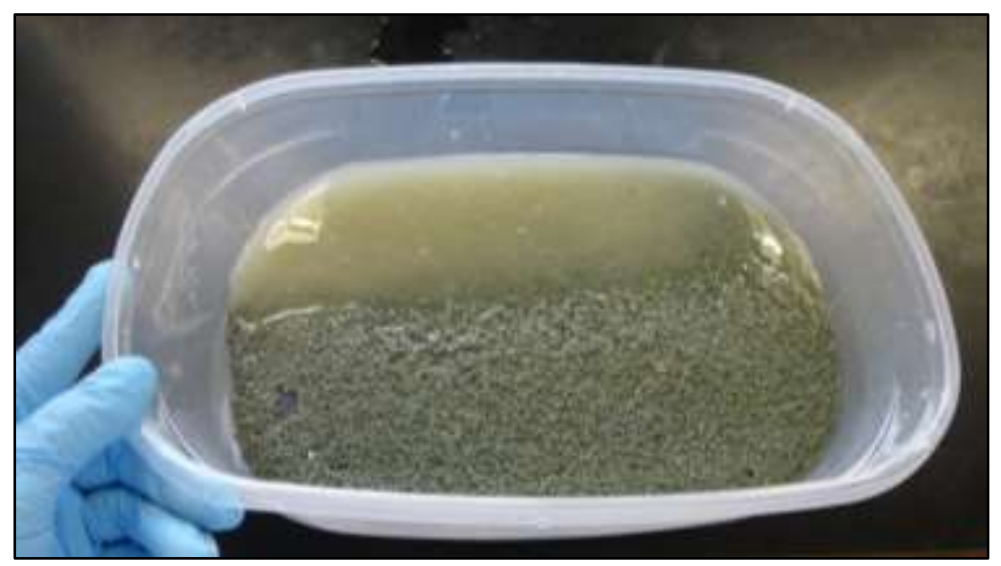

a. Remove Residual Debris from Raw Zeolite Sample

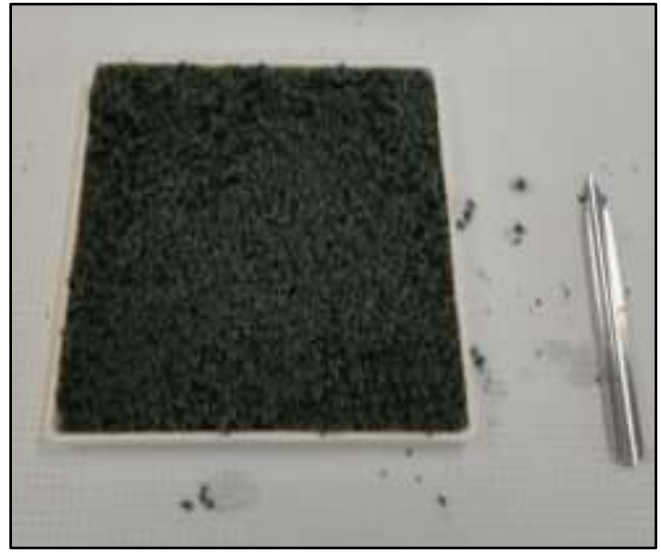

b. Place and Span out Zeolite onto Muffle Furnace Tray

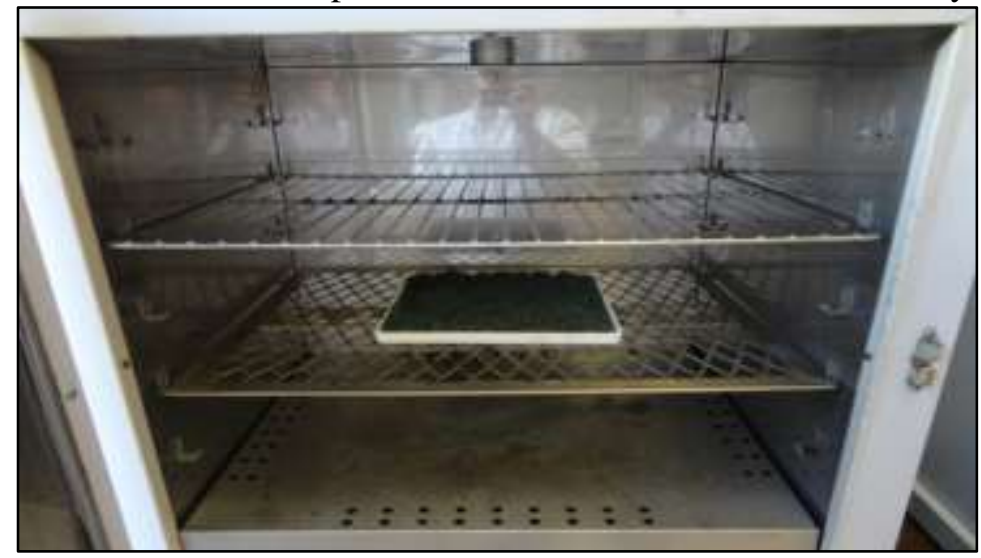

c. Remove Residual Moisture

Figure A.4 Natural Zeolite Sample Preparation - Cleaning Cycle Procedure 


\section{A.7. Hydrothermal Pre-Treatment Preparation}

1. The muffle furnace is pre-calibrated to ensure the mechanical dial system corresponds to the required heat pre-treatment level $\left(200^{\circ} \mathrm{C}, 400^{\circ} \mathrm{C}, 600^{\circ} \mathrm{C}\right)$ (Figure A.5a).

2. The cleaned natural zeolite is weighed using a scoopula spatula (labelled for zeolite use), weighing dish and balance; to the required mass of each heat level.

3. For each heat pre-treatment level, the zeolite mass is transferred to the muffle furnace tray and placed in the pre-heated muffle furnace (Figure A.5b).

4. Once the tray is inserted, the 1-hr treatment period starts once the desired heat level temperature is restored.

5. Remove the tray from the muffle furnace and allow zeolite mass to reach room temperature for $24 \mathrm{hrs}$.

6. Transfer zeolite mass from tray to a plastic bag for storage and forthcoming use.

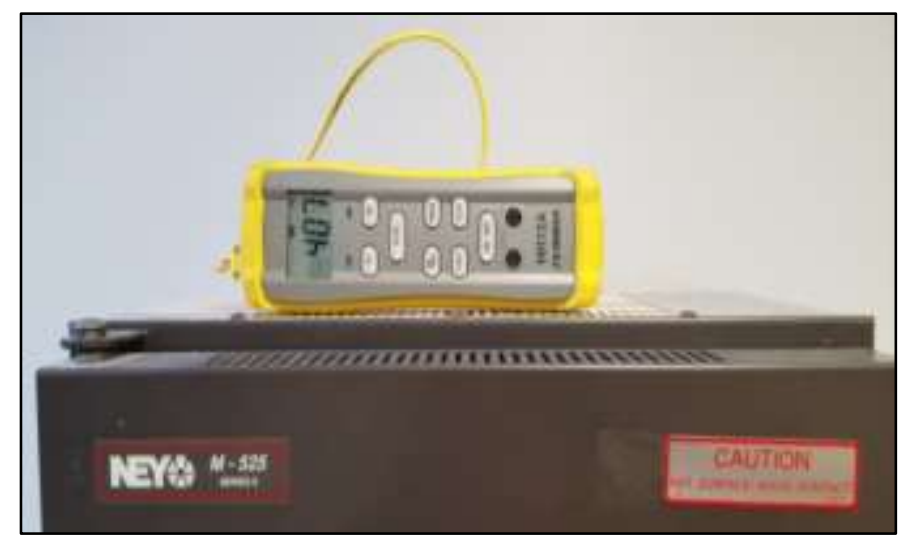

a. Verify Muffle Furnace Temperature Setting

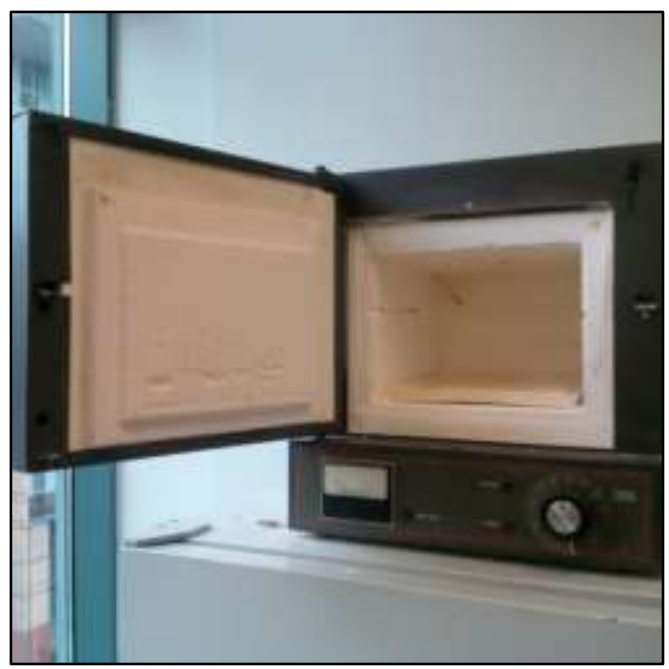

b. Place the Measured Zeolite Mass onto the Tray, then into the Muffle Furnace

Figure A.5 Hydrothermal Pre-Treatment of Zeolite Sample 


\section{A.8. Experimental Sequence Procedure}

- With reference to the lab sequence breakdown in Appendix A.3:

1. Remove AM SET 1+2 influent stock volumetric flasks from refrigerator (08:00).

2. Turn on orbital shaker and set to $400 \mathrm{rpm}$ at $22^{\circ} \mathrm{C}$ set temperature $(15 \mathrm{~min}$ prior to orbital shaker contact).

3. 'Rinse-out' ( $\sim 50 \mathrm{~mL})$ SET 1 and SET 2 100-mL graduated cylinders with each HMI influent stock.

4. As outlined in Table A.7, commence shaker-sample sequence; established to maximize space availability of shaker as specified with the order of sample bottles in (A) and out (B).

5. At each time-step, fill 4 oz. sample bottle (containing pre-weighed zeolite mass) with SET stock solution using $100-\mathrm{mL}$ graduate cylinder from the 1-L volumetric flask.

6. Place in bottle clamp position and press start button to initiate orbital shaker (Figure A.6a).

7. Position $1 \mathrm{oz}$. sample bottle and syringe-filter at the ready on bench station.

8. Remove bottle from orbital shaker.

9. Withdraw $25-\mathrm{mL}$ effluent sample with the syringe, twist-lock on the $0.45 \mu \mathrm{m}$ filter to syringe tip, and dispense the sample into the 1 oz. sample bottle (Figure A.6b).

10. Cap and store the $1 \mathrm{oz}$. sample bottle containing the effluent samples in the refrigerator overnight.

11. With the residual effluent contained in the 4 oz. sample bottle, collect a pH level measurement.

12. Dispose of spent zeolite sorbent material and HMI sorbate solution from bottle(s).

13. Dispose of spent residual HMI sorbate solution from glassware.

14. Repeat steps 1 to 13 for PM SET 3+4 (12:00). 
Table A.7 Orbital Shaker Sample Bottle Contact Sequence

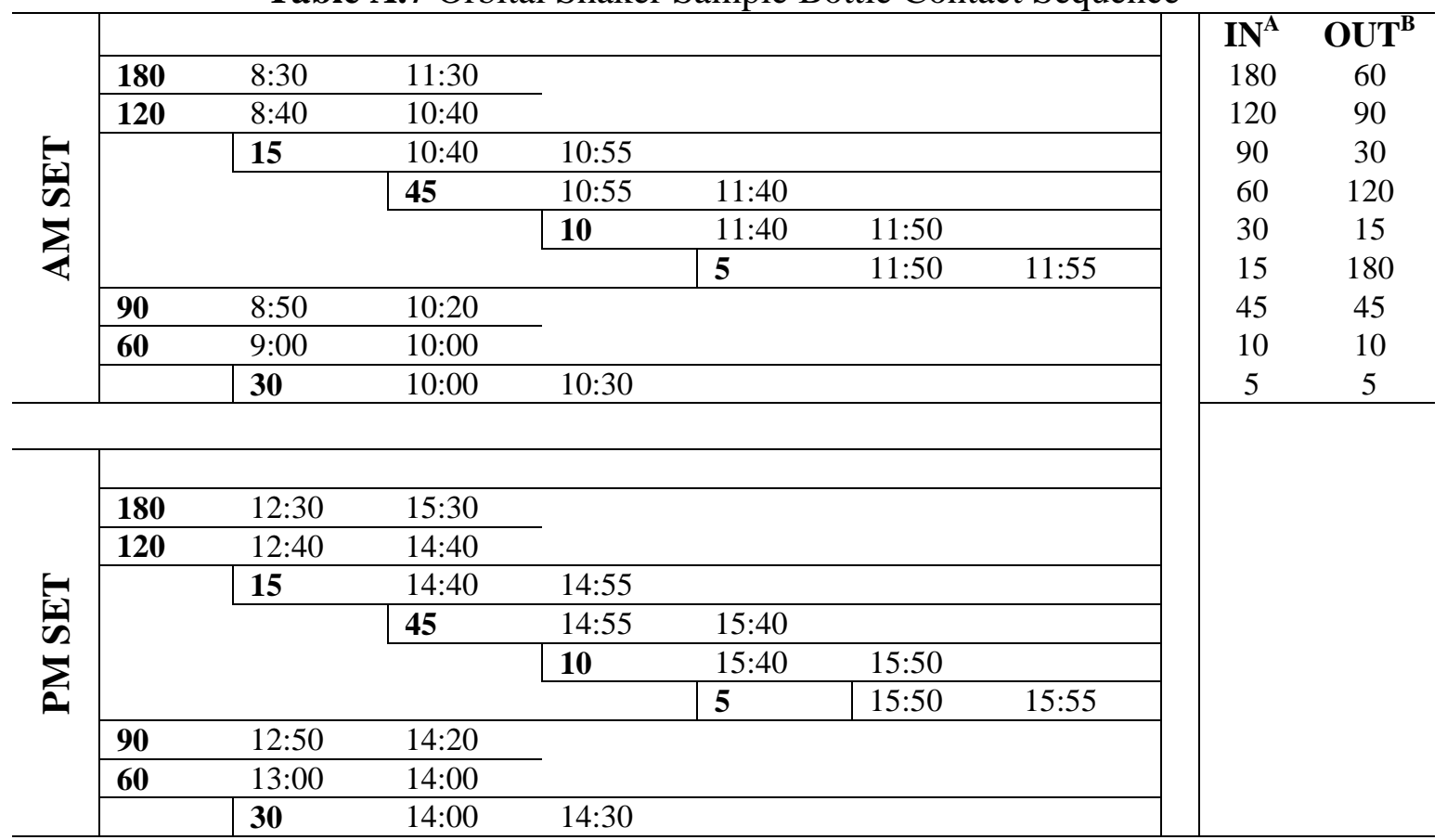

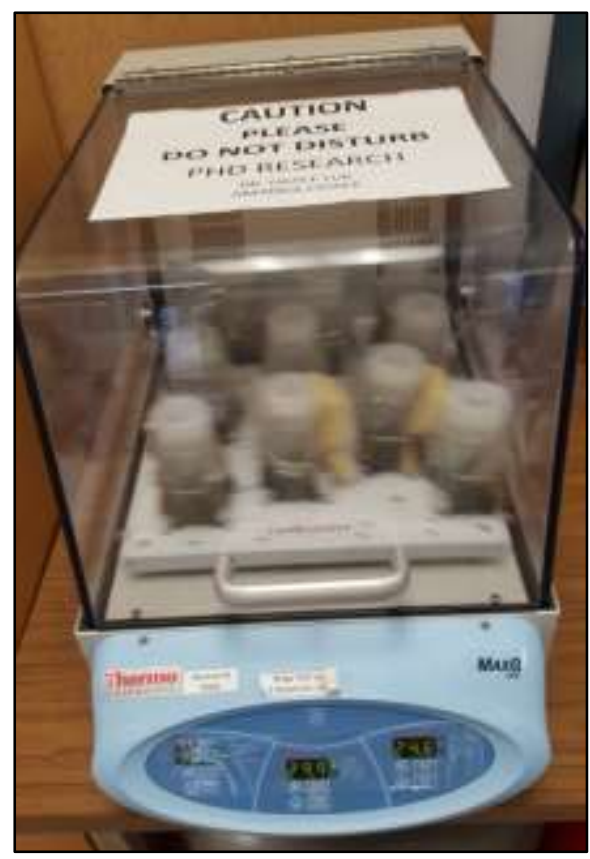

a. View of Sample Bottle Clamp Arrangement in Orbital Shaker

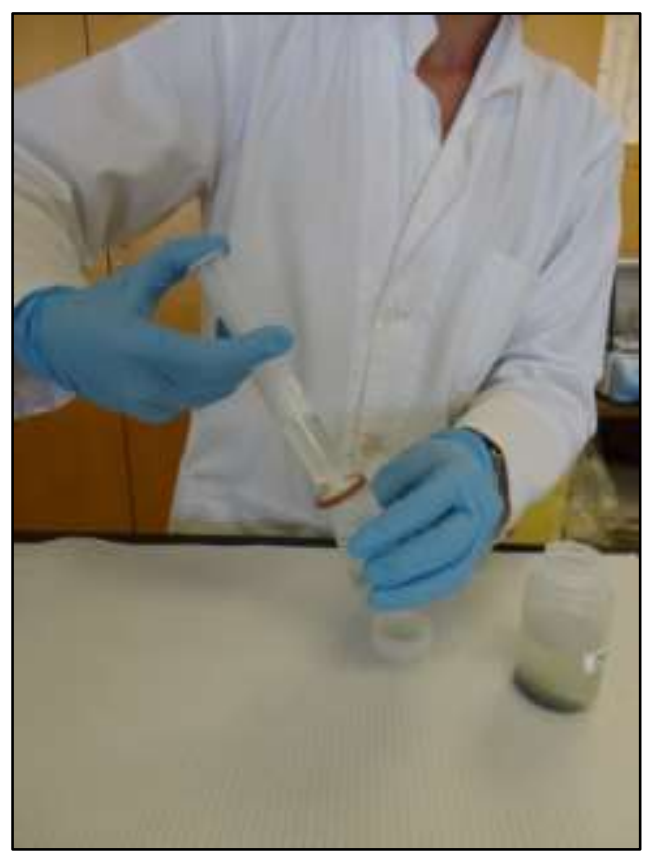

b. Separate Sorbate (HMI) and Sorbent (Zeolite) with Syringe-Filter

Figure A.6 Sorbate-Sorbent Contact and Separation 


\section{A.9. ICP-AES Sample Preparation}

- With reference to the lab sequence breakdown in Appendix A.3:

1. Collect deionized distilled water (DDW) $\left(18 \mathrm{~m} \Omega . \mathrm{cm} @ 25^{\circ} \mathrm{C}\right)$; approximately 4-L required for influent stock preparation contained in a $20 \mathrm{~L}$ carboy.

2. Label all ICP-AES 15-mL auto sampler tubes.

3. Prepare the ICP-AES standards by diluting the $100 \mathrm{mg} / \mathrm{L}$ Multi-Element Quality Control Standard 4 to total volumes of $10 \mathrm{~mL}$ at $1,10,50$, and $90 \mathrm{mg} / \mathrm{L}$, using the pipettes and DDW; set into the auto-sampler tubes (Table A.8)* (Figure A.7a).

4. Cap the tubes and invert slowly three (3) times.

5. Remove the $1 \mathrm{oz}$. sample bottles from the refrigerator.

6. Based on the component system concentration of each HMI of a given sequence, dilute to 20$\mathrm{mL}^{* *}$ total volumes into the $2 \mathrm{oz}$. sample bottles (Table A.9) (Figure A.7b).

7. Cap the bottles and invert slowly three (3) times.

8. Once all dilutions are complete, transfer the dilutions from the sample bottles to the prelabeled ICP-AES auto-sampler tubes.

9. Store ICP-AES auto-sampler tubes containing the diluted effluent samples in the refrigerator overnight.

10. With the residual effluent contained in the 1 oz. sample bottle, collect a pH level measurement.

11. Dispose of spent HMI effluent solution from bottles. 
Table A.8 ICP-AES Calibration Standards Dilutions

\begin{tabular}{ccc}
\hline $\begin{array}{c}\text { Standard Concentration } \\
(\mathbf{C} 2 ; \mathbf{m g} / \mathbf{L})\end{array}$ & $\begin{array}{c}\text { Standard Volume } \\
(\mathbf{V} \mathbf{1} ; \mathbf{m L})\end{array}$ & $\begin{array}{c}\text { DDW Volume } \\
(\mathbf{m L})\end{array}$ \\
\hline 100 & 10 & 0 \\
\hline 90 & 9 & 1 \\
\hline $50^{\mathrm{QC}}$ & 5 & 5 \\
\hline 10 & 1 & 9 \\
\hline 1 & 0.1 & 9.9 \\
\hline BLANK & 0 & 10 \\
\hline $\mathrm{C} 1=100 \mathrm{mg} / \mathrm{L}$ & & \\
$\mathrm{V} 2=10 \mathrm{~mL}$ & & \\
\hline
\end{tabular}

Table A.9 Number of 50\% Dilutions (DIL\#)*** for each Component System Sample

\begin{tabular}{c|c|c|c|c|c}
\hline \multirow{2}{*}{ SYSTEM } & \multicolumn{2}{c|}{ Samples } & \multirow{2}{*}{ SYSTEM } & \multicolumn{2}{c}{ Samples } \\
\cline { 2 - 3 } \cline { 5 - 6 } & $\mathrm{A}$ & $\mathrm{B}$ & & $\mathrm{A}$ & $\mathrm{B}$ \\
\hline$[\mathrm{P}]$ & 4 & 3 & {$[\mathrm{D}-\mathrm{PC}]$} & 3 & 2 \\
\hline$[\mathrm{C}]$ & 2 & 2 & {$[\mathrm{D}-\mathrm{PF}]$} & 3 & 2 \\
\hline$[\mathrm{F}]$ & 1 & 1 & {$[\mathrm{D}-\mathrm{PN}]$} & 3 & 2 \\
\hline$[\mathrm{N}]$ & 2 & 2 & {$[\mathrm{D}-\mathrm{PZ}]$} & 3 & 1 \\
\hline$[\mathrm{Z}]$ & 2 & 2 & {$[\mathrm{D}-\mathrm{CF}]$} & 1 & 1 \\
\hline$[\mathrm{T}]$ & 2 & 1 & {$[\mathrm{D}-\mathrm{CN}]$} & 1 & 1 \\
\hline$[\mathrm{M}]$ & 1 & 0 & {$[\mathrm{D}-\mathrm{FZ}]$} & 1 & 1 \\
\hline
\end{tabular}

${ }^{\mathrm{A}}$ Stock [S]; Contact Times: 5,10,15,30 min

${ }^{\mathrm{B}}$ Contact Times: 45,60,90,120,180 min

\begin{tabular}{|rcccc|}
\hline & DIL4 & DIL3 & DIL2 & DIL1 \\
V1 (Sample) $(\mathrm{mL})$ & 2.50 & 5.00 & 10.00 & 20.00 \\
DDW $(\mathrm{mL})$ & 37.50 & 35.00 & 30.00 & 20.00 \\
\hline
\end{tabular}

*Note - The calibration standards are disposed of (only one time use) and are prepared new to coincide with the sample preparations for every experimental week.

**Note - The number of $50 \%$ dilutions (DIL\#) is limited to $25-\mathrm{mL}$ syringe-filter capacity; where the residual $5-\mathrm{mL}$ is set for $\mathrm{pH}$ level measurements.

$* * *$ Note - The number of $50 \%$ dilutions (DIL\#) for the first phase of the research project investigating the particle size range and dosage applied an additional dilution step for 45-180 min samples; due to unknown zeolite sorption capacity at the onset of the research project. The dilutions (presented in Table A.9) have been refined for all preceding phases of the research project. 


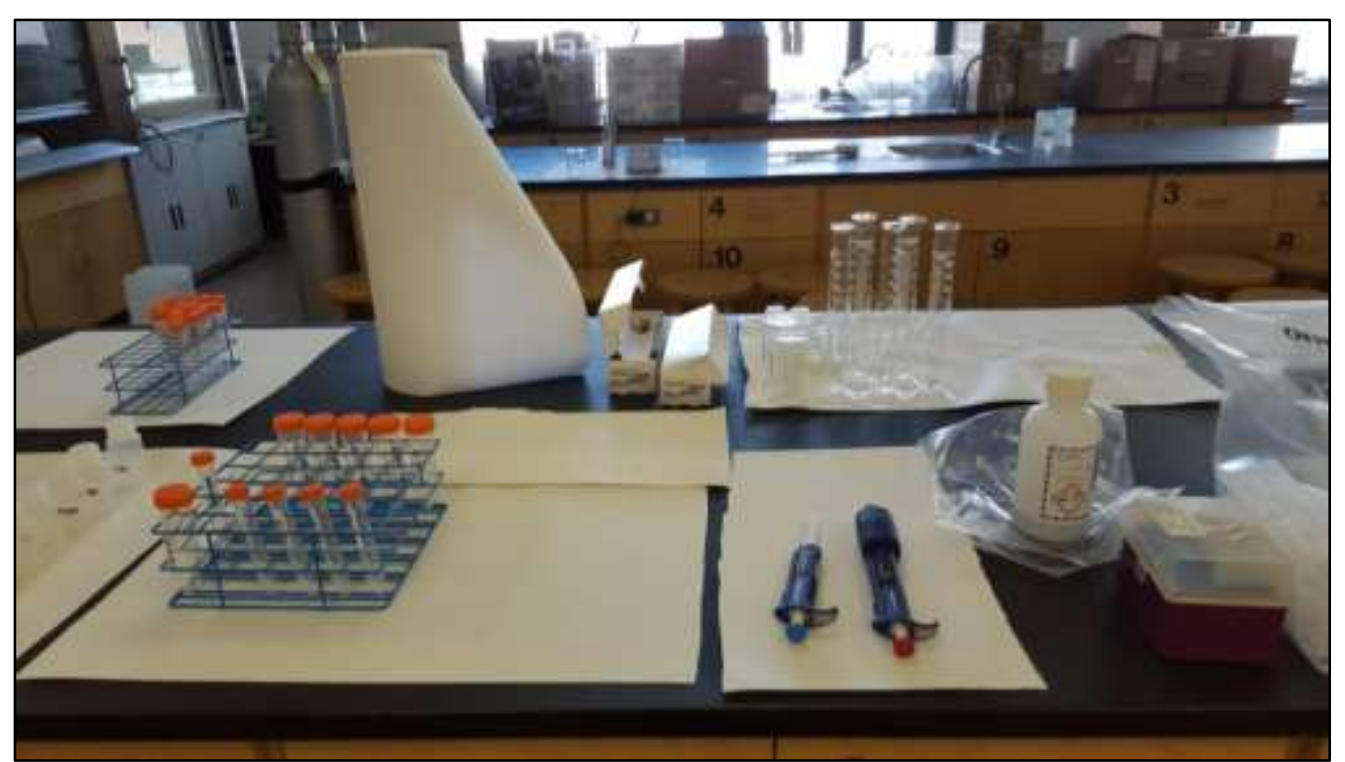

a. ICP-AES Sample Tubes and Preparation Station

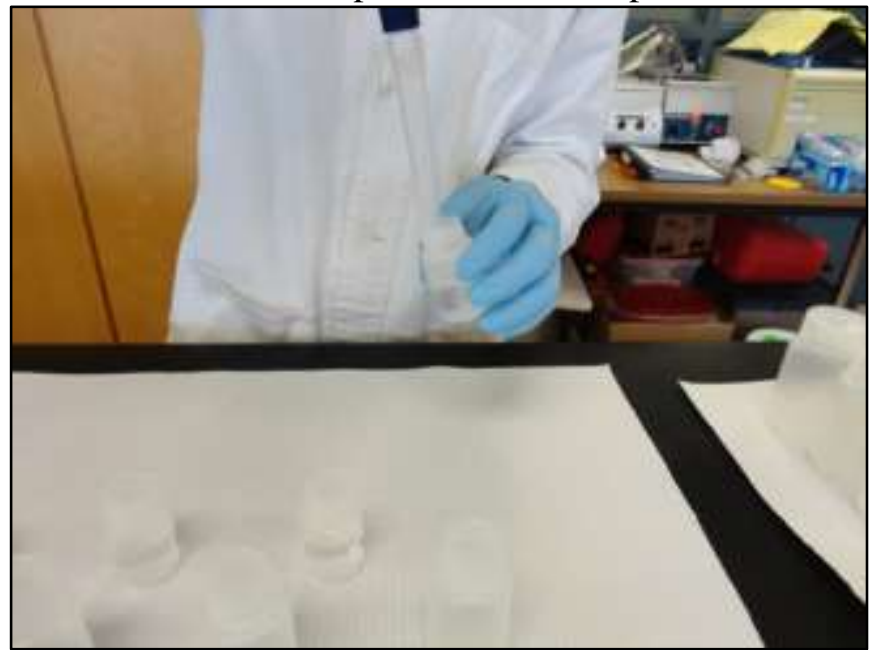

b. Dilute HMI Influent and Effluent Samples

Figure A.7 ICP-AES Elemental Analysis Preparation 


\section{A.10. Sorption System Development}

Figure A.8 displays the extensive optimization to reach the final sorption system design discussed in Chapter 7 and presented in Figure $\mathbf{7 . 2}$ of the Dissertation document. What began as a simple dual-column set-up with a peristaltic pump, evolved into the innovative development of custom sampling chambers with an automated controls system mounted on a rigid support system.
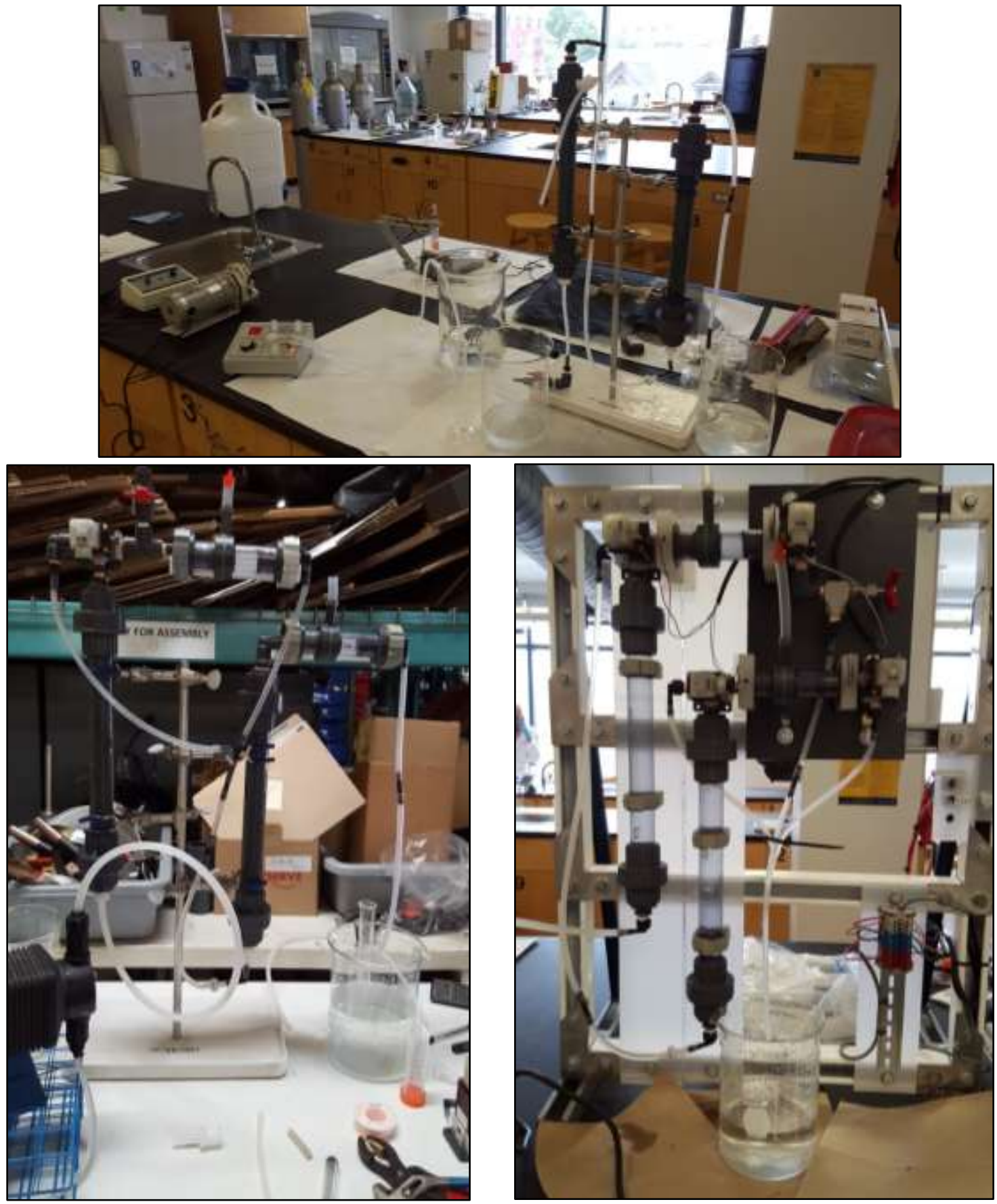

Figure A.8 Sorption System Prototype Development 


\section{APPENDIX B. ICP-AES Standard Operating Procedure}

\section{B.1. Instrument Sequence}

1. PLASMA ON 15 min before analysis

2. Method $>$ SET + CHECK $>$ Sample Info File (Same ID) $>$ SAVE *WINLAB32 Software Check Mark $\times 3>$ PROCEED

3. Workspace Display (Spectra - Calib - Analysis)

4. Automated Analysis Control

5. Flush PLASMA > Calibration CHECK > Flush PLASMA> Rebuild List> Analyze Samples

6. Reprocess Data $>$ EXPORT $>$ SAVE

7. PLASMA OFF

\section{B.2. Gas Valves}

- Air $(\mathrm{ON})$

- $\operatorname{Argon}(\mathrm{ON})$

- CHECK psi levels prior to analysis session

○ Direct Gas - 80/90 psi

○ Inlet Tank - 500 psi (minimum)

- Nitrogen

○ Adjust at each data run/test (1/4 turn counter-clockwise)

- Shut down > CLOSE

\section{B.3. Peristaltic Pump Set-Up}

- REFILL DDW

- Red/Red - Wash > Waste

○ Waste (CHECK > replace at end of week or flat/worn-out/foggy)

- Attached to nebulizer (back-most tube)

- Wash (DDW or $5 \% \mathrm{HNO}_{3}$ ) (analyte solution matrix); selected DDW to eliminate compounded error from unknown lab personal

- Black/Black (front-most tube)

- Sample > Top to nebulizer

- START - Replace with data set/lab visit

$\circ$ Pull taught and insert tubing $(2-3 \mathrm{~mm})$

- LOCK on the tubing clamps (check tubing goes into the groove in the clamp)

- MIST/SPRAY - Plasma ON (clockwise direction - movement)

○ Shut down > Release Tension

\section{B.4. Instrument Shut Down}

- Turn off Plasma Tab

- Wait until the gases stop flowing before you exit from the program.

- Exit from the program. Log out of Windows. Leave the computer on.

- Release clamps from the peristaltic sample pump, and release the tubing.

- Remove standards and samples from the $\mathrm{A} / \mathrm{S}$ tray. 


\section{B.5. New Method}

With Reference to Figure B.1a

- $\quad$ File $>$ New $>$ Method (or COPY another method)

$\circ$ Default (Aqueous Mode - 3-5\% TDS) $>$ Method Editor

\section{B.6. Instrument Start-up}

\section{Set A/S Tray}

- Place Blanks + Standards + Samples into the A/S tray

- Place into the well positions as specified in the Method and SIF

\section{Light Plasma}

With Reference to Figure B.1c

- Toolbar > Click "Plasma" button > Plasma Control box

- Click "Pump" button (turns on the sample pump)

- Verify that the solutions are flowing, and flowing in the right direction

- Click on the "On" button to light the plasma (15 mins before sequence)

First, it shuts off the sample pump, then it adjusts the gas flows, it lights the plasma, and then it turns the pump back on.

\section{B.7. Procedure Breakdown}

\section{Spectrometer}

A Define Elements

- Multi lines per multi elements

- BEC (Background Equivalent Concentration; ppm)

$\circ$ Preferential Order (5-10ppm)

- SELECT top 3 wavelengths (similar BEC levels/orders-of-magnitude)

*address interferences*

- Click "Wavelength Table" button to go to the list of elements and their wavelengths

- Control-click each line of the table that you want to import into your method, and then click on "Enter Selected Wavelengths in Method" button

\section{B Settings}

With Reference to Figure B.1b

- Purge Gas Flow - Normal

(High at <190 nm - based on elements' wavelengths - N/A)

- $\quad$ Read Time - 5-10 secs *Method optimization*

*Literature Reference - Typical Values per Calibration*

- Delay Time - 45 secs $>\sim 3$ mins/sample

- Replicated - Triplicate Runs (3)

$\circ$ one sample vial OR multi (time-dependent)

- Quantifiable Detection Limits - RSD

C Spectral Windows

- Find Emission Line (Default) 
2 Sampler - Power and Plasma View

A Plasma

- Mix Mode (Same or Vary to optimize method)

- FIRST ROW OVERRIDES ALL WAVELENGTHS*

- Radial $-1 \mathrm{ppm}>$ ('high' concentration) $>$ SELECT all radial

- Axial $-1 \mathrm{ppm}<(5 \times$ more sensitive $)$

- Equilibration Delay - 15 secs

- Plasma Conditions - Wet and Instant

- 1300 watts (SET 1500 watts to increase sensitivity via Axial)

B Peristaltic

- Rate $-1 \mathrm{~mL} / \mathrm{min}$

- Flush - 30 secs

C Auto Samples

- Frequency between Samples***

○ Rate $-1.5 \mathrm{~mL} / \mathrm{min}$

- Normal Time - 45 secs

- A/S Location WASH - Zero (0)

\section{Process}

A Peak Processing

- Peak Algorithm > Peak Area

- Points per Peak - 3-5

B Spectral Corrections

- 2 point

- BGC 1 \& 2 - Default - Change w/ Data

- Overlap Correction - NON

C Internal Standards - NO *click box*

- Internal Checks - Blank

\section{Calibration}

A Define Standards

- Calibration Blank

- Solvent Source (analyte Stock DDW) - MON412 LAB*

- Calibration Standard - A/S (auto-sampler) Location (Reagent Blank N/A)

- Click on the Calibration tab on the bottom.

- Give an ID and a location for each standard you will be using; ID what the element $i$, and its concentration

- A/S Number - NOTE Line Indicator

$B$ Calibration Units and Concentration

- Rank Set LOW > HIGH (NO ZEROS) - per order of magnitude in concentration*

○ Double-click column to set concentration* 


\section{Blank Usage}

- Calib Blank 1 (MON412 - DDW-S)

\section{Equation and Sample Units}

- Equations (adjust with data reprocessing)

○ Linear Calc. Int. (set to)

○ Through Zero

\section{E Initial Calibration and Multiline Calibration (Default)}

5 Checks

- $\quad$ Edit > Check Method

6 QC - Set Calibration Standard (Default)

\section{B.8. Save Method}

- $\quad$ Edit > CHECK Method

- File $>$ Save Method

- Main Page > File > Save As > Method

- Enter name $>\mathrm{OK}$

\section{B.9. Sample Info File}

With Reference to Figure B.1d

- Create a sample list

○ File > New > Sample Info File

○ Select "ppm design.sif" and click OK

- Fill in the table with the A/S location and sample ID for each sample > Batch ID - DATE

- Save your sample list

- File > Save As > Sample Info File

- Type in a name and click SAVE (as per saved Method)

- Close the Sample Information Editor 


\section{B.10. Automated Analysis Control}

\section{With Reference to Figure B.1e}

- Workspace File - CLICK:

- Spectra TAB

- Calib TAB

- Results TAB

- Options > Display>AutoLayout> per wavelength (1-3)

- Toolbar > Click "Auto" button (dialog box)

Set-Up TAB

○ Input Method

- Input Sample Info File

- CLICK > Use 'Sample Info' and SAVE DATA

- CLICK > 'Print Log During Analysis' OFF

Analyze TAB

- Click Calib BOX FIRST* ( 20 min)

- RSD < 3\% target

- Corr. Coeff $=0.999 \ldots$ (good correlation $)$

○ Click Rebuild List BOX

$>$ unknown (to analyze) show

○ *Plasma TAB > FLUSH (WASH) (15 seconds)

- Click Analyze Samples BOX

- Analysis Proceeds*

- Require $3 \mathrm{~min} / \mathrm{sample}$ (5\% error)

- FLUSH PLASMA ( 15 seconds IDLE) 


\section{B.11. Data Processing Analysis and Export}

\section{Examine}

- Examine $>$ Data $>$ Select Data Set $>$ Select All $>$ Next $>$ Select $>$ Finish ALL OK

- $\quad$ SELECT Primary Line

\section{Reprocess (Spectrum Alignment)}

- $\quad$ Toggle Peak/Endpoints $>$ Update Method Parameter > Set Peak > Update Method - CLOSE EXAMINE WINDOW

$$
\text { O File }>\text { Save Method }
$$

- Toolbar $>$ Reproc TAB > Browse Data Set $>$ Select Raw Data (ALL ROWS)

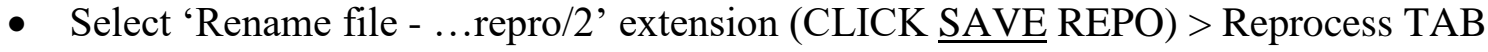

- Method > Calibration Equation> Through Zero > SAVE

- Examine Spectra

- Update Method

- Save Method $>$ REPO (SELECT ALL)

○ CALIB EQ $>$ TZ

- Save Method $>$ REPO TZ

- NOTE

- Reprocess curve $\mathrm{BC} 1+2$ and/or calibration curve relative to samples

- Toggle curve points and center peak to line

\section{Export}

- File $>$ Utilities $>$ Data Manager $>$ Select Data to import $>$ Select Export

- Create New Design > Next (OR existing design)

- Samples and Analytes to Export (make sure all data points and desired analytes selected > *ALL BOX CHECKED)

- All Unknowns (Select Primary Line) $>$ Next $>$ Extension '.prn' > TAB delimited

\section{Sample Parameters}

- Analyte/Sample ID (name)

- A/S Location

\section{Mean-Related Parameters}

- Analyte Name (Date and Time)

- Element Wavelength

- Intensity (corrected)

- Conc. - Calib Units

- \%-RSD (corr. Intensity)

- SD (corr. Intensity)

- Corr. Coeff. (corrected)
Replicate Parameters

- Number \# (Date and Time)

- Intensity (corrected)

- Conc. - Calib Units

- $\quad$ Save $>$ Export Design $>$ Save as data set (link ID/Name)

$\circ$ Open Excel $>$ File $>$ Open $>$ ALL FILES $>$ Save as '. $x 1 x$ ' 


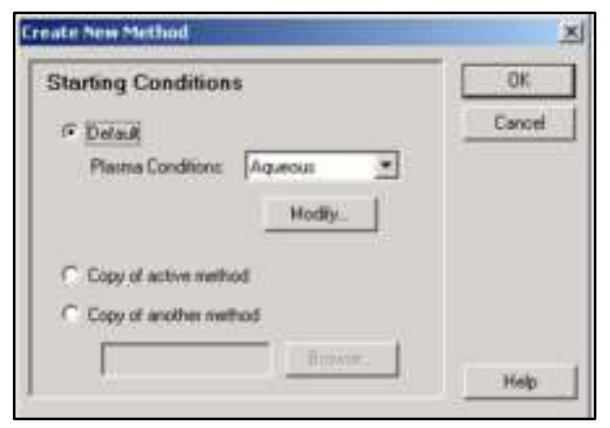

a. New Method

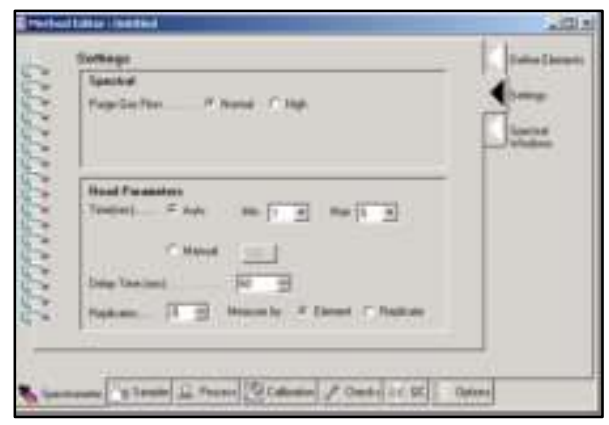

b. Spectrometer - B Settings
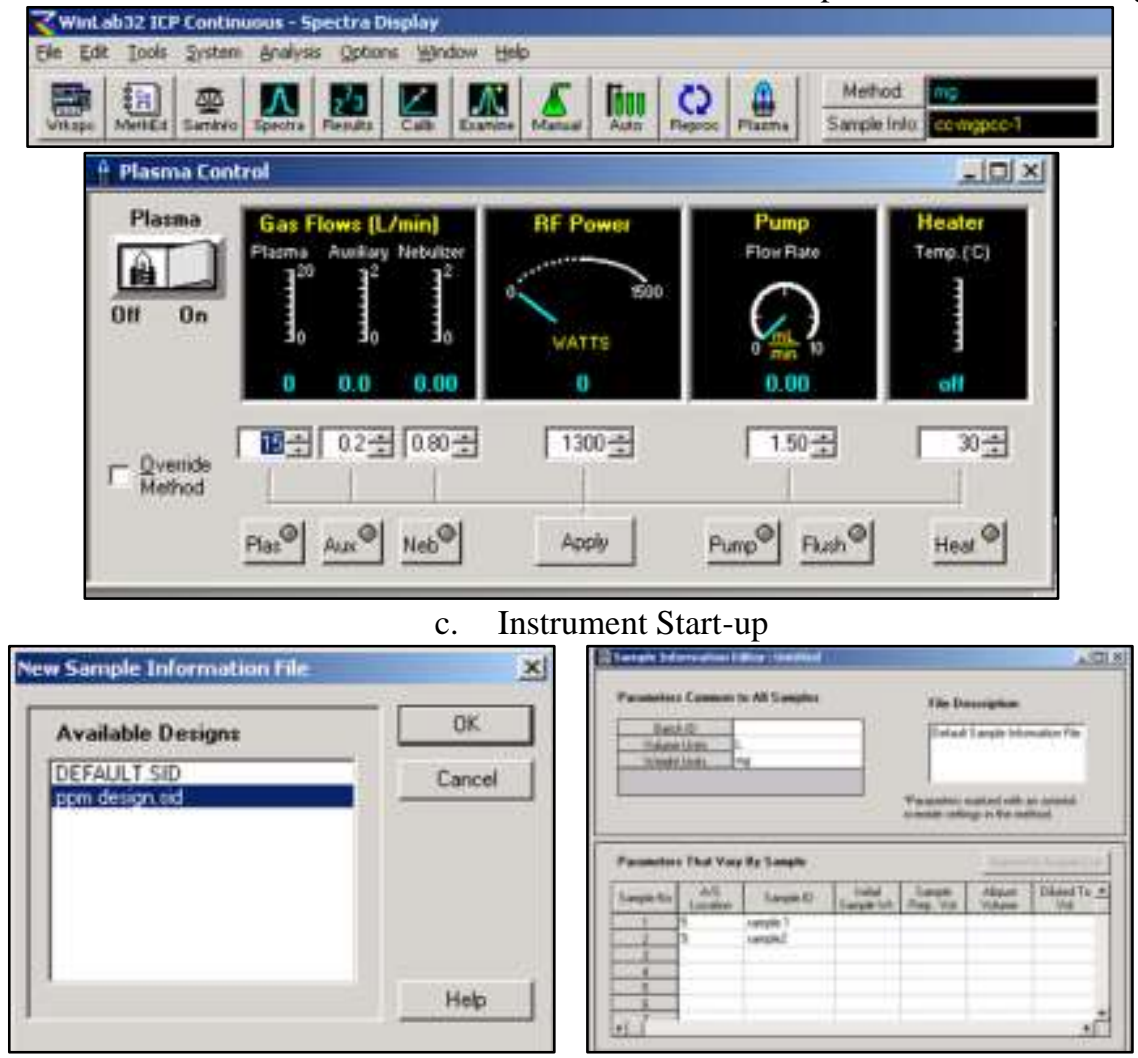

d. Sample Information File

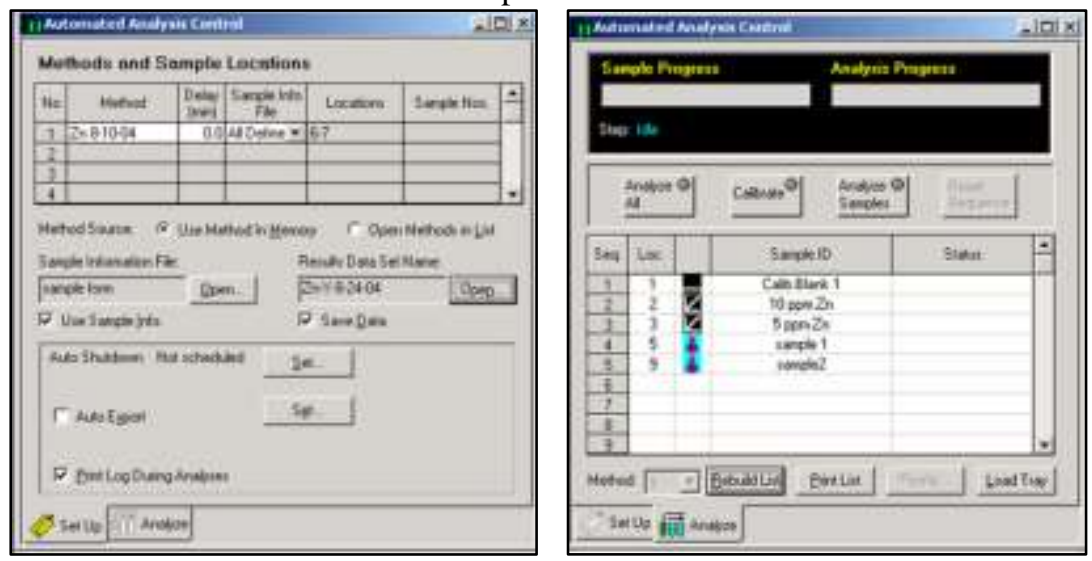

e. Automated Analysis Control

Figure B.1 ICP-AES WinLab 32 Software Interface (adapted from University of Michigan LSA, 2015) 


\title{
APPENDIX C. WEAO2016 Technical Symposium \\ A Study on the Effects of Multiple Metallic Ions on the Zeolite Adsorption of Lead from Mine Wastewater \\ Technical Session: Industrial Treatment A; Technical Session No.: 14 \\ Paper No: 2016-03; 12 April 2016.
}

\begin{abstract}
This study investigates the ionic effects of $\mathrm{Cu}^{2+}, \mathrm{Fe}^{3+}, \mathrm{Ni}^{2+}$ and $\mathrm{Zn}^{2+}$ in multi-component systems, onto the adsorption of $\mathrm{Pb}^{2+}$ by natural zeolite in the form of clinoptilolite. The objective is to determine the overall adsorption of the target metal lead and the selectivity of clinoptilolite to the various heavy metallic ions (HMI) combined in the aqueous phase. Elemental analysis conclude that after just 3 hours of contact time, the target metal ion lead is removed by 77.5, 88.6, 78.3, 86.4, and 91.5\% in the single-lead, dual-copper, dual-iron, triple-(lead-copper-iron), and multi-component systems, respectively. The removal efficiency order or 'selectivity series' is determined as $\mathrm{Pb}^{2+}>>\mathrm{Fe}^{3+}>\mathrm{Cu}^{2+}>\mathrm{Zn}^{2+}>\mathrm{Ni}^{2+}$.
\end{abstract}




\title{
APPENDIX D. WEAO2017 Technical Symposium \\ Effects of Heat Pre-Treatment on Metallic Ion Adsorption and Ion-Exchange by Natural Zeolite
}

Technical Session: Industrial Treatment A; Technical Session No.: 4

Paper No: 2017-010; 03 April 2017.

\begin{abstract}
This study investigates the effects of heat pre-treatment on natural zeolite in its sorptive removal efficiency of the heavy metallic ions (HMIs) $\mathrm{Cu}^{2+}, \mathrm{Fe}^{3+}, \mathrm{Ni}^{2+}, \mathrm{Pb}^{2+}$, and $\mathrm{Zn}^{2+}$. The ionic effects of these HMIs were examined in triple- [T] and multi-[M] component systems of a total $0.01 \mathrm{~N}$ (10 meq/L) influent concentration. After 3 hours of contact time, the removal efficiency order is sustained throughout heat pre-treatment analysis to be $\mathrm{Pb}^{2+}>>\mathrm{Fe}^{3+}>\mathrm{Cu}^{2+}>\mathrm{Zn}^{2+}>\mathrm{Ni}^{2+}$. When the $\mathrm{Ni}^{2+}$ and $\mathrm{Zn}^{2+}$ ions are introduced to the triple-[T] system, the $\mathrm{Pb}^{2+}$ ion and total HMI uptake is interfered in the multi-[M] system by a reduction of $37.5 \%$ and $25.3 \%$ [non-heated], $36.2 \%$ and $24.4 \%$ [200 ${ }^{\circ} \mathrm{C}$ ], $30.4 \%$ and $19.2 \%$ [400 ${ }^{\circ} \mathrm{C}$ ], and $25.9 \%$ and $25.6 \%\left[600^{\circ} \mathrm{C}\right.$, respectively. The total uptake of HMIs by zeolite is reduced by $6.1 \%$ [T] and $5.0 \%[\mathrm{M}]$ at $200^{\circ} \mathrm{C}, 33.2 \%$ [T] and $27.8 \%[\mathrm{M}]$ at $400^{\circ} \mathrm{C}$, and $46.8 \%[\mathrm{~T}]$ and $47.0 \%[\mathrm{M}]$ at $600^{\circ} \mathrm{C}$ exposure; compared to the nonheat-pre-treated form. This research has demonstrated that no major improvement of HMI sorption is achieved with progressive heat pre-treatment of zeolite.
\end{abstract}




\title{
APPENDIX E. WEAO2018 Technical Symposium \\ An Innovative Sorption Treatment System to Remove \\ Heavy Metallic Ions from Mine Wastewater \\ Technical Session: Industrial Treatment B; Technical Session No.: 26 \\ Paper No: 2018-072; 17 April 2018.
}

\begin{abstract}
This study demonstrates the performance of a sorption system prototype, consisting of fixed-bed, dual-columns packed with natural zeolite, custom sampling chambers and automated controls. The system is fed continuously with a synthetic simple solute aqueous solution of a total 10 meq/L multi-component heavy metallic ion (HMI) influent concentration; by a controlled adjustable distribution timer. The sorption process of five HMIs commonly found in industrial wastewater effluent is evaluated. The system removal efficiency is demonstrated by a selectivity order of lead $\left(\mathrm{Pb}^{2+}\right)>$ iron $\left(\mathrm{Fe}^{3+}\right)>\operatorname{copper}\left(\mathrm{Cu}^{2+}\right)>\operatorname{zinc}\left(\mathrm{Zn}^{2+}\right)>>$ nickel $\left(\mathrm{Ni}^{2+}\right)$. The lead ion is removed completely throughout the 3-hour contact period in both columns. An overall higher bed capacity at breakthrough and usage rate in the second column is observed. This system prototype experimentally contributes to the advancement of industrial wastewater treatment technology.
\end{abstract}




\title{
APPENDIX F. Water Environment Research Journal Publication
}

Prior to the in-depth research into the removal of heavy metallic ions of this research project, the capacity of natural zeolite to address high nutrient levels was investigated by the Ph.D. Candidate in the M.A.Sc. research. The objective of the study was to target the removal of total phosphorus (TP), and to demonstrate the efficiency of an optimized clay-zeolite medium. The outcome of this study compared well to other common removal technologies, as a more simplistic and affordable system alternative. With further optimization, this system would minimize direct surface discharge of phosphorus and overall, contributes to the advancement of eutrophication control. The journal publication reference details (Figure F.1) are as follows.

\section{An Innovative Design of a Clay-Zeolite Medium for the Adsorption of Total Phosphorus from Wastewater} Amanda Lidia Ciosek ${ }^{1 *}$, Grace K. Luk ${ }^{2}$, Michèle Warner ${ }^{3}$, R. Anthony Warner ${ }^{4}$ ${ }^{1 * 2}$ Department of Civil Engineering, Faculty of Engineering and Architectural Science, Ryerson University, Toronto, Ontario, Canada.

${ }^{3,4}$ Virtual Engineers Inc., Markham, Ontario, Canada.

\section{Water Environment Research}

Volume 88, Number 2; 01 February 2016; 131-142(12). doi:10.2175/106143015X14338845155787.

\begin{abstract}
Phosphorus significantly influences the eutrophication process, modifying the quality of waterways and habitat, especially in stagnant water bodies exposed to septic tank effluent at high nutrient levels. This research explores the development of a cost-effective, efficient, and affordable on-site wastewater treatment system targeted as total phosphorus (TP) removal technology. The research objective is to demonstrate the TP removal efficiency of an optimized clay-zeolite medium by chemical adsorption. The study observes the effects of pellet medium design and modifications, influent concentrations, and contact time. Following various stages of optimization, the preliminary testing achieves a $45 \pm 1.8 \%$ removal after 45 minutes of contact time. The optimized pellets are contained within a five-layer bench-scale model, achieving equilibrium TP removal of $72 \pm 2.9 \%$ after 3 hours. Theoretical extrapolation to 12 contact hours indicates an achievement of $88 \%$ removal is possible. The results show a positive correlation with the linearized Langmuir and Freundlich adsorption isotherms.
\end{abstract}

Keywords: wastewater treatment, total phosphorus, clay, zeolite, Langmuir, Freundlich, adsorption, isotherm. 


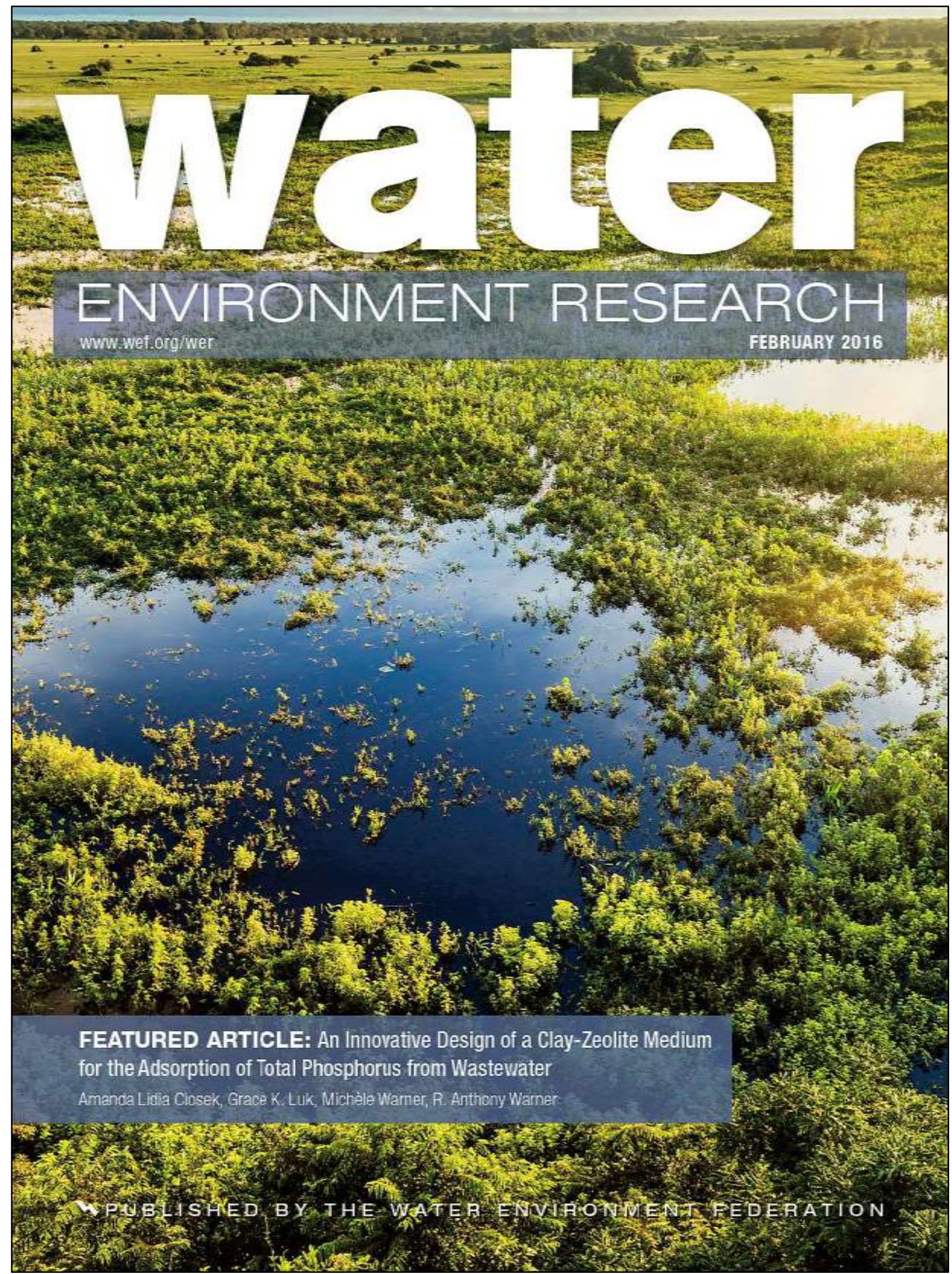

Figure F.1 Water Environment Research Journal Publication - Cover Featured Research Article 


\section{REFERENCES}

1. Acheampong, M.A.; Meulepas, R.J.W.; Leans, P.N.L. Removal of heavy metals and cyanide form gold mine wastewater. J. Chem. Technol. Biotechnol. 2010, 85, 590-613, doi:10.1002/jctb.2358.

2. Acheampong, M.A. Sustainable Gold Mining Wastewater Treatment by Sorption Using Low-Cost Materials. Ph.D. Thesis, UNESCO-IHE Institute of Water Education, Delft, The Netherlands, 2013; ISBN 978-1-138-00165-7.

3. Adams, M. Cyanide recovery by tailings washing and pond stripping. Miner. Eng. 2008, 21, 501-508, doi:10.1016/j.mineng.2008.02.015.

4. Akcil, A.; Koldas, S. Acid Mine Drainage (AMD): Causes, treatment and case studies. $J$. Clean. Prod. 2006, 14, 1139-1145, doi:10.1016/j.jclepro.2004.09.006.

5. Akkoca, D.B.; Yilgin, M.; Ural, M.; Akcin, H.; Mergen, A. Hydrothermal and Thermal Treatment of Natural Clinoptilolite Zeolite from Bigadiç, Turkey: An Experimental Study. Geochemistry International. 2013, 51(6), 495-504, 2013. doi:10.1134/S0016702913040022.

6. Akpor, O.B.; Ohiobor, G.O.; Olaolu, T.D. Heavy metal pollutants in wastewater effluents: Sources, effects and remediation. Adv. Biosci. Bioeng. 2014, 2(4), 37-43, doi:10.11648/j.abb.20140204.11.

7. Alvarez-Ayuso, E.; Garcia-Sanchez, A.; Querol, X. Purification of metal electroplating waste waters using zeolites. Water Res. 2003, 37, 4855-4862, doi:10.1016/j.watres.2003.08.009.

8. Anari-Anaraki, M.; Nezamzadeh-Ejhieh, A. Modification of an Iranian clinoptilolite nanoparticles by hexadecyltrimethyl ammonium cationic surfactant and dithizone for removal of $\mathrm{Pb}$ (II) from aqueous solution. J. Colloid Interf. Sci. 2015, 440, 272-281, doi:10.1016/j.jcis.2014.11.017.

9. Andoh, R. (2014) Filter Technology is Looking Up - Storm Water Solutions. Available online: www.estormwater.com/filter-technology-looking (accessed on 04 March 2014).

10. ASTM D2434-68. Standard Test Method for Permeability of Granular Soils (Constant Head). ASTM International, West Conshohocken, PA. 2000. Available online: www.astm.org (accessed on 01 March 2016).

11. Asubiojo, O.I.; Ajelabi, O.B. Removal of heavy metals from industrial wastewaters using natural adsorbents. Toxicol. Environ. Chem. 2009, 91(5), 883-890, doi:10.1080/02772240802614721.

12. Babel, S., Kurniawan, T.A. Low-cost adsorbents for heavy metals uptake from contaminated water: a review. J. Hazard. Mater. 2003, 97, 219-243, doi:S0304-3894(02)00263-7.

13. Baraket, M. A. New trends in removing heavy metals from industrial wastewater. Arab. J. Chem. 2011, 4(4), 361-377doi:10.1016/j.arabjc.2010.07.019.

14. Bear River Zeolite Co. Inc. Zeolite-Specifications and MSDS. Available online: www.bearriverzeolite.com (accessed on 01 September 2012 and 01 April 2017). 
15. Behnamfard, A.; Salarirad, M. M. Equilibrium and kinetic studies on free cyanide adsorption from aqueous solution by activated carbon. J. Hazard. Mater. 2009, 170, 127133, doi:10.1016/j.jhazmat.2009.04.124.

16. Benjamin, M.M. Water Chemistry, $2^{\text {nd }}$ ed.; Waveland Press, Inc. 2010, Inc. ISSN 978-14786-2308-3.

17. Bekkum, H.V.; Flanigen, E.M.; Jansen, J.C. Ion-Exchange in Zeolites. In Introduction to Zeolite Science and Practice-Studies in Surface Science and Catalysis, 1st ed.; Elsevier Science: Zeist, The Netherlands, 1991; Volume 58, pp. 359-390.

18. Bektas, N.; Kara, S. Removal of lead from aqueous solutions by natural clinoptilolite: Equilibrium and kinetic studies. Sep. Purif. Technol. 2004, 39, 189-200, doi:10.1016/j.seppur.2003.12.001.

19. Borandegi, M.; Nezamzadeh-Ejhieh, A. Enhanced removal efficiency of clinoptilolite nanoparticles toward $\mathrm{Co}(\mathrm{II})$ from aqueous solution by modification with glutamic acid. Colloids Surfaces A Physicochem. Eng. Asp. 2015, 479, 35-45, doi:10.1016/j.colsurfa.2015.03.040.

20. Bosso, S.T.; Enzweiler, J. Evaluation of heavy metal removal from aqueous solution onto scolecite. Wat. Res. 2002, 36, 4795-4800, doi:10.1016/S0043-135400208-7.

21. Breck, D.W. Chapters 2-4, 6-8; Zeolite Molecular Sieves: Structure, Chemistry, and Use. John Wiley \& Sons, New York, 1974.

22. Cabrera, C.; Gabaldon, C.; Marzal, P. Technical Note: Sorption characteristics of heavy metal ions by natural zeolite. J. Chem. Technol. Biotechnol. 2005, 80, 477-481, doi:10.1002/jctb.1189.

23. Çakıcğlu-Ozkan, F.; Ülkü, S. Diffusion mechanism of water vapour in a zeolitic tuff rich in clinoptilolite. J. Therm. Anal. Calorim. 2008, 94(3), 699-702, doi:10.1007/s10973-0089357-8.

24. Canada-United States Collaboration for Great Lakes Water Quality. About the Great Lakes Water Quality Agreement. Available online: binational.net/glwqa-aqegl/ (accessed on 20 April 2018).

25. Canadian Minister of Justice-Metal Mining Effluent Regulations. Consolidation SOR/2002-222. Justice Laws-Government of Canada. Available online: lawslois.justice.gc.ca (accessed on 01 September 2014).

26. Chemistry LibreTexts. The Rate Law. 2015. Available online: chem.libretexts.org/Core/Physical_and_Theoretical_Chemistry/Kinetics/Rate_Laws/The_Ra te_Law (accessed on 01 May 2018).

27. Chmielewská, E.; Sabová, L.; Jesenák, K. Study of adsorption phenomena ongoing onto clinoptilolite with the immobilized interfaces. J. Therm. Anal. Calorim. 2008, 92(2), 567571, doi:10.1007/s10973-006-8315-6.

28. Cincotti, A.; Mameli, A.; Locci, A.M.; Orru, R.; Cao, G. Heavy metals uptake by Sardinian natural zeolites: Experiment and modeling. Ind. Eng. Chem. Res. 2006, 45, 1074-1084, doi:10.1021/ie050375z. 
29. Ciosek, A. L.; Luk, G. K. A Study on the Effects of Multiple Metallic Ions on the Zeolite Adsorption of Lead from Mine Wastewater. Technical Session: Industrial Treatment A (14), Water Environment Association of Ontario Technical Symposium and OPCEA Exhibition Niagara Falls, Ontario, Canada. Paper No.: 2016-03. 2016.

30. Ciosek, A.L.; Luk, G.K. Lead Removal from Mine Tailings with Multiple Metallic Ions. Int. J. Water Wastewater Treat. 2017a, 3, 1-9, doi:10.16966/2381-5299.134.

31. Ciosek, A. L.; Luk, G. K. Effects of Heat Pre-Treatment on Metallic Ion Sorption by Natural Zeolite. Technical Session: Industrial Treatment A (4), Water Environment Association of Ontario Technical Symposium and OPCEA Exhibition - Ottawa, Ontario, Canada. Paper No.: 2017-010. 2017b.

32. Ciosek, A. L.; Luk, G. K. Kinetic Modelling of the Removal of Multiple Heavy Metallic Ions in Mine Waste by Natural Zeolite Sorption. Special Issue: Treatment of Wastewater and Drinking Water through Advanced Technologies, In Water. 2017c, 9(7), 482, doi:10.3390/w9070482.

33. Ciosek, A. L.; Luk, G. K. An Innovative Dual-Column System for Heavy Metallic Ion Sorption by Natural Zeolite. Special Issue: Wastewater Treatment and Reuse Technologies, In Applied Sciences. 2017d, 7(8), 795, doi: 10.3390/app7080795.

34. Ciosek, A.L.; Luk, G.K. Effects of Operation Parameters on Heavy Metallic Ion Removal from Mine Waste by Natural Zeolite. Int. J. Environ. Pollution and Remediation. 2018, 6(1), 10-24, doi:10.11159/ijepr.2018.002.

35. Ciosek, A. L. An Innovative Sorption Treatment System to Remove Heavy Metallic Ions from Mine Wastewater. Technical Session: Industrial Treatment B (26), Water Environment Association of Ontario Technical Symposium and OPCEA Exhibition - London, Ontario, Canada. Paper No.: 2018-072. 2018.

36. Cui, H.; Li, L.Y.; Grace, J.R. Exploration of remediation of acid rock drainage with clinoptilolite as sorbent in a slurry bubble column for both heavy metal capture and regeneration. Water Res. 2006, 3359-3366, doi:10.1016/j.watres.2006.07.028.

37. Curenature. Heavy Metal Toxicity \& Effective Chelators. 2018. Available online: www.curenature.com/2013/03/heavy-metal-toxicity-effective-chelators.html (accessed on: 01 September 2014).

38. Curkovic, L.; Cerjan-Stefanovic, S.; Filipan, T. Metal ion exchange by natural and modified zeolites. Wat. Res. 1997, 31, 1379-1382, doi:10.1016/S0043-135400411-3.

39. Delgado, J.M.P.Q. A critical review of dispersion in packed beds. Heat Mass Transfer. 2005, 42(4), 279-310, doi:10.1007/s00231-005-0019-0.

40. Dinardo, O.; Kondos, P.D.; MacKinnon, D.J.; McCready, R.G.L.; Riveros, P.A.; Skaff, M. Study on Metals Recovery/Recycling from Acid Mine Drainage Phase IA: Literature Survey. Report 3.21.1a. Mine Environment Neutral Drainage (MEND) Program. CANMET, Energy, Mines and Resources Canada and WTC, Environment Canada. 1991. Available online: mend-nedem.org/wp-content/uploads/2013/01/3.21.1a.pdf (accessed on 30 October 2014). 
41. Dridi-Dhaouadi, S.; Douissa-Lazreg, N. B.; M'Henni, M. F. Removal of lead and Yellow 44 acid dye in single and binary component systems by raw Posidonia oceanica and the cellulose extracted from the raw biomass. Environ. Technol. 2011, 32(3), 325-340, doi:10.1080/09593330.2010.499545.

42. Droste, R.L. Theory and Practice of Water and Wastewater Treatment, 1st ed., Wiley \& Sons, 1997.

43. Engels, J. Tailings Info. 2013. Available online: www.tailings.info/index.htm (accessed on 07 March 2014).

44. Erdem, E.; Karapinar, N.; Donat, R. The removal of heavy metal cations by natural zeolites. J. Colloid Interface Sci. 2004, 280, 309-314, doi:10.1016/j.jcis.2004.08.028.

45. Erdol Aydin, N.; Nasun Saygili, G. Column experiments to remove copper from wastewaters using natural zeolite. Int. J. Environment and Waste Management. 2009, 3, 319-326, doi:10.1504/IJEWM.2009.026348.

46. Ersoy, B.; Celik, M.S. Electrokinetic properties of clinoptilolite with mono- and multivalent electrolytes. Micropor. Mesopor. Mat. 2002, 55, 305-312, doi:10.1016/S1387-181100433$\mathrm{X}$.

47. Farouq, R.; Yousef, N.S. Equilibrium and Kinetics Studies of adsorption of Copper (II) Ions on Natural Biosorbent. Int. J. Chem. Eng. Applic. 2015, 6(5), 319-324, doi:10.7763/ijcea.2015.v6.503.

48. Fierro, V.; Torne-Fernandez, V.; Montane, D.; Celzard, A. Adsorption of phenol onto activated carbons having different textural and surface properties. Micropor. Mesopor. Mat. 2008, 111, 276-284, doi:10.1016/j.micromeso.2007.08.002.

49. Fu, F.; Wang, Q. Removal of heavy metal ions from wastewaters: A review. J. Environ. Manag. 2011, 92, 407-418, doi:10.1016/j.jenvman.2010.11.011.

50. Garbarino, J. R.; Hayes, H. C.; Roth, D. A.; Antweiler, R. C.; Brinton, T. I.; Taylor, H. E. Heavy Metals in the Mississippi River. Contaminants in the Mississippi River - U.S. Geological Survey Circular 1133. 1996. Available online: http://pubs.usgs.gov/circ/circ1133/heavy-metals.html (accessed on 08 August 2014).

51. Gregory, R. Filtration Processes. IWA Water Wiki. Information Resource and Hub for the Global Water Community. 2010. Available online: http://www.iwawaterwiki.org/xwiki/bin/export/Articles/FiltrationProcesses?format=pdf\&pd fcover $=1 \&$ pdfcover $=0 \&$ pdftoc $=1 \&$ pdftoc $=0 \&$ comments $=0 \&$ attachments $=0$ (accessed on 04 March 2014).

52. Gupta, N.; Balomajumder, C.; Agarwal, V. K. Adsorption of cyanide ion on pressmud surface-A modeling approach. Chem. Eng. J. 2012, 191, 549-556, doi:10.1016/j.cej.2012.03.028.

53. Gunay, A.; Arslankaya, E.; Tosun, I. Lead removal from aqueous solution by natural and pretreated clinoptilolite: Adsorption equilibrium and kinetics. J. Hazard. Mater. 2007, 146, 362-371, doi:10.1016/j.jhazmat.2006.12.034.

54. Gunn, D.J. Axial and Radial Dispersion in Fixed Beds. Chem. Eng. Sci. 1987, 42(2), 363373, doi:10.1016/0009-2509(87)85066-2. 
55. Han, R.; Zou, W.; Li, H.; Li, Y.; Shi, J. Copper(II) and lead(II) removal from aqueous solution in fixed-bed columns by manganese oxide coated zeolite. J. Hazard. Mater. 2006, 137, 934-942, doi:10.1016/j.jhazmat.2006.03.016.

56. Haynes, W. M. CRC Handbook of Chemistry and Physics. 95th Edition 2014-2015. Available online: www.hbcpnetbase.com.ezproxy.lib.ryerson.ca/ (accessed on 13 November 2014).

57. Helfferich, F. Chapter 5, 6, 9. In Ion Exchange; Series in Advanced Chemistry; McGrawHill Book Company: New York, USA, 1962; pp. 95-322; 421-506.

58. Ho, Y.S.; McKay, G. A Comparison of Chemisorption Kinetic Models Applied to Pollutant Removal on Various Sorbents. Process. Saf. Environ., 1998a, 76(4), 332-340, doi:10.1205/095758298529696.

59. Ho, Y.S.; McKay, G. Kinetic Models for the Sorption of Dye from Aqueous Solution by Wood. Process. Saf. Environ. 1998b, 76(2), 183-191, doi:10.1205/095758298529326.

60. Ho, Y.; Ofomaja, A.E. Pseudo-second-order model for lead ion sorption from aqueous solutions onto palm kernel fiber. J. Hazard. Mater. 2006, 129, 137-142, doi:10.1016/j.jhazmat.2005.08.020.

61. Huang, M.; Kalliagunie, S.; Auroux, A. Lewis basic and Lewis acidic sites in zeolites. Stud. Surf. Sci. Catal. 1995, 97, 311-318, doi:10.1016/S0167-2991(06)81903-8.

62. Imperial Metals Corporation (IMC). Mount Polley Mine - Overview. Available online: www.imperialmetals.com/s/MountPolleyMine.asp (accessed on 01 September 2014).

63. Inglezakis, V.J.; Papadeas, C.D.; Loizidou, M.D.; Grigoropoulou, H.P. Effects of Pretreatment on Physical and Ion Exchange Properties of Natural Clinoptilolite. Environ. Technol. 2001a, 22, 75-82, doi:10.1080/09593332208618308.

64. Inglezakis, V.J.; Hadjiandreou, K.J.; Loizidou, M.D.; Grigoropoulou, H.P. Pretreatment of Natural Clinoptilolite in a Laboratory-Scale Ion Exchange Packed Bed. Water Res. 2001b, 35, 2161-2166, doi:10.1016/S0043-135400500-5.

65. Inglezakis, V.J.; Loizidou, M.D.; Grigoropoulou, H.P. Equilibrium and kinetic ion exchange studies of $\mathrm{Pb}^{2+}, \mathrm{Cr}^{3+}, \mathrm{Fe}^{3+}$ and $\mathrm{Cu}^{2+}$ on natural clinoptilolite. Water Res. 2002, 36, 27842792, doi:10.1016/S0043-135400504-8.

66. Inglezakis, V.J.; Loizidou, M.D.; Grigoropoulou, H.P. Ion exchange of $\mathrm{Pb}^{2+}, \mathrm{Cu}^{2+}, \mathrm{Fe}^{3+}$, and $\mathrm{Cr}^{3+}$ on natural clinoptilolite: Selectivity determination and influence of acidity on metal uptake. J. Colloid Interface Sci. 2003, 261, 49-54, doi:10.1016/S0021-979700244-8.

67. Inglezakis, V. J.; Grigoropoulou, H. Effects of operating conditions on the removal of heavy metals by zeolite in fixed bed reactors. J. Hazard. Mater. 2004, 112, 37-43, doi:10.1016/j.jhazmat.2004.02.052.

68. Inglezakis, V. J. The concept of "capacity" in zeolite ion-exchange systems. J. Colloid Interface Sci. 2005, 281, 68-79, doi:10.1016/j.jcis.2004.08.082.

69. Inglezakis, V.J.; Zorpas, A.A., Loizidou, M.D.; Grigoropoulou, H.P. The effect of competitive cations and anions on ion exchange of heavy metals. Sep. Purif. Technol. 2005, 46, 202-207, doi:10.1016/j.seppur.2005.05.008. 
70. Inglezakis, V.J.; Poulopoulos, S.G. Chapter 4-Adsorption and Ion-Exchange (Kinetics). In Adsorption, Ion Exchange and Catalysis-Design of Operations and Environmental Applications, 1st ed.; Elsevier Science: Amsterdam, The Netherlands, 2006; pp. 262-266, ISBN-13: 978-0-444-52783-7.

71. Inglezakis V.J. Ion exchange and adsorption fixed bed operations for wastewater treatment Part I: modelling fundamentals and hydraulics analysis. J. Eng. Stud. Res. 2010a, 16(3), 2941, doi:JESR201016V16S01A0004 [0002990].

72. Inglezakis V.J. Ion exchange and adsorption fixed bed operations for wastewater treatment Part II: scale-up and approximate design methods. J. Eng. Stud. Res. 2010b, 16(3), 42-50, doi:JESR201016V16S01A0005 [0002991].

73. International Joint Commission United States and Canada - Office Consolidation. Great Lakes Water Quality Agreement of 1978 - Revised. Available online: www.ijc.org/files/tinymce/uploaded/GLWQA_e.pdf (accessed on 20 April 2018).

74. Jarvie, M.E.; Hand, D.W.; Bhuvendralingam, S.; Crittenden, J.C.; Hokanson, D.R. Simulating the Performance of Fixed Bed Granular Activated Carbon Adsorbers: Removal of Synthetic Organic Chemicals in the Presence of Background Organic Matter. Water Res. 2005, 39(11), 2407-2421, doi:10.1016/j.watres.2005.04.023.

75. Jha, B.; Sigh, D.N. Fly Ash Zeolites, Chapter 2: Basics of Zeolites. Adv. Struct. Mat. 2016, 78, 5-31, doi:10.1007/978-981-10-1404-8_2.

76. Johnson, D. B.; Hallberg, K. B. Acid mine drainage remediation options - a review. Sci. Total Environ. 2005, 338, 3-14, doi:10.1016/j.scitotenv.2004.09.002.

77. Jovanovic, M.; Rajic, N.; Obradovic, B. Novel kinetic model of the removal of divalent heavy metal ions from aqueous solutions by natural clinoptilolite. J. Hazard. Mater. 2012, 233, 57-64, doi:10.1016/j.jhazmat.2012.06.052.

78. Kantarci, N.; Borak, F.; Ulgen, K.O. Bubble column reactors. Process Biochem. 2005, 40, 2263-2283, doi:10.1016/j.procbio.2004.10.004.

79. Kocaoba, S.; Orhan, Y.; Akyuz, T. Kinetics and equilibrium studies of heavy metal ions removal by use of natural zeolite. Desalination 2007, 214, 1-10, doi:10.1016/j.desal.2006.09.023.

80. Kocasoy, G.; Sahin, V. Heavy metal removal from industrial wastewater by clinoptilolite. $J$. Environ. Sci. Heal. A. 2007, 42, 2139-2146, doi:10.1080/10934520701629617.

81. Kostandyan, M.N.; Babayan, S.G.; Balayan, M.A. Effect of heat treatment on the structural characteristics and sorption properties of clinoptilolite. Inorganic Materials. 1982, 18(10), 1498-1501, 0020-1685.

82. Kragovic, M.; Dakovic, A.; Markovic, M.; Krstic, J.; Gatta, G.D.; Rotiroti, N. Characterization of lead sorption by natural and Fe (III)-modified zeolite. Appl. Surf. Sci. 2013, 283, 764-774, doi:10.1016/j.apsusc.2013.07.016.

83. Kuyucak, N.; Akcil, A. Cyanide and removal options from effluents in gold mining and metallurgical processes. Miner. Eng. 2013, 50/51, 13-29, doi:10.1016/j.mineng.2013.05.027. 
84. Largitte, L.; Pasquier, R. A review of the kinetics adsorption models and their application to the adsorption of lead by an activated carbon. Chem. Eng. Res. Des. 2016, 109, 495-504, doi:10.1016/j.cherd.2016.02.006.

85. Lata, S.; Singh, P.K.; Samadder, S.R. Regeneration of adsorbents and recovery of heavy metals: a review. Int. J. Environ. Sci. Technol. 2015, 12, 1461-1478, doi:10.1007/s13762014-0714-9.

86. Langella, A.; Pansini, M.; Cerri, G.; Cappellietti, P.; De Gennaro, M. Thermal Behavior of Natural and Cation-Exchanged Clinoptilolite from Sardinia (Italy). Clay. Clay Miner. 2003, 51(6), 625-633, doi:10.1346/CCMN.2003.0510605.

87. Margeta, K.; Zabukovec Logar, N.; Šiljeg, M.; Farkaš, A. Chapter 5: Natural Zeolites in Water Treatment-How Effective Is Their Use. InTech, Water Treatment, Dr. W. Elshorbagy, Ed., 2013, 81-112, doi:10.5772/50738.

88. Markovic, R.; Gardic, V.; Obradovic, L.; Djordjievski, S.; Stevanovic, Z.; Stevanovic, J.; Gvozdenovic, M. The Application of a Natural Zeolite for Acid Mine Drainage Purification. Mater. Trans., JIM, 2015, 56(12), 2053-2057, doi:10.2320/matertrans.M2015292.

89. Martin, S.; Griswold, W. Human Health Effects of Heavy Metals. Environmental Science and Technology Briefs for Citizens - Center for Hazardous Substance Research. Kansas State University. Issue 15. 2009. Available online: www.engg.ksu.edu/CHSR/ (accessed on 01 September 2014).

90. Masel, R. I. Principles of Adsorption and Reaction on Solid Surfaces. John Wiley \& Sons, Inc. 1996, ISBN: 978-0-471-30392-3. pp. 235-248.

91. McMartin, P. (2014) Mount Polley Mine - Maybe if we tried putting red tape on the breach. Vancouver Sun. Available online: www.ottawacitizen.com/news/metro/Pete+McMartin+Mount+Polley+Mine+Maybe+tried+p utting+tape/10109694/story.html (accessed on 10 August 2014).

92. Minceva, M.; Fajgar, R.; Markovska, L., Meshko, V. Comparative Study of $\mathrm{Zn}^{2+}, \mathrm{Cd}^{2+}$, and $\mathrm{Pb}^{2+}$ Removal From Water Solution Using Natural Clinoptilolitic Zeolite and Commercial Granulated Activated Carbon: Equilibrium of Adsorption. Separ. Sci. Technol. 2008, 43, 2117-2143. doi:10.1080/01496390801941174.

93. Mining Association of Canada (MAC). Mining Facts. Available online: mining.ca/resources/mining-facts (accessed on 01 September 2014).

94. MOECC. Deriving Receiving Water Based Point Source Effluent Requirements for Ontario Waters. 2016. Available online: www.ontario.ca/page/b-1-5-deriving-receiving-waterbased-point-source-effluent-requirements-ontario-waters (accessed 20 April 2018).

95. Mohan, D.; Chander, S. Removal and recovery of metal ions from acid mine drainage using lignite-A low cost sorbent. J. Hazard. Mater. 2006, B137, 1545-1553, doi:10.1016/j.jhazmat.2006.04.053.

96. Motsi, T.; Rowson, N.A.; Simmons, M.J.H. Adsorption of heavy metals from acid mine drainage by natural zeolite. Int. J. Miner. Process. 2009, 92, 42-48, doi:10.1016/j.minpro.2009.02.005. 
97. Motsi, T. Remediation of Acid Mine Drainage using Natural Zeolite. Ph.D. Thesis. School of Chemical Engineering, The University of Birmingham, United Kingdom. 2010.

98. Motsi, T.; Rowson, N.A.; Simmons, M.J.H. Kinetic studies of the removal of heavy metals from acid mine drainage by natural zeolite. Int. J. Miner. Process. 2011, 101, 42-49, doi:10.1016/j.minpro.2011.07.004.

99. Mullin, J. Physical and thermal properties. In Crystallization. Fourth Edition. Read Educational and Professional Publishing Ltd: Woburn, MA, USA, 2001; pp. 76-77, IBSN: 0-7506-4833-3.

100.Mumpton, F.A.; Boles, J.R.; Flanigen, E.M.; Gude, A.J.; Sheppard, R.A.; Hay, R.L.; Surdam, R.C. Chapter 8; Mineralogy and Geology of Natural Zeolites. Washington, D.C.: Mineralogical Society of America. 1977; ISBN 0939950049.

101.Muttil, N.; Chau, K.W. Machine learning paradigms for selecting ecologically significant input variables. Eng. Appl. Artif. Intel. 2007, 20, 735-744, doi:10.1016/j.engappai.2006.11.016.

102. Naja, G.; Volesky, B. Multi-metal biosorption in a fixed-bed flow-through column. Colloid. Surface. 2006, 281, 194-201. doi:10.1016/j.colsurfa.2006.02.040.

103.Nezamzadeh-Ejhieh, A.; Shahanshahi, M. Modification of clinoptilolite nano-particles with hexadecylpyridynium bromide surfactant as an active component of $\mathrm{Cr}(\mathrm{VI})$ selective electrode. J. Ind. Eng. Chem. 2013, 19, 2026-2033, doi:10.1016/j.jiec.2013.03.018.

104. Nezamzadeh-Ejhieh, A.; Shirzadi, A. Enhancement of the photocatalytic activity of Ferrous Oxide by doping onto the nano-clinoptilolite particles towards photodegradation of tetracycline. Chemosphere 2014, 107, 136-144, doi:10.1016/j.chemosphere.2014.02.015.

105. Nicholson, R.V. Acid Drainage and Metal Leaching Prediction: Where have we been and where do we Need to Go - An Historical Perspective. Conference Proc. CIM Symposium, Rouyn-Noranda, QC, Canada, 2015.

106.Nisbet, K.; Rasor, R. An Inventor's Guide to Technology Transfer at the Massachusetts Institute of Technology. MIT Technology Licensing Office. 2005. Available online: web.mit.edu/tlo/www/downloads/pdf/inventors_guide.pdf (accessed on 08 March 2014).

107.Nuic, M.; Trgo, J.; Peric, N.; Vukojevic Medvidovic, N. Analysis of breakthrough curves of $\mathrm{Pb}$ and $\mathrm{Zn}$ sorption from binary solutions on natural clinoptilolite. Micropor. Mesopor. Mat. 2013, 167, 55-61. doi:10.1016/j.micromeso.2012.04.037.

108. Nuic, I.; Trgo, M.; Peric, J.; Vukojevic Medvidovic, N. Uptake of Pb and Zn from a binary solution onto different fixed bed depths of natural zeolite - the BDST model approach. Clay Minerals. 2015, 50, 91-101, doi:10.1180/claymin.2015.050.1.09.

109.Nuic, I.; Trgo, M.; Vukojevic Medvidovic, N. The application of the packed bed reactor theory to $\mathrm{Pb}$ and $\mathrm{Zn}$ uptake from the binary solution onto the fixed bed of natural zeolite. Chem. Eng. J. 2016, 295, 347-357, doi:10.1016/j.cej.2016.03.037.

110. Occupational Health and Safety Act (OHSA). Ontario Regulation 490/09 - Designated Substances. Consolidated Laws - Government of Ontario. Available online: www.elaws.gov.on.ca/html/regs/english/elaws_regs_090490_e.htm (accessed on 01 September 2014). 
111.Olu-Owolabi, B.I.; Diagboya, P.N.; Adebowale, K.O. Evaluation of pyrene sorptionDesorption on tropical soils. J. Environ. Manage. 2014, 137, 1-9, doi:10.1016/j.jenvman.2014.01.048.

112. Ontario Mining \& Exploration Directory \& Resource Guide. Ontario Mining Operations Map. $2015 . \quad$ Available online: www.oma.on.ca/en/resourcesGeneral/Ontario_Mining_Operations_2015.pdf (accessed on 20 April 2018).

113.Oter, O.; Akcay, H. Use of natural clinoptilolite to improve, water quality: Sorption and selectivity studies of lead(II), copper(II), zinc(II), and nickel(II). Water Environ. Res. 2007, 79, 329-335, doi:10.2175/106143006X111880.

114.Othman, M. Z.; Roddick, F. A.; Snow, R. Removal of Dissolved Organic Compounds in Fixed-Bed Columns: Evaluation of Low-Rank Coal Adsorbents. Wat. Res. 2001, 35(12), 2943-2949, doi: S0043-1354(00)00578-9.

115.Ouki, S. K.; Kavannagh, M. Performance of natural zeolites for the treatment of mixed metal contaminated effluents. Waste Manage. Res. 1997, 15 (4), 383-394, doi:10.1006/wmre.1996.0094.

116. Ouki, S.K.; Kavannagh, M. Treatment of metals-contaminated wastewaters by use of natural zeolites. Wat. Sci. Tech. 1999, 39, 115-122, doi:10.1016/S0273-122300260-7.

117.Panayotova, M.; Velikov, B. Kinetics of Heavy Metal Ions Removal by Use of Natural Zeolite. J. Environ. Sci. Health 2002, 37, 139-147, doi:10.1081/E-120002578.

118.Peric, J.; Trgo, M.; Vukojevic Medvidovic, N. Removal of zinc, copper and lead by natural zeolite - a comparison of adsorption isotherms. Water Res. 2004, 38, 1893-1899, doi:10.1016/j.watres.2003.12.035.

119.Peric, J.; Trgo, M.; Vukojevic Medvidovic, N.; Nuic, I. The Effect of Zeolite Fixed Bed Depth on Lead Removal from Aqueous Solutions. Sep. Sci. Technol. 2009, 44, 3113-3127, doi:10.1080/01496390903182230.

120.Perkin Elmer Inc. WinLab32 for ICP-Instrument Control Software, version 5.0; Perkin Elmer Inc.: Waltham MA, USA, 2010.

121.Perkin Elmer Inc. Atomic Spectroscopy-A Guide to Selecting the Appropriate Technique and System: World Leader in AA, ICP-OES, and ICP-MS; Perkin Elmer Inc.: Waltham MA, USA, 2011.

122.Plazinski, W., Rudzinski, W, Plazinska, A. Theoretical models of sorption kinetics including a surface reaction mechanism: A review. Adv. Colloid. Interfac. 2009, 152, 2-13, doi:10.1016/j.cis.2009.07.009.

123.Prasad, B.; Mortimer, R.J.G. Treatment of Acid Mine Drainage Using Fly Ash Zeolite. Water, Air, Soil Pollut. 2011, 218, 667-679, doi:10.1007/s11270-010-0676-6.

124.Qiu, H.; Lv, L.; Pan, B.C.; Zhang, Q.J.; Zhang, W.M.; Zhang, Q.X. Critical review in adsorption kinetic models. J. Zhejiang Univ. Sci. A 2009, 18, 716-724, doi:10.1631/jzus.A0820524. 
125.Rasmussen, E.K. Recent Development - The 1978 Great Lakes Water Quality Agreement and Prospects for U.S.-Canada Pollution Control. Boston College International and Comparative Law Review. 1979, 2(2,11), 499-520.

126.Reed, B. E.; Jamil, M.; Thomas, B. Effect of pH , empty bed contact time and hydraulic loading rate on lead removal by granular activated carbon columns. Water Environ. Res. 1996, 68(5), 877-882, doi:10.2175/106143096X127875.

127.Rice, E.W.; Baird, R.B; Eaton, A.D.; Clesceri, L.S. Part 1000-Introduction, Part 3000METALS. In Standard Methods for the Examination of Water and Wastewater, 22nd ed.; The American Public Health Association (APHA): Washington, DC, USA; The American Water Works Association (AWWA): Denver, CO, USA; The Water Environment Federation (WEF): Alexandria, VA, USA, 2012; pp. 1.1-68, 3.1-112, ISSN 978-087553013-0.

128.Rios, C.A.; Williams, C.D.; Roberts, C.L. Removal of heavy metals from acid mine drainage (AMD) using coal fly ash, natural clinker and synthetic zeolites. J. Hazard. Mater. 2008, 156, 23-35, doi:10.1016/j.jhazmat.2007.11.123.

129. Ritcey, G. M. Tailings management in gold plants. G.M. Ritcey and Associates. Hydrometallurgy, 2005, 78, 3-20, doi:10.1016/j.hydromet.2005.01.001.

130.Riveros, P.; Wong, E.W. Metals Removal from Acid Mine Drainage by Ion Exchange. Report 3.21.1b. Mine Environment Neutral Drainage (MEND) Program. Ontario Ministry of Nerothn Development and Mines, Canada Centre for Mineral and Energy Technology (CANMET), Canada/Northern Ontario Development Agreement (NODA). 1995. Available online: mend-nedem.org/wp-content/uploads/2013/01/3.21.1b.pdf (accessed on 30 October 2014).

131.Salem, A.; Akbari Sene, R. Removal of lead from solution by combination of natural zeolite-kaolin-bentonite as a new low-cost adsorbent. Chem. Eng. J. 2011, 174, 619-628, doi: 10.1016/j.cej.2011.09.075.

132.Singer, P.C.; Stumm, W. Acidic Mine Drainage: The Rate-Limiting Step. Science 1970, 167(3921), 1121-1123.doi:10.1126/science.167.3921.1121.

133.Skoog, D. A.; Holler, F. J.; Crouch, S. R. Principles of Instrumental Analysis. 6th Edition. Thomson - Brooks/Cole. ISBN-13: 978-0-495-01201-6. 2007.

134.Sprynskyy, M.; Buszewski, B; Terzyk, A.P.; Namiesnik, J. Study of the selection mechanism of heavy metal $\left(\mathrm{Pb}^{2+}, \mathrm{Cu}^{2+}, \mathrm{Ni}^{2+}\right.$, and $\left.\mathrm{Cd}^{2+}\right)$ adsorption on clinoptilolite. $J$. Colloid Interface Sci. 2006, 304, 21-28, doi:10.1016/j.jcis.2006.07.068.

135.Stylianou, M.A.; Inglezakis, V.J.; Moustakas, K.G.; Malamis, S.P.; Loizidou, M.D. Removal of $\mathrm{Cu}(\mathrm{II})$ in fixed bed and batch reactors using natural zeolite and exfoliated vermiculite as adsorbents. Desalination, 2007a, 215, 133-142. doi:10.1016/j.desal.2006.10.031.

136.Stylianou, M.A., Hadjiconstantinou, M.P.; Inglezakis, V.J.; Moustakas, K.G.; Loizidou, M.D. Use of natural clinoptilolite for the removal of lead, copper and zinc in fixed bed column. J. Hazard. Mater. 2007b, 143, 575-581. doi:10.1016/j.jhazmat.2006.09.096. 
137.Tchounwou, P.B.; Yedjou, C.G.; Patlolla, A.K.; Sutton, D.J. Heavy Metals Toxicity and the Environment. In: Luch A. (eds) Molecular, Clinical and Environmental Toxicology. Experientia Supplementum, 2012, 101, doi:10.1007/978-3-7643-8340-4_6.

138. Trgo, M.; Peric, J.; Vukojevic Medvidovic, N. A comparative study of ion exchange kinetics in zinc/lead-modified zeolite-clinoptilolite systems. J. Hazard. Mater. 2006, 136, 938-945, doi:10.1016/j.jhazmat.2006.01.032.

139. Tsitsishvili, G.V. Perspectives of Natural Zeolite Applications. Occurrence. In Properties and Utilization of Natural Zeolites-2nd International Conference 1985; Akademiai Kiado: Budapest, Hungary, 1988; pp. 367-393.

140.University of Liverpool. Zeolite - Clinoptilolite. Nick Greeves, ChemTube3D, 2017. Available online: www.chemtube3d.com/solidstate/SS-Z-Clinoptilolite.htm (accessed on 20 April 2018).

141.University of Michigan (LSA). ICP-OES User's Booklet. Available online: lsa.umich.edu/content/dam/chem-assets/chem-docs/ICP_OES_User_s_Booklet.doc (accessed on 06 July 6 2015).

142.US EPA Guidance on Choosing a Sampling Design for Environmental Data Collection Use in Developing a Quality Assurance Project Plan. EPA QA/G-5S. Office of Environmental Information, Washing, DC 20460. 2002.

143. Van Reeuwijk, L.P. The Thermal Dehydration of Natural Zeolites. Department of Soil Science and Geology, Agricultural University, Mededelingen Landbouwhogeschool Wageningen 74-9, The Netherlands, 1974.

144.Vaca-Mier, M.; Lopez-Callejas, R.; Gehr, R.; Jimenez-Cisneros, B. E; Alvarez, P. J. J. Heavy metal removal with Mexican Clinoptilolite: Multi-Component Ionic Exchange. Wat. Res. 2001, 35(2), 373-378, doi:10.1016/S0043-1354(00)00270-0.

145. Vukojevic Medvidovic, N.; Peric, J.; Trgo, M. Column performance in lead removal from aqueous solutions by fixed bed of natural zeolite-clinoptilolite. Sep. Purif. Technol. 2006, 49, 237-244, doi:10.1016/j.seppur.2005.10.005.

146. Vukojevic Medvidovic, N.; Peric, J.; Trgo, M.; Nuic, I.; Ugrina, M. Design of Fixed Bed Column for Lead Removal on Natural Zeolite Based on Batch Studies. Chem. Biochem. Eng. Q. 2013, 27, 21-28, doi:http://hrcak.srce.hr/99433.

147.Wang, C.; Li, J.; Sun, X.; Wang, L.; Sun, X. Evaluation of zeolites synthesized from fly ash as potential adsorbents for wastewater containing heavy metals. J. Environ. Sci. 2009, 21, 127-136, doi:10.1016/S1001-074260022-X.

148.Wang, S.; Peng, Y. Natural zeolites as effective adsorbents in water and wastewater treatment. Chem. Eng. J. 2010, 156, 11-24, doi:10.1016/j.cej.2009.10.029.

149.Wang, W.C.; Xu, D.M.; Chau, K.W.; Lei, G.J. Assessment of river water quality based on theory of variable fuzzy sets and fuzzy binary comparison method. Water Resour. Manag. 2014, 28, 4183-4200, doi:10.1007/s11269-014-0738-4. 
150.Wilson, L.J. Canada-Wide Survey of Acid Mine Drainage Characteristics. Project Report 3.22.1-Job No. 50788. Mineral Sciences Laboratories Division Report MSL 94-32 (CR). Ontario Ministry of Northern Development and Mines. Mine Environment Neutral Drainage (MEND) Program: Canada, 1994. Available online: http://mend-nedem.org/wpcontent/uploads/2013/01/3.22.1.pdf (accessed on 30 October 2014).

151.Wingenfelder, U.; Hansen, C.; Furrer, G.; Schulin, R. Removal of Heavy Metals from Mine Waters from Natural Zeolites. Environ. Sci. Technol. 2005, 39, 4606-4613, doi:10.1021/es048482s.

152.Wu, F.C.; Tseng, R.L.; Juang, R.S. Characteristics of Elovich equation used for the analysis of adsorption kinetics in dye-chitosan systems. Chem. Eng. J. 2009, 150, 366-373, doi:10.1016/j.cej.2009.01.014.

153.Xu, W.; Li, L.Y.; Grace, J.R. Zinc removal from acid rock drainage by clinoptilolite in a slurry bubble column. Appl. Clay Sci. 2010, doi:10.1016/j.clay.2010.07.005.

154. Xu, W.; Li, L.Y.; Grace, J.R. Regeneration of natural Bear River clinoptilolite sorbents used to remove $\mathrm{Zn}$ from acid mine drainage in a slurry bubble column. Appl. Clay Sci. 2012, 55, 83-87, doi:10.1016/j.clay.2011.10.009.

155.Xu, W.; Li, L.Y.; Grace, J.R., Hebrard, G. Acid rock drainage treatment by clinoptilolite with slurry bubble column: Sustainable zinc removal with regeneration of clinoptilolite. Appl. Clay Sci. 2013, 80-81, 31-37, doi:10.1016/j.clay.2013.05.009.

156.Xu, W.; Li, L.Y.; Grace, J.R. Dealumination of clinoptilolite and its effect on zinc removal from acid rock drainage. Chemosphere 2014, 111, 427-433, doi:10.1016/j.chemosphere.2014.03.125.

157. Yabe, M.J.S.; de Oliveira, E. Heavy metals removal in industrial effluents by sequential adsorbent treatment. Adv. Environ. Res. 2003, 7, 263-272, doi:S1093-0191(01)00128-9.

158. Yörükoğulları, E.; Yılmaz, G.; Dikmen, S. Thermal treatment of zeolitic tuff. J. Therm. Anal. Calorim. 2010, 100(3), 925-928, doi:10.1007/s10973-009-0503-8.

159.Zhao, M.Y.; Cheng, C.T.; Chau, K.W.; Li, G. Multiple criteria data envelopment analysis for full ranking units associated to environment impact assessment. Int. J. Environ. Pollut. 2006, 28, 448-464, doi:10.1504/IJEP.2006.011222.

160.Zinck, J. Review of Disposal, Reprocessing and Reuse Options for Acidic Drainage Treatment Sludge. Report 3.42.3. Mine Environment Neutral Drainage (MEND) Program. Mining Association of Canada. CANMET Mining and Mineral Sciences Laboratories. Canada, 2005. Available online: mend-nedem.org/wp-content/uploads/2013/01/3.42.3.pdf (accessed on 30 October 2014).

161.Zolgharnein, J.; Shahmoradi, A. Characterization of Sorption Isotherms, Kinetic Models, and Multivariate Approach for Optimization of $\mathrm{Hg}(\mathrm{II})$ Adsorption onto Fraxinus Tree Leaves. J. Chem. Eng. Data 2010, 55, 5040-5049, doi:10.1021/je1006218. 